\title{
Development of METx: a Vasopressin Agonist
}

\author{
Sunish Patel
}

A dissertation submitted in partial fulfilment of the requirements for the degree of

Doctor of Philosophy

at the

School of Pharmacy,

University College London 


\section{Declaration}

This thesis describes research conducted at the UCL School of Pharmacy between 12th November 2012 and 20th January 2016 under the supervision of Professor Ijeoma Uchegbu. I certify that the research described is original and that any parts of the work that have been conducted by collaboration are clearly indicated. I also certify that I have written all the text herein and have clearly indicated by suitable citation any part of this dissertation that has already appeared in publication.

Sunish Patel:

Date: 


\section{Abstract}

Vasopressin (AVP) was one of the first peptides to be extracted and produced synthetically in the early 1950s. Since then AVP and its analogues have been increasingly researched as both AVP agonists and antagonists have therapeutic uses. Although much time and effort has been used to design potent selective AVP agonists and antagonists, very few have reached the market. AVP agonist analogues have only been approved for diabetes insipidus and variceal bleeding. Current main stay treatment for diabetes insipidus is desmopressin, a synthetic analogue of AVP. Desmopressin is well tolerated by the majority of patients, however severe adverse affects, such as hyponatraemia and water intoxication require a strict fluid intake, restricting fluid intake. Our aim is to develop METx for the treatment of diabetes insipidus to help overcome some of the limitations in lifestyle. The solubility and aggregation of METx was measured using the shake flask and pyrene assay respectively. The activity of METx was determined by measuring cAMP production by MDCK cells. The activity of METx was compared to that AVP, the natural agonist of the vasopressin receptor. Formulation studies were performed using GCPQ to create a formulation that could be used subcutaneously or orally. The stability of GCPQ formulations was determined in plasma, simulated gastric fluid (SGF) and rat intestinal wash (RIW). The activity of METx was measured in vivo by monitoring the urine production of rats upon administration of METx intravenously. METx was practically insoluble in aqueous solvents such as water and PBS, with increased solubility in organic solvents. Using TEM imaging aggregation of METx of was seen, the pyrene assay determined the critical micelle concentration to be $27.4 \mathrm{mg}^{-1}$. METx was found to be a partial agonist of the vasopressin 2 receptor naturally expressed on the MDCK cells, it exhibited a similar $\mathrm{EC}_{50}$ to AVP. Formulating with GCPQ a ratio of 1:5 (METx:GCPQ) was found to be the most stable in plasma, SGF and RIW. In vivo studies found METx to have a dose dependent reduction in urine production in rats, with $40 \mathrm{mg} \mathrm{kg}^{-1}$ rats not producing any urine. The experiments suggest the development of a novel partial agonist of the vasopressin 2 receptor in vitro. The addition of GCPQ results in the formation of nanoparticles, with a ratio of 1:5 (METx:GCPQ) most stable in plasma, SGF and RIW. In vivo experiments resulted in reduced urine production by rats, in a dose responsive manner. 


\section{Acknowledgements}

I would give my deepest gratitude to my primary supervisor Prof. Ijeoma Uchegbu for giving me the opportunity to do this $\mathrm{PhD}$, and for her guidance throughout the project. I also would like to give a special thanks to my secondary supervisor Prof. Andreas Schatzlein for all his all of his helpful guidance and support. I would also like to thank both of supervisors for all the added opportunities they gave me within the lab.

This thesis would have not be possible without all the support of the various members of the lab. I am grateful to all the members within lab 326 and 105, especially those who discussed my experiments and results with, and listened to me moan and groan. A special thank you to Dr. Antonio Iannitelli for all the help he gave me everyday, from the first day till the very end. A second special thanks to Dr. Lisa Godfrey, for without you all the animal experiments would not have been possible, I specially want to thank you for your patience, you did not let me faint. I am grateful to Dr. Lorenzo Capretto, Xian Weng, Nick Hobson and Dr. David Workman for their encouragement and friendship, I will miss the coffee times. Each of you helped to make the lab a great place to be, you each gave the lab and the office that special buzz.

A special thank you to my parents and brother, who have always pushed me to climb the next mountain. I could never thank you enough for being there everyday, every step I take and making my life so blessed. Also my life would not be the same without my mandir family, my second family always there to show me the stars in the dark sky, trying to make me be positive in every situation. My journey would not be the same without all my friends, but a special thanks has to go to Amsher, Brijesh, Minal, Rehan and Arunan. There is no way I could even describe what each of you has done for me, how thankful and lucky I am to have such amazing friends and family, each of you are one in a million. Thank you for all your motivation, encouragement, unconditional love, support and patience.

Finally, thank you to everyone else around me, not just for these 4 years, but thank you for everything from the day that this journey began, in the late 80s. I would not have got here without any of you! 


\section{Contents}

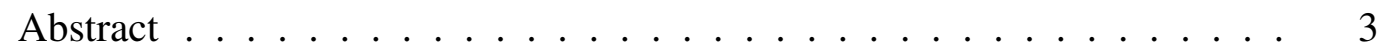

Acknowledgments . . . . . . . . . . . . . . . . . 4

List of Figures . . . . . . . . . . . . . . . . . . . . . 9

List of Tables . . . . . . . . . . . . . . . . . . 9

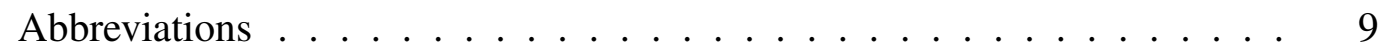

$\begin{array}{lll}1 & \text { Introduction } & 20\end{array}$

1.1 Peptide Therapeutics $\ldots \ldots \ldots \ldots \ldots$

1.1 .1 Vasopressin and Oxytocin $\ldots \ldots \ldots \ldots \ldots . \ldots 22$

1.2 Peptide Drug Delivery $\ldots \ldots \ldots$

$1.2 .1 \quad$ Oral Delivery of Peptides $\ldots \ldots \ldots \ldots$

1.2 .2 Subcutaneous Delivery $\ldots \ldots \ldots \ldots \ldots$

1.3 Methods to Overcome Factors for Peptide Delivery . . . . . . . . . 39

$1.3 .1 \quad$ Chemical Modification . . . . . . . . . . . . . . . . . . . 39

1.3 .2 Protease Inhibitors $\ldots \ldots \ldots \ldots$

1.3 .3 Absorption Enhancers $\ldots \ldots \ldots \ldots \ldots$ 
$1.3 .4 \quad$ Particulate Carrier Delivery Systems . . . . . . . . . . . . 47

1.4 Aims and Objectives $\ldots \ldots \ldots . \ldots . \ldots 53$

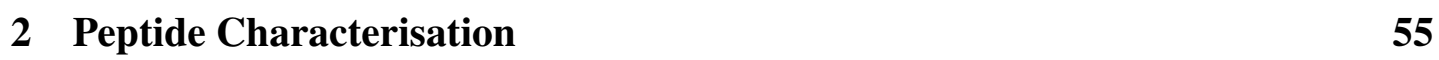

2.1 Introduction . . . . . . . . . . . . . . . . . 55

2.1 .1 Mass Spectrometry . . . . . . . . . . . . . 55

2.1 .2 Transmission electron microscopy (TEM) . . . . . . . . . 57

$2.1 .3 \quad$ Critical Micelle Concentration . . . . . . . . . . . . . 58

2.1 .4 HPLC . . . . . . . . . . . . . . . . . 59

2.1 .5 Solubility . . . . . . . . . . . . . . 60

2.2 Materials \& Method $\ldots \ldots \ldots$. . . . . . . . . . . . . . . . . 62

$2.2 .1 \quad$ Materials . . . . . . . . . . . . . . . . 62

2.2 .2 Methods . . . . . . . . . . . . . . 63

2.3 Results . . . . . . . . . . . . . . . . . . . . . 66

$2.3 .1 \quad$ Mass Spectrometry $\ldots \ldots \ldots 6$

2.3 .2 TEM $\ldots \ldots \ldots \ldots \ldots$

$2.3 .3 \quad$ Pyrene Assay . . . . . . . . . . . . . . . . . . . . . . 69

2.3 .4 HPLC . . . . . . . . . . . . . . . . . . . 70

2.3 .5 Solubility Studies . . . . . . . . . . . . . . 71

2.4 Discussion . . . . . . . . . . . . . . . . . . . . . . . . 73

\begin{tabular}{|ll|}
\hline 3 & In Vitro Studies \\
\hline
\end{tabular} 
3.1 Introduction . . . . . . . . . . . . . . . . . 76

$3.1 .1 \quad$ Vasopressin . . . . . . . . . . . . . . . 76

3.1 .2 Ligand Screening Studies . . . . . . . . . . . . 78

3.1 .3 Enzyme Immunoassay . . . . . . . . . . . . . 83

3.2 Materials \& Methods . . . . . . . . . . . . . . . . . . . . . . . 87

$3.2 .1 \quad$ Materials . . . . . . . . . . . . . . . . 87

3.2 .2 Methods . . . . . . . . . . . . . . . . . . . . . . . . 88

3.3 Results . . . . . . . . . . . . . . . . . . . . . . . . 91

3.3.1 AVP and METx Dose Response Curves . . . . . . . . . . . 91

3.3.2 AVP and METx Competition Experiments . . . . . . . . . . 97

$3.3 .3 \quad$ Synergistic Studies . . . . . . . . . . . . . . . . . 101

3.4 Discussion . . . . . . . . . . . . . . . . . . . . 103

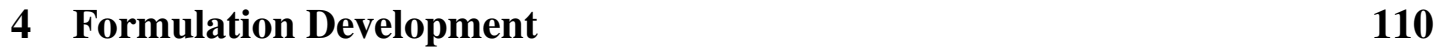

$4.1 \quad$ Introduction $\ldots \ldots \ldots \ldots$. . . . . . . . . . . . . . . . 110

$4.1 .1 \quad$ Polymer Characterisation Methods . . . . . . . . . . . . . . . 111

4.1 .2 Formulation Characterisation Methods. . . . . . . . . . . . . 114

4.2 Materials \& Methods . . . . . . . . . . . . . . . . . . . . . 116

$4.2 .1 \quad$ Materials . . . . . . . . . . . . . . 116

4.2 .2 Methods . . . . . . . . . . . . . . . . . 118

4.3 Results . . . . . . . . . . . . . . . . . . . . . . . . 130

$4.3 .1 \quad$ Polymer Synthesis $\ldots \ldots$. . . . . . . . . . . . . . 130 
4.3 .2 Formulation Development . . . . . . . . . . . . . . 137

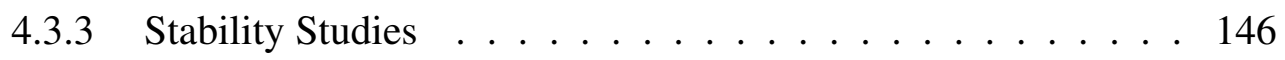

4.4 Discussion $\ldots \ldots \ldots \ldots \ldots$

$\begin{array}{lll}5 & \text { In Vivo Studies } & 162\end{array}$

5.1 Introduction . . . . . . . . . . . . . . . . . . 162

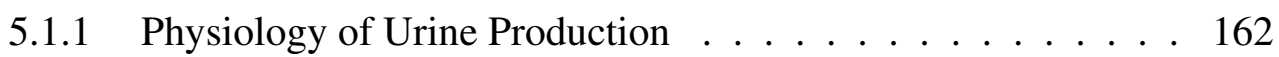

5.1 .2 Urine Disorders . . . . . . . . . . . . . . . . . . . . . 164

5.2 Materials Methods . . . . . . . . . . . . . . . . . 167

$5.2 .1 \quad$ Materials . . . . . . . . . . . . . . . 167

5.2 .2 Methods . . . . . . . . . . . . . 167

5.3 Results . . . . . . . . . . . . . . . . . . . . 170

$5.3 .1 \quad$ Intravenous Study . . . . . . . . . . . . . 170

5.3 .2 Oral Study . . . . . . . . . . . . . . . . . . . 172

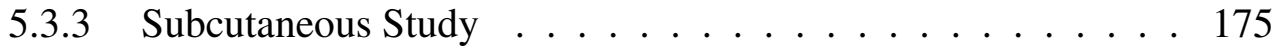

5.4 Discussion . . . . . . . . . . . . . . . . . . . . . . . 179

6 Conclusions \& Future Work 182

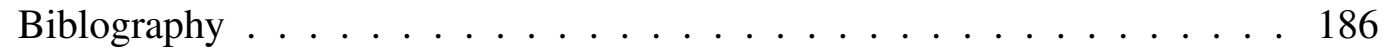




\section{List of Figures}

$1.1 \quad$ Structure and sequence of AVP and OXT, difference in sequence and structure are highlighted in blue . . . . . . . . . . . 23

1.2 Structure activity relationship summary of AVP activity at $\mathrm{V}_{2} \mathrm{R}$. . . 26

1.3 Oral peptide technologies in different stages of clinical trials [145],

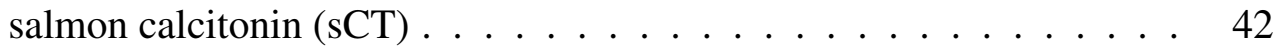

1.4 Structure of METx; red shows modification $\ldots \ldots \ldots 3$

2.1 Tandem MSMS of METx; A: Structure of METx with fragmentation sites, B: MSMS of METx with fragmentation peaks. . . . . . . . . 67

2.2 Tandem MSMS of METx; A: Structure of METx with fragmentation sites, B: MSMS of METx with fragmentation peaks. . . . . . . . . . 68

2.3 TEM pictures of METx suspended (A and $\mathrm{B}$ ) and sonication ( $\mathrm{C}$ and D) in water at $1 \mathrm{mg} \mathrm{ml}^{-1} \ldots \ldots \ldots$. . . . . . . . . . . 69

2.4 Determination of CMC of METx using pyrene assay. . . . . . . . 70

2.5 HPLC calibration curve of METx. . . . . . . . . . . . . 71

$3.1 \mathrm{~V}_{2} \mathrm{R}$ cellular cascade. $\ldots \ldots \ldots \ldots \ldots \ldots \ldots \ldots$

3.2 Ligand binding saturation curve. . . . . . . . . . . . . . 82

3.3 Overview of different EIA methods. . . . . . . . . . . . . 86 
3.4 Dose response curve of AVP and METx in MDCK cells in 96 well plates. 91

3.5 Dose response curve of AVP and METx in MDCK cells in 12 well transwell plates. . . . . . . . . . . . . . . . . 93

3.6 Dose response curve of AVP and METx in MDCK cells in 24 well plates. 95

3.7 Competition experiment of AVP and METx in 96 well plates. . . . . . 97

$3.8 \quad$ Competition experiment of AVP and METx in transwell. . . . . . . . 98

3.9 Competition experiment of AVP and METx in 24 well plate. . . . . . 100

3.10 Synergistic effects of AVP and METx in 96 well plate. . . . . . . . . 101

3.11 Receptor reserve of 24,96 and transwell plates. . . . . . . . . . 106

$4.1 \quad$ Flow diagram of GCPQ synthesis. . . . . . . . . . . . . . . 118

4.2 Reaction mechanism of palmitoyaltion. . . . . . . . . . . . . . 119

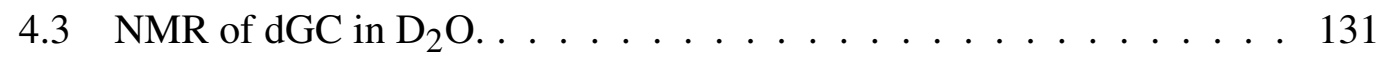

4.4 NMR of PGC in mixture of $\mathrm{MeO}-\mathrm{d}_{4}, \mathrm{D}_{2} \mathrm{O}$ and AcOD (40:20:5). . . . 133

4.5 NMR of GCPQ in $\mathrm{CD}_{3} \mathrm{OD} \mathrm{DCl}(70: 1) \ldots \ldots \ldots$

4.6 Negatively stained TEM images of METx:GCPQ (1:4), white arrows point to fibrous structures, (GCPQ batch: 051212 METx concentration $\left.1 \mathrm{mg} \mathrm{ml}^{-1}\right) . \ldots \ldots \ldots \ldots \ldots \ldots$

4.7 Negatively stained TEM images of METx:GCPQ (1:5), white arrows point to fibrous structures, (GCPQ batch: 051212. METx concentration $\left.1 \mathrm{mg} \mathrm{ml}^{-1}\right) . \ldots \ldots \ldots \ldots \ldots$

4.8 Negatively stained TEM images of METx:GCPQ (1:10) (GCPQ batch:

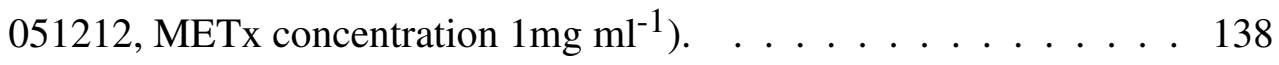


4.9 DLS intensity distribution graph of METx:GCPQ formulations, (GCPQ batch: 051212, METx concentration $\left.1 \mathrm{mg} \mathrm{ml}^{-1}\right)$. . . . . . . . . . 139

4.10 Negatively stained TEM images of METx:GCPQ (Glycerol) (1:1), white arrows show presence of fibrous material, (GCPQ batch: 210115, METx concentration $\left.1 \mathrm{mg} \mathrm{ml}^{-1}\right) . \ldots \ldots \ldots . \ldots \ldots 14$

4.11 Negatively stained TEM images of METx:GCPQ (Glycerol) (1:5), white arrows show presence of fibrous material, (GCPQ batch: 210115, METx concentration $\left.1 \mathrm{mg} \mathrm{ml}^{-1}\right)$. . . . . . . . . . . . . . 141

4.12 Negatively stained TEM images of METx:GCPQ (Glycerol) (1:10), white arrows show presence of fibrous material, (GCPQ batch: 210115, METx concentration $\left.1 \mathrm{mg} \mathrm{ml}^{-1}\right) . \ldots \ldots \ldots$. . . . . . . 141

4.13 r METx:GCPQ (glycerol) formulations, (GCPQ batch: 210115, METx concentration $\left.1 \mathrm{mg} \mathrm{ml}^{-1}\right) . \ldots \ldots \ldots \ldots$. . . . . . . . 142

4.14 METx:GCPQ (glycerol) formulations prepared with either GCPQ, AcOH or glycerol omitted. . . . . . . . . . . . . . . . . . . 145

4.15 Stability of METx:GCPQ formulations (1:4), (1:5) and (1:7) in rat plasma (30\%), (final METx concentration 100 $\mathrm{mcg} \mathrm{ml}^{-1}$, GCPQ:051212).

*= significant difference vs METx, \#= significant difference vs METx:GCPQ (1:4), "=significant difference vs METx:GCPQ (1:7); p<0.05. . . . . . 146

4.16 Stability of METx:GCPQ (glycerol) formulations (1:1), (1:5) and (1:10) in rat plasma (30\%), (final concentration of METx 100 $\mathrm{mcg} \mathrm{ml}^{-1}$, GCPQ:1506 15).

${ }^{*}=$ significant difference vs METx,\#= significant difference vs METx:GCPQ (1:1), "=significant difference vs METx:GCPQ (1:10); p<0.05. . . . . 147

4.17 Stability of METx:GCPQ (glycerol) formulations (1:1), (1:5) and (1:10) in rat plasma (10\%) (final concentration of METx 100 $\left.\mathrm{mcg} \mathrm{m}^{-1}, \mathrm{GCPQ}: 150615\right)$. *=significant difference vs METx,\#= significant difference vs METx:GCPQ (1:1), "=significant difference vs METx:GCPQ (1:10); p<0.05. . . . . 148 
4.18 Stability of METx:GCPQ formulations (1:4), (1:5) and (1:10) in SGF (final concentration of METx 25mcg ml${ }^{-1}$, GCPQ:051212).*= significant difference vs METx,\#= significant difference vs METx:GCPQ $(1: 10) ; \mathrm{p}<0.05 . \ldots \ldots \ldots \ldots \ldots$

4.19 Stability of METx:GCPQ formulations (1:5) and (1:10) in RIW (final concentration of METx $25 \mathrm{mcg} \mathrm{ml}^{-1}$ GCPQ:051212), *= significant difference vs METx,\#= significant difference vs METx:GCPQ $(1: 100) ; \mathrm{p}<0.05 \ldots \ldots \ldots \ldots \ldots \ldots \ldots$

4.20 Peptide content of METx:GCPQ (glycerol) (1:5) stored at room temperature and at $4{ }^{\circ} \mathrm{C}$ (GCPQ:050815), *= significant difference vs day $0 ; \mathrm{p}<0.05$.

4.21 Nanoparticle size of METx:GCPQ (glycerol) (1:5) stored at room temperature and at $4^{\circ} \mathrm{C},(\mathrm{GCPQ}: 050815)$.

5.1 Concentrating of Urine in Nephrons of the kidney. . . . . . . . . . . . 163

5.2 Effect of intravenous administration METx:GCPQ on urine volume of male SD rats over 4 hours $(\mathrm{n}=3),(\mathrm{GCPQ}: 051212)$; *=significant difference to control $\mathrm{p}<0.05 . \ldots \ldots \ldots$. . . . . . . . 170

5.3 Effect of intravenous administration METx:GCPQ on urine osmolality of male SD rats over 4 hours $(n=3) ; *^{*}=$ significant difference to un-

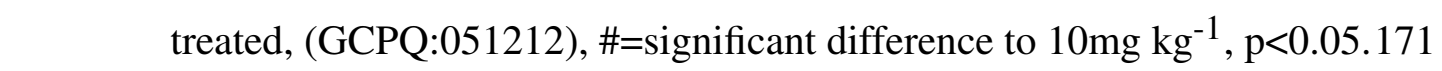

5.4 Effect of oral administration METx:GCPQ (1:4) $\left(100 \mathrm{mg} \mathrm{kg}^{-1}\right)$ on urine volume of male SD rats over 4 hours $(\mathrm{n}=5),(\mathrm{GCPQ}: 121114)$. . . . 172

5.5 Effect of oral administration METx:GCPQ (1:4) $\left(200 \mathrm{mg} \mathrm{kg}^{-1}\right.$ on urine volume of male SD (n=5) rats over 4 hours, (GCPQ:121114). . . . . . 173

5.6 Effect of oral administration METx:GCPQ $\left(100 \mathrm{mg} \mathrm{kg}^{-1}\right)$ on urine osmolality of urine produced by male SD rats over 4 hours (n=5), (GCPQ:121114). 174 
5.7 Effect of oral administration METx:GCPQ $\left(200 \mathrm{mg} \mathrm{kg}^{-1}\right)$ on urine osmolality of urine produced by male SD rats over 4 hours $(n=5),($ GCPQ: 121114). . . . . . . . . . . . . . . . . 174

5.8 Effect of subcutaneous administration METx:GCPQ (1:5) on urine volume production by male SD rats $(\mathrm{n}=5)$ over 4 hours, (GCPQ:210115). 175

5.9 Effect of subcutaneous administration METx:GCPQ (glycerol) (1:5) on urine volume production by male SD rats $(\mathrm{n}=5)$ over 4 hours, (GCPQ:090315). 176

5.10 Effect of subcutaneous administration METx:GCPQ (1:5) on urine osmolality of urine produced by male SD rats $(n=5)$ over 4 hours, (GCPQ:210115). . . . . . . . . . . . . . . 177

5.11 Effect of subcutaneous administration METx:GCPQ (glycerol) (1:5) on urine osmolality of urine produced by male SD rats $(\mathrm{n}=5)$ over 4 hours, (GCPQ:090315). . . . . . . . . . . . . . . . . 177 


\section{List of Tables}

1.1 Advantages and disadvantages of peptide based therapeutic agents [3,

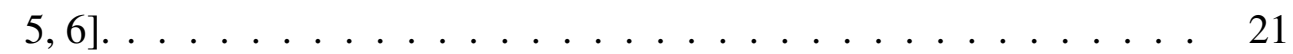

1.2 Binding affinity of AVP and OXT for human AVP and OXT receptors

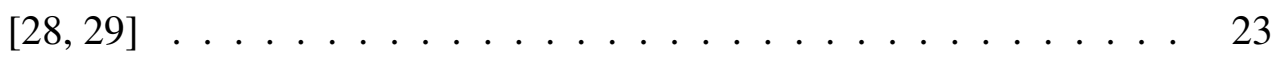

1.3 Route of peptide drug administration: advantages and disadvantages [62, 63].

$2.1 \quad$ Definition of solubility from BP 2016 [245]. . . . . . . . . . . . . . 60

$2.2 \quad$ Materials used for physiochemical studies of METx $\ldots \ldots$. . . . . . 62

2.3 HPLC solvent gradient for METx. . . . . . . . . . . . . . . . 64

2.4 Identified MSMS fragments of METx. . . . . . . . . . . . . . 66

$2.5 \quad$ Solubility of METx in different solvents. . . . . . . . . . . . . 71

3.1 Commercial availability, advantages and disadvantages of different GCPR screening techniques $[276,278]$. . . . . . . . . . . . . 79

$3.2 \quad$ Materials used for in vitro studies of METx. . . . . . . . . . . . . . . 87

$3.3 \quad \mathrm{EC}_{50}$ of AVP and METx in different well plate. . . . . . . . . . 96

$4.1 \quad$ Materials used for formulation development of METx. . . . . . . . 116 
4.3 Chemical shift assignment of dGC NMR. . . . . . . . . . . . . 130

4.4 Chemical shift assignment of PGC NMR. . . . . . . . . . . . . . . 132

4.5 Chemical shift assignment of GCPQ NMR. . . . . . . . . . . . . . . 134

4.6 Molecular weights of GCPQ measured by GPC. . . . . . . . . . . . . 136

4.7 DLS Peak Size and Population of METx:GCPQ formulations, (GCPQ batch: 051212, METx concentration $\left.1 \mathrm{mg} \mathrm{ml}^{-1}\right)$.

4.8 DLS Z-average, PDI and volume mean sizes of METx:GCPQ formulations, (GCPQ batch: 051212, METx concentration $\left.1 \mathrm{mg} \mathrm{m}^{-1}\right)$.

4.9 DLS Peak Sizes and Population Percent of METx:GCPQ (glycerol) formulations, (GCPQ batch: 210115, METx concentration $1 \mathrm{mg} \mathrm{ml}^{-1}$ ). 142

4.10 DLS Z-average and PDI of METx:GCPQ (glycerol) formulations, (GCPQ batch: 210115, METx concentration $\left.1 \mathrm{mg} \mathrm{ml}^{-1}\right)$. . . . . . . . . . . 143

4.11 Results of role of glycerol study. . . . . . . . . . . . . . . . . . . 143

5.1 Common causes of polyuria [402] $\ldots \ldots \ldots$. . . . . . . . . . . 164

$5.2 \quad$ Materials used for in vivo studies of METx. . . . . . . . . . . . . . 167 


\section{Abbreviations}

\begin{tabular}{|c|c|}
\hline Abbreviation & Defintion \\
\hline${ }^{1} \mathrm{H}$ NMR & Proton NMR \\
\hline${ }^{13} \mathrm{C} \mathrm{NMR}$ & Carbon NMR \\
\hline $\mathrm{AcOH}$ & Acetic Acid \\
\hline ACTH & Adrenocorticotropin \\
\hline AcOH-d6 & Deuterated Acetic Acid \\
\hline $\mathrm{ADH}$ & Anti Diuretic Hormone \\
\hline AFM & Atomic Force Microscopy \\
\hline AQP1 & Aquaporin 1 Water Channels \\
\hline AQP2 & Aquaporin 2 Water Channels \\
\hline ATP & Adenosine Triphosphate \\
\hline AVP & Arginine Vasopressin \\
\hline AVP & Arginine Vasotocin \\
\hline $\mathrm{BP}$ & British Pharmacopeia \\
\hline BMI & Body Mass Index \\
\hline BSA & Bovine Serum Albumin \\
\hline cAMP & Cyclic Adenosine Monophosphate \\
\hline CDI & Central Diabetes Insipidus \\
\hline $\mathrm{CHO}$ & Chinese Hamster Ovary \\
\hline $\mathrm{CMC}$ & Critical Micelle Concentration \\
\hline $\mathrm{D}_{2} \mathrm{O}$ & Deutrated Water \\
\hline DAG & Diacylglycerol \\
\hline $\mathrm{dGC}$ & Degraded Glycol Chitosan \\
\hline DI & Diabetes Insipidus \\
\hline KA DLS & Dynamic Light Scattering \\
\hline $\mathrm{DMF}$ & Dimethylformaide \\
\hline DPBS & Dulbecco's Phosphate-Buffered Saline \\
\hline DMSO & Dimethyl Sulfoxide \\
\hline EACC & European Collection of Authenticated Cell Cultures \\
\hline $\mathrm{ECM}$ & Extra Cellular Matrix \\
\hline
\end{tabular}


ELISA

EMA

EMEM

ESI

$\mathrm{EtOH}$

Et2O

$\mathrm{Et}_{3} \mathrm{~N}$

EUP

FBS

FCS

FDA

GAG

GC

GCPQ

GI

GLP

GPC

GPC-MALLS

GPCR

HA

$\mathrm{HCl}$

$\mathrm{HCP}$

HEK

HPLC

IBMX

IFN

IL-7

IP

$\mathrm{IP}_{3}$

IUPAC

IV
Enzyme-Linked Immunosorbent Assay

European Medicines Agency

Eagle's Minimum Essential Medium

Electro Spray Ionisation

Absoulte Ethanol

Ether

Triethylamine

European Pharmacopeia

Fetal Bovine Serum

Fluorescence Correlation Spectroscopy

US Food and Drug Administration

Glycosaminoglycan

Glycol Chitosan

Quaternary Ammonium Palmitoyl Glycol Chitosan

Gastro-Intestinal

Glucagon like peptide

Gel Permeation Chromatography GPC Multi Angle Laser Light Scattering

G Protein Coupled Receptors

Hyaluronic Acid

Hydrochloric Acid

Health Care Professional

Human Embryonic Kidney

High performance Liquid Chromatography

Isobutylmethylxanthine

Interferon

Interleukin 7

Intraperitoneal

Inositol Trisphosphate

International Union of Pure and Applied Chemistry

Intravenous 
MALDI

$\mathrm{MCN}$

MDCK

$\mathrm{MeCN}$

$\mathrm{Me}_{3} \mathrm{I}$

$\mathrm{MeOH}$

$\mathrm{MeOH}-\mathrm{d} 4$

MPS

MS

MSMS

MWCO

$\mathrm{m} / \mathrm{z}$

$\mathrm{NaCl}$

$\mathrm{NaI}$

$\mathrm{NaOH}$

NDI

$\mathrm{NE}$

NEAA

NMP

NMR

NTA

OXT

OXTR

PBS

PCL

PDI

PKA

PLA

PLGA

PNS
Matrix Assisted Laser Desorption/Ionisation

Magnocellular Neurons

Madin-Darby Canine Kidney Acetonitrile Methyl iodide Methanol Deuterated Methanol Monophagous system

Mass Spectrometry Tandem Mass Spectrometry Molecular Weight Cut Off Mass to Charge ratio Sodium Chloride Sodium Iodidie Sodium Hydroxide Nephrogenic Diabetes Insipidus Nocturnal Enuresis Non Essential Amino Acid N-Methyl-2-pyrrolidone Nuclear Magnetic Resonance Nanoparticle Tracking Analysis Oxytocin Oxytocin Receptor Phosphate Buffered Saline Polycaprolactone Polydispersity Index Protein Kinase A Poly Lactic Acid Poly(lactic-co-glycolic acid) Palmitic acid N-hydroxysuccinimide 
PGC

QELS

$\mathrm{rHu}$

RIW

RT

$\mathrm{SC}$

$\mathrm{sCT}$

SGF

TAL

TDL

TEM

TMC

TMS

USP

$\mathrm{V}_{1 \mathrm{~A}} \mathrm{R}$

$\mathrm{V}_{1 \mathrm{~B}} \mathrm{R}$

$\mathrm{V}_{2} \mathrm{R}$

WHO
Palmitoyl Glycol Chitosan

Quasi Ecstatic Light scattering

Recombinant Human Hyaluronidase

Rat intestinal wash

Room Temperature subcutaneous

Salmon Calcitonin

$\mathrm{S}$ imulated gastric fluid

Thick Ascending Limb

Thin Descending Limb

Transmission Electro Microscopy

Trimethyl Chitosan

Tetramethylsilane

US Pharmacopeia

Vasopressin 1A receptor

Vasopressin 1B receptor

Vasopressin 2 receptor

World Health Organisation 


\section{Chapter 1: Introduction}

\subsection{Peptide Therapeutics}

Peptides are defined as a short chain of amino acids that are linked to each other in sequence by peptide bonds, when the chain of amino acids increases above 100 this amino acid chain is classified as a protein [1], while some others classify proteins as molecules with more than 50 amino acids in their sequence [2]. Peptides are selective and efficacious cell signalling molecules that bind to G-protein couple receptors (GPCR) or ion channels triggering intracellular responses [3]. Peptides have a role in regulating many physiological processes. Their functions include endocrine signalling, acting as neurotransmitters and acting as growth factors [4].

Peptides provide a number of advantages over more traditional small molecule agents, due to their increased number of interactions with the receptor site [5]. In comparison, small molecules which are less specific, can build up in non-specific organs. This can result in severe toxic side effects [6]. Peptides offer other advantages over conventional small molecule drugs such as high potency, high specificity, low toxicity and minimal drug-drug and nonspecific interactions [7, 8]. Table 1.1 highlights the advantages and disadvantages of peptide therapeutics.

Over the past decade peptides have been researched and utilised for a wide range of applications in medicine and biotechnology. One of the greatest developments that has helped this is the ability to produce peptides on a larger scale and in high quality. This has helped to reduce the overall cost and time of production. This allows for an easier method to produce hit analogues, speeding up the process from drug discovery 
Table 1.1: Advantages and disadvantages of peptide based therapeutic agents [3, 5, 6].

\begin{tabular}{cc}
\hline Advantages & Disadvantages \\
\hline High potency & Poor metabolic stability \\
High selectivity & Poor membrane permeability \\
Lower toxicity than small molecules & Rapid clearance \\
Low accumulation in organs & Poor oral bioavability \\
Lower number of drug-drug intreactions & Chemically and physically unstable \\
Shorter time to market & \\
\hline
\end{tabular}

to lead optimisation [9]. This has meant that peptides can be seen as a more realistic option as supply is able to meet demand.

The use of peptides in a medical environment is not a new phenomenon, peptide based therapy started almost 100 years ago with the use of bovine insulin for diabetes mellitus [10, 11]. There are currently more than 60 peptide based medicines approved by the US Food and Administration (FDA), with around 140 in clinical trials and more than 500 in preclinical testing [12]. The majority of these are small sequence peptides (8-10 amino acids long) [5]. In 2014, the global peptide market had been forecast to increase from US\$14 billion in 2011 to US\$ 25 billion in 2018, with the market for novel peptide to increase from US\$8 billion to US\$17 billion [13]. One of the reasons for this is due to the high success rate in target validation within the drug development stage compared to that of small molecules [14].

In the past few years, larger proteins and antibodies have also received much more attention [15]. However, peptides are smaller than proteins and antibodies. This provides some advantages, as they are easier to synthesise, do not cause immune responses and do not build up in the organs [6, 16]. 


\subsubsection{Vasopressin and Oxytocin}

The vasopressor effect of arginine vasopressin (AVP) was first reported in 1895 after discovering the extracts of cow pituitary had a vasopressor effect [17]. The uterotonic effect of oxytocin (OXT) was reported by Sir Henry Dale after he found an extract from the human posterior pituitary gland had a uterotonic effect [18]. This was followed by the work of Du Vigneaud in the 1950s, who discovered the structure of of AVP and OXT and the ability to chemically synthesise them [19, 20]. This became one of the first peptide hormones to be synthesised and won Du Vigneaud a Nobel Prize for chemistry in 1955 [21]. Since then, both AVP and OXT have been the focus of intensive research as both agonists and antagonists have various uses [22, 23, 24].

\subsubsection{Physiology}

AVP and OXT are synthesised in the neurons of the hypothalamus and are transported along their axons to the posterior pituitary. They are stored in the pituitary and released into the blood stream in response to detectors in the hypothalamus. AVP and OXT are 9 amino acids long, nona-peptides, with a disulphide bridge between two cysteines at amino acid 1 and 6. AVP and OXT differ from each other by two amino acids, in position 3 and 8. OXT contains isoleucine (Ile) and leucine (Leu) at position 3 and 8 respectively where as AVP contains phenylalanine (Phe) and arginine (Arg) at position 3 and 8 [25]. Figure 1.1 shows the structure and sequence of AVP and OXT, the differences in the structures are highlighted by the blue circles and squares.

Despite the similarities in their structure both AVP and OXT have very different effects in the body. AVP plays an important role in maintaining the body's fluid osmolality, blood pressure and adrenocorticotropin (ACTH) secretion [26]. AVP does this through receptors found at different sites in the body; the receptors are grouped together depending on their secondary messenger, either calcium $\left(\mathrm{Ca}^{+2}\right)$ for the vasopressin 1 receptor or cyclic adenosine monophosphate (cAMP) for the vasopressin 2 receptor $\left(\mathrm{V}_{2} \mathrm{R}\right)$. However both OXT and AVP show some binding affinity for their respective opposite receptors [27]. The binding affinity of each of AVP and OXT on 


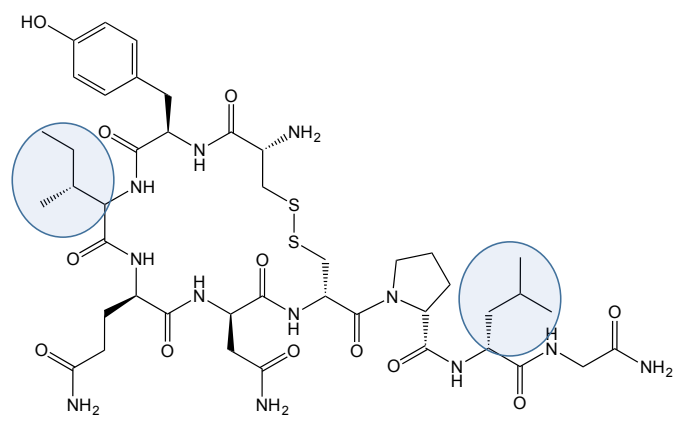

Cys-Tyr-lle-GIn-Asn-Cys-Pro-Leu-Gly- $\mathrm{NH}_{2}$

Oxytocin

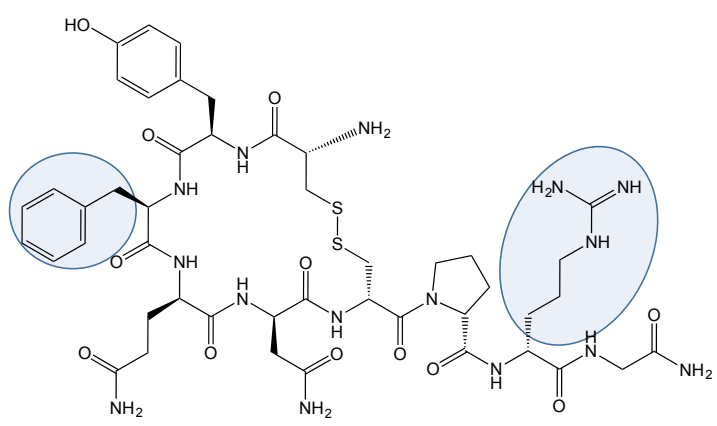

Cys-Tyr-Phe-GIn-Asn-Cys-Pro-Arg-Gly-NH Vasopressin

Figure 1.1: Structure and sequence of AVP and OXT, difference in sequence and structure are highlighted in blue

human AVP and OXT receptors is shown in table 1.2 .

Table 1.2: Binding affinity of AVP and OXT for human AVP and OXT receptors [28, 29]

\begin{tabular}{ccccc}
\hline & \multicolumn{4}{c}{ Binding Affinity (nM) } \\
\cline { 2 - 5 } & $\mathrm{V}_{1 \mathrm{~A}} \mathrm{R}$ & $\mathrm{V}_{1 \mathrm{~B}} \mathrm{R}$ & $\mathrm{V}_{2} \mathrm{R}$ & OXTR \\
\hline AVP & 1.7 & 1.1 & 1.1 & 1.65 \\
OXT & 64 & 167 & 1782 & 0.79 \\
\hline
\end{tabular}

The vasopressin 1 receptor is divided into two groups, vasopressin 1A receptors $\left(V_{1 A} R\right)$ and vasopressin $1 B$ receptors $\left(V_{1 B} R\right) . V_{1 A} R$ are found on the vascular smooth muscle, myocardial smooth muscle, platelets, and hepatocytes. When AVP activates $\mathrm{V}_{1 \mathrm{~A}} \mathrm{R}$ this results in vasoconstriction in both the coronary and peripheral circulation, platelet aggregation and gluconeogenesis [30]. The $\mathrm{V}_{1 \mathrm{~B}} \mathrm{R}$ is found in the anterior pituitary and when this activated by AVP this results in the release of ACTH. The role of $\mathrm{V}_{1 \mathrm{~B}} \mathrm{R}$ is not entirely understood, however animal experiments suggest it has a role in emotional behaviour [31].

$\mathrm{V}_{2} \mathrm{R}$ are found within the collecting duct tubules of the kidney and mediate the reabsorption of water. AVP results in the insertion of aquaporin-2 water (AQP2) chan- 
nels into the apical membrane of the collecting ducts. This then results in increased water permeability and an antidiuretic effect. This is why AVP is also commonly called anti-diuretic hormone (ADH) [32].

OXT acts on the oxytocin receptors (OXTR) found in the uterus, ovaries and parts of the brain [33]. Activation of the OXTR by OXT results in contraction of the uterine smooth muscle during labor and milk ejection during lactation [25]. Previously OXT was thought to be a female specific hormone, but more evidence is pointing to the role of OXT in males. It has been found that OXT is also synthesised in male testis, epididymis and prostate and plays a role in contractility, spermatogenesis and androgen metabolism in men [34].

\subsubsection{Development of Vasopressin Agonists}

Much time and effort has been used to develop agonist and antagonists for both AVP and OXT. Many reviews have covered the development these over the years [23, 22]. AVP agonists and antagonists can be used in conditions either linked to constriction of blood vessels or blood volume. The common use of AVP agonists is for the treatment of diabetes insipidus (DI). DI occurs due to reduced secretion of AVP and/or decreased activity of AVP [35]. Other conditions that can be treated with AVP agonists under current investigations and clinical trials are for are urinary incontinence, nocturnal enuresis; where the $\mathrm{V}_{2} \mathrm{R}$ is targeted [36] and septic shock where $\mathrm{V}_{1 \mathrm{~A}} \mathrm{R}$ is targeted [37]. AVP antagonists have uses in congestive heart failure $\left(V_{1 A} R\right)$, different types of hyponatremia $\left(\mathrm{V}_{2} \mathrm{R}\right)$, polycystic kidney disease $\left(\mathrm{V}_{2} \mathrm{R}\right)$ and some forms of hypertension $\left(\mathrm{V}_{1 \mathrm{~A}} \mathrm{R}\right)[30]$.

The first attempts at developing AVP agonist were performed by modifying the peptide structure of AVP. The agonists development had enhanced antidiuretic effect, improved plasma stability and a better selectively for $V_{2} R$ over $V_{1 A} R$ [38]. These studies lead to the discovery of desmopressin. Desmopressin differs from AVP by two modifications; deamino of the Cys at position 1 and substitution of L-Arg with D-Arg at position 8 [39]. 
Early structure activity studies had found that that the 20 member cyclic ring was essential for affinity for both AVP and OXT [40]. Other early studies also showed that the carboxy terminus (tripeptide tail) was essential for activity but not binding [41]. A summary of these are shown in figure 1.2 .

Some of the first modifications performed in Du Vigneaud's lab were preparing peptides with elements of both AVP and OXT to study the links between their chemical structure and biological properties. Arginine vasotocin (AVT) was the result of replacing Phe (position 3 of AVP) with Ile (position 3 of OXT). It was found that AVT had lower AVP activity [42]. This provided the information of the importance of Phe at position 3 in AVP agonists [43]. However, more recent studies by Stove et al. have found that some modifications at position 3 produced peptides with either similar or better activity than AVP. The modifications were made with various amino acids that have aliphatic $\mathrm{R}$ groups [44].

Other modification studies have been performed at position 2 of AVP, with substitution of Tyr with either bulky, aromatic or conformationally constrained groups, and these peptides were found to have little of no activity at the vasopressin receptors [45]. However, in another study the substitution of Tyr with 3,3-diphenyl-L-alanine or its D-enantiomer was shown to provide an improved antidiuretic effect with both the $\mathrm{L}$ and D-enantiomer's activity twice that of AVP [46].

Research into modifications at position 4 of AVP found this resulted in diminished $\mathrm{V}_{1 \mathrm{~A}} \mathrm{R}$ and $\mathrm{V}_{2} \mathrm{R}$ activity, but increased $\mathrm{V}_{1 \mathrm{~B}} \mathrm{R}$ activity. Cheng et al tested modifications of AVP at position 4. The amino acids at position 4 had been substituted for deamino acids with differing side chains. The affinity of these new peptides was tested for the AVP and OXT receptors. Experiments found that the length and branching of the aliphatic and aromatic side chain was a determining factor in affinity for the AVP receptors. Deamino Leu, Lys and non natural amino acids deamino Orn, Har and Cha were found to to be the best modifications at position 4 [47]. 


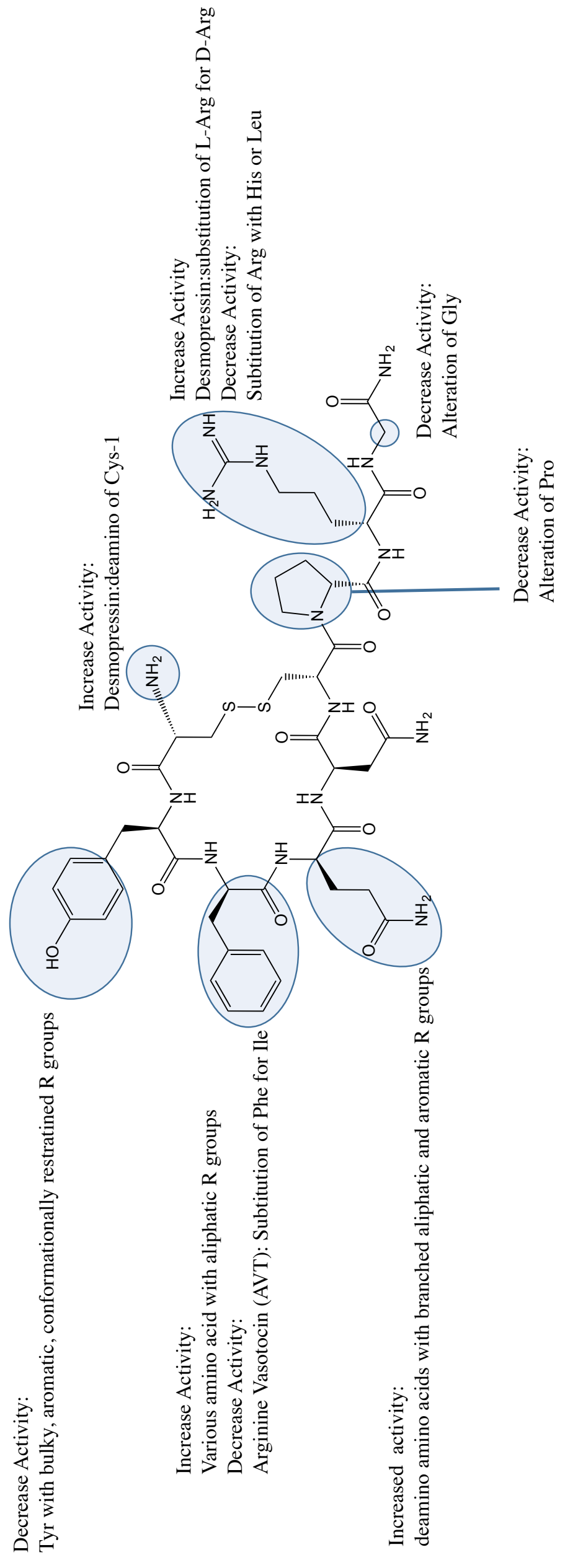

告 
Alterations of the proline at position 7 have resulted in AVP analogues that have reduced antidiuretic $\left(\mathrm{V}_{2} \mathrm{R}\right)$ and very little vasopressor $\left(\mathrm{V}_{1 \mathrm{~A}} \mathrm{R}\right)$ activities. This suggests that the proline has more of an effect on vasopressor activation than it does on antidiuretic activation [48]. Investigations into the importance of the tripeptide tail of AVP (Pro-Arg-Gly) were performed in a different study. The study found removal of Pro from position 7 or removal of both Pro and Gly from position 7 and 9 respectively resulted in analogues that have 1000 fold reduced affinity for the $V_{2} R$, showing the importance of both Pro and Gly for binding of AVP to the $\mathrm{V}_{2} \mathrm{R}$ [49]. This suggestion was further backed up with the substitution of tripeptide tail with bibasic dipeptide tail which resulted in a complete loss of activity [50].

Arg in position 8 plays a key role in AVP activation. Studies have found that its basic nature and length have a key role in receptor activation. Introduction of a less basic amino acid, His, meant the resulting peptide lost all AVP acvtivity. However replacing Arg with Leu, which has similar chain length resulted in reduced (60\%) AVP acitivity [51]. It is thought the length of the side chain and its basicity are critical for maximal receptor activation [52].

\subsubsection{Diabetes Insipidus}

DI is a rare condition caused by a reduced secretion of AVP and/or a reduced antidiuretic action of AVP [35]. The lack of AVP or its activity results in excessive thirst (polydipsia) and the production of large volumes of urine (polyuria). Polyuria can present as increased urinary frequency, nocturia, incontinence or enuresis [53]. Polyuria is defined by a urine volume in excess of approximately 100-110 ml per kg per 24 hr period until the age of 2 years and $40-50 \mathrm{ml}$ per $\mathrm{kg}$ per $24 \mathrm{hr}$ in older children and adults [54].

DI is a rare disease affecting 1 in 25,000 with central diabetes insipidus (CDI) the most common form [55]. There is no link between gender or ethnicity of patients [56]. There are two main types of DI, central and nephrogenic. CDI is due to a deficiency in AVP, lack of production and/or release of AVP into the blood. CDI is the result of irreversible destruction of the magnocellular neurons (MCN) either due to an 
acquired disease or genetic defect. Those with a genetic predisposition to CDI often have a defect in chromosome 4 or 20 [35, 53]. Nephrogenic diabetes insipidus (NDI) is caused due to a resistance to AVP [56]. NDI can be the caused by genetic mutation or acquired due to medication or a metabolic condition. Genetic mutations in either the vasopressin-2 receptor gene or aquaporin-2 gene are known to cause NDI [53].

Under normal conditions with normal fluid intake a person with urine in excess of $50 \mathrm{ml}$ per kg per day and with an plasma osmolality of less that $300 \mathrm{mOsm} \mathrm{L}^{-1}$ qualifies for diagnosis of DI [35]. The age at which the symptoms are observed may provide further insight into which type of DI it is [54]. In autosomal dominant CDI, the onset of the DI shows typically between the ages of 1 to 6 [57]. In CDI the symptoms show progressive polydipsia and polyuria if not treated [58]. To confirm diagnosis of DI a water deprivation and a desmopressin treatment trial may be performed. The urine osmolality with water deprivation and desmopressin treatment is compared to identify CDI or NDI [56]. Diagnosis of CDI is confirmed with plasma hyperosmolarity ( >300 mOsm L $\left.{ }^{-1}\right)$ associated with urine hypoosmolarity $\left(<300 \mathrm{mOsm} \mathrm{L}^{-1}\right)$ or a urine/plasma osmolality ratio $<1)$.

The drug of choice for the treatment of DI is desmopressin. Desmopressin is a AVP analogue that is more potent and longer acting than AVP. Desmopressin may be administered orally, intranasally or parenterally. It has a half life of 2-3.5 hour, having an effect within 1 to 2 hour after administration with a duration of action for up to 18 hour. The oral bioavailability of desmopressin is between 0.08 to $0.16 \%$. The daily dose for oral treatment is between 100 to $1200 \mu \mathrm{g}$ in three divided doses per day [54, 59]. Desmopressin is well tolerated by the majority of patients, but the risk of severe adverse effects, such as hyponatremia and water intoxication require the restriction of fluid intake [60]. Research into novel methods to treat and cure DI have included gene therapy to overcome the defects in the production of AVP, $V_{2} R$ and AQP2 water channels [61]. 


\subsection{Peptide Drug Delivery}

Therapeutic agents can be delivered by a number of routes; oral, sublingual and parenteral, topical. The route chosen will be depend on the properties of the therapeutic agent and the objective of treatment such as; rapid onset, duration of treatment, safety, patient satisfaction and cost [62]. The advantages and disadvantages of the various routes of administration are highlighted in table 1.3 .

Table 1.3: Route of peptide drug administration: advantages and disadvantages [62, 63].

\begin{tabular}{|c|c|c|}
\hline Delivery Route & Advantages & Disadvantages \\
\hline \multirow[t]{4}{*}{ Oral } & Convenient & $\begin{array}{l}\text { Peptides subject to harsh } \\
\text { environment of GI tract }\end{array}$ \\
\hline & High patient compliance & Limited bioavailability \\
\hline & Easily accessible route & $\begin{array}{l}\text { Presence of food can affect } \\
\text { absorption }\end{array}$ \\
\hline & $\begin{array}{l}\text { Absorption enhancers can } \\
\text { help delivery }\end{array}$ & $\begin{array}{l}\text { Pre-systemic elimination by } \\
\text { gut and liver }\end{array}$ \\
\hline \multirow[t]{2}{*}{ Subcutaneous } & $\begin{array}{l}\text { Can be administered by patient } \\
\text { or carer }\end{array}$ & $\begin{array}{l}\text { Requires small volume } \\
(<2 \mathrm{~mL})\end{array}$ \\
\hline & $\begin{array}{l}\text { Less painful than IV or IM } \\
\text { Low risk of systemic infection } \\
\text { Large number of injection sites } \\
\text { Long retention time at injection } \\
\text { sites }\end{array}$ & May degrade at injection site \\
\hline \multirow[t]{2}{*}{ Intravenous } & Allow for large volume & Painful \\
\hline & $\begin{array}{l}\text { Can be used for drugs not } \\
\text { readily soluble }\end{array}$ & $\begin{array}{l}\text { Risk of blood or nerve } \\
\text { damage ( } 39 \% \text { incidence) } \\
\text { Risk of infection, bleeding, } \\
\text { abscess formation } \\
\text { Requires a trained professional } \\
\text { Increased cost of administration }\end{array}$ \\
\hline
\end{tabular}




\subsubsection{Oral Delivery of Peptides}

In 2003 the World Health Organisation (WHO) issued a report on adherence to long term therapies. They recognised poor adherence to medications as a problem for chronic diseases all around the world. The WHO estimated that only $50 \%$ of patients in developed countries were adherent and the figure for developing countries was much less [64]. Poor adherence compromises the efficacy of long term treatment resulting in a reduced quality of life, increasing mortality and places a greater burden on the healthcare system [65, 66, 67]. Oral drug administration is the most convenient and preferred medication route [68, 69]. This improved convenience aid to increase adherence with the dosing regimen prescribed leading to increased therapeutic efficacy [69].

Oral delivery of medications is the most common route of administration accounting for nearly $40 \%$ of all administered medications. This is followed by pulmonary drug delivery of medications making up $20 \%$, followed by transdermal method third and injectable method fourth [70, 71]. More than $60 \%$ of small molecule drugs are administered orally [72]. It is also the preferred delivery route by the pharmaceutical industry as it easiest and cheapest to manufacture, not requiring sterile conditions [73, 74].

\subsubsection{Barriers against Oral Delivery}

The overall function of the barriers within the gastrointestinal (GI) tract is to protect the interior of the body from a range of foreign toxins and foreign particles. The skin is the largest barrier whereas the blood brain barrier is the considered to be the toughest barrier to pass through [75, 76]. In order for a peptide to be orally bioavailable it must pass into the systemic circulation once orally administered, and using knowledge of the nature of these barriers will help to make an orally bioavailable peptide formulation that can tackle these barriers [77].

The oral bioavailability of peptide formulations is limited primarily by limited absorption and permeation across the GI tract and enzymatic degradation within the GI 
tract [78]. The GI tract maybe divided into two regions, the upper made up of the mouth, oesophagus and the stomach. The lower region is made of up of the small and large intestine. The absorption of proteins and peptides is limited in the stomach due to the low surface area, degradation by pepsin and the acidic environment. Within the small intestine more than $90 \%$ of nutrients such as carbohydrates, protein, lipids, vitamins and minerals are absorbed. The microvilli within the small intestine provide an increased surface area for absorption to to take place [79].

The barriers against oral drug delivery may be divided into two major groups the physiological and biochemical barriers. The physiological barriers are made up of the impermeable GI tract. The biochemical barriers cause the degradation of proteins and peptides by peptidases [80].

\subsubsection{Physiological Barriers}

One of the key functions of the physiological barrier is to provide protection against the passage of toxins, bacteria and viruses into the systemic circulation [81]. The lumen of the GI tract is covered with aqueous mucus which is secreted by goblet cells. This mucus is made up of glycoproteins that trap water. The thickness of mucus varies along the GI tract with the thickest in the stomach and colon [82]. It can also vary due to diet [83]. The number of goblet cells varies along the intestine [84]. The mucus acts like a filter for molecules with a mass of 600-800 Da [85]. The penetration through the mucus is considered to be the rate limiting step for a peptide to reach the surface of enterocytes for absorption [85]. Below the mucus there is a single layer of columnar epithelial cells that are held together by tight intracellular junctions, which forms the barrier between the systemic circulation and the lumen of the GI tract. The layers of cells are made up of enterocytes, goblet cells, endocrine cells and paneth cells [86].

A drug can cross this layer by different mechanisms depending on its physiochemical properties [87]. Hydrophobic drugs are able to partition through the cell membrane and so are more likely to pass through to the systemic circulation via the transcellular pathway. However, the phenomenon seen with peptides is different to that of small molecules. Peptides ability to pass via this route is determined by the num- 
ber of polar groups that require desolvation before a peptide can diffuse into the cell membranes [88].

In the late 1990s Lipinski et al. research showed that more than $90 \%$ of oral deliverable drugs had 4 common properties; a molecular mass of less than 500, calculated octanol/water partition coefficient ( $\operatorname{cog} \mathrm{P})$ less than 5 , the number of hydrogen bond donors less than 5 and number of hydrogen bond acceptors less than 10. This became known as Lipinski's rule of 5 [89]. However there are a few orally delivered peptide based drugs on the market that break this rule, this means there is some flexibility on the Lipinski's rule of 5. Santos et al. studied orally bioavailable peptides to enhance these guideline for peptides. After systemically reviewing new peptides approved by the FDA between 2012-2016, they found that a Mw up to $700 \mathrm{Da}, \log \mathrm{P}$ of up to 7.5 and number of hydrogen bond donors less than 5 could be orally bioavailable. The authors also commented that these boundaries could be pushed further, if peptides are able to behave more like small molecules; with regards to the surface area they take up when they are dissolved in the GI tract [90].

PepT1 on the apical membrane is one of most common peptide paracellular transporters, with the PepT2 transporter allowing for the substrate to exit the enterocyte into the systemic circulation. PePT1 and PePT2 transport dipeptide and tripeptide based drugs across the intestinal membrane, with the concentration of transporters increasing from the duodenum to the ileum. During periods of starvation the expression of these pumps increases to aid a higher uptake of dipeptides [91].

Hydrophilic drugs cannot pass into the cell membranes so they must pass into the systemic circulation via the paracellular pathway, in between the cells [92]. This pathway is restricted by size due to the presence of tight junctions, only those molecules with a hydrodynamic radius less than $11 \AA$ can pass through [93].

\subsubsection{Biochemical Barriers}

An essential function of the gastrointestinal tract is to break down ingested macromolecules such as proteins and peptides into small enough subunits so that they can be 
absorbed. This metabolic barrier is considered to be one of the most critical barriers limiting the absorption of intact peptide drugs [78]. Most peptides are liable to rapid degradation by the digestive enzymes [94].

Peptidases are enzymes that hydrolyse the bond between two amino acids in a protein or peptide chain. Peptidases can be further divided into two groups; endopeptidases and exopeptidases. Endopeptidases hydrolyse the bonds in the inner parts of a peptide chain, whereas exopeptidases hydrolyse the bonds on the terminal parts of a peptide chain. The exopeptidases can be further divided into two further groups; carboxypeptidases which break down the bonds on the carboxy terminal of the peptide chain and the aminopeptidases which break down amide bonds on the amino end of the peptide chain [95].

Proteolytic degradation is greatest in the stomach and duodenum, with a reduced amount taking place in the ileum and colon [10]. The first metabolic barrier peptides are exposed are in the stomach. The stomach contains a combination of proteolytic pepsin and hydrochloric acid $(\mathrm{HCl})$, this results in protein hydrolysis. However, smaller peptides are more stable in this environment [85].

The enzymes of the upper small intestine are considered the second barrier [85]. The pancreatic proteases of the duodenum have the highest activity at $\mathrm{pH}=8$, breaking down large proteins to small peptides within 10 minutes. The smaller peptides are broken down by proteases found on the brush border and in the cystol of enterocytes[85].

\subsubsection{Subcutaneous Delivery}

There are some medical conditions where oral delivery is not an option, in which a parenteral is the only option. For example, Type 1 diabetes mellitus till very recently could only be treated with parenteral insulin [96], but a new insulin inhaler has been developed and received marketing authorisation by the FDA for USA in 2014 [97, 98]. Subcutaneous (SC) delivery offers a convenient parenteral method of administration compared to intravenous (IV) delivery. SC injections have been approved to deliver a number of therapeutic peptides such as insulin and growth hormones [99]. Lantus 
(insulin glargine) is the most common medication delivered by SC injection, it is the $6^{\text {th }}$ highest selling medication by sales and the $9^{\text {th }}$ highest selling medication by revenue in USA during 2014-2015 [100]. A systematic review by Stoner et al. found that patients prefer SC over IV delivery. Between $44-91 \%$ of patients preferred the SC over the IV route of administration. Patients preferred the SC route as they are able to have the treatment at home. This saved time at hospital, avoided complications with IV administration or venous access [101], and reduced discomfort [102].

Aside from just the patient benefits of SC delivery, the medical cost of SC delivery is less than that of IV. Rule et al. performed a study into the cost of the administering rituximab by IV or SC for the treatment non-Hodgkin lymphoma [103]. The study found that SC administration saved nearly 3 hours of direct patient contact time with the health care professional (HCP). This resulted in the hospital saving $£ 115$ per patient per session of treatment. Patients also spent less time in hospital, 303 vs. 110 minutes for IV and SC treatment respectively, saving more than 3 hours per session [103]. Similar findings were also reported by Jin et al. in a literature review of published findings of which route IV, IM or SC is preferred. Jin et al. found that if the treatment efficacy is similar; SC is the preferred route of administration [104].

\subsubsection{Physiology}

SC injections deliver the contents of an injection into the interstitial space of the hypodermis. The hypodermis varies slightly from species to species [99, 105]. The human hypodermis is made up of fat lobes called adipose tissue which are separated by loose connective tissue [106]. Adipocytes are the main cells found in the hypodermis, but there are also a smaller amount of fibroblasts and macrophages. Fibroblasts produce the components of the extracellular matrix (ECM) [107]. The function of ECM is to provide mechanical stability by forming a three dimensional network [108]. The ECM is made up of collagen, hyaluronic acid (HA), glycosaminoglycans (GAG) and the interstitial fluid [109].

The mechanical stability of the ECM is provided due to the presence of collagen. Collagen assists to provide stability in other areas of the body such a tendons and 
basement membranes as well. There are 21 different types of collagen, which are grouped together based on their structure and supramolecular organisation [110]. The SC tissue contains type I, III and V collagen with a higher ratio of type III [111]. The collagen fibres are bound into bundles that are between 1-100 $\mu \mathrm{m}$ thick [109]. Between the voids of collagen HA is found. The function of HA is to help maintain fluid balance, regulate the distribution of proteins by steric exclusion and help to lubricate tissues [112]. The GAG of the ECM is made up of chondroitin sulphate, a linear glycosaminoglycan polysaccharide, with a molecular weight of between 30-50 kDa. The function of GAG within the ECM is to help maintain the $\mathrm{ECM}^{\prime}$ viscosity and fluid balance [113].

The interstitial fluid makes up nearly $25 \%$ of total body water [114] which is perfused within the SC space. The $\mathrm{pH}$ of interstitial fluid is tightly regulated to be within $\mathrm{pH} 7.35$ to 7.45 [115]. The $\mathrm{pH}$ is regulated by the strong ion difference and the partial pressure of carbon dioxide in the tissue [116]. The major cation in interstitial fluid is sodium and the major anion is chloride, with the concentration of sodium higher that that of cholride, this provides the strong ion difference. The production of carbon dioxide $\left(\mathrm{CO}_{2}\right)$ from cell metabolism helps to decrease the $\mathrm{pH}$ as the dissolving of $\mathrm{CO}_{2}$ forms bicarbonate $\left(\mathrm{HCO}_{3}{ }^{-}\right)$and carbonate $\left(\mathrm{CO}_{3}{ }^{2-}\right)$ ions to reduce the positive strong ion difference and raise the $\mathrm{pH}[109]$

\subsubsection{Factors affecting Subcutaneous Delivery}

SC injections bypass the dermis and epidermis of the skin and deliver the therapeutic agent to the extracellular space of the SC tissue, hypodermis. From the SC tissue the therapeutic agent will be transported to the systemic circulation by either the capillaries or the lymphatic system [105]. The factors determining the delivery of the therapeutic agent after SC injection can be categorised as the physiological factors of the tissue and the physiochemical properties of the peptides [105]. 


\subsection{Physiological Factors}

\section{Site of Injection}

The site of injection is important as the thickness of SC tissue is not the same all over the body. The site of injection does not affect the overall bioavailability, but will affect the rate of absorption from the injection site. A higher rate of absorption was observed when studying the effect of injecting insulin in the abdomen compared to the arm or thigh. An interesting finding found that the slower absorption also resulted in a longer duration of action, which would have implications on therapeutic outcome [117]. Similar results were also seen with growth hormone absorption and the site of injection [118]. The differences in rates of absorption from the site of injections is suggested to be due differences in SC blood flow of the different regions. Those sites with greater blood flow show greater a rate of absorption [118].

The thickness of the hypodermis varies not only on the site, but also from person to person. A study into the thickness of SC tissue in arms, thighs, abdomen and buttocks found that SC tissue varies more by body site, gender and body mass index (BMI) than it does by race or age [119]. Females had thicker SC tissue than men in all four areas studied. Also it was found that buttock has the thickest SC tissue, followed by abdomen. The arms and thigh had the a similar thickness of SC tissue. As BMI increased so did the thickness of SC tissue [119].

\section{Transport from SC Injection Site}

After SC injection the therapeutic agent needs to transported into the systemic circulation so it can reach the site of action. Macromolecules (less than $16 \mathrm{kDa}$ ) mostly pass from the SC injection site to the systemic circulation by diffusion into the blood capillaries. Larger molecules travel through the lymphatic system [105]. The lymphatic capillaries have a higher permeability to larger molecules as they do not have a well-defined basement membrane and lack tight cell junctions. This less defined basement membrane results in lower resistance of fluid flow compared to blood capillaries 
[120]. Once larger molecules pass into the lymphatic capillaries the molecules then pass into the lymph nodes, then to larger lymphatic vessels and eventually pass to the thoracic and other lymph ducts and the systemic circulation [121]. Transport through the blood capillaries or the lymphatic system to the systemic circulation could a rate limiting factor to absorption. The process of transport results in delayed delivery times. This delivery time is determined by the hydrostatic and osmotic differences that occur within the blood, interstitium and the lymphatics [105]. Studying the uptake of insulin via the blood capillaries or lymphatic system in sheep, it was found that both contributed to a similar degree of transport into the systemic circulation [121].

\subsection{Physiochemical Properties of Peptide}

\section{Molecular Size and Weight}

Particles smaller than $10 \mathrm{~nm}$ are transported to the systemic circulation via the the blood capillaries, whereas particles that are $10-100 \mathrm{~nm}$ in size are transported to the systemic circulation via the lymphatic system. Studies have found that smaller particles have a faster uptake [120]. Although an upper limit has not been suggested for SC delivery; as microparticle have been delivered subcutaneously [122], it is thought particles larger than $100 \mathrm{~nm}$ in size will mostly remain at the SC tissue [123].

Supersaxo et al. studied the lymphatic absorption of peptides and proteins ranging from $200 \mathrm{Da}$ to $19 \mathrm{kDa}$ after $\mathrm{SC}$ injection in sheep. A linear relationship between the molecular weight to percentage of the dose that was recovered from the lymphatic system was found [124]. However, other studies have found different results in rats. A study of lymphatic absorption of insulin, bovine serum albumin (BSA) and erythropoietin found that less than $3 \%$ was absorbed by via the lymphatic system [125]. The literature shows there is a species to species difference with regard to lymphatic uptake [105]. 


\subsection{Charge}

The ECM is made up of collagen fibres and GAGs. The negative charge of the GAG helps to control fluid balance [120]. At physiological $\mathrm{pH}$ the collagen and HA have a negative charge [109]. This negative charge density provides a barrier to the transport of macromolecules from the site of injection to the systemic circulation [105]. Negatively charged molecules or particles pass through the SC space faster than neutral molecules and particles due to electrostatic repulsion [107]. One study investigated the transport of charged dextrans through the SC tissue, it found negatively charged dextrans was delivered faster than neutral dextrans [126]. Another study investigated the transport radiolabeled charged liposomes. This study found that negatively charged liposomes passed through the interstitial space quicker. This was followed by positively charged liposomes and the slowest was neutral liposomes [127]. The effect of charge affects the speed at which the molecule or particles passes through the SC tissue, with negatively charged molecules or particles passing through the fastest due to repulsion. This is followed by positively charged and the slowest are neutral molecules and particles [128]. 


\subsection{Methods to Overcome Factors for Peptide Delivery}

The approaches currently being used and investigated to develop a peptide delivery system may be divided into two main groups; 1) modification of the physicochemical properties of the peptide, 2) use of a delivery system [129]. Many technologies have started to be evaluated in clinical trials, figure 1.3 shows the various technologies and the stages of clinical trials they are currently at.

\subsubsection{Chemical Modification}

Chemical modifications of the peptide can either entail conjugation of the peptide with another compound or modification of the peptide itself. The main reasons for chemical modification is increased enzymatic stability and improved intestinal permeability [130]. A certain degree of lipophilicity is required for molecules to pass into the epithelial cells and then for the molecule to partition out of the epithelial cell into the systemic circulation [131]. Chemical modifications may include forming analogues by substitution of amino acids, acylation or PEGylation [7].

The covalent conjugation of a peptide to a hydrophobic compound may help to increase the lipophilicity whereas attaching a poly(ethylene glycol) (PEG) moiety will increase the aqueous solubility as in order for a drug to be orally absorbed it needs to be in solution in the GI tract [132] and protected against enzymes [133].

Direct conjugation of PEG can help to improve peptide solubility aiding an improvement in peptide absorption and it can also help prevent peptide degradation due to steric hinderance at the enzyme active site [134]. One such example is the conjugation of PEG to salmon calcitonin ( $\mathrm{sCT}$ ), this resulted in a significant better hypocalcemic effect compared to unconjugated SCT. The increased effect of conjugated $\mathrm{sCT}$ was found to be due to a increased intestinal stability and a reduction in systemic clearance [135].

One of the most successful chemical modifications of a peptide is the formation of desmopressin from AVP. AVP has very little oral activity, due to its instability in 
the GI tract and therefore is only be administered intravenously. Oral desmopressin is around 50 times more orally active than oral AVP, which is due to its increased stability within in GI tract and plasma, which also results in a prolonged duration of action [136]. Desmopressin differs from AVP by two modifications; deamination of the terminal amino acid and the replacement of L-Arg at position 8 with D-Arg [137].

In some molecules certain chemical modifications may reduce biological activity and so a prodrug can be developed. Prodrugs are pharmacologically inactive entities made by chemical modification [7]. Prodrugs are a bio-reversible derivate of the drug molecule, that undergoes an enzymatic or chemical transformation in vivo to release the active parent drug. Prodrug technology has been identified as a tool for improving the physiochemical, biopharmaceutical or pharmacokinetic properties of pharmacologically active agents. Around 5\% of approved drugs in the market are prodrugs [138]. This approach has been used to improve the oral absorption of leu-enkephalin (LENK), an endogenous enkephalin. Lalatsa et al. developed TPLENK, a palmitic ester prodrug of LENK, esterase activity would utilised to re-generate LENK from TPLENK. TPLENK showed greater plasma stability than LENK. Orally delivered TPLENK using a nanoparticle system TPLENK showed significantly increased anti-nociceptive activity [139].

A similar approach to desmopressin was also applied to LENK. L-Ala was replaced with D-Ala at position 2 and adamantine moieties were attached via an ester bond at the $\mathrm{C}$ terminus. The prodrug delivered via a SC injection showed enhanced antinociception in male mice, however the D-Ala LENK without the adamantine showed no activity when delivered by SC injection [140]. Another study found that modifying the C-terminus with $\mathrm{D}$-amino acids improved the stability of peptides against proteases. The unmodified peptides were degraded quickly, whereas the D-amino acid modified peptides were stable up to 24 hours [141]. However, a problem faced with D-amino acid modified peptides is a reduced affinity for the desired receptors and reduced cellular uptake, this has limited research with this modification [142].

Another modification approach has been to conjugate peptides with moieties such as vitamin $\mathrm{B}_{12}$ or folate to increase oral uptake. One study studied the site of conjugation of vitamin $\mathrm{B}_{12}$ to insulin, two sites were chosen; Phe at B1 and Lys at B29. 
Studying their effect on blood glucose in comparison to unmodified oral insulin, both conjugates resulted in a reduced of blood glucose. Both conjugates caused a drop in blood glucose of about 30\% within 60 minutes which was sustained for a further 3 hours. The two conjugates had the same extent of effect, so neither could be considered a better site of conjugation [143]. However, development of these conjugates has been limited as vitamin $B_{12}$ uptake has a limited capacity and is relatively slow in the GI tract, meaning a clinically relevant dose cannot be administered [144]. 


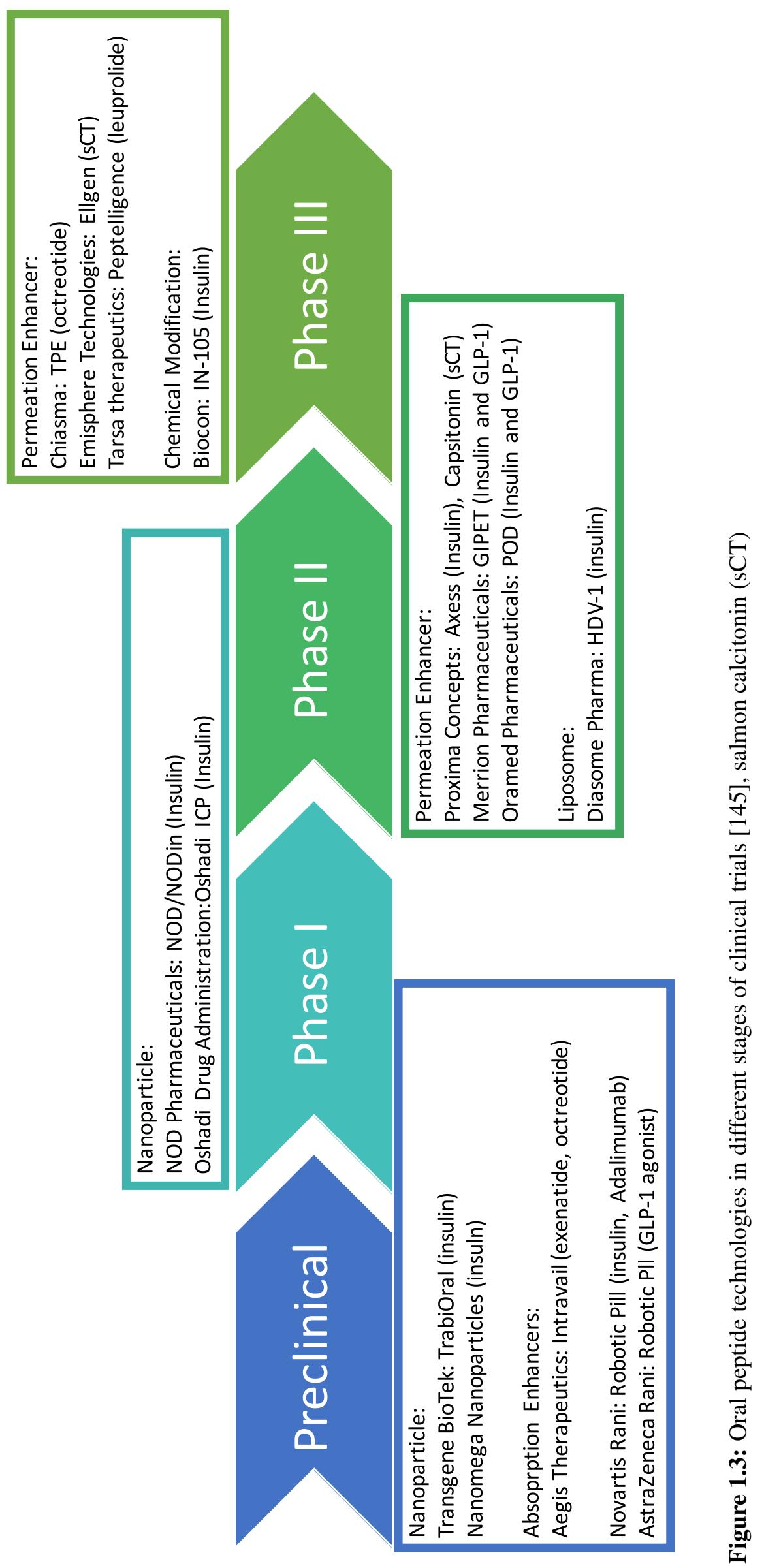




\subsubsection{Protease Inhibitors}

One of the biggest barriers in oral peptide delivery is the degradative enzymes [78]. To limit the effect of enzymatic attack various researchers have tried to use enzyme inhibitors [146]. Protease inhibitors can be grouped depending on if they amino acid based and non-amino acid based inhibitors. Amino acid based include soybean trypsin inhibitor, sodium glycocholate, camostat mesilate whereas non-amino acid based protease inhibitors are agents such as calcium, zinc, cobalt, manganese and magnesium [147]. Newer class of enzyme inhibitors has been developed derived from chicken and duck ovomucoids.

One such example has been the use of casein protein derived from milk to deliver insulin. This formulation was delivered orally and found to improve blood glucose control in diabetic mice. It was found to a have reduction in blood glucose for a period of 8 hours [148]. In another study, the use of a duck ovomucoid helped to provide a better decrease in blood glucose in comparison to an unprotected oral tablet of insulin. This approach however was not as effective as SC insulin. The concentration of ovomucoid used could not be increased due to toxicity concerns [149]. Toxicity has limited the number of in vivo studies that have taken place. The toxicity of these enzyme inhibitor formulations, their influence on the absorption of other key proteins and what may be the long term effects on the use of these types of inhibitors is a concern among researchers and clinicians [150].

However, some enzyme inhibitor based products have started to reach clinical trials. Oramed Pharmaceuticals (Israel) have developed Protein Oral Delivery ( $\mathrm{POD}^{\mathrm{TM}}$ ), an enteric coated capsule that contains a suspension of peptide or protein with an soy bean trypsin inhibitor [151]. Their lead candidate, ORMD-0801, has been tested in 5 Phase II clinical trials (NCT02496000, NCT02094534, NCT00867594, NCT01889667 ongoing; NCT02535715). In pilot studies ORMD-0801 showed a significant 16\% decrease in blood glucose over 24 hours, with a $24 \%$ reduction in the number of glucose readings above $200 \mathrm{mg} \mathrm{dl}^{-1}$ [152].

Another company, Enteris Biopharma Inc. (USA) have formulated a salmon cal- 
citonin ( $\mathrm{sCT}$ ) enteric coated tablet. The tablet consists of maltodrexin coated citric acid granules and an absorption enhancer, Eudragit ${ }^{\circledR}$. The citric acid granules prevent the contents of the tablet being broken down by degradative enzymes, as the lower the $\mathrm{pH}$ reduces the effectiveness of proteolysis enzymes [153]. A phase 2 study has been planned but is not recruiting yet. The Phase II study will provide pharmacokinetic/pharmacodynamic effects from a multiple dosing 28 day study (NCT02807363), but no details of the Phase I study could be found on the clinical trial database.

\subsubsection{Absorption Enhancers}

\subsubsection{Permeation Enhancers}

Permeation enhancers help to increase permeability rather than affecting molecular solubility. Oral absorption enhancers act by a number of mechanisms; temporarily disrupting the structural integrity of the intestinal barrier, decreasing mucous viscosity, opening tight junctions and/or increasing membrane fluidity [154, 155], allowing the therapeutic agent to cross into the systemic circulation.

They can be grouped into surfactants, chelating agents, bile salts, cationic and anionic polymers, acylcarnitines, fatty acids and their derivatives [84]. An ideal permeation enhancer has three major requirements; significant but temporary improvement in peptide absorption, intestinal barrier recovery and be safe to use in humans [156].

Using this approach some researchers have developed oral peptide formulations for human clinical trials. Gastrointestinal permeation enhancement technology (GIPET) developed by Merrion Pharmaceuticals (Ireland) a product that uses short fatty acid chains to deliver drugs that have a bioavailability of less than 1\% [157]. Merrion Pharmaceuticals have partnered with Nordisk (Denmark) to develop an oral insulin product that is currently in clinical trials . Phase I studies (NCT01931137, NCT02304627, NCT01809184 and NCT01796366) were performed with an enteric coated gel capsule, no results could be found for these studies. This formulation moved in a 8 week long Phase II study (NCT02470039) with multiple dosing, which completed 
in November 2015. No results have been reported for the phase II study either.

GIPET, is an enteric coated gel capsule that is made up of a mixture of fatty-acid derivatives, has also been used in a Phase I clinical study (NCT00471185) for the delivery of acyline for the treatment of prostate cancer. Acyline is a potent gonadotropinreleasing hormone antagonist [158] that results in the suppression of testosterone production [159]. The Phase I study found significant suppression of testosterone levels in the body, with a 20 and $40 \mathrm{mg}$ dose having an effect for 24-48 hours [158].

Although these methods are shown to be successful in the laboratory and have started to reach early clinical trials, clinicians and regulatory bodies are still cautious. They are concerned about their long term use as it may allow the absorption of unwanted proteins, xenobiotics and pathogens as the permeation enhancers are not peptide specific [156].

Permeation enhancers may also be used to improve SC delivery. Recombinant human hyaluronidase can be injected with a peptide to degrade HA which can help with peptide and protein delivery. The HA degradation will help to increase the diffusion rate at the injection site, which can increase the rate of bioavailability and also the degradation of HA will allow for an increased injection volume [160]. A study compared the difference between delivering insulin with and without recombinant human hyaluronidase, both were found to have a similar efficacy over a 12 week period. However, the recombinant human hyaluronidase insulin dosed patients had a overall tighter control over bloody glucose throughout the day [161].

Another permeation enhancer oral peptide delivery system that has reached clinical trial stages is developed by Emisphere Technologies (USA). It is made up of a number of $\mathrm{N}$-acylated alpha-amino acids with intestinal PE properties called Eligen ${ }^{\circledR}$ [151]. In partnership with Novo Nordisk, Eligen is being used to develop a product that deliver GLP-1 agonist for diabetes mellitus. This has entered a Phase II study (NCT01037582), tested in 600 type 2 patients for 26 weeks. Results showed the product was well tolerated with plasma $\mathrm{Hb} 1 \mathrm{AC}$ level were reduced by $1.9 \%$, achieving the primary end point [162] 


\subsubsection{Cell Penetrating Peptides}

Another class of absorption enhancers are cell penetrating peptides (CPP). CPPs are a chain of less than 20 amino acids that are internalised into cells. Although there are many different primary and secondary structures of the various CPPs, they share some similarity that they have a large number of basic amino acids such as Arg and Lys. These basic amino acids allow interactions with the negatively charged cell surface molecules of plasma membrane [163]. They are grouped together as either cationic, amphipathic, or hydrophobic peptides [164]. Some examples are HIV-1 Tat, penetratin and oligoarginine [74].

Both endocytosis and direct translocation are believed to be involved in the internalisation of CPP formulations [165]. However, there are many factors that determine which uptake mechanism takes place; this is dependent on the amino acid sequence, the CPP concentration and any modifications of the natural CPP [166]. For example one study found that when Arg rich CPP was to deliver low concentrations of peptide (2- $10 \mu \mathrm{M})$ the peptide CPP formulations were internalised via endocytosis. However, in larger concentrations of peptide $(20 \mu \mathrm{M})$ the CPP formulations were internalised using by direct translocation [167].

The quest to administer insulin orally has been one of the most researched area in the oral delivery of peptides. In order to enhance the efficacy of penetratin, four positive charged modifications of penetratin were studied to deliver insulin. The CPP formulations were found to result in up to $50 \%$ decrease in blood glucose over 60 minutes, whereas unprotected insulin did not result in any changes to blood glucose [168]. However, every CPP will not be appropriate for every peptide, this mean each CPP has to individually evaluated for each peptide [169]. This is shown in a study that tested the use of oligoarginine and penetratin to deliver IFN- $\beta$. Oligoarginine was selected as it has previously shown to assist in the delivery of insulin [170]. It was found that oligoarginine did not produce an improvement in the oral delivery of IFN- $\beta$ whereas penetratin did significantly improve its delivery. The authors suggested this could be because penetratin is more hydrophobic than oligoarginine and secondly as IFN- $\beta$ has a positive charge within the intestinal lumen this may limit the intermolecu- 
lar interactions with oligoarginine which is positively charged. This repulsion results in a reduced attraction between IFN- $\beta$ and oligoarginine [171].

\subsubsection{Particulate Carrier Delivery Systems}

\subsubsection{Polymeric Carries}

\subsection{Hydrogels}

Hydrogels are materials made from hydrophilic polymers. They are insoluble in water at physiological temperature and $\mathrm{pH}[172]$. They also retain a great amount of water which helps to increase the integrity of their system [121]. Hydrogels have generated great interest in the oral delivery of insulin [173].

One group has used poly(acrylic acid-co-acrylamide)(O-carboxyemthyl chitosan) to help protect and deliver insulin. Studies were used to monitor drug release, stability and then tested in vivo to control blood glucose level. During in vitro studies less than $20 \%$ of insulin was released at gastric $\mathrm{pH}(\mathrm{pH} 1)$ over 2 hours whereas at $\mathrm{pH} 7$ nearly $100 \%$ of insulin was released within 1 hour. The hydrogel also helped to protect insulin from enzyme degradation for over 2 hours with bare insulin becoming fully degraded within 1 hour. During in vivo experiments the hydrogel helped to maintain a 60\% drop in blood glucose over 6 hours [174].

An enhancement of conventional hydrogels has resulted in newer smart drug delivery systems. These systems utilise polymers with modifications such that they respond to specific stimuli of the environment. For example they can be contracted at low $\mathrm{pH}$ (gastric $\mathrm{pH}$ ) but swell at neutral $\mathrm{pH}$ (intestinal $\mathrm{pH}$ ) enabling drug release. This allows the hydrogel to release its drug contents in specific conditions, resulting in the term intelligent hydrogels. They can respond to other stimuli such as temperature, electric field, magnetic field or the ionic strength of a medium [175].

One such example is the use of a pH sensitive chitosan based hydrogel to deliver insulin. The chitosan based hydrogel swells in intestinal pHs releasing insulin, whereas 
the hydrogel remains contracted in gastric $\mathrm{pHs}$ protecting insulin. In in vitro studies it was found that the $\mathrm{pH}$ sensitive hydrogels released between $50-100 \%$ of insulin in the intestine environment, where as the native chitosan hydrogel only released $20 \%$. In in vivo studies the insulin delivered using the $\mathrm{pH}$ sensitive hydrogels showed a larger drop in blood glucose compared to the native chitosan hydrogels [176].

\subsection{Micro and Nanoparticles}

Both micro and nanoparticles have been intensively studied for the oral and SC delivery of peptides [177]. Micro and nanoparticles are made from biodegradable, biocompatible and nontoxic polymers such as polylactic acid (PLA), polylactic coglycolic acid (PLGA), polycaprolactone (PCL). Other polysaccharides such as chitosan and alginate are also used for delivery of peptides and proteins [178].

\section{Microparticles}

Microparticles are particles that are above $1 \mu \mathrm{m}$ in size and help to increase the bioavailability of peptides by encapsulating them and providing protection from the harsh environment of the GI tract. This approach has been used to create a formulation that has the ability to lower blood glucose over a five hour period [179]. Sodium alginate and mucin were used in different ratios to produce insulin loaded microparticles. Others have used PLGA microparticles to research the delivery of GLP-1. Researchers have produced microspheres containing GLP-1, a hormonal treatment for diabetes, which was delivered orally and provided an improved glucose tolerance over 8 hours compared to unencapsulated GLP-1 [179]. Unencapsulated GLP-1 was not able to provide any drop in blood glucose at any time point over 8 hours [179].

\section{Nanoparticles}

Nanoparticles are defined as particles ranging in size from 10 to $1000 \mathrm{~nm}$ [180]. Nanoparticles have shown great promise in the delivery of peptides, proteins and antibodies 
[181].

One such example is the use of a glycol chitosan based polymer. Quatnery ammonium glycol chitosan (GCPQ) has been used to deliver a wide range of therapeutic agents. One such example is the delivery of LENK to the brain after oral administration. LENK encapsulated within GCPQ was able to provide a sustained response over 5 hours compared to unencapsulated LENK, which was not able to provide any response. This resulted in more than 4 times more anti-nociception over a period of more than 5 hours compared to unencapsulated LENK [139]. Studies have shown that GCPQ formulations adhere to the mucosa in the GI tract after oral administration [182]. This allows the GCPQ nanoparticles to be closer to the enterocytes. GCPQ nanoparticles pass through the enterocytes into the portal system prior to the systemic circulation [183].

Another example is the use of PEG coated PLGA nanoparticles to improve the delivery of insulin. The resulting nanoparticles were under $200 \mathrm{~nm}$ in size and allowed for a decreased glucose level over 24 hours, with a peak drop in blood glucose taking place at 3-6 hours. The highest insulin level was seen at 6 hours post dosing [184].

Nanoparticles have also been investigated for the delivery of heparin subcutaneously. Due to its size and negative charge, heparin has very oral low bioavailability, this means current treatment relies on IV or SC injections [185]. PLGA nanoparticles have been investigated to create a sustained release formulation. The formulation was able to provide a SC steady release in vivo in rats over a 7 day period compared to that of 3 hours by normal SC heparin [186].

Nanoparticles are considered ideal carriers as the have a high surface to volume ratio, high capacity for drug loading [187] and can be easily modified to target specific areas on the intestinal mucosa [188]. They can also increase residence time in the GI tract due to mucoadhesion [183], increase intestinal permeability and improve solubility and dissolution rate [189].

Newer methods are being employed to increase the effectiveness of uptake by utilising the body's natural uptake mechanisms. Ligands used for uptake include; vit- 
amin $\mathrm{B}_{12}$, biotin and folate [190]. Researchers have used folate coupled chitosan nanoparticles to further increase the uptake of peptides [191]. Folic acid is actively absorbed in the GI tract by a proton-coupled FA transporter. This transporter would also allow for folate coated nanoparticles to be actively absorbed in the GI tract. The folate linked chitosan nanoparticles were studied for delivery in both in vitro and in vivo systems. in vitro studies showed a statistically significant improvement in insulin delivery using folate linked nanoparticles, compared to free insulin and chitosan nanoparticles. When these formulations were given to diabetic rats orally, the folate linked nanoparticles resulted in a more sustained decrease in blood glucose compared to compared to free insulin and chitosan nanoparticles [191].

A nanoparticle insulin delivery system developed by Oshadi (Israel) has reached Phase II clinical trials. Pharmacologically inert silica nanoparticles with an adsorbed polysaccharide formulated inside of enteric capsules are used [145]. Two phase I studies to assess the safety and efficacy of the system have been performed (NCT01120912 and NCT01772251), however no data has been released for either study. The product has moved to a phase II clinical trial to test the efficacy and safety of multiple doses (NCT01973920).

Another company Shanghai Biolaxy (China) uses calcium phosphate nanoparticles, for an oral insulin product. Phase I clinical study was performed on 12 healthy volunteers, either given the oral product or a SC injection of slow acting insulin. The oral product was well tolerated and produced a uniform blood glucose effect that was seen for $6 \mathrm{hrs}$. The data showed the plasma glucose reduction in both the oral or SC insulin groups, however high variability was seen in the AUC. The time of onset for either oral or SC insulin was similar [192].

\subsection{Lipid Systems}

\section{Liposomes}

Liposomes are another heavily studied carrier system; they receive great attention due to their composition [193]. Liposomes are bilayered vesicles in which an aqueous 
volume is enclosed inside of a lipid bilayer made up of natural or synthetic phospholipids [187]. They can vary in size from $10 \mathrm{~nm}$ to $10 \mu \mathrm{m}$ and can be defined as small unilamellar vesicles $(10-100 \mathrm{~nm})$, large unilamellar $(100-300 \mathrm{~nm})$ or large multi lamellar vesicles (100-3000 nm) [7]. Hydrophilic peptides maybe encapsulated in the inner core and hydrophobic peptides in the lipid bilayer [194].

Liposomes have been used by Cui et al to deliver insulin orally using dipalmitoylphosphatidylglycerol based liposomes. A number of different liposome formulations were prepared using different sterols and tested for stability and hypoglycaemic effects in rats. The liposomes containing ergosterol has the greatest ability to protect insulin against degradation in the GI stability studies. Although all the sterol based liposomes resulted in a hypoglycaemic effect, this was not greater than of that of SC insulin. When the ergosterol based liposomes were injected SC they produced the same extent of reduction in blood glucose as SC insulin. This results showed that an improvement in GI stability of the liposomes was required [195].

Liposome's limitations are their fast elimination from the blood and GI tract [196]. However, methods have been developed to overcome this limitation. To help overcome this a number of strategies have been applied, such as adsorption of carrier groups to allow a better targeted liposome and the formation of stealth liposomes. Stealth liposomes are created by coating liposomes with PEG so they cannot be detected by the mononuclear phagocyte system (MPS) [193]. Diasome Pharmaceuticals (USA) have developed a phospholipid bilayer system called hepatic-directed vesicle-insulin (HDV-1) for the of diabetic mellitus [197]. A preclinical study in diabetic dogs showed efficient delivery of insulin to liver showed a decrease in blood glucose after 1 hour. The authors also showed that HDV-1 is more effective than SC inuslin in preventing secondary hypoglycaemia as a side effect of over delivery of insulin [197]. A phase II study (NCT02794155) in 150 patients over 26 weeks has been planned, however no Phase I study data could be found on the Clinical Trials database.

SC delivery of interleukin-7 (IL-7) was also investigated using liposomes. The liposomes were 1-5 $\mu \mathrm{m}$ in size and helped to improves its half life by 26 fold, with a similar bioavailability to that of IV IL-7. Due to the size of the liposomes there was an increased residence time at the site of injection of 7.9 days [198]. 


\section{Solid Lipid Particles}

Solid lipid nanoparticles (SLN) are spherical particles made from lipids or lipid mixtures that are solid at body temperature with a diameter from 50 to $1000 \mathrm{~nm}$. The production is normally based on double emulsion techniques with water in oil in water emulsions. The lipids involved are often made from stearate, palmitate and their derivates such as a PEG stearate [187].

The delivery of oral insulin was enhanced using lectin modified solid lipid particles. When tested for gastric stability, SLNs were significantly more stable than unencapsulated insulin. When these preparations were delivered orally the profile of blood glucose reduction was similar to that of SC insulin. The lowest level of blood glucose was reached within 60 minutes, although the overall bioavailability was less than $10 \%$ [199]. 


\subsection{Aims and Objectives}

\section{Aims}

The work of this project is based on a peptide with a similar structure to OXT that has emerged from an internal drug discovery programme. Peptides suffer from very poor stability in the GI tract [7] and short circulation times [80]. Chemical modifications using fatty acid chains have been shown to help increase stability in the GI tract and increase circulation time. In previous studies attaching a palmitoyl chain to a lysine residue of insulin has shown to increase circulation time and provide a more sustained decrease in blood glucose in IV, IM and SC injections [200, 201, 202, 203, 200]. In another study using a similar approach, a prodrug of LENK was created. The prodrug consisted of a palmitoyl chain attached to the tyrosine residue. The palmitoyl could be cleaved by esterase enzymes within the body thereby regenerating the active peptide [139]. This prodrug approach also resulted in increased circulation times and increased anti-nociceptive effects both orally and intravenously [139]. OXT does not contain any lysine amino acids. Palmitoyl lysine has been conjugated to the carboxy terminus of the peptide as shown in figure 1.4 The modification is highlighted in red.

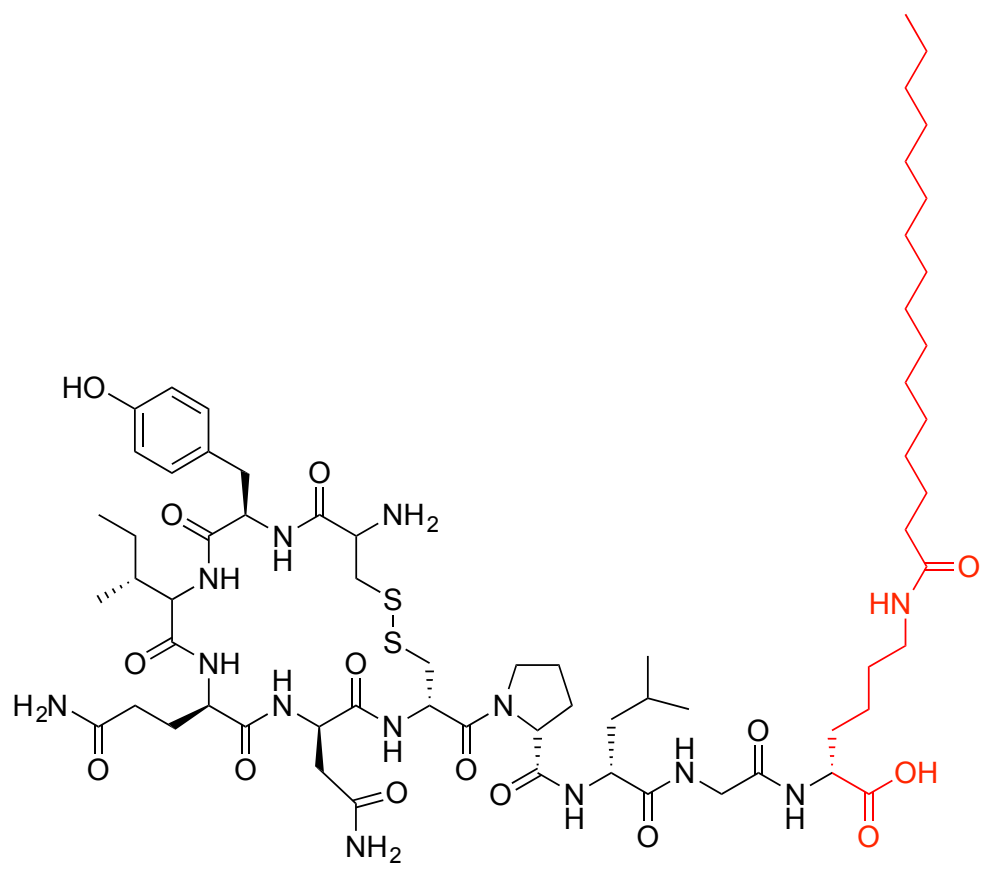

Figure 1.4: Structure of METx; red shows modification 


\section{Objectives:}

1. Characterise properties of METx-Chapter 2

2. Determine In vitro activity of METx-Chapter 3

3. Formulation development of METx-Chapter 4

4. Determine in vivo efficacy of METx formulation-Chapter 5 


\section{Chapter 2: Peptide Characterisation}

\subsection{Introduction}

The first physio-chemical studies performed on either lead or candidate molecules are known as preformulation studies. Preformulation studies provide initial foundational knowledge for formulation development [204] . Preformulation experiments can cover a range of areas, experiments conducted are used to study the lipophilicity, ionisation, solubility, stability and molecular properties of the molecule [205].

Studying the physiochemical characteristics of lead molecules provides valuable information for different teams. For chemists it will provide information to improve the chemical structure to aid solubility, permeability and stability. For biologists the same parameters would provide greater knowledge to help decide the length of drug treatment for in-vivo pharmacokinetic and pharmacodynamics studies [206].

The aim of the experiments discussed in this chapter will focus on understanding the properties of METx. Mass spectrometry (MS) was performed to confirm the structure of the peptide, the pyrene assay was performed to determine the critical micelle concentration (CMC) of METx and solubility studies were performed to determine the solubility of METx in different solvents.

\subsubsection{Mass Spectrometry}

MS uses the mass to charge ratio $(\mathrm{m} / \mathrm{z})$ of ions to help identify molecules in a simple or complex mixture by using their molecular weights [207]. The overall technique is 
based on the ability to produce ions of the molecules using a suitable method. The molecules thereby become either positively or negatively charged. These ions then pass through a mass analyser which separates these ions according to their $\mathrm{m} / \mathrm{z}$ and are reported by their relative abundance [208].

The most important step in MS is the process that converts molecules of interest into gas phase ions. This process is known as ionisation [209]. There are two MS main techniques; electrospray ionisation (ESI) [210] and Matrix Assisted Laser Desorption/Ionization (MALDI) [211].

MS by ESI can be divided into three steps; nebulisation of sample to form charged droplets, generation of ions from the droplets and transportation of droplets through the mass analyser from an atmospheric pressured environment to that under vacuum [212]. Ions formed using ESI are at atmospheric pressure; a solution containing the molecules of interest are passed through a narrow capillary, with a diameter of less than $250 \mu \mathrm{m}$. A high electric charge pulls positive charged ions to the front of the liquid. When the electrostatic repulsion becomes stronger than the surface tension, a charged droplet leaves and travels to the electrode in the gaseous environment [213]. The charged droplets are made up of both solvent and analyte, but as they pass through the mass spectrometer the solvent evaporates and the ions are analysed by the mass spectrometer [209].

MALDI acquires MS data using a different approach; the sample is co-crystallised within a solid matrix. The matrix is normally a weak acid, that absorbs UV light at the same wavelength of light emitted by the laser. The matrix has an important function, it indirectly causes the analyte to be vaporised and works as a proton donor thereby ionising the sample. The ions are then analysed using a mass analyser [214].

There are many types of mass analysers magnetic (B)/electric (E) sector mass analyser, linear quadrupole ion trap (LIT), three dimensional quadrupole ion trap (QIT), linear time of flight (TOF), TOF reflectron and Fourier transform mass analyser. The theory of mass analysers is based on two laws of physics Lorentz force law and Newton's second law of motion [215]. The basic principle is based on accelerating ions to the detector with the same energy. As the different ions are applied the same 
amount of energy due to their different masses, they reach the detector at different rates. The smaller ions reach the detector first and the larger ions reach the detector later due to their larger mass. The mass/charge ratio is calculated from the time of flight to the detector [216].

Tandem mass spectrometry (MS/MS) also provides vital secondary information. MS/MS has two stages of MS, where by the ions of interest, known as the precursor ions undergo another cycle of MS in which either the mass (m) or charge will change (z) [209]. MSMS allows more structural information to be collected about the ions of interest. The second cycle of MS generates fragments from the molecule of interest. The $\mathrm{m} / \mathrm{z}$ of these smaller fragments can be pieced together to form the precursor ion and thereby the structure and sequence of the molecule can be deduced [217].

\subsubsection{Transmission electron microscopy (TEM)}

TEM is a microscopy technique that utilises a beam of electrons to examine objects that are too small to be seen by a naked eye [218]. TEM allows for the examination of internal and external features of extremely thin samples. TEM has a similar set up to optical microscopy, except the light source is electrons [219]. The wavelength of an electron beam is around $0.0005 \mathrm{~nm}$, which is much shorter than that of optical light allowing the resolution of TEM to be a thousand times better than that of optical microscopy. The minimum size that can be seen by TEM is $0.5 \mathrm{~nm}$ [220].

A beam of electrons is generated by heating a tungsten filament and applying a high negative potential. The high negative voltage repels the freed electrons and forces them at very fast speeds towards the specimen. The electrons are reflected off samples generating a two-dimensional image, which is of high resolution and detail [221].

The samples are prepared using a negative stain to enhance the contrast of the sample. Heavy metals such as lead citrate, uranyl acetate or phosphotungstic acid are used as stains [218, 221]. 


\subsubsection{Critical Micelle Concentration}

The CMC of a molecule, generally a surfactant, is the concentration at which the surfactant starts to aggregate and form micelles, after which additional surfactant added to the system will continue to form further micelles. The most common methods to quantify the CMC are tensiometry [222] conductometry [223], calorimetry [224] and spectroscopy [225]. Each of these methods calculate the CMC as a result of the change in the physical environment at the CMC. This is achieved by titrating from a concentration of monomeric to that of micellar concentration [226].

In tensiometry the surface tension of different concentrations of surfactant are measured [227]. The surface tension of a surfactant solution decreases as the concentration of surfactant increases within the system. The concentration which the surface tension no longer decreases, is the CMC and the surface tension does not decrease anymore due to the surfactant solubility limit at the interfacial layer [228, 229].

CMC may also be measured using conductivity, but only for ionic surfactants [230]. At concentrations below the CMC the surfactants are in their monomer from so the conductivity is measured is due to the concentration of surfactant. As the concentration of surfactant rises the conductivity increases with the same linearity. As the concentration of surfactant increases above CMC the increase conductivity increases with a less steeper gradient. This concentration at which the gradient changes to become more steep is determined to be the CMC [231]. The change in conductivity at concentrations below the $\mathrm{CMC}$ is because the surfactant behaves as a strong electrolyte while above the CMC the micelles are only partially ionised, due to different degrees of surfactant ionisation [232].

Calorimetry may be used to measure the CMC. Enthalpy change is measured while titrating the surfactant solution, with the concentration above the CMC, into a surfactant free solvent (pure solvent) environment. From the enthalpy change during the titration the CMC can be calculated [233].

Determining the CMC using spectroscopy involves the use of dyes or fluorescent probes. The probes work by sensing the change of the environment from when the 
monomer converts to the micellar state. The change of environment affects the absorption and emission spectroscopy of the probe. In the monomeric stages the probe is within the aqueous environment, once micellar concentrations have been reached the probe partitions itself between the aqueous and hydrophobic core [234]. The most commonly used probe is pyrene, some others are 8-anilino-1-naphthalenesulfonic acid (ANS), Coumarin 480 (C-480), Rhodamine 6G (Rh6G) and pyrene-3-carboxaldehyde [235, 236]. A study by conducted by Dominguez et al. found the use of pyrene as a probe was the best method to measure CMC. The CMC of sodium dodecyl sulfate was measured using conductivity, a UV probe (benzoylacetone) and a fluorescence probe (pyrene). Of the three pyrene was found to be the best as it was the least influenced by experimental factors as well as giving the most precise results [232].

\subsubsection{HPLC}

High performance liquid chromatography (HPLC) is one of the most commonly used analytical tools used throughout all stages of drug discovery, development and production [237]. Liquid chromatography is a separation technique. A sample solution is injected into a column of amorphous material (stationary phase) and a liquid (mobile phase) is pumped through the column. The separation takes place due to the different rates of passage of compounds though the column; resulting in the compounds being eluted at different times. The elution is analysed by UV absorbance at a given wavelength. The technique was first developed by botanist Mikhali Tsvet after which there has been extensive use, research and development of liquid chromatography [238].

For biological samples where the samples are very complex and contain a number of different compounds the reverse phase HPLC (RP-HPLC) is very useful. The reverse phase utilises a non-polar (hydrophobic) stationary phase and an aqueous mobile phase is pumped through [239].

In analytic HPLC, the aim is to achieve a good degree of separation in order to obtain quantitative and qualitative information of the compounds of interest from a sample [240]. A complex sample may contain a wide range of components with vary- 
Table 2.1: Definition of solubility from BP 2016 [245].

\begin{tabular}{cc}
\hline Descriptive Term & $\begin{array}{c}\text { Approximate volume of solvent (ml) } \\
\text { per gram of solute }\end{array}$ \\
\hline very soluble & less than 1 \\
freely soluble from & 1 to 10 \\
soluble from & 10 to 30 \\
sparingly soluble & from 30 to 100 \\
slightly soluble from & 100 to 1000 \\
very slightly soluble & from 1000 to 10000 \\
practically insoluble & more than 10000 \\
\hline
\end{tabular}

ing degrees of solubility. Each different molecule experiences different levels of interactions with the stationary phase; this will result in different elution times. This allows the samples to be assessed for individual components [241]. HPLC is used in a range of pharmaceutical applications such as analysis of pharmaceutical drugs, for drug monitoring and for quality assurance [242].

\subsubsection{Solubility}

The most common reason for low bioavailability after administration is due to poor solubility and low permeability [132]. The problems faced due to solubility provide development problems for both invasive and non-invasive delivery systems [243]. The problem of solubility is becoming more of a common challenge with 10-30\% of marketed drugs and $60-70 \%$ of developmental stage compounds having solubility problems [244].

The USP (US pharmacopeia), BP (British pharmacopeia) and EUP (European pharmacopeia) categorise solubility in terms of quantity, the volume of solvent (ml) required to dissolve $1 \mathrm{~g}$ of solute. These categories are shown in table 2.1.

Solubility is the extent to which a solute; a solid, liquid or gas is able to dissolve in a solvent; a solid liquid or gas to from a homogenous solution. The solubility refers to the amount of solute that passes into solution when an equilibrium is reached between the solute in solution and undissolved solute [246]. IUPAC (International Union of 
Pure and Applied Chemistry) defines solubility as "the analytical composition of a saturated solution, expressed in terms of the proportion of a designated solute in a designated solvent, is the solubility of that solute. The solubility may be expressed as a concentration, molality, mole fraction, mole ratio, etc" [247]. The solvent is normally a liquid or mixture of two liquids [132]. As a general rule "like dissolves like", essentially the structure and polar nature of the molecules determines the solubility. Non-polar solutes dissolve in non-polar solvents and vice-versa polar solutes in polar solvents [248]. As a molecule becomes more hydrophobic there is a reduction in dipole moments, polarity of molecules, and inter-molecuar forces which turns it from being hydrophilic to hydrophobic. Due to favourable dipole-dipole attractions between solvent molecules and solute molecules, polar liquids tend to dissolve readily in polar solvents. Thereby polar molecules, especially those that can form hydrogen bonds with water are more soluble in water [249].

This is shown the example of hydrocarbons, methanol is able to dissolve in water (polar solvent), but is insoluble in hexane (non-polar solvent). As the length of the hydrocarbon chain increases the molecules become less soluble in water and more soluble in hexane, for example propanol is not soluble in water but soluble in hexane [250]. This is because the alcohol group of methanol forms hydrogen bonds with water. As the number of carbons increases, the alcohol group and hydrogen bonding becomes a smaller part of the molecule and the overall molecule becomes more hydrophobic thereby having less solubility in water [249]. 


\subsection{Materials \& Method}

\subsubsection{Materials}

Table 2.2: Materials used for physiochemical studies of METx

\begin{tabular}{ll}
\hline Materials & Supplier \\
\hline Absolute Ethanol & Fisher \\
Acetonitrile & Sigma \\
Methanol & Sigma \\
METx & Severn Biotech \\
Pyrene & Sigma \\
Water & Millipore \\
\hline
\end{tabular}




\subsubsection{Methods}

\subsubsection{Mass Spectrometry}

Electrospray ionization (positive) mass spectrometry was performed by UCL School of Pharmacy Structural Chemistry Services. High resolution accurate mass measurement was done using a Waters Q-TOF premier Tandem Mass Spectrometer in V mode positive. METx was dissolved in acetonitrile $(\mathrm{MeCN})$ : water $(1: 1)$ with formic acid $(0.1 \%)$.

\subsubsection{TEM}

METx was prepared for TEM by either suspending METx in water or by probe sonication of METx in water. METx was sonicated on ice for 15 minutes, with a cycle of 5 minutes on and 3 minutes off (Q Sonica, Cole Parmer Instruments Co. Ltd, UK). The Q Sonica was used with a microtip and set to $25 \%$ of its maximum output. TEM was performed by placement of a drop of the sample on a Formvar/carbon coated grid. Excess sample was blotted off with Whatman No. 1 filter paper and the sample was negatively stained with uranyl acetate $(1 \%$ w/v). Samples were imaged using a CM120 BioTwin Transmission Electron Microscope (Philips, USA). Images were captured using an AMT digital camera (Woburn, USA).

\subsubsection{Determination of CMC}

The CMC of METx was determined using the pyrene assay. The pyrene assay was performed as previously described [182, 251] . An aqueous solution of pyrene $(2 \mu \mathrm{M})$ was prepared by dissolving pyrene in absolute ethanol $\left(0.4 \mathrm{mg} \mathrm{ml}^{-1}\right)$. Under a stream of nitrogen the pyrene solution $(100 \mu \mathrm{l})$ was dried in $100 \mathrm{ml}$ volumetric flask. The solution was made up to $100 \mathrm{ml}$ using MilliQ water. Using this pyrene solution as the solvent various concentrations of METx solutions were prepared $\left(200 \mu \mathrm{g} \mathrm{ml}^{-1}\right.$ to $2 \mathrm{pg}$ $\mathrm{ml}^{-1}, 1.45 \times 10^{-10}$ to $\left.1.45 \times 10^{-4} \mathrm{M}\right)$. Each solution was probe sonicated on ice for 15 minutes with a cycle of 5 minutes on and 3 minutes off with (Q Sonica, Cole Parmer 
Instruments Co. Ltd, UK). The Q Sonica was used with a microtip and set to $25 \%$ of its maximum output. The fluorescence spectra was recorded using a spectrophotometer (LS 50B Fluorescence Spectrometer, Perkin Elmer, Massachusetts, United States). The excitation was set at $335 \mathrm{~nm}$ and the emission was measured from 350 to 500 $\mathrm{nm}$. The ratios of the intensity of the third $(383 \mathrm{~nm})$ and first $(375 \mathrm{~nm})$ peaks in the emission spectra (the I3/I1 ratio) were recorded and the critical micellar concentration was calculated at the concentration at which the rapid change in gradient is observed. Each concentration was repeated in triplicate.

\subsubsection{HPLC Method}

HPLC was performed to analyse the content of peptide within the given solutions. HPLC was performed using an Agilent 1200 HPLC system (Agilent Technologies, UK) equipped with a quaternary pump, degasser, auto sampler and a UV detector. Samples were chromatographed over a reverse phase column: Phenomenex Onyx Monolithic C18 column (5 $\mu \mathrm{m}, 4.6 \times 100 \mathrm{~mm})$ connected in series with a guard column (5 $\mu \mathrm{m}, 4.6 \times 10 \mathrm{~mm})$ (Phenomenex, UK). The column temperature was kept constant at $35^{\circ} \mathrm{C}$. Separation was performed in a gradient method of $20-95 \%$ MeCN over 15 minutes, the gradient is shown in table 2.3 below. The VWD detector was set at 280 $\mathrm{nm}$. The chromatograms were obtained and analysed using HPLC Chemstation (version 01.05 for windows) software.

Table 2.3: HPLC solvent gradient for METx.

\begin{tabular}{l|ccccccc} 
Time (minute) & 0 & 2 & 15 & 17 & 19 & 20 & 25 \\
\hline MeCN $(\%)$ & 20 & 20 & 80 & 95 & 95 & 20 & 20 \\
\hline Flow rate $\left(\mathrm{ml} \mathrm{min}^{-1}\right)$ & 1.5 & & & & & &
\end{tabular}

\subsubsection{Solubility Studies}

Solubility of METx was determined using the shake flask method. An excess of METx (1mg) was suspended in MilliQ water, phosphate buffered saline (PBS), methanol 
$(\mathrm{MeOH})$, dimethylformamide (DMF) and $\mathrm{MeCN}(1 \mathrm{ml})$ and left to shake for 24 hours at room temperature. Dissolved METx and undissolved METx was separated by centrifugation for 10 minutes at 3,000 rpm (MSE micro centaur, MSE, UK). The supernatant for $\mathrm{MeOH}$ and DMF was diluted four fold using $\mathrm{MeOH}$ and DMF respectively. This was done as the solubility of METx in MeOH and DMF was outside of the HPLC calibration curve's quantification limits. The supernatant of each solvent was analysed using HPLC as described in section 2.3. Each solvent was repeated in triplicate. 


\subsection{Results}

\subsubsection{Mass Spectrometry}

METx was dissolved in water: MeCN with formic acid $(0.1 \%)$ and analysed by ESI and tandem MSMS. The mass of METx (expected mass, in Daltons) is 1374.75. The product ion peak correlates well with the molecular ion of METx producing a parent ion peak at 1374.75 amu. Tandem MSMS was used identify the correct sequence of the METx. Tandem MSMS was performed multiple times to achieve all the fragments, however it did not result in fragmentation of the cyclic ring of METx. The $y$ and $b$ fragments were produced for the four amino acid peptide tail. The same fragmentation was found with oxytocin in the literature, in which the cyclic disulphide ring remains intact [252]. The sites of fragmentation and the table of peptide fragments for METx are shown in figure 2.1 and 2.2 and table 2.4.

Table 2.4: Identified MSMS fragments of METx.

\begin{tabular}{ccccc}
\hline $\mathrm{b}$ fragment & $\mathrm{m} / \mathrm{z}$ observed & Residue y & fragment & $\mathrm{m} / \mathrm{z}$ observed \\
\hline 104.02 & & $\mathrm{C}$ & 1270.74 & \\
267.08 & & $\mathrm{Y}$ & 1107.67 & \\
380.16 & & $\mathrm{I}$ & 994.59 & \\
508.22 & & $\mathrm{Q}$ & 866.53 & \\
622.26 & & $\mathrm{~N}$ & 752.49 & \\
723.27 & $723.26(\mathrm{~b} 6)$ & $\mathrm{C}$ & 650.27 & $652.51(\mathrm{y} 4)$ \\
821.32 & $803.31\left(\mathrm{~b} 7-\mathrm{H}_{2} \mathrm{O}\right)$ & $\mathrm{P}$ & 553.22 & $555.46(\mathrm{y} 3)$ \\
933.41 & $933.42(\mathrm{~b} 8)$ & $\mathrm{L}$ & 440.13 & $442.37(\mathrm{y} 2)$ \\
990.43 & $990.42(\mathrm{~b} 9)$ & $\mathrm{G}$ & 383.11 & $385.33(\mathrm{y} 1)$ \\
\cline { 2 - 4 } 1374.37 & 1374.76 & $\mathrm{~K}-(\mathrm{palm})$ & & \\
\hline \multicolumn{5}{c}{}
\end{tabular}



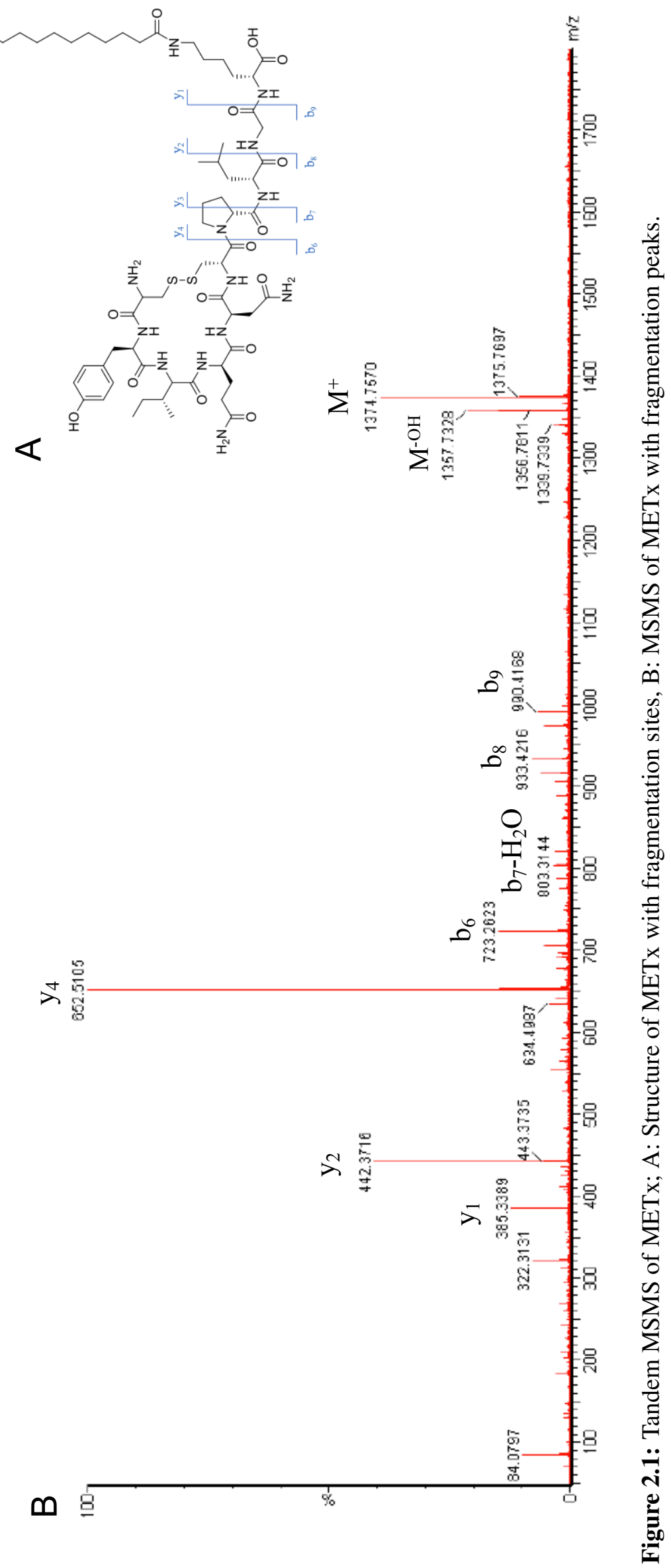


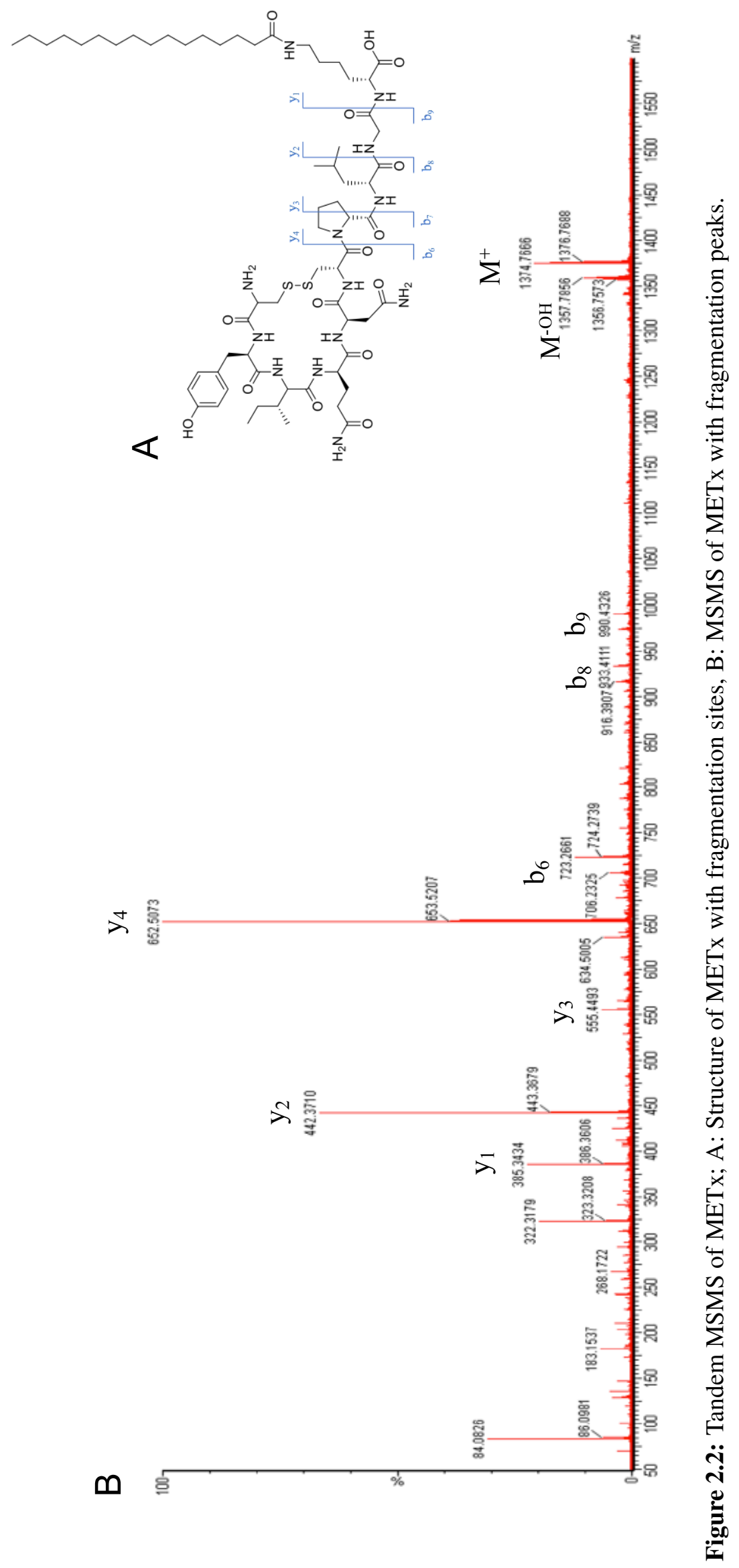




\subsubsection{TEM}

TEM images of METx dispersed in water show the formation of fibrous structures, around $100 \mathrm{~nm}$ in length and 10-15 nm wide as seen in figure 2.3 A and B. Sonication of METx on ice for 15 minutes results in a decrease in the presence of the fibrous structures and the formation of spherical aggregations. This can be seen in figure 2.3 C and D. In figure 2.3 D METx aggregates are seen with a size between 10-50 nm.
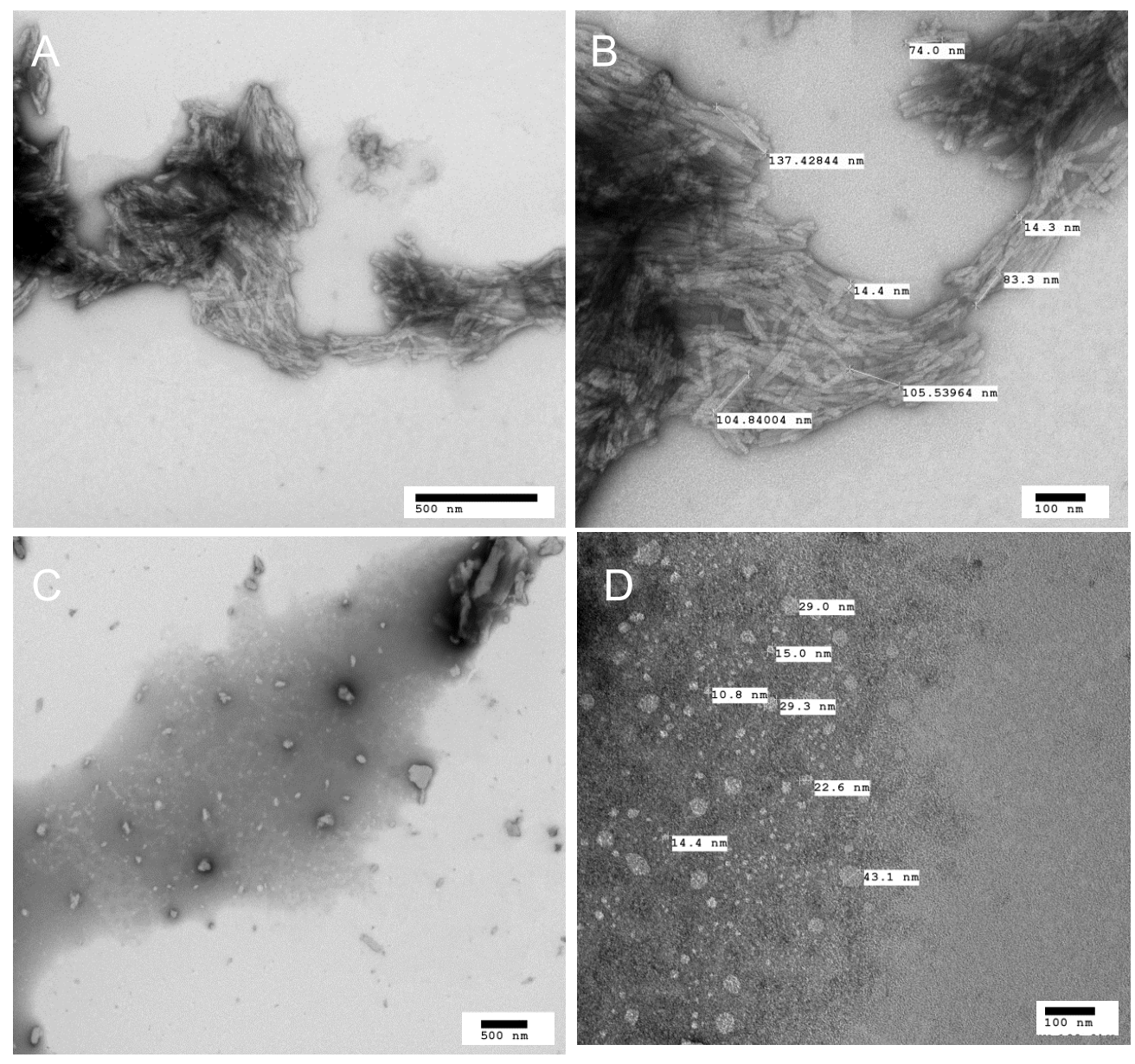

Figure 2.3: TEM pictures of METx suspended (A and $B$ ) and sonication $(C$ and $D)$ in water at $1 \mathrm{mg} \mathrm{ml}^{-1}$.

\subsubsection{Pyrene Assay}

The pyrene assay uses a hydrophobic probe that exhibits different emission spectra depending on its environment. Below the CMC, pyrene is in a greater polar environment which means pyrene cannot be encapsulated resulting in a greater intensity of 
peak 1 at $375 \mathrm{~nm}$. In environments above the CMC there is a greater hydrophobic environment allowing pyrene to be held within the hydrophobic core of micelles this leads to a greater intensity of peak 3 at $383 \mathrm{~nm}$. The pyrene assay uses the ratio of peak intensities of the first and third peak of various concentrations of compound to calculated the CMC

Using the pyrene assay as described above, the i3/i1 intensities were measured for METx from $2 \mathrm{pg} \mathrm{ml}^{-1}\left(1.45 \times 10^{-10} \mathrm{M}\right)$ to $200 \mu \mathrm{g} \mathrm{ml}^{-1}\left(1.45 \times 10^{-4} \mathrm{M}\right)$ by stepwise dilution, shown in figure 2.4. The CMC of METx was calculated to be $27.4 \mathrm{mg} \mathrm{L}^{-1}$ $\left(2 \times 10^{-5} \mathrm{M}\right)$.

\section{CMC of METx in Pyrene Assay}

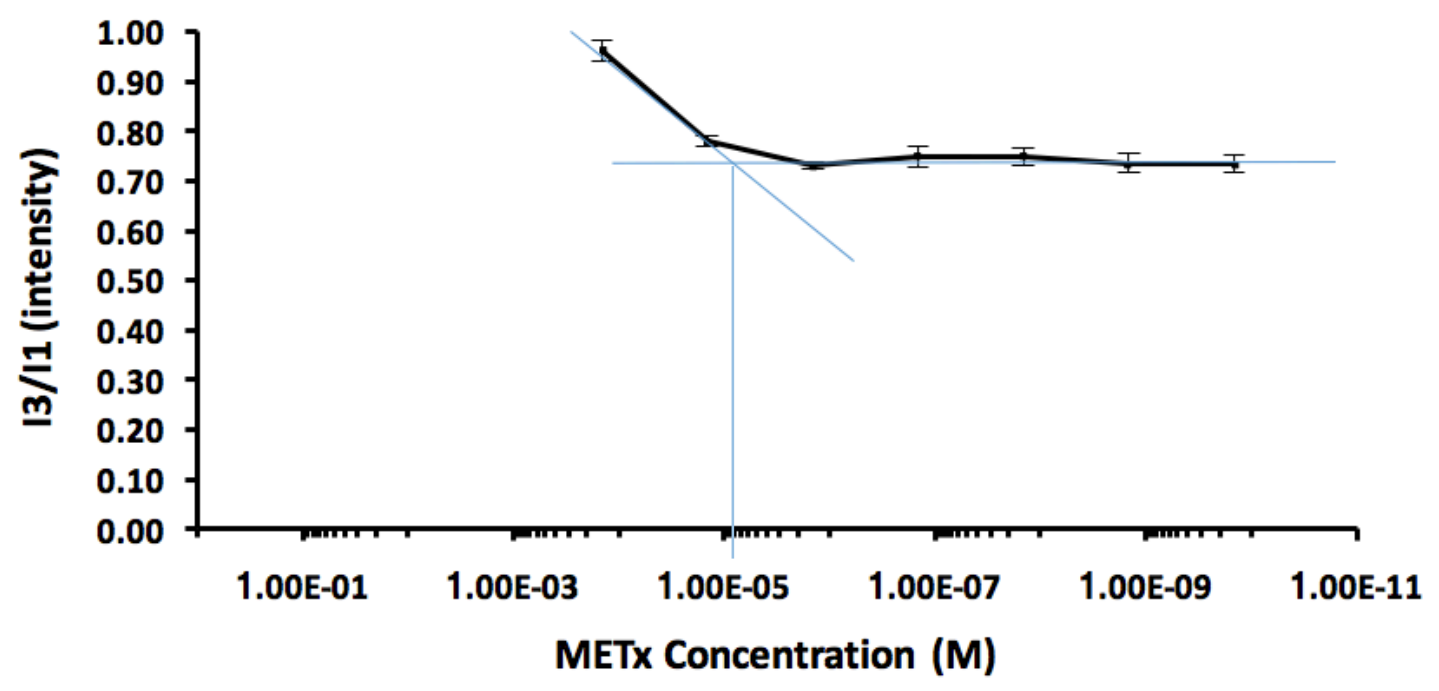

Figure 2.4: Determination of CMC of METx using pyrene assay.

\subsubsection{HPLC}

A calibration curve was created for METx using HPLC. METx (1 mg) was dissolved in DMSO (dimethyl sulfoxide, $1 \mathrm{ml}$ ) and diluted step wise with DMF, shown in figure 
2.5. The liner calibration was $y=0.34 x+2.8\left(r^{2}=0.9982\right)$ with the limit of detection of $1 \mu \mathrm{g} \mathrm{ml^{-1 }}$.

\section{METx Calibration Curve}

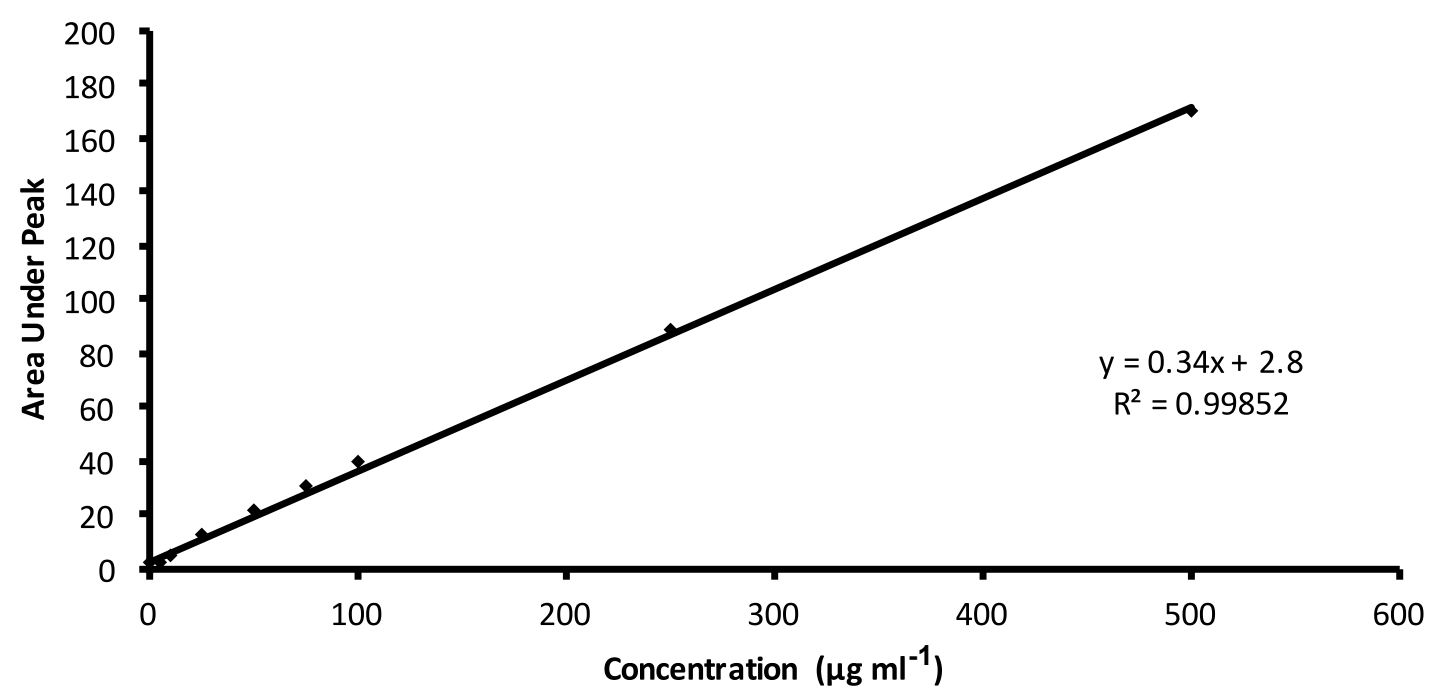

Figure 2.5: HPLC calibration curve of METx.

\subsubsection{Solubility Studies}

The solubility of METx was measured in various aqueous and organic solvents using the shake flask method. Undissolved METx was separated form solubilised METx by centrifugation.

Table 2.5: Solubility of METx in different solvents.

\begin{tabular}{cc}
\hline Solvent & Concentration $( \pm)\left(\mu \mathrm{g} \mathrm{m}^{-1}\right)$ \\
\hline Water & Undetectable \\
PBS & Undetectable \\
Acetonitrile & $19.45(5.5)$ \\
Methanol & $362.71(47.8)$ \\
DMF & $833.17(98.9)$ \\
\hline
\end{tabular}


The solubility of METx in aqueous solvents was below the limit of detection of HPLC. In water and PBS the solubility of METx was below $1 \mu \mathrm{g} \mathrm{ml}^{-1}$. A solubility of less than $1 \mu \mathrm{g} \mathrm{ml}^{-1}$ is considered to be either practically insoluble or insoluble. In organic solvents METx showed higher solubility. The solubility of METx was measured in $\mathrm{MeCN}, \mathrm{MeOH}$ and DMF. METx showed highest solubility in DMF followed by methanol and acetonitrile respectively. Table 2.5 shows the overall data for solubility studies. 


\subsection{Discussion}

Within the experiments of this chapter initial preformulation experiments were untaken to understand a few properties of METx. The results from these experiments would help plan with some aspects of future experiments. There is a large number of preformulation experiments that may be undertaken which aim to characterise the bulk material and its properties of the molecule such as solubility, stability and its isoelectric point (pI) [205].

MS and tandem MSMS were used to confirm the structure of METx. The molecular weight of METx is 1374, the product peak of METx was seen to fly at 1374 . Upon fragmentation of METx it was found that fragmentation only took place on the peptide tail and not within the cyclic disulphide ring of METx. All the fragments after the cyclic ring were identified, this helped to confirm the structure of METx. The same molecular weight fragments were found by Erve et al. [252].

TEM shows the macroscopic structure of the given sample. When METx is suspended in water without any energy applied it shows the formation of fibrous like structures, which can be seen individually upon magnification. The fibrous structures have dimension of around $100 \mathrm{~nm}$ in length and 10-15 nm wide. These structures are similar to those literature by Lalatsa et al. where the attachment of palmitoyl to LENK resulted in fibrous structures [139] and also by Weronski et al. with the attachment of palmitoyl to two different 10 amino acid sequence peptides [253]. When METx is probe sonicated it shows the formation of circular aggregations with a diameter of $10-50 \mathrm{~nm}$. TEM provided the first indication that METx aggregates. This is important as it warrants the next study to determine the critical micelle concentration of METx.

There a number of ways to measure the CMC of a given molecule, in this study pyrene was used as a probe to determine the CMC. Depending on the surrounding environment of pyrene; the transition between aqueous to lipophilic; alter the relative intensities calculated between the first and third peak. The CMC of METx has been

calculated to be $2 \times 10^{-5} \mathrm{M}\left(20 \mu \mathrm{M}, 27 \mathrm{mg} \mathrm{L}^{-1}\right)$. Similar values of CMC were found by other groups that used the palmitoyl chain either for receptor binding or increased 
permeability within their given molecules [254, 255]. The CMC of palmitoylated GALA (14 amino acid) was measured using the pyrene assay, and found to be $12 \mu \mathrm{M}$ [255]. Another study observed CMC a tripeptide conjugated with either palmitic acid $\left(\mathrm{C}_{16}\right)$ or stearate $\left(\mathrm{C}_{18}\right)$. The study found that both peptides have $\mathrm{CMC}$ values in the micro-molar range, with palmitoyl linked peptide CMC higher than that of the stearate linked peptide, 60 and $43 \mu \mathrm{M}$ respectively [256].

Another preformulation experiment was to study the solubility of METx. The solubility of METx was measured using the shake flask method, with a concentration of $1 \mathrm{mg} \mathrm{ml}^{-1}$ left to shake for 24 hours at room temperature. This concentration was considered an excess as none of the solvents produced concentrations above $1 \mathrm{mg} \mathrm{ml}^{-1}$. In water and PBS the concentration of METx was below that of the detection limit, less than $1 \mu \mathrm{g} \mathrm{ml}^{-1}$. Even if the solubility was at $1 \mu \mathrm{g} \mathrm{ml} \mathrm{m}^{-1}$, when this is scaled up to the volume required for $1 \mathrm{~g}$ it is $1,000,000 \mathrm{ml}(1000 \mathrm{~L})$. Within the criteria of the USP, BP and EUP; METx is considered practically insoluble within aqueous environments. METx is an OXT derivate, it has been modified by the attachment of palmitoyl lysine to the tri-peptide tail of OXT. OXT is considered very soluble in water and PBS, the stated value of solubility in PBS ( $\mathrm{pH} 7.2$ ) is $5 \mathrm{mg} \mathrm{ml}^{-1}$. As lysine is very soluble amino acid in water $\left(100 \mathrm{mg} \mathrm{ml}^{-1}\right)$, it can be assumed that the addition of plamitoyl that makes METx insoluble in water. However this lack of solubility is a problem that is experienced both in marketed drugs as well as those in development stages. Estimates suggest around $40 \%$ of marketed medications are classified and $90 \%$ of developmental drugs are characterised as poorly soluble [257]. METx was found to more soluble in organic solvents. METx has the highest solubility in DMF followed by $\mathrm{MeOH}$ and then $\mathrm{MeCN}$.

Similar solubility results were found in the literature for palmitoylated peptides, Gkourogianni et al. found palmitoylated peptides were insoluble in water but more soluble in organic solvents, where as the non-palmitoylated peptides were freely soluble in aqueous mediums [258, 259]. Another example of decrease in solubility on palmitoylation is example of this is liraglutide, a commerical GLP-1 agonist. Liraglutide is made up of the GLP-1 linked to palmitic acid via a glutamic acid linked [260]. GLP-1 is a 38 amino acid peptide that has a solubility of $1 \mathrm{mg} \mathrm{ml}^{-1}$, however 
liraglutide has a lower solubility with water at $\mathrm{pH} 7$ [261]. The impact of solubility by conjugation of a lipophilic group to a peptide is well-known, its reduction in solubility is due to an increase the molecule's hydrophobicity [262].

In conclusion, two properties of METx have been investigated, its ability to aggregate to form micelles and its solubility. METx was found to form spherical aggregates with a CMC of $20 \mu \mathrm{M}$. METx exhibited undetectable solubility in aqueous medium and was higher in organic solvents, with the highest solubility of METx in DMF. These results will be part of important steps in further studies, the concentration of METx tested in the in vitro studies is kept below that of MET's CMC. This is to ensure the effect of METx in its monomeric form is observed and not that of its aggregated form. Also the solubility studies are important at they will help with the best solvent for isolation of METx in the formulation stability studies. 


\section{Chapter 3: In Vitro Studies}

\subsection{Introduction}

In vitro studies have become an essential tool to study and understand cellular biological and physiological processes for more than 100 years [263]. In vitro assays provide an alternative method to some animal studies, this means the number of animal studies may be reduced. Animal studies can be slow, costly and less ethically accepted [264]. In vitro studies can be used as a tool to build an initial picture as well as play a key role in screening [265]. The results from in vitro studies can be used with confidence as these models undergo some of the most through validation in life sciences [266]. The aims of the experiments discussed in this chapter are to study the activity of METx on the $\mathrm{V}_{2} \mathrm{R}$, by measuring the production of cAMP.

\subsubsection{Vasopressin}

The $\mathrm{V}_{2} \mathrm{R}$ is found on the basolateral membrane of the collecting duct in the kidney [267]. The binding of AVP to the $\mathrm{V}_{2} \mathrm{R}$ leads to the insertion of the water channels, AQP2, into the luminal surface of the collecting tubule cells [26]. The $V_{2} R$ is from the $\beta$ subfamily of rhodopsin like GPCR [268], which uses cAMP as its secondary messenger [269].

Activation of the $V_{2} R$ by AVP stimulates the Gs-coupled protein, $G \alpha_{s}$, which activates adenyl cyclase. The activation of adenyl cyclase causes the production of cAMP from adenosine triphosphate (ATP) which in turn activates protein kinase A (PKA). 
The activation of PKA results in the phosphorylation and translocation of AQP2 within the intracellular storage vesicles to the apical plasma membrane. Making the membrane permeable to water [270]. This pathway results in exocytosis of aquaporin water channel vesicles (AQPWC) and also inhibits the endocytosis of the AQP2. This dual action ensures an increased amount of AQP2 in the membranes thereby maximising the permeability of the collecting duct [271, 272, 273]. Figure 3.1] shows an overview of the cascade that leads to the reabsorption of water from the collecting duct. The reabsorbed water is returned back to the blood, helping to decrease plasma osmolality [274].

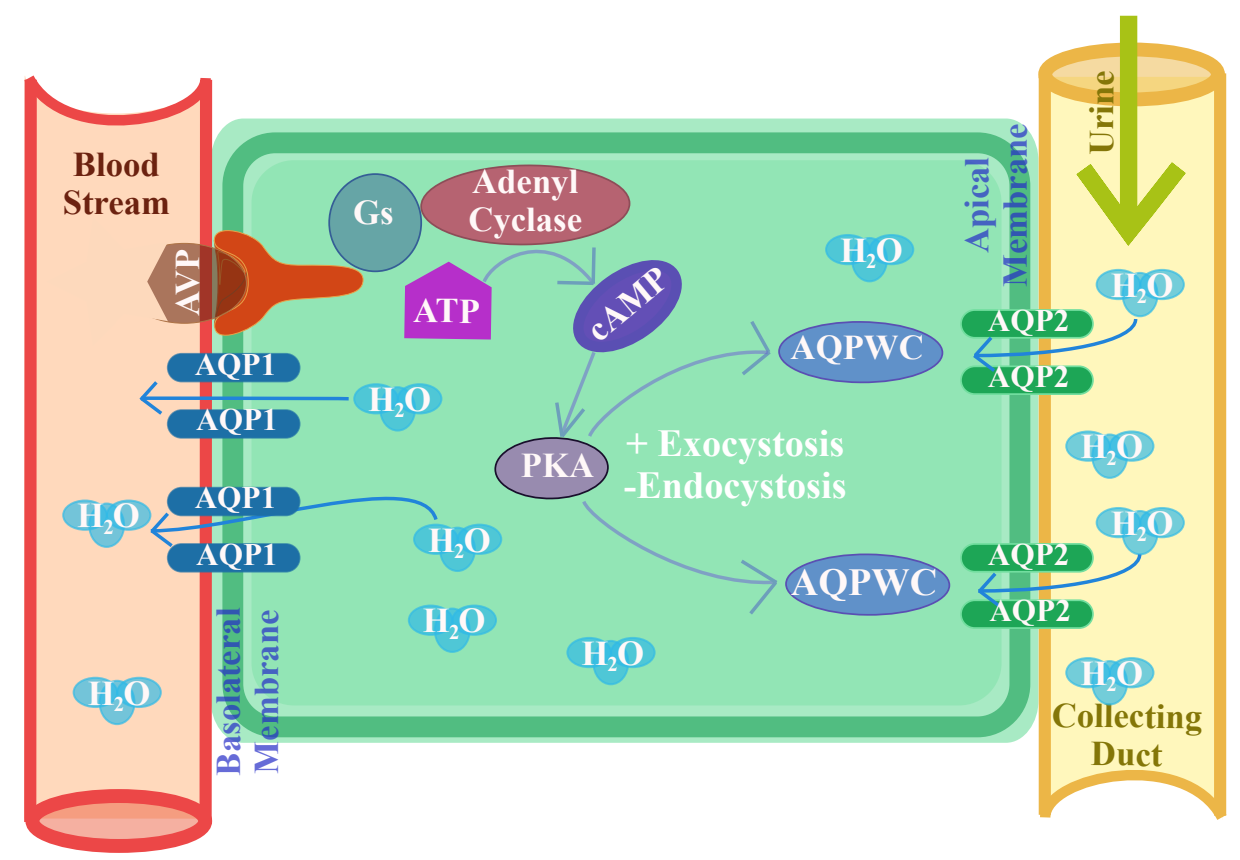

Figure 3.1: $\mathrm{V}_{2} \mathrm{R}$ cellular cascade.

cAMP is the secondary messenger within the $V_{2} R$ cascade. cAMP is derived from adenosine triphosphate (ATP) by adenyl cyclase found on the inside of plasma mem- 
branes, that is activated due to binding of an agonist. cAMP plays a critical role in cell signalling mechanisms of other hormones such as glucagon and adrenaline. cAMP causes the phosphorylation of proteins via protein kinase [275]. In the AVP; cascade PKA activated to due to cAMP activity phosphorylates at Ser-256 on the AQP2. This results in the insertion of AQP2 channels in the apical membrane, making the membrane water permeable [270].

\subsubsection{Ligand Screening Studies}

There are a number of methods to screen ligands for GPCRs. The wide range of assays used to screen for ligands of GPCR may be divided into two major groups; either receptor binding assays or functional assays. The functional assays can be further divided into two groups; G-protein dependent functional assays, or G-protein independent functional assays [276]. Irrespective of assay used there are some basic requirements that need to be met; the assay has to be pharmacologically relevant, reproducible, minimise assay costs, maximise assay quality, reduction of solvent effects within the assay. The majority of chemical libraries are stored in either ethanol or DMSO so assays need to be devised such that they are not sensitive to the concentration of solvent used [277]. The commercial availability of each group and an overview of their advantages and disadvantages is shown in table 3.1 . 


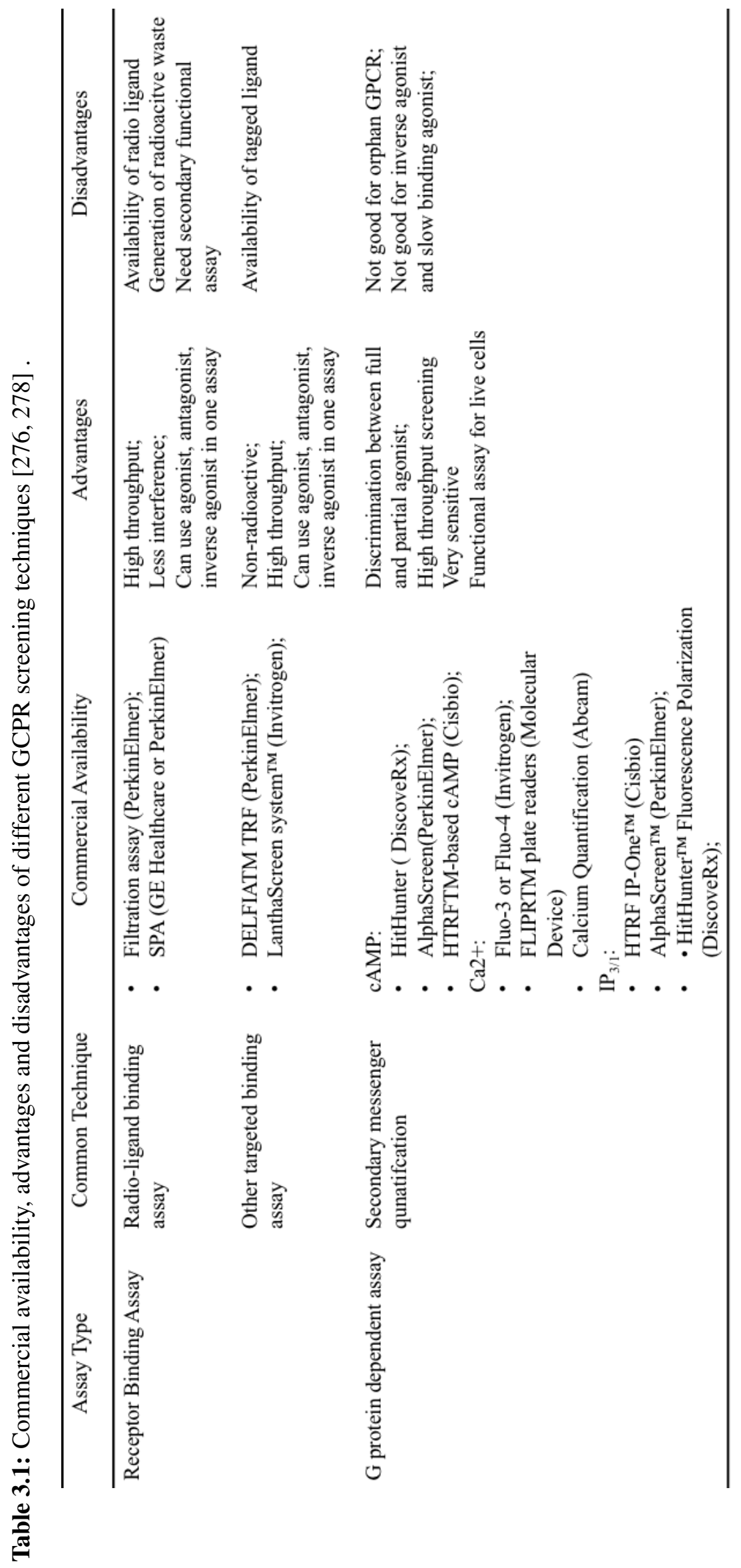




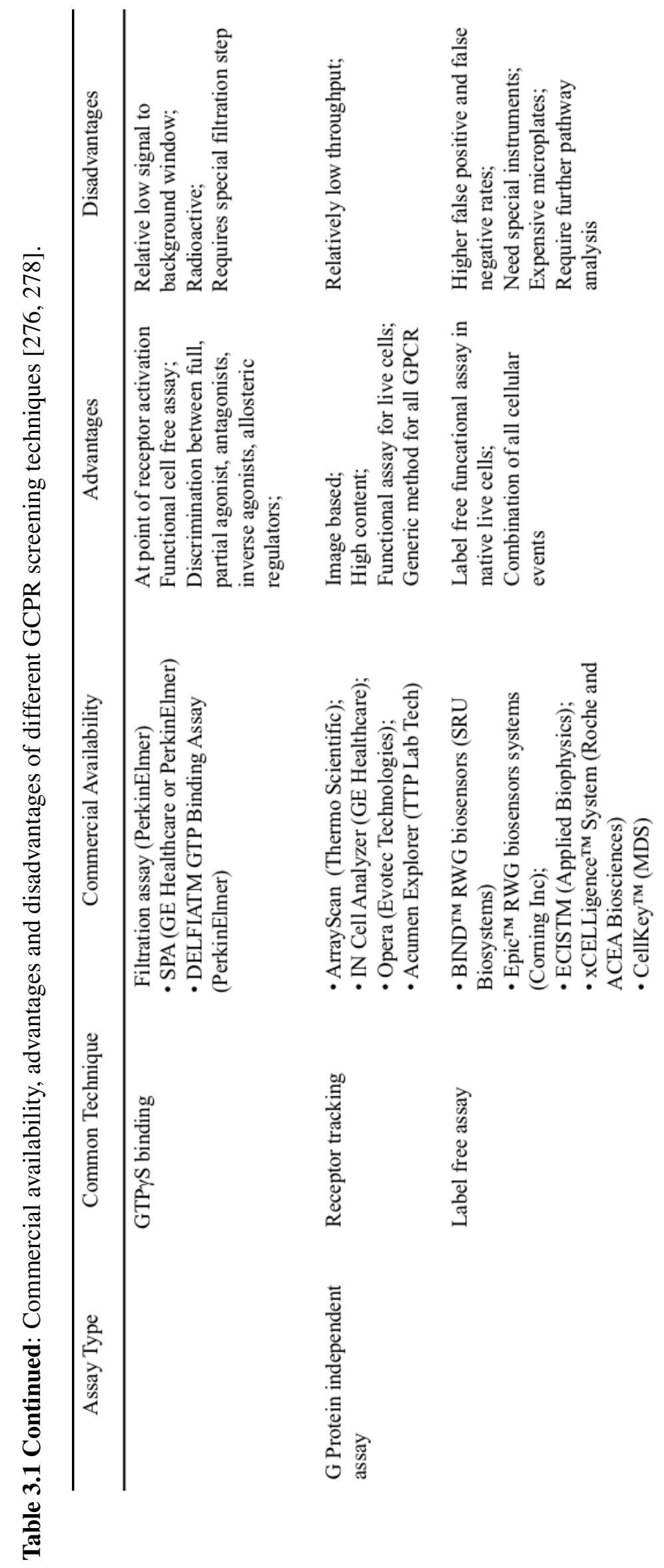




\subsubsection{Receptor Binding}

Receptor binding assays rely on the ability to measure the interactions between one or more molecules and a target site. This interaction can be either two proteins binding to one another, a molecule binding to a receptor, or a protein binding to a nucleic acid [279]. Historically, binding assays were performed using radioactive labelled ligands, but with the disadvantages related to the disposal of radioactive waste, costs, health hazards and requirements of special licences, newer technologies have been developed. Newer labels using colorimetric, fluorescence or luminescence detection methods have been developed to provide similar data, with a reduction of the risks to the investigator and environment [280]. Irrespective of the labelling technique or detection method; the overall methods remain the same. Binding assay experiments start with a preparation containing the receptor/protein of interest which is incubated with the labelled ligand of interest, after which either the bound or free ligand concentration is measured. These steps are repeated using a range of concentrations of unlabelled ligands [281].

Binding experiments can be either kinetic or equilibrium, kinetic experiments are used to define optimal assay conditions. Equilibrium experiments may further be divided into either saturation or competition experiments. In saturation experiments the binding affinity of the labelled ligand is determined. Increasing concentrations of labelled ligand are incubated with the receptor/protein from which dissociation constant $\left(\mathrm{K}_{\mathrm{d}}\right)$ may be calculated, as show in figure 3.2 [282]. $\mathrm{K}_{\mathrm{d}}$ signifies the strength of the interaction between the ligand and a given target, $\mathrm{K}_{\mathrm{d}}$ is the concentration of ligand required to bind to $50 \%$ of receptors within the system [283], as shown in figure 3.2 . Competition experiments sometimes referred to as displacement experiments can also be performed. A fixed concentration of labelled ligand competes with different concentrations of an unlabelled inhibitory ligand. This type of experiment calculates an inhibitory concentration $\left(\mathrm{IC}_{50}\right)$, which is the concentration of the inhibitor causing $50 \%$ inhibition of binding of a given substance [282]. 


\section{Saturation Binding Curve}

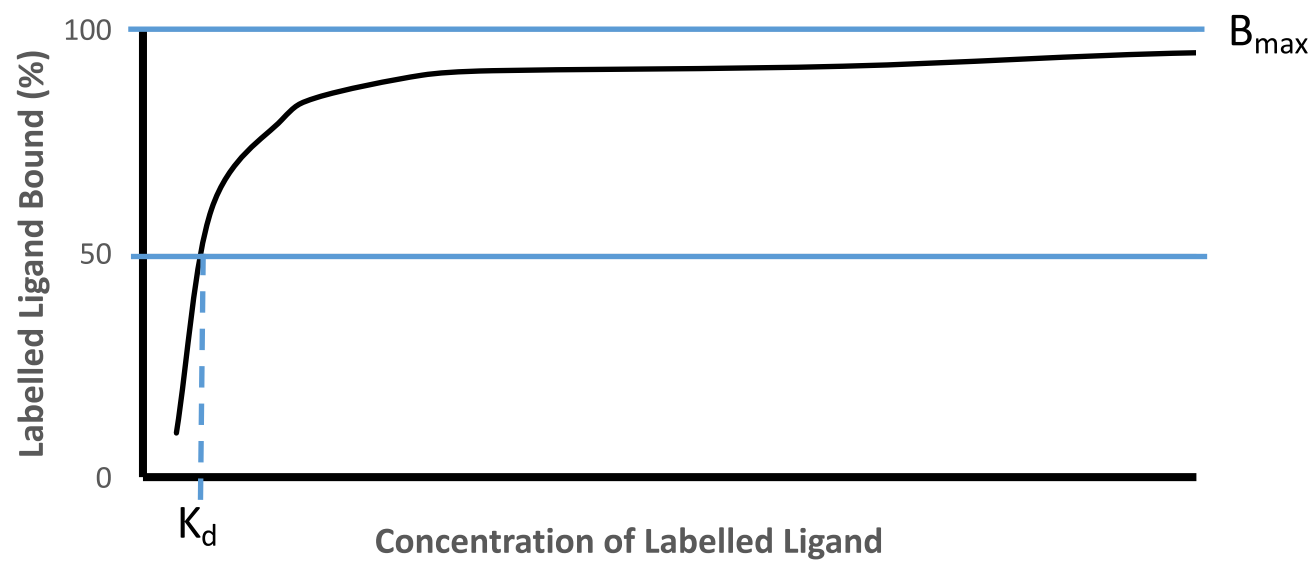

Figure 3.2: Ligand binding saturation curve.

\subsubsection{Functional Assays}

The other screening assays are functional assays, which measure activity. Functional assays either measure $\mathrm{G}$ protein activation, or the generation molecules derived due to G protein activation. This can be the production of a secondary messenger or another molecule in the activation cascade [276]. Like the receptor binding assays the set up for functional assay is similar with a living cells expressing the receptors of interest and incubated with the molecule of interest either alone or in order to understand competition in the presence of another molecule for a given amount of time. However, extraction and quantification is unique for each experiment depending on what molecule in the cascasde is being measured. The measurements are quantified either using radiometric, luminescence, fluorescence or absorbance [278]. The biggest advantages of functional assays over binding assays are that binding assays provide ligand affinity information, however this does not determine if the bound ligand results in activity. In contrast functional assays provide an answer to the effect on cellular function, so it has been argued that they provide a wider breath of information [276, 284]. 


\subsubsection{Enzyme Immunoassay}

Enzyme immunoassays (EIAs) and enzyme-linked immunosorbent assays (ELISAs) are assay systems that use antigens and antibodies labelled with an enzyme for the measurement of a specific substance in a biological sample. EIA can detect proteins, peptides and hormones in small quantities [285, 286]. Both EIA and ELISA are underpinned by the same theory and involve similar steps in practice, the difference in names is due to the names given in their first papers and patents. Engvall and Perlmann at Stockhol University, Sweden published the first ELISA paper in 1971 while Schuurs from NV Organon Laboratories, Netherlands filled patents for the technology as EIA in 1970s [287].

One of the most common methods to group the different EIAs is by the process of detection; either direct or indirect detection. The difference is the method in which the antigen (molecule of interest) is bound to the antibody of the plate and then detected [288]. Figure 3.3 shows an overview of each methodology.

In the direct EIA method, the antigen and the other constituents of the sample adsorb onto the base of a micro-well. Next an enzyme tagged antibody is incubated with the antigen, which binds to the antigen. Excess enzyme tagged antibody is removed by washing. This is followed by incubation with a substrate that produces a colour change due to the enzyme action. One of the key advantages of this technique is that it is very quick due to the use of one antibody. However, the requirement of an enzyme tagged primary antibody can make this very expensive. Also another problem is the reactivity of the antibody can be affected by conjugation [285, 289, 290].

In an indirect EIA the sample containing antigen adsorbs onto the base of the micro-plate, to which an antibody that is specific for the antigen of interest is added. This antibody is called the primary antibody. After washing to remove any unbound primary antibody, a second enzyme-conjugated antibody which binds to the primary antibody is introduced, a second wash removes any unbound enzyme conjugated antibody. A substrate for the enzyme is introduced to quantify the bound primary antibody through a colour change. The concentration of antigen is proportional to the intensity 
of colour generated. Indirect EIA has many advantages; there is a wide availability of enzyme conjugated antibodies that can be used in the second step. The loss of immuno-reactivity of the primary antibody is not a concern as it is not modified and different visualisation markers can be used for the same primary antibody. One of the biggest disadvantages of this method is cross-reactivity can occur with the secondary antibody and serum antigens, resulting in a nonspecific signal [285, 289, 290].

Further development of direct and indirect assays led to the development of sandwich and competitive EIAs [291]. The biggest difference is that the surface of the micro-well is coated with an antibody that binds to the antigen of interest.

In sandwich EIAs the sample containing the antigen of interest is introduced to the antibody coated wells. A second specific antibody is introduced that is also specific to the antigen, this "sandwiches" the antigen between the two antibodies. After washing an enzyme-conjugated secondary antibody is introduced, after a second washing step a substrate for the enzyme is introduced to quantify the antigen through a colour change. The concentration of antigen is proportional to the intensity of colour generated. Sandwich EIAs have the advantage of higher sensitivities as the antigen of interest is sandwiched between two antibodies [285, 289, 290]. Sandwich EIAs are considered to be the most robust therefore most commonly used [292].

Competitive EIAs use a similar methodology to sandwich based EIA, however when the sample containing the antigen of interest is introduced; a fixed concentration of enzyme linked antigen is also incubated at the same time. The enzyme linked antigen competes with the antigen from the sample for binding sites on the antibody. After washing only bound antigen is left behind and a substrate that produces a colour change upon transformation is introduced. If there is a larger concentration of antigen then a lower proportion of enzyme linked antigen is able to bind to the antibody, if the there is a low concentration of antigen then a higher proportion of enzyme linked antigen is able to bind to the antibodies. As the colour intensity is due to enzyme linked antigen, the colour intensity is an inverse proportion to the antigen of interest is within the sample. One of the greater advantages of these type of EIA is the ability to have higher sensitivities for smaller molecules [285, 289, 290]. This is the EIA technology used to quantify secondary messengers such as cAMP, inositol-trisphosphate $\left(\mathrm{IP}_{3}\right)$ and 
diacylglycerol (DAG) [293, 294, 295]. The competitive EIA was used within these studies to measure cAMP generated by $\mathrm{V}_{2} \mathrm{R}$, as this is the best EIA type to measure smaller molecules. 


\section{Direct}

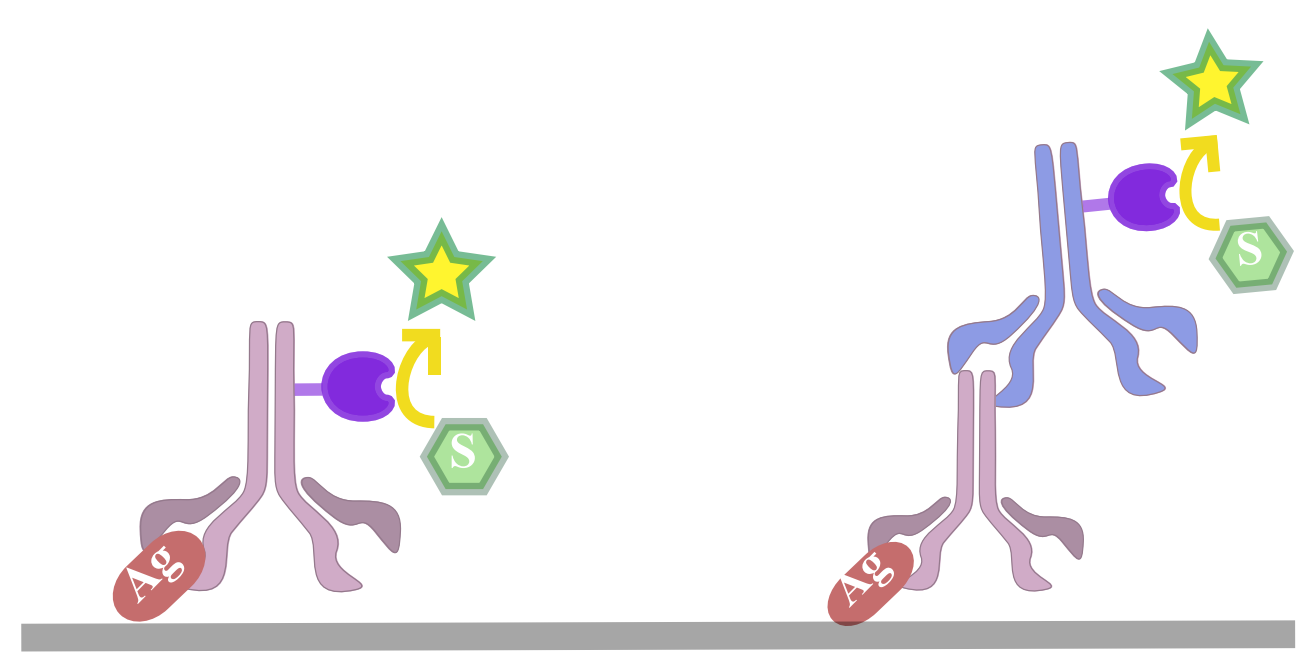

Sandwich

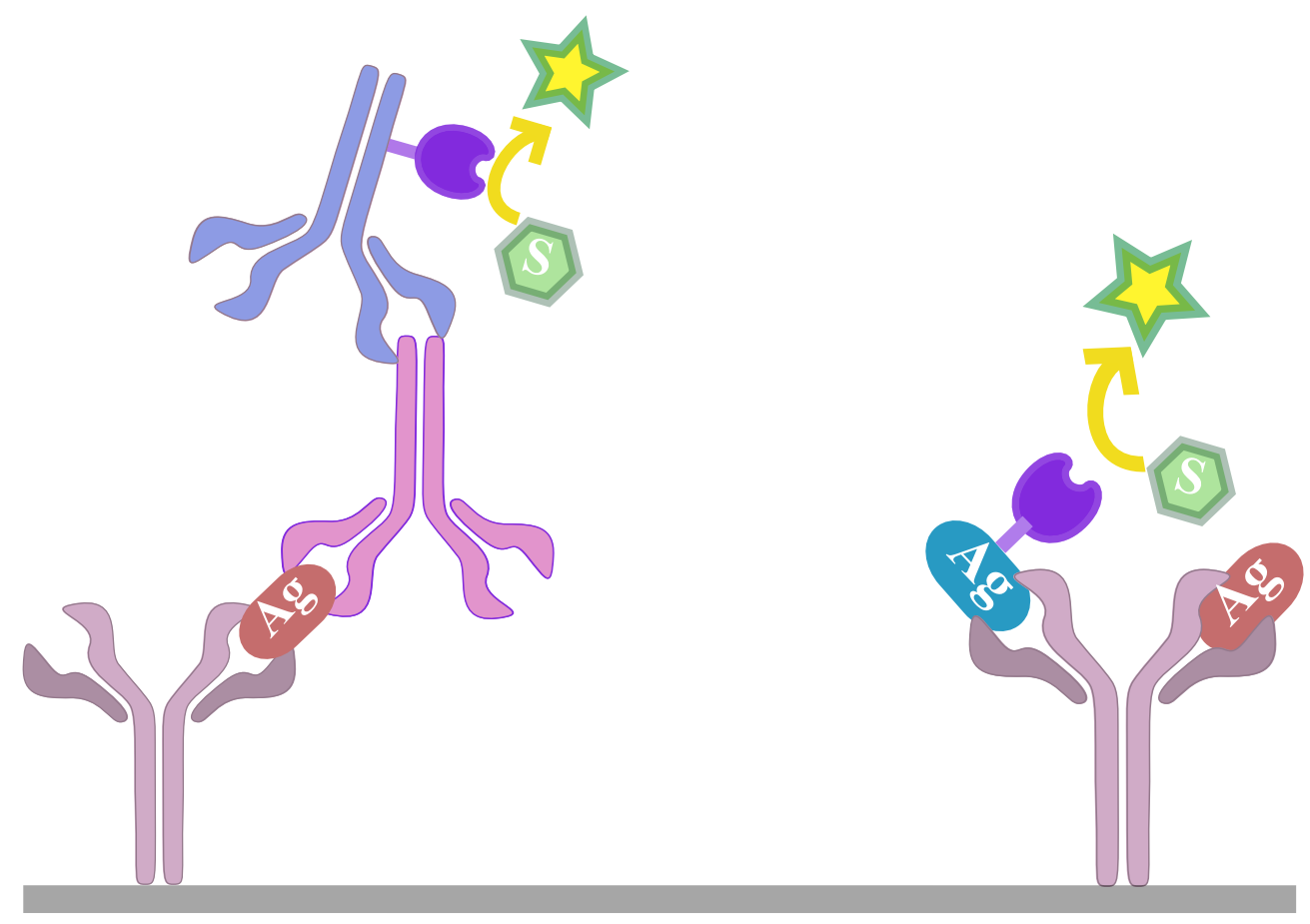

Indirect

Competitive 


\subsection{Materials \& Methods}

\subsubsection{Materials}

Table 3.2: Materials used for in vitro studies of METx.

\begin{tabular}{ll}
\hline Materials & Supplier \\
\hline Arg $^{8}$-Vasopressin & Sigma-Aldrich \\
cAMP EIA kit & GE Life Science \\
Dimethyl sulfoxide (DMSO) & Sigma-Aldrich \\
Eagle's Minimum & Sigma-Aldrich \\
Essential Medium (EMEM) & \\
Fetal Bovine Serum (FBS) & Sigma-Aldrich \\
Isobutylmethylxanthine (IBMX) & Sigma-Aldrich \\
L-Glutamine & Thermo Fisher Scientific \\
METx & Severn Biotech \\
MDCK Cell Line & European Collection of \\
& Authenticated Cell Cultures \\
Non-Essential Amino & Sigma-Aldrich \\
Acid (NEAA) & \\
Dulbecco's phosphate & Sigma-Aldrich \\
buffered saline (DPBS) & \\
Trypsin (0.25\%) & Thermo Fisher Scientific \\
\hline
\end{tabular}




\subsubsection{Methods}

The extent of activation or inhibition of $\mathrm{V}_{2} \mathrm{R}$ was determined by the accumulation of cAMP in Madin-Darby Canine Kidney (MDCK) cells. The accumulation was determined as described previously with a few modifications [296, 297, 298]. cAMP was measured using a competitive cAMP EIA kit, cAMP EIA Biotrack (GE Life Sciences). Data is expressed as fmol/well.

\subsubsection{Cell Culture}

MDCK cells were obtained from European Collection of Cell Cultures (ECACC) and cultured in Eagle's Essential Minimum Medium (EMEM) supplemented with fetal bovine serum (FBS 10\% v/v), L-Glutamine (1\% v/v) and non-essential amino acids (NEAA $1 \% \mathrm{v} / \mathrm{v})$. Cells were grown at $37^{\circ} \mathrm{C}$ in $\mathrm{T}-75 \mathrm{~cm}^{2}$ culture flasks under a humidified $95 \%$ air $15 \% \mathrm{CO}_{2}$ atmosphere. The cell culture medium was changed every 2 days. Cells were subcultured every 7 days or upon reaching confluence using trypsin /EDTA $(0.25 \%, 3 \mathrm{~mL})$ and reseeded at one third of their original density.

\subsubsection{Dose Response Studies}

MDCK cells (passage number 10-20) were detached as described above (section 3.2.2.1) using trypsin and seeded in the appropriate density depending on the plate size. Transwell plates (12 mm transwell with $0.4 \mu \mathrm{m}$ pore polycarbonate membrane insert, TC-treated) were seeded with 500,000 cells per transwell. Transwell plates were used as $75 \%$ of the $\mathrm{V}_{2} \mathrm{R}$ receptor are expressed in the basolateral membrane [267]. In the 24 well plates were seeded with 50,000 cells per well and 96 well plates were seeded with 100,000 cells per well as recommended by the manual of cAMP EIA and the cell plate specifications [299, 300] For experiments performed in 96 well plates, cells were seeded 16 hour before the experiments. Experiments in transwells or 24 well plates, cells were seeded 72 hours before the experiments, with cell culture medium changed everyday. 
On the day of the experiment, the cells were washed Dulbecco's phosphate-buffered saline (DPBS) without calcium and magnesium and incubated for 1 hour in cell culture medium with isobutylmethylxanthine (IBMX, $0.5 \mathrm{mM}$ ). IBMX is a phosphodiesterase inhibitor, this prevents the breakdown of cAMP within the cells, allowing for total quantification. After this, cells were incubated for 30 minutes with AVP or METx in cell culture medium with IBMX $(0.5 \mathrm{mM})$. For experiments performed in transwells both the apical and basolateral chambers were treated with the given concentration of AVP or METx. This is to reduce the concentration gradient of AVP or METx from the apical to the basolateral regions and vice versa.

After 30 min of incubation with the respective concentration of AVP or METx, the cells were lysed using the cAMP EIA's lysis buffer for 10 minutes as per the cAMP EIA protocol. cAMP was quantified from lysate $(100 \mu \mathrm{L})$ using a competition based EIA as per the protocol of the cAMP EIA. The optical density was measured using at $450 \mathrm{~nm}$ using ELx808 microplate reader (Bio-tek instruments, Potton, UK). Each concentration of AVP or METx was repeated in triplicate. The $\mathrm{EC}_{50}$ were calculated using Origin Pro 2016.

\subsubsection{Competition Studies}

The same experimental set up as described above (section 3.2.2.2) was followed for the seeding of MDCK cells and incubation of IBMX $(0.5 \mathrm{mM})$ on the day of the experiment. For competition studies, the cells was incubated with a high concentration of AVP and varied concentration of METx. After 30 minutes of incubation with the respective concentration of AVP and METx, the cells were lysed using the cAMP EIA's lysis buffer for 10 minutes as per the cAMP EIA protocol. cAMP was quantified from lysate $(100 \mu \mathrm{L})$ using a competition based EIA as per the protocol of the cAMP EIA. The optical density was measured using at $450 \mathrm{~nm}$ using ELx808 microplate reader (Bio-tek instruments, Potton, UK). Each concentration of AVP or METx repeated in triplicate. Each concentration of AVP and METx repeated in triplicate.

For experiments performed in 96 well plates, the concentration of AVP was $10^{-4} \mathrm{M}$ and the METx concentration was varied from $10^{-6}$ to $10^{-13} \mathrm{M}$. For experiments per- 
formed in transwells the concentration of AVP was $10^{-7} \mathrm{M}$ and the METx concentration was varied from $10^{-7}$ to $10^{-13} \mathrm{M}$. For experiments performed in 24 well plates the concentration of AVP was $10^{-4} \mathrm{M}$ and the METx concentration was varied from $10^{-7}$ to $10^{-13} \mathrm{M}$.

\subsubsection{Synergistic Studies}

Synergistic studies were performed in 96 well plates. The same experimental set up as described above (section 3.2.2.2 was followed for the seeding of MDCK cells in 96 well plates and incubation of IBMX $(0.5 \mathrm{mM})$ to prevent cAMP breakdown on the day of the experiment. Cells were incubated with either a high $\left(10^{-4} \mathrm{M}\right)$, medium $\left(10^{-5} \mathrm{M}\right)$ or low $\left(10^{-9} \mathrm{M}\right)$ concentration of AVP for 15 minutes, after which varied concentrations of METx, from $10^{-6}$ to $10^{-13} \mathrm{M}$, were added to the cells for a further 15 minutes. After the combined 30 minutes incubation the cell cells were lysed using the cAMP EIA's lysis buffer for 10 minutes as per the cAMP EIA protocol. cAMP was quantified from lysate $(100 \mu \mathrm{L})$ using a competition based EIA as per the protocol of the cAMP EIA. Each concentration of AVP or METx repeated in triplicate. The optical density was measured using at $450 \mathrm{~nm}$ using ELx808 microplate reader (Bio-tek instruments, Potton, UK). Each concentration of AVP or METx repeated in triplicate. 


\subsection{Results}

\subsubsection{AVP and METx Dose Response Curves}

\subsubsection{96 Well Plate}

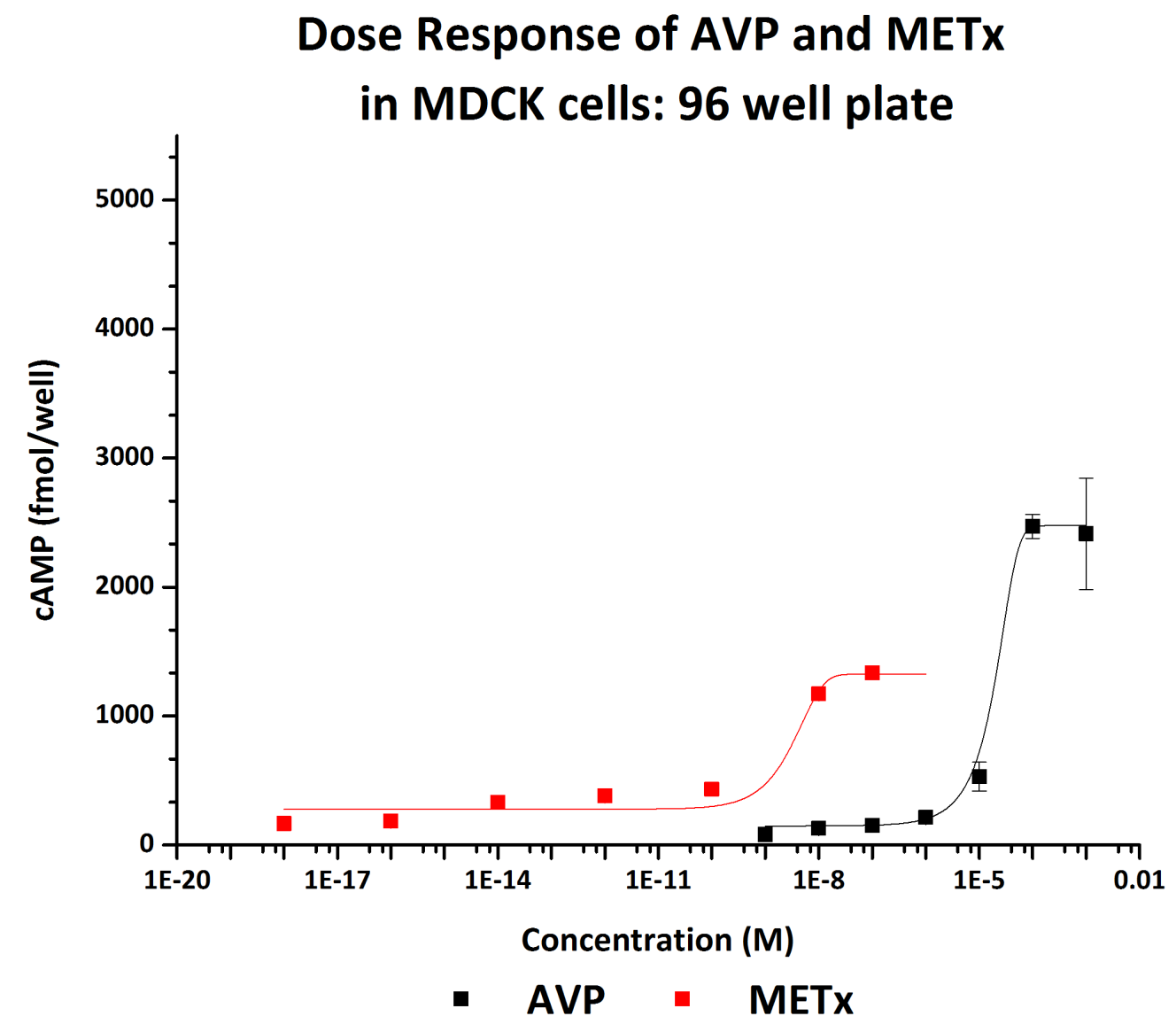

Figure 3.4: Dose response curve of AVP and METx in MDCK cells in 96 well plates.

The dose response of AVP and METx was performed in a 96 well plate with MDCK cells and the results are shown in figure 3.4. MDCK cells were incubated for 30 min with various concentration of AVP $\left(10^{-3}\right.$ to $\left.10^{-9} \mathrm{M}\right)$ or with various concentrations of METx $\left(10^{-6}\right.$ to $\left.10^{-18} \mathrm{M}\right)$, after which the cAMP was quantified using an EIA kit.

AVP produced a dose dependent increase in cAMP. The maximal response for AVP 
was seen at $10^{-4} \mathrm{M}$. The AVP does response curve's $\mathrm{EC}_{50}$ was $2.3 \times 10^{-5} \mathrm{M}$. METx also produced a dose dependent increase in cAMP, its maximal response was seen at a concentration of $10^{-8} \mathrm{M}$. The cAMP produced at $10^{-8} \mathrm{M}$ by METx was $55 \%$ compared to maximal amount of cAMP produced by AVP at $10^{-4} \mathrm{M}$, its top concentration. The $\mathrm{EC}_{50}$ for METx was calculated to be $1.4 \times 10^{-9} \mathrm{M}$. In comparison to AVP, the endogenous full agonist of the $\mathrm{V}_{2} \mathrm{R}$, METx produced a reduced maximal response in cAMP. This suggests that METx is a partial agonist of the $\mathrm{V}_{2} \mathrm{R}$ in MDCK cells. The $\mathrm{EC}_{50}$ of METx was calculated to be $5 \log$ units lower than that of AVP. The results suggest that METx may be a more potent partial agonist than AVP of the $V_{2} R$. 


\subsubsection{Transwell}

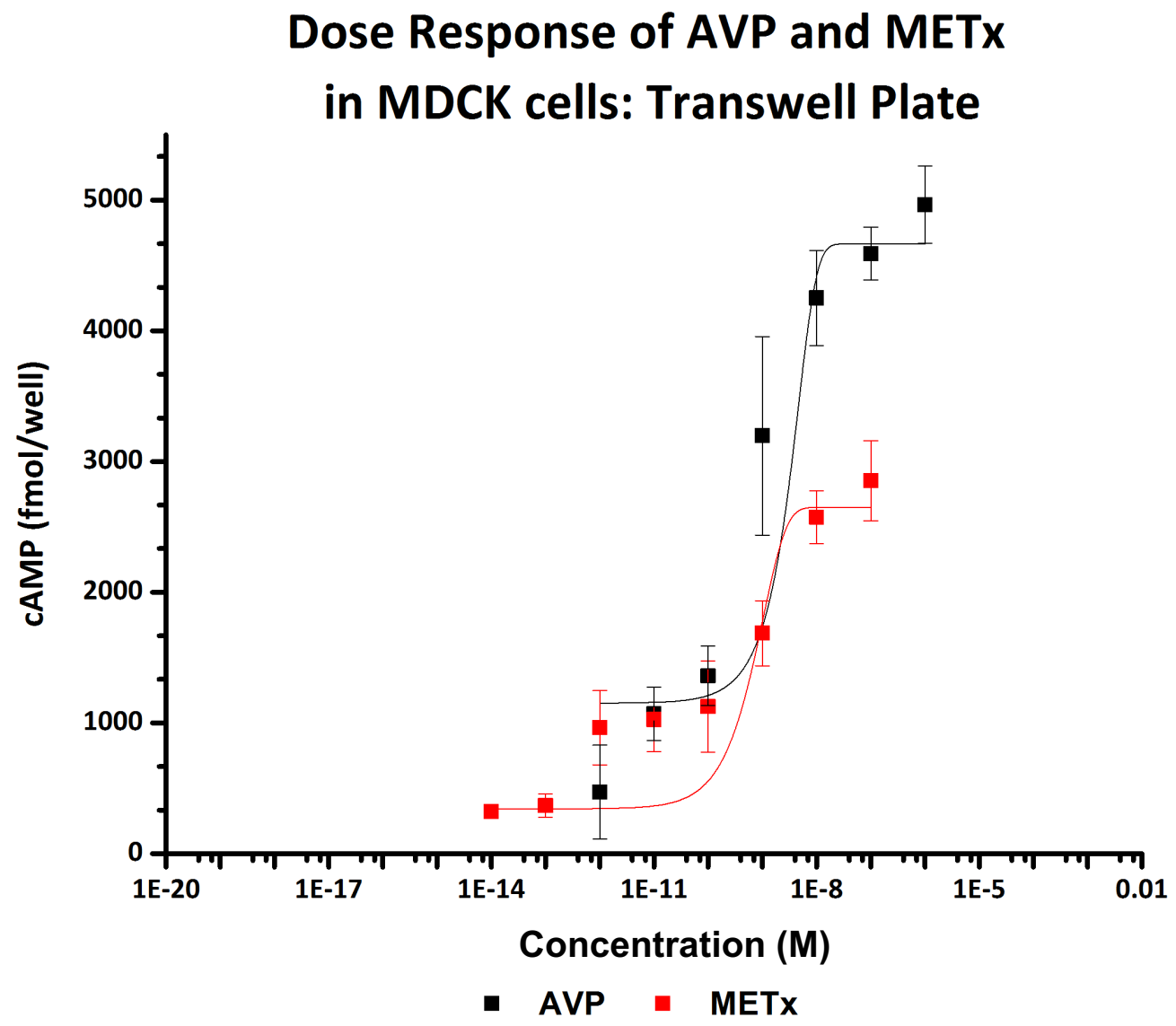

Figure 3.5: Dose response curve of AVP and METx in MDCK cells in 12 well transwell plates.

MDCK cells were seeded in transwell plates as $75 \%$ of the $\mathrm{V}_{2} \mathrm{R}$ receptor are expressed in the basolateral membrane [267], this allowed maximal access to the $\mathrm{V}_{2} \mathrm{R}$. MDCK cells were incubated with AVP and METx for 30 minutes in both apical and basolateral side for 30 min after which cAMP was quantified using a cAMP EIA kit. MDCK cells were incubated with AVP $\left(10^{-6}\right.$ to $\left.10^{-12} \mathrm{M}\right)$ or METx $\left(10^{-7}\right.$ to $\left.10^{-14} \mathrm{M}\right)$ for $30 \mathrm{~min}$.

Figure 3.5 shows the dose response curve of AVP and METx in the transwell. AVP produced a dose dependent increase in cAMP. The maximal response for AVP was seen at $10^{-7} \mathrm{M}$. The AVP does response curve's $\mathrm{EC}_{50}$ was $9.3 \times 10^{-10} \mathrm{M}$. METx also produced a dose dependent increase in cAMP, however its maximal response 
was seen at $10^{-8} \mathrm{M}$. The maximum amount of cAMP produced by METx was $57 \%$ compared to that of $\mathrm{AVP}^{\prime}$ s maximal cAMP response. The $\mathrm{EC}_{50}$ for METx was calculated to be $2.7 \times 10^{-9} \mathrm{M}$. In comparison to AVP, the endogenous full agonist of the $\mathrm{V}_{2} \mathrm{R}$, METx produced a reduce maximal response in cAMP. The results suggest that AVP and METx have the same level of potency for the $V_{2} R$ of the MDCK cells when seeded in transwell plates. METx showed partial agonist activity compared to AVP, the known full agonist of $V_{2} R$.

When the amount of cAMP produced by MDCK cells in transwells is compared to that of cells with the 96 well plates, those cells in the transwells produced a higher level of cAMP. The amount of cAMP generated in those cells seeded in the transwell plates are double that of the cells in the 96 well plates. It can also be seen that the $\mathrm{E}_{\max }$ was reached at a similar concentration for METx, however the $\mathrm{E}_{\max }$ concentration for AVP was far lower. 


\subsubsection{24 Well Plate}

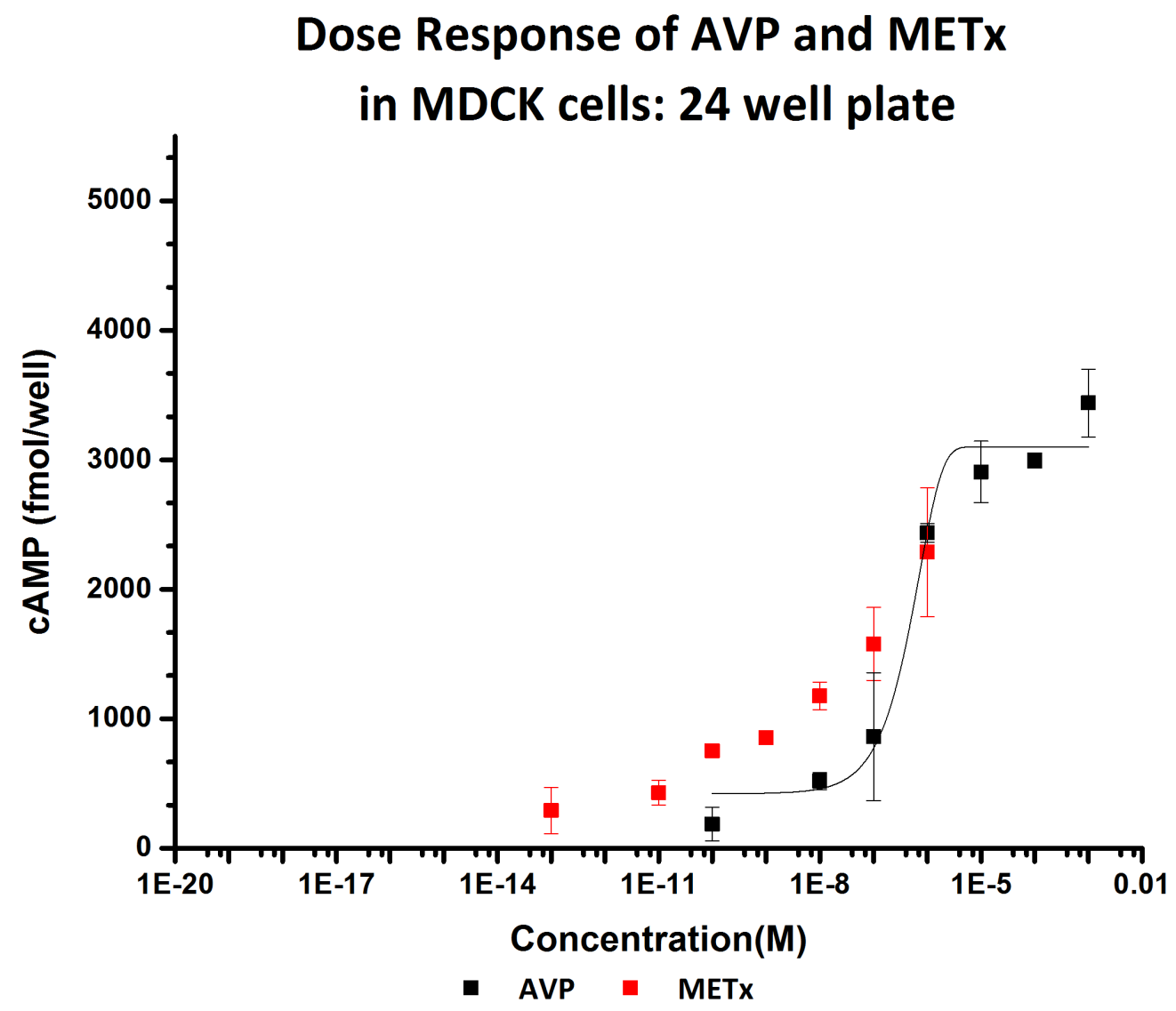

Figure 3.6: Dose response curve of AVP and METx in MDCK cells in 24 well plates.

MDCK cells were seeded in 24 well plates and grown in the same manner as the MDCK cells within the transwell dose response experiments. The 24 well plate was chosen as it had a similar growth area to that of the transwell plates, but without the polarised environment. This meant that the effect of the polarised cells could be further understood. Similar experiments to that of the transwell dose response curve were performed. MDCK cells were incubated with $\operatorname{AVP}\left(10^{-3}\right.$ to $\left.10^{-10} \mathrm{M}\right)$ or METx $\left(10^{-6}\right.$ to $10^{-13} \mathrm{M}$ ) for $30 \mathrm{~min}$.

Figure 3.6 shows the dose response curve of AVP and METx in the 24 well plate. AVP produced a dose dependent increase in cAMP. The maximal response for AVP was seen at $10^{-5} \mathrm{M}$. The AVP does response curve's $\mathrm{EC}_{50}$ was $1.7 \times 10^{-7} \mathrm{M}$. METx also produced a dose dependent increase in cAMP, however it did not plateau for a maximal 
response, which meant an $\mathrm{EC}_{50}$ could not be calculated. In comparison to AVP, the endogenous full agonist of the $V_{2} R$, METx did not reach a maximal response for the production of cAMP within the concentrations tested.

Table 3.3: $\mathrm{EC}_{50}$ of AVP and METx in different well plate.

\begin{tabular}{ccc}
\hline Well Plate & Molecule & $\mathrm{EC}_{50}(\mathrm{M})$ \\
\hline 96 well plate & AVP & $2.3 \times 10^{-5}$ \\
& METx & $1.4 \times 10^{-9}$ \\
Transwell & AVP & $9.3 \times 10^{-10}$ \\
& METx & $2.7 \times 10^{-9}$ \\
\multirow{2}{*}{ 24 well plate } & AVP & $1.7 \times 10^{-7}$ \\
& METx & ND \\
\hline
\end{tabular}

Comparison of the different $\mathrm{EC}_{50}$ values are shown in table 3.3 . It can be seen that as the the number of wells decrease from 96 to 24 well plates the $\mathrm{EC}_{50}$ values of AVP also decrease. The difference between the 96 and 24 well plate is an increased area, where as in the transwell plates there is an increased access to the $V_{2} R$. 


\subsubsection{AVP and METx Competition Experiments}

\subsubsection{96 Well Plate}

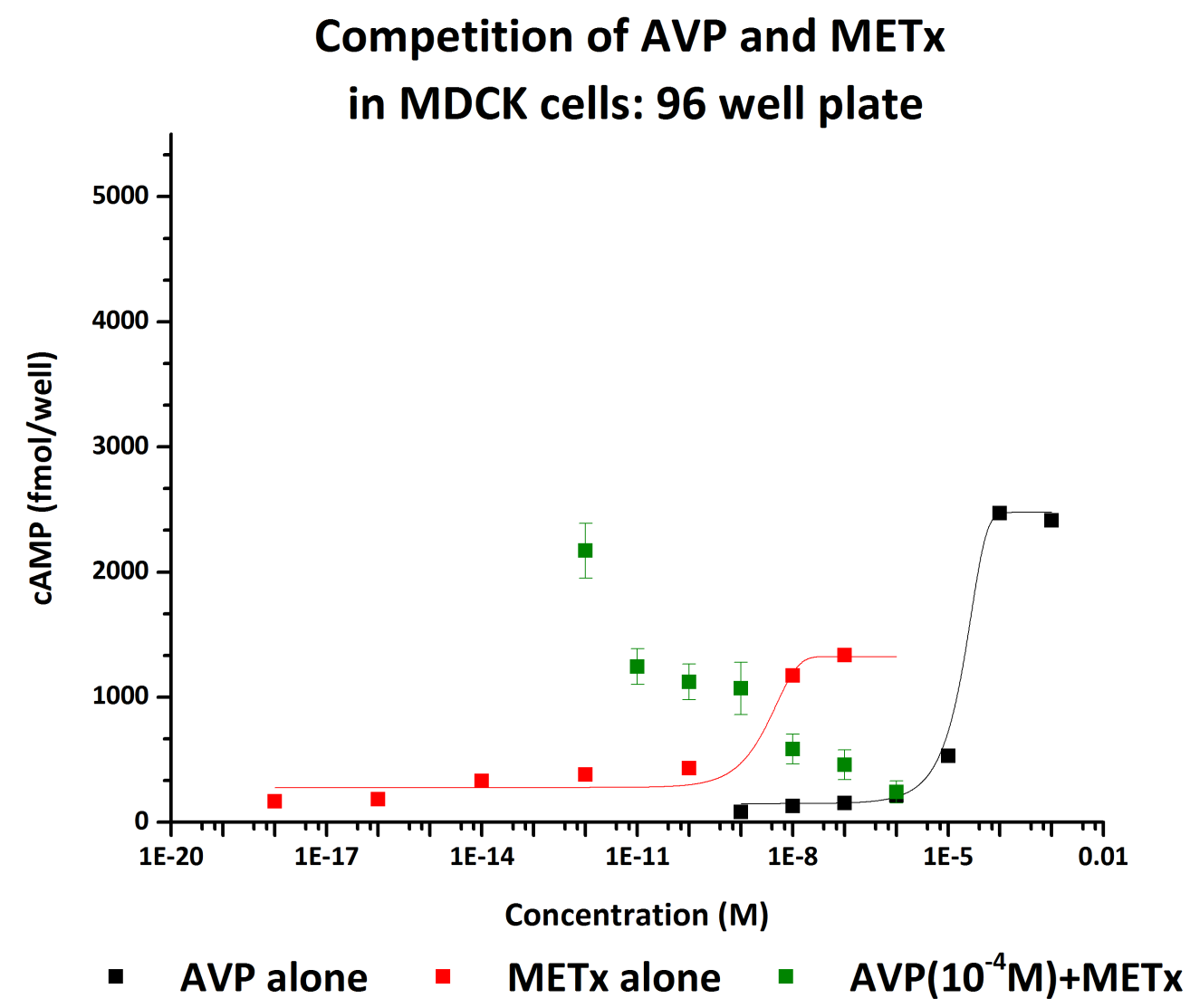

Figure 3.7: Competition experiment of AVP and METx in 96 well plates.

To better understand the activity of METx, experiments were performed to asses the competition of AVP and METx for the $\mathrm{V}_{2} \mathrm{R}$. This would help to determine if METx binds to the active site (orthosteric) or another site (allosteric) on the $\mathrm{V}_{2} \mathrm{R}$, as well as which has a higher affinity for $\mathrm{V}_{2} \mathrm{R}$. Cells were seeded in 96 well plates and treated with a high concentration of AVP $\left(10^{-4} \mathrm{M}\right.$ ) with various concentrations of METx (from $10^{-6} \mathrm{M}$ to $10^{-13} \mathrm{M}$ ) for $30 \mathrm{~min}$, after which the cAMP generated was quantified using a cAMP EIA kit.

The results can be seen in figure 3.7. As the concentration of METx in the presence of high concentrations of AVP increased the production of cAMP decreased. At 
low concentrations of METx and high concentration of AVP; the level of cAMP generated was very similar to that of high concentrations of AVP (figure 3.4). However, as the concentration of METx increased the production of cAMP decreases, reaching a level close to the basal level of cAMP production. The results from this experiment show that as the concentration of METx increases, it prevents the activation of the $\mathrm{V}_{2}$ suggesting METx in the presence of AVP is an antagonist of $V_{2} R$.

\subsubsection{Transwell}

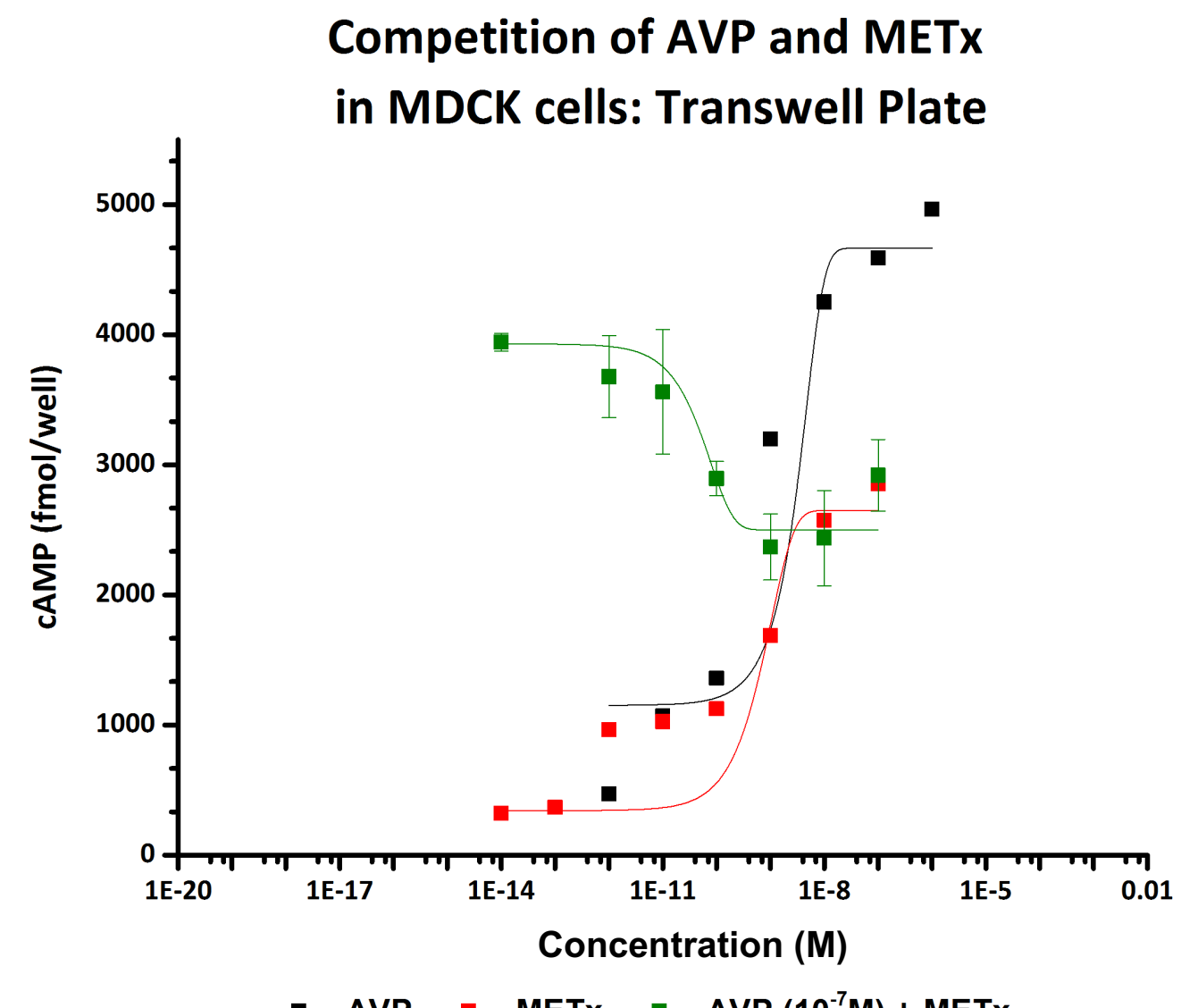

Figure 3.8: Competition experiment of AVP and METx in transwell. 
MDCK cells were seeded in transwells, allowing maximum access to the $V_{2} R$. Similar experiment to that seen in section 3.3.2.1 were performed with a few changes. The METx concentrations were varied from $10^{-7}$ to $10^{-13} \mathrm{M}$ and the high concentration of AVP incubated with each concentration of METx was $10^{-7} \mathrm{M}$, as this was the concentration close to the $\mathrm{E}_{\max }$ of AVP in the transwell plate (figure 3.5).

Those cells that were incubated with lower concentrations of METx and high concentrations of AVP; the cAMP response was similar to that of high concentrations of AVP alone like those of figure 3.5. As the concentration of METx increased, the cAMP production within the cells started to decrease stabilising to a similar level of that of the $\mathrm{E}_{\max }$ of METx. The decrease in cAMP production started to take place at similar concentration to the $\mathrm{EC}_{50}$ of METx. This can be seen in figure 3.8. This suggests that METx is a partial agonist that binds to the same active site of AVP of the $V_{2} R$ of MDCK cell lines. The results also suggest that METx has a higher affinity for $\mathrm{V}_{2} \mathrm{R}$ than AVP as cAMP production shifts to a similar level to that produced by METx.

When these results are compared to the 96 well plate experiment (figure 3.7); in the transwell plate the mix of high concentrations of AVP and METx still result in the production of cAMP whereas in the 96 well plate there is little production of cAMP. Within the transwell plate there is larger proportion of $\mathrm{V}_{2} \mathrm{R}$ that are more accessible compared to that of 96 well plate. This suggests that if enough $V_{2} R$ are available for both AVP and METx to bind to then partial activity is still possible. This shows the importance of a large enough number of $\mathrm{V}_{2} \mathrm{R}$ in order for METx and AVP to have activity in the presence of each other. 


\subsubsection{24 Well Plate}

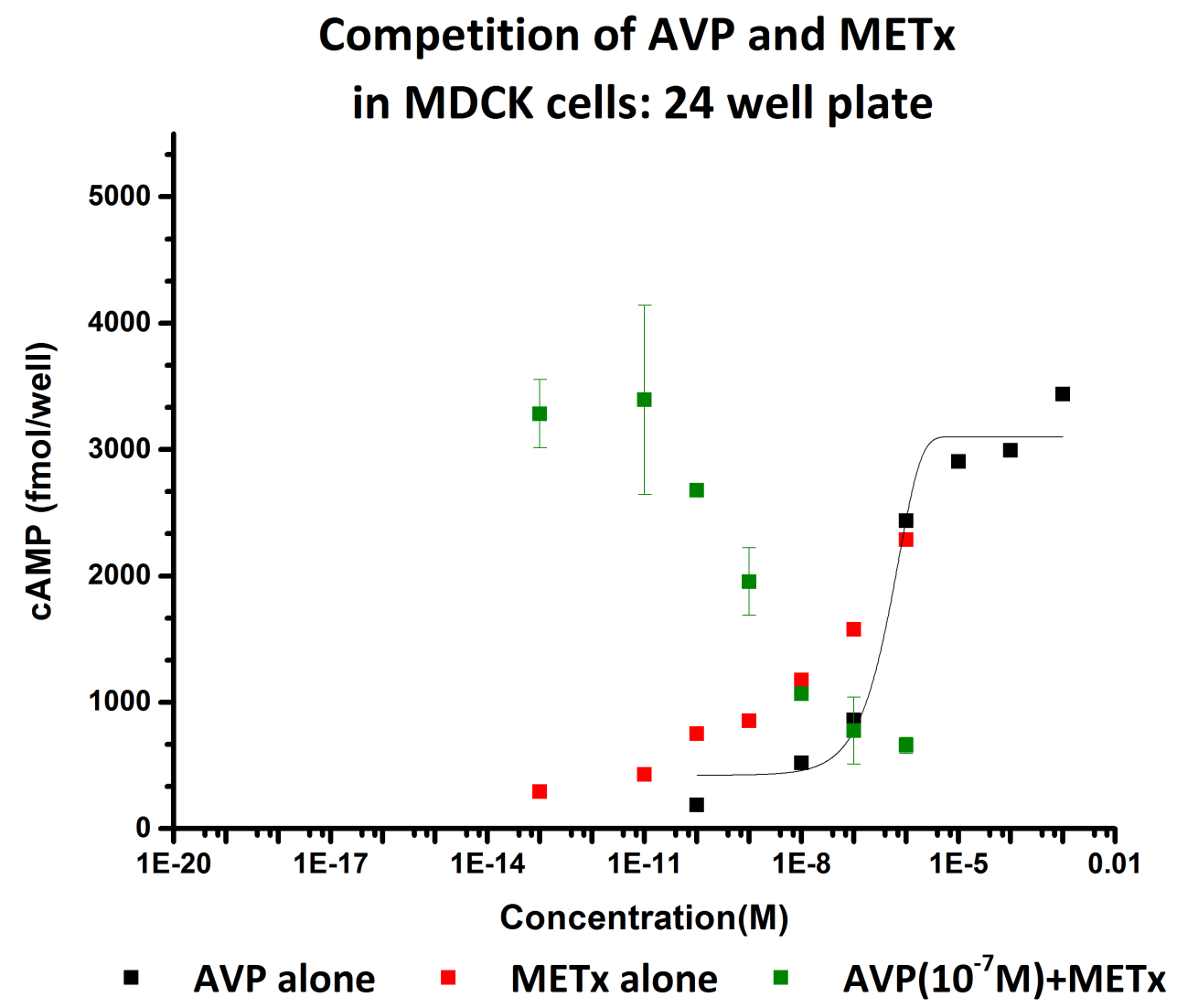

Figure 3.9: Competition experiment of AVP and METx in 24 well plate.

A similar experiment to that of 96 well plate was undertaken to understand the effect of METx in the presence of AVP in MDCK cells. This experiment also helped to understand the importance of the polarisation of the cells and the impact of a receptor reserve. Similar experiments were performed to that of section 3.3.2.1 with a few changes. The METx concentrations were varied from $10^{-6}$ to $10^{-13} \mathrm{M}$ and the high concentration of AVP incubated with each concentration of METx was $10^{-4} \mathrm{M}$. The different concentration of AVP was chosen as this was the concentration of AVP that produced the $\mathrm{E}_{\max }$ with the 24 well plate (figure 3.6.

The results can be seen in figure 3.9. As the concentration of METx increased the production of cAMP decreased. In those cells that were incubated with low concentrations of METx and high concentration of AVP; the level of cAMP generated was 
very similar to that seen in the 24 well dose response curves (figure 3.6. As the concentration of METx increased the level of cAMP decreased, reaching an amount close to the basal level of cAMP production. The results from this experiment suggest that METx is an antagonist of $V_{2} R$, as the highest concentrations of METx prevents cAMP production and inhibits the activity of AVP.

\subsubsection{Synergistic Studies}

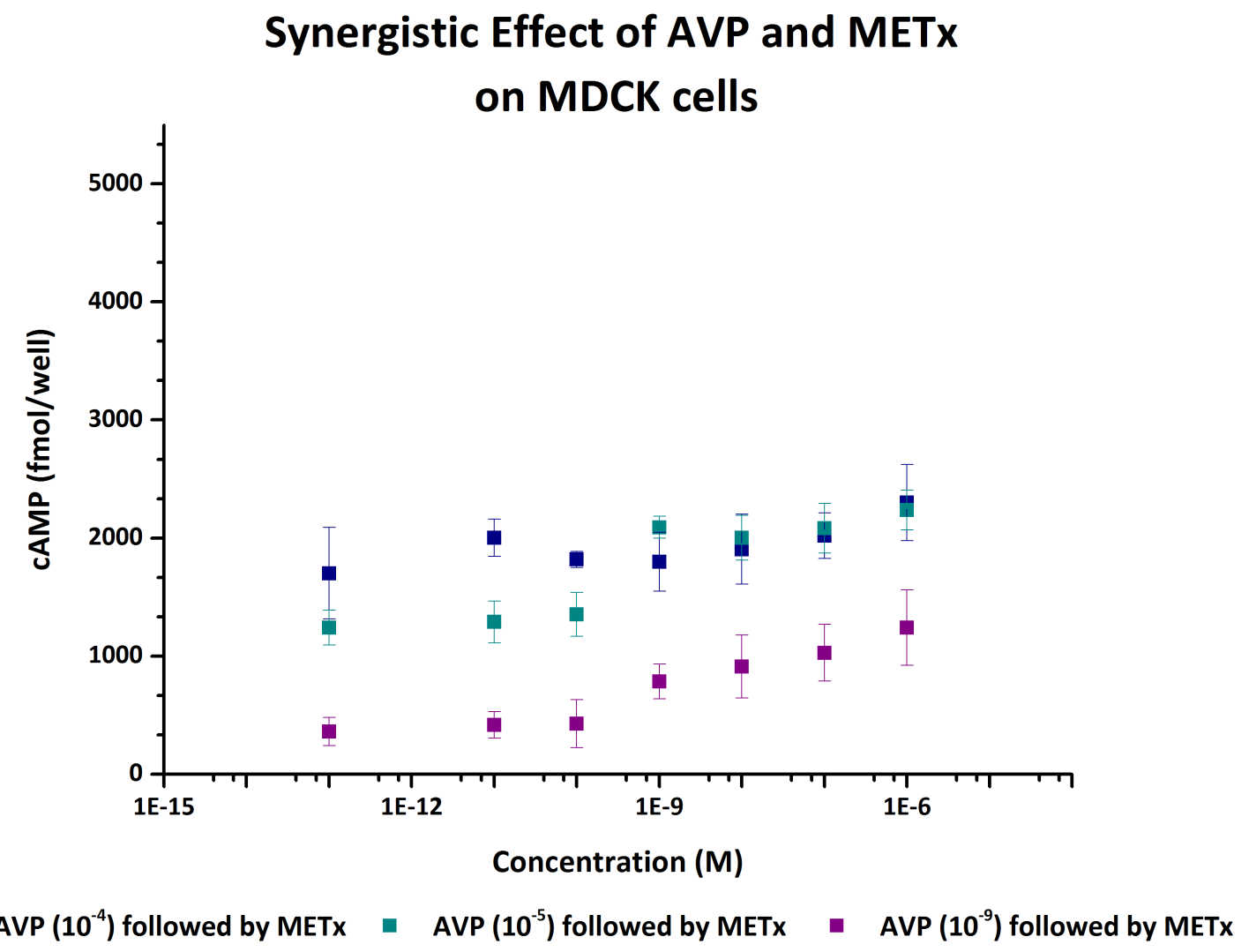

Figure 3.10: Synergistic effects of AVP and METx in 96 well plate.

Experiments were conducted to observe the effect of METx in MDCK cells that had been pre-incubated AVP in a 96 well plate. This was done to mimic the environment experienced within the body where there is constant presence of AVP. The body has a basal AVP level of 2-4 $\mathrm{pg} \mathrm{ml}^{-1},\left(2-4 \times 10^{-12} \mathrm{M}, 2-4 \mathrm{pM}\right)$ [301, 302] that can rise due to a number of stimuli of which the most potent is increased plasma osmolality [302]. MDCK cells were exposed to one of three concentrations (low, medium and 
high) of AVP for 15 minutes after which various concentrations of METx were added and incubated for further 15 minutes. The total exposure time was $30 \mathrm{~min}$ after which cAMP was quantified using an EIA kit. Three concentrations of AVP were used; below the $\mathrm{EC}_{50}\left(10^{-9} \mathrm{M}\right.$, low $)$, at the $\mathrm{EC}_{50}\left(10^{-5} \mathrm{M}\right.$, medium $)$ and a concentration above the $\mathrm{EC}_{50}\left(10^{-4} \mathrm{M}\right.$, high). The METx concentration range was from $10^{-6}$ to $10^{-13} \mathrm{M}$.

These results can be seen in figure 3.10. Where METx was added to those cells that had been pre-incubated with AVP concentrations below the $\mathrm{EC}_{50}$ and at the $\mathrm{EC}_{50}$; addition of METx caused an increase in the production of cAMP. The cAMP production increased at concentrations at and above the $\mathrm{EC}_{50}$ of METx $\left(10^{-9} \mathrm{M}\right)$. However, the cells that had been pre-incubated with the highest concentration of AVP $\left(10^{-4} \mathrm{M}\right)$; METx was not able to cause any increase in cAMP production. This showed that METx was still able to cause an increase the agonist effect in the presence of sub maximal AVP. The results also show that METx is not able to displace AVP from its receptor site. This can be seen from higher concentrations of METx that do not cause a decrease in the level of cAMP produced. If METx was able to displace AVP then this would result in a lower of cAMP at the higher concentrations as seen in figure 3.4 , however this does not happen. 


\subsection{Discussion}

The use of in vitro testing has become a key stage in the screening of ligands for GCPRs. The pharmaceutical industry have used in vitro testing to support the early identification of new drug candidates [303]. In vitro testing has also helped to provide an alternative method to some animal tests, thereby supporting the 3Rs (replacement, reduction and refinement) as part of UK Animals (Scientific Procedures) Act (ASPA) 1986 [304]. To further support the use and application of in vitro studies; the European Medicine Agency (EMA) has published a paper on the use of in vitro studies to reduce animal studies. The paper also provides information on the conditions required to ensure these methods and results can match with the requirements for regulatory approval [305].

On cells expressing $V_{2} R$ activation by AVP leads to the generation of cAMP, which further downstream leads to production of concentrated urine. The in vitro experiments discussed within this chapter were undertaken to understand the activity of METx on the $\mathrm{V}_{2} \mathrm{R}$ by measuring cAMP production within MDCK cells. There are two known cells lines that express the $\mathrm{V}_{2} \mathrm{R}$; MDCK cell line which is derived from the kidney of a dog and LLC-PK1 which is derived from the kidney of a pig [26]. Quantifying cAMP production using a competitive EIA determined the activation of $\mathrm{V}_{2} \mathrm{R}$. Incubating cells with IBMX before the experiment ensured that cAMP did not break down by inhibiting denosine 3,5-cyclic monophosphate phosphodiesterase [306]. Using functional assays instead of binding affinity assays helps to provide activity based information. For example, if METx showed affinity in a binding assay this would not necessarily mean that it has any pharmacological activity. A second experiment would have to be performed to understand if this binding would result in any form of activity.

In these experiments METx has shown partial agonist activity of the $V_{2} R$. In 96, 24 well and transwell dose response experiments, METx showed partial agonist activity, not reaching the same maximal response as AVP, the natural full agonist of $V_{2} R$. METx showed 60\% efficacy compared to AVP in the 96 well, 24 well and transwell format. There were no previous reports of the $\mathrm{EC}_{50}$ of $\mathrm{AVP}$ in MDCK cell, hence 
ours is the first such report. The values reported are for MDCK subclones or MDCK cells that have been transfected with genes to study the $V_{2} R$ or AQP-2 [307, 267, 308]. The literature values for $\mathrm{EC}_{50}$ of AVP in cells transfected with $\mathrm{V}_{2} \mathrm{R}$ line are between 0.6 to $18 \mathrm{nM}$ [296, 309, 310, 311], with similar values reported for primary culture of collecting ducts cells, $0.8-6.8 \mathrm{~nm}$ [312].

In all the dose response experiments (96, 24 well and transwell) METx showed partial agonist activity. The transwell experiment was used to assess the true potency and efficacy of METx as this is the physiologically relevant model, as seen by the similarity in $\mathrm{AVP}^{\prime} \mathrm{EC}_{50}$ value. The $\mathrm{EC}_{50}$ of $\operatorname{METx}\left(2.7 \times 10^{-9} \mathrm{M}, 2 \mathrm{nM}\right)$ is similar to that of AVP $\left(9.3 \times 10^{-10} \mathrm{M}, 0.9 \mathrm{nM}\right)$ and so METx can be considered to have a similar potency as AVP but a lower level of efficacy, around $60 \%$. The different $\mathrm{EC}_{50}$ values of AVP in the different well plates are shown in table 3.3 . The transwell plate has an $\mathrm{EC}_{50}$ value closest to physiological level, the 96 and 24 well plate are around 10,000 and 100 fold different.

With the knowledge that METx was a partial agonist the next series of experiments helped to understand more about the activity of METx. The next set of experiments helped to understand whether METx's site of action was the same as that of AVP, orthosteric site, or if it was on another part of the receptor, know as the allosteric site. Increasing concentrations of METx were incubated with a high concentration of AVP. If METx was a partial agonist that worked at the orthosteric site; the production of cAMP would drop closer to a similar level seen by METx, as METx would compete for the same active site as AVP. This lower level of cAMP production would be predominantly due to receptor activation by METx. However, if METx was to act upon an allosteric both the activity of METx and AVP would combine to give a larger production of cAMP.

Similar competition experiments have been heavily investigated with aripiprazole, a dopamine receptor partial agonist. Apriprazole is one of a few partial agonist drugs on the market, it is used as an antipsychotic for the treatment of schizophrenia and bipolar disorders [313]. Experiments were performed where large concentrations of dopamine, the natural agonist, with increasing concentrations of aprirpazole. As the concentration of apriprazole increased the overall dopamine response was reduced. 
This reduction showed that both dopamine and aripiprazole competed for the same active site [314, 315].

The experiment performed within the transwells showed that METx provided a level of functional antagonism of the natural AVP response as seen in figure 3.8. As the concentration of METx increased the overall production of cAMP was reduced. The cAMP was reduced to the same level as the METx $E_{\max }$ as the dose response curve (figure 3.5), when both AVP and METx are at high concentrations. This provided a suggestion that METx binds the the same active site as AVP and has a higher affinity than AVP for the $\mathrm{V}_{2} \mathrm{R}$ active site. This can be described as functional antagonism as METx reduces the cAMP production, therefore it reduces the response of AVP but still has its own agonist activity.

The same experiment that was performed at in a 24 (figure 3.9) and 96 (figure 3.7) well format suggested that METx was an antagonist, not a partial agonist. This is thought to be due to a lack of receptor reserve in the system. The receptor reserve is a percentage of receptors that are unoccupied when the system is expressing its maximal response. In the 24 and 96 wells at the high concentration of METx and AVP neither is able to create a cAMP response, this is thought to be as neither METx or AVP is able to bind effectively to the receptor to create a response. Thereby neither is able to create the production of cAMP.

The 24 well cell plate was used to mimic a similar environment to that of the transwell plate experiment with a lack of cell polarisation. The transwells used have a growth area of $1.12 \mathrm{~cm}^{2}$ and the 24 cells have a growth area $1.9 \mathrm{~cm}^{2}$, which is the closest of any multi-well format. Cells were seeded at the density recommended [299] and grown for 72 hours to become confluent after which the same experiment was performed. It is believed that the receptors are less accesible in this system; so as the concentration of METx increases, both METx and AVP compete for the same receptor site. However, neither is able to have a large enough concentration bind to the $V_{2} R$ such that a response in cAMP can be seen. As METx is suggested to have a higher affinity than AVP, it is able to bind to $\mathrm{V}_{2} \mathrm{R}$ but not in a large enough amount such that a response can be seen. 


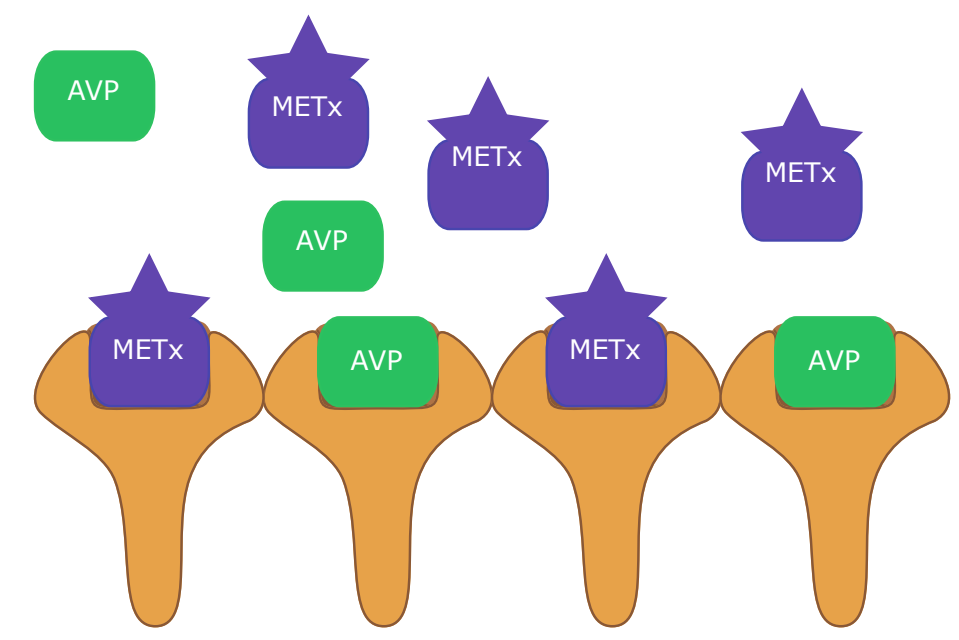

24 and 96 Well Plate

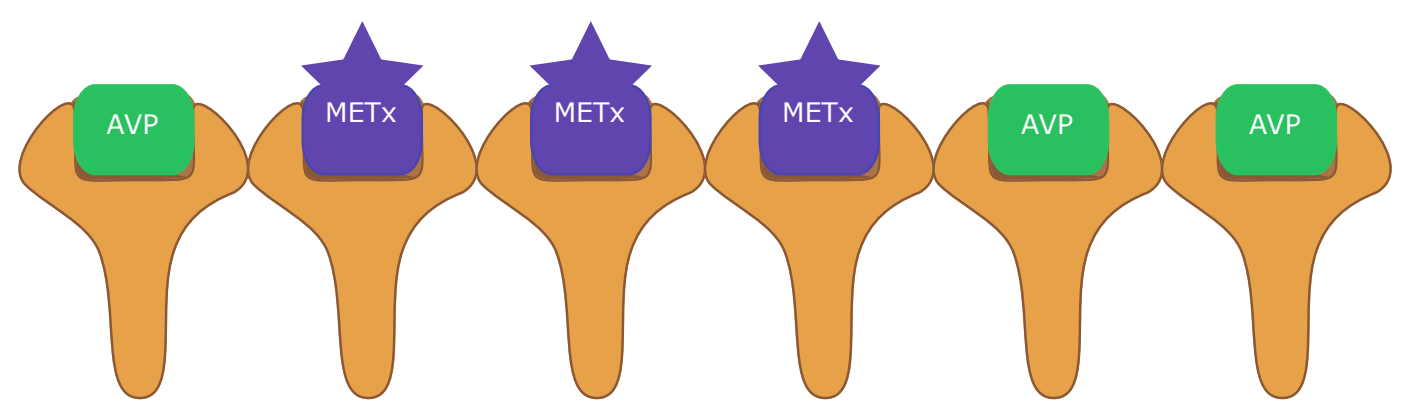

\section{Transwell Plate}

Figure 3.11: Receptor reserve of 24, 96 and transwell plates.

Figure 3.11 shows a diagram of the conditions within the 24, 96 and transwell plates. Within the 24 and 96 well plate there are not enough receptor binding sites for METx or AVP thereby neither is able to create a response. Where as in the transwell plate; there are enough accessible binding sites such that no METx or AVP is left unbound, but as METx is a partial agonist, only a partial number of receptors bound will become fully activated resulting in a partial response.

Within a 96 well plate the ability for METx to displace AVP was studied. MDCK cells were incubated with low (below $\left.\mathrm{EC}_{50}\right)$, medium $\left(\mathrm{EC}_{50}\right)$ and high (above $\mathrm{EC}_{50}$ ) concentrations of AVP. After which various concentrations of METx were incubated 
to challenge the effect of AVP. The results are shown in figure 3.10. METx was able to show an effect at in the two lower concentrations, the effect was seen at concentrations near and above the $\mathrm{EC}_{50}$ of METx. However in the highest concentration METx did not have any effect. This suggest that METx is not able to displace AVP, but it able to increase the effect if AVP has not reached a maximal effect. These results are different to those in the competition transwell experiment (figure 3.8) where METx is able to compete with AVP for the $\mathrm{V}_{2} \mathrm{R}$.

The experiments from this chapter suggest that METx is a partial agonist of the $\mathrm{V}_{2} \mathrm{R}$. METx is a hydrophobic derivate of OXT. Although most of the more recent work into the discovery of newer vasopressin agonists have focused on small molecules [23, 22], there is much evidence to suggest peptide based research should still be considered.

$\mathrm{V}_{2} \mathrm{R}$ agonist are the first line treatment in DI. DI is linked to either a reduced secretion of AVP or an increased resistance to AVP in the kidneys [35]. The main stay treatment for diabetes insipidus is desmopressin. Desmopressin is synthetic vasopressin analogue that is selective for the $\mathrm{V}_{2} \mathrm{R}$. Desmopressin, (1-desamino 8, d-Arg)vasopressin, was first discovered in the late 1960s and is a peptide based vasopressin agonist [316]. Desmopressin is still currently the most potent $\mathrm{V}_{2} \mathrm{R}$ agonist in therapeutic use. Desmopressin is a full agonist that is more potent than AVP on the $V_{2} R$ [317]. Another vasopressin based analogue discovered in the late 1950s from pigs is lypressin, (8-lysine)-vasopressin. Lypressin was found to have a lower potency than AVP and desmopressin, with lypressin having about $10 \%$ activity compared to AVP [318].

Side effects of hyponatremia and anti-diuresis have been reported from OXT infusions since the early 1960s [319, 320, 321]. Studies have shown OXT has affinity for the AVP receptors and can cause antidiuretic effects like AVP. OXT has been previously shown to increase water permeability at the collecting ducts of rats when given at physiological and at concentrations above physiological levels [322]. Another study by Han et al. measured cAMP production in isolated collecting ducts. OXT $\left(10^{-8} \mathrm{M}\right.$, $10 \mathrm{nM})$ generated around 50\% cAMP level compared to AVP (10 nM) [323]. This cAMP levels are similar to that seen by METx. As the structure of METx is based on 
OXT, the partial activity suggests that the structure activity of METx is not lost by the addition of lys-(palmitoyl) at position 10. These literature reports also help to explain the partial activity seen by METx.

To further understand the mechanism of OXT and its antidiuretic effect, OXT was infused into Brattleboro rats. Brattleboro rats are genetic knockout rats that cannot produce AVP. OXT infusion resulted in reduced urine production which was inhibited by $\mathrm{V}_{2} \mathrm{R}$ antagonists, but not by $\mathrm{OXT}$ antagonist. This suggested that $\mathrm{OXT}^{\prime}$ s antidiuretic effect was activity on the $\mathrm{V}_{2} \mathrm{R}$. Further experiments found that $\mathrm{OXT}^{\prime} \mathrm{s}$ antidiuretic mechanism of action was similar to that of AVP with increased proteins levels of AQP2 and phosphorylated AQP2 [324].

Considering this information and early promises with the use of OXT based molecules to discover newer antidiuretic drugs, very few studies were done. One such study by Berde et al. found that replacing isoleucine at position 2 with phenylalanine produced an analogues that had reduced OXT effects but increased antidiuretic effects compared to OXT itself [325]. The majority of the research for newer, more potent, more selective vasopressin agonists have focused on small molecules. The use of benzodiazepine structures has formed the foundation of the majority of the molecules. This is because benzodiazepine has been referred to as the "privileged structure" for AVP agonists [326].

Researchers at Pfizer have developed VNA-932, pyridobenzodiazepines based molecules for $\mathrm{V}_{2} \mathrm{R}$ agonist. This was developed from lixivaptan (VPA-985) an orally active $\mathrm{V}_{2} \mathrm{R}$ antagonist, being developed for hyponatremia and heart failure [327]. VNA-932 and its derivatives were discovered from structure activity relationships of lixivaptan [328, 329]. However as these compounds were not compared to either desmopressin or AVP directly comparisons cannot be made of their potency to that of METx.

Kissei Pharmaceutical, a Japanese pharmaceutical company, have developed two other benzodiazepine derivates that are full agonists of $\mathrm{V}_{2} \mathrm{R}$ with $\mathrm{EC}_{50}$ values similar to that of AVP [326]. This would exhibit a similar potency to METx, but with higher efficacy, if the $\mathrm{EC}_{50}$ and activation percentages are compared. Ferring Pharmaceut- 
icals have also researched this area, having developed an OXT antagonist (atosiban), approved for preventing premature labor [27]. Development of a benzodiazepine with a urea moiety led to the discovery of VA106483. The molecules developed were compared to AVP (100nM) in a luciferase production assay, with VA106483 showing the profile of a full agonist with a similar $\mathrm{EC}_{50}$ to that of AVP [330]. This would also show similar potency to METx, however with high efficacy.

In vitro experiments on MDCK cells have led to the discovery of METx's partial agonist properties. Desmopressin has become the main treatment for DI. Desmopressin is well tolerated by the majority of patients, however the risk of severe adverse effects, such as hyponatraemia and water intoxication require a strict fluid intake with little room for variability [60]. Although desmopressin is more potent than AVP [317], thus making it more potent than METx; METx has an advantage that as partial agonist, it causes sub maximal activation. This sub maximal activation may help to decrease the number of side effects caused by full agonist activity on the receptor [331]. 


\section{Chapter 4: Formulation Development}

\subsection{Introduction}

The oral route for any medication is the most convenient and the one with the most patient compliance. It allows for patient convenience and lowers manufacturing costs as the degree of sterility required is minimal [73]. In order to be able to deliver peptides orally, they first need to be protected from the harsh environments of the GI tract followed by a route that allows for uptake into the systemic circulation [332]. They also need to be formulated such that they remain stable in the plasma in order to reach the site of action. Most peptides including those released from within the body have very short half-lives of minutes [333], therefore these peptides need to be administered a number of times during the day or over a continuous period. Patients do not like administering injections many times each day [334].

The polymer of this project is based on a glycol chitosan (GC) derivative. Chitosan is one of the most commonly used natural polymers for the production of nanoparticles [335]. Chitosan is prepared from chitin by deacylation of $\mathrm{N}$-acetyl glucosamine. The removal to the acetyl can be performed chemically or with enzymes, using chemicals is preferred due to low cost and the ability to scale up production [336]. When the degree of deacylation drops below $60 \mathrm{~mol} \%$, chitin is classified as chitosan [337]. Compared to the vast number of polymers that have a monograph in the pharmacopeia, chitosan is very unique, in that it is the only polymer that is cationic. This cationic property is due to the amino group and is responsible for many of its useful drug delivery properties [338]. 
Chitosan has been extensively studied to develop drug delivery systems [338, 339. [340]. Chitosan has been shown to increase membrane permeability in vitro and in vivo due to a mucoadhesive effect [339] as well as increase circulation time in the plasma [341]. Chitosan has been used to make formulations for oral, subcutaneous, transdermal and ophthalmic drug delivery [174, 342, 343]. Hydrophobically modified chitosan derivates have been designed to increase the solubility of poorly soluble drugs [337].

GC is derived from chitosan by conjugation of an ethylene glycol branch to the hydroxyl group at position 6 of the sugar ring. This helps in to increase glycol chitosan's solubility at neutral $\mathrm{pH}$ [344]. Quaternary ammonium palmitoyl glycol chitosan (GCPQ) is a GC based polymer [345]. GCPQ has been shown to improve the oral delivery of peptides and hydrophobic drugs that have very little or no bioavailability [139, 183, 182].

\subsubsection{Polymer Characterisation Methods}

\subsubsection{Nuclear Magnetic Resonance (NMR)}

NMR is a spectroscopic technique that uses radio frequency to provide structural information about the molecule and the chemical environment of hydrogen or carbon atoms depending on the technique used [346].

The theory of NMR is based on nuclei with an odd atomic number, these nuclei have a nuclear spin. This nuclear spin is characterised by a nuclear spin quantum number, known as "I" [347]. The spin of nuclei of different elements is individual enough such that NMR experiments can be sensitive towards one particular isotope of a specific element. Using this basis researchers have used the behaviour of ${ }^{1} \mathrm{H}$ and ${ }^{13} \mathrm{C}$ to gain structural information of organic compounds [348]. Proton $\left({ }^{1} \mathrm{H}\right)$ NMR is the most commonly used form of NMR due to its sensitivity and the amount of structural information that is generated [349].

A nucleus with an odd atomic number is considered to be a charged particle in 
motion. ${ }^{1} \mathrm{H}$ and ${ }^{13} \mathrm{C}$ have nuclear spins of $\mathrm{I}=1 / 2$ and behave similarly to that of a tiny bar of magnet. When there is no magnetic field applied they are in a random orientation. When a magnetic field is applied they can either align with or against the magnetic field [348].

In presence of an external magnetic field two spins states can exist either $+1 / 2$ (aligned with the magnetic field) or $-1 / 2$ (aligned against the magnetic field). The energy difference between these two states is very small. The greater the strength of the magnetic field the greater the energy difference. This energy difference is given as frequency units $(\mathrm{MHz})$ [350].

NMR data is not expressed as frequency as no two magnets will have exactly the same magnetic field. This means the data generated between two different NMR machines will not be comparable unless it is normalised. To solve this problem all NMR signals are relative to a reference signal from a standard compound, tetramethylsilane (TMS). This generates a very sharp peak as it contains 12 hydrogens in the same chemical environment, the normalised data is called chemical shift, with ppm as its units [346].

Chemical shift is the position of signal along the $\mathrm{x}$ axis of an NMR spectrum. The chemical shift indicates the structural environment of the protons producing the signal, the intensity of each peak indicates the number of protons in the same chemical environment [351]. The atoms surrounding a carbon can either shield or deshield the protons from the magnetic field. Those protons that are shielded will resonate at a higher frequency and so be expressed downward in the NMR specta. This provides an idea of their chemical environment. The number of peaks, their position and their intensity, provides information on the chemical structure of a molecule [352].

\subsubsection{Gel Permeation Chromatography (GPC)}

GPC is a method that allows for the separation, purification and analysis of a mixture of substances based on their molecular size [353]. It allows for the molecular weight and the molecular weight distribution of a polymer sample to be determined [354]. During 
GPC a polymer solution is passed through a chromatography column that is packed with porous particles. The separation process takes place due to the penetration of smaller molecules into the pores of the column packing material [354]. The smaller molecules enter the pores within the column and the larger molecules are not able to penetrate the pores so are eluted from the column more quickly. Therefore the pore size of the beads within the column determines the separation [355].

The components that are eluted are detected by a number of methods; these results are displayed in a graph. One such approach is to record the elution volume and then convert this to elution time for ease of comparison. This data is then compared to a calibration curve for the elution time of polymers of which the molecular weight is known [355].

Static light scattering is another technique used to measure the molecular weight. It utilises a relationship between the intensity of light scattered by a molecule and its molecular size and weight. This ratio is based on the Rayleigh theory which states the molecular weight of a molecule is proportional to the Rayleigh ratio of scattered light [356].

$\mathrm{Dn} / \mathrm{dc}$ also called the specific refractive index increments, is a measurement of how the refractive index of a polymer solution changes with the concentration of solute (polymer). It is important to measure $\mathrm{dn} / \mathrm{dc}$ as it is a term required to calculate molar mass [357]. If the concentrations of solutes are known the following equations are used to calculate the Mw:

$$
\mathrm{K}_{\mathrm{c}} / \mathrm{R}_{\Theta}=1 / \mathrm{Mw}+1 / \mathrm{Mw}\left(16 \pi^{2} / 3 \lambda^{2}\right) \sin ^{2}(\Theta / 2)(<\mathrm{r}>\mathrm{z})^{2}+\mathrm{A}_{2} \mathrm{c}
$$

where $\mathrm{c}$ is polymer concentration; $\mathbf{R}_{\Theta}$ is the Rayleigh ratio at the angle $\Theta$; Mw is the weight averaged molecular weight; $\lambda$ is the wavelength of light, $\Theta$ is the scatter angle; rz the z-averaged mean radius of gyration and the A2 the second viral coefficient 
which quantifies the interaction between the polymer and the solvent [356, 358].

$$
\mathrm{K}_{\mathrm{c}}=2 \pi^{2} \mathrm{n}^{2}(\mathrm{dn} / \mathrm{dc})^{2} / \mathrm{N}_{0} \lambda^{4}
$$

Where $\mathrm{N}_{0}$ is the Avogardo's number; $\mathrm{n}$ is the refractive index of the solvent and $\mathrm{dn} / \mathrm{dc}$ is the refractive index increments of the solute the change in refractive index per change in polymer concentration [356, 358].

\subsubsection{Formulation Characterisation Methods}

An ideal ratio of polymer to peptide has to be determined in order to prepare the optimum formulation. Having a greater ratio of polymer does not always help, as larger ratios of polymer may lead to a reduction in encapsulation, protection and undesired particle sizes [188, 359]. The size of a particle produced will also influence its biodistribution within the body [215, 360].

\subsubsection{Dynamic Light Scattering (DLS)}

The size of the nanoparticles formed is very crucial. Particle size influences a particle's physiochemical properties and a particle's biodistribution in the body. After administration particle size helps determine particle destination, nanoparticles that are 150$300 \mathrm{~nm}$ in size are generally found in the liver and spleen [360] while particles that are $30-150 \mathrm{~nm}$ in size are found in the bone barrow, heart, kidney and stomach [215].

Size can be measured by a number of techniques, such as DLS or nanoparticle tracking analysis (NTA). In this work DLS was used. DLS utilises Brownian motion and then correlates it to the size of particles; hence it requires particles to be suspended within a liquid. The larger the particle the slower its Brownian motion. This is because the smaller particles are moved further and more rapidly by solvent molecules [361]. Within the DLS, a laser beam is passed through the suspension of particles. As the particles pass through the laser beam it becomes scattered, this causes variations of the laser beam intensity at the detector over time. The variations in the intensity of the 
laser beam at the detector are used to calculate the particle size [362].

The size of a particle is calculated using the translation diffusion coefficient using the Stokes-Einstein equation.

$$
\mathrm{d}(\mathrm{H})=\mathrm{kT} / 3 \pi \eta \mathrm{D}
$$

Where $\mathrm{d}(\mathrm{H})$ is hydrodynamic diameter; $\mathrm{D}$ is the translational diffusion coefficient; $\mathrm{k}$ is the Boltzmann's constant; $\mathrm{T}$ is absolute temperature and $\eta$ is viscosity [361].

The measurement by the DLS of the particle size is called the hydrodynamic diameter, which is defined as "the size of a hypothetical hard sphere that diffuses in the same fashion as that of the particle being measured". Although this is difficult as normally particles are not fully spherical, are in dynamic motion and free in solution. The diameter of the particle is calculated from the diffusional properties of the particle and this provides an indication of the solvated particle in the medium, this is called the hydrodynamic diameter. Hydrodynamic diameter is also sometimes referred to as Stokes diameter [363]. 


\subsection{Materials \& Methods}

\subsubsection{Materials}

Table 4.1: Materials used for formulation development of METx.

\begin{tabular}{|c|c|}
\hline Materials & Supplier \\
\hline Acetic Acid (Glacial) & Fisher \\
\hline Amberlite IRA 140 & Sigma-Aldrich \\
\hline Amberlite IRA 96 & Sigma-Aldrich \\
\hline Bovine Serum Albumin & Sigma-Aldrich \\
\hline Chloroform & Sigma-Aldrich \\
\hline Dextrose & Sigma-Aldrich \\
\hline Dialysis Membrane & Medicell \\
\hline Diethyl Ether & Sigma-Aldrich \\
\hline Dimethyl sulfoxide & Sigma-Aldrich \\
\hline Dimethylformamide & Ratburn \\
\hline Ethanol (Abs) & FIsher \\
\hline Glycerol & Fisher \\
\hline Glycol Chitosan & Wako \\
\hline Hydrochloric Acid (32\%) & Fisher Scientific \\
\hline METx & Severn Biotech \\
\hline Methyl Iodide & Sigma-Aldrich \\
\hline Milli-Q Water & Merck Millipore \\
\hline N-Methyl-2-pyrrolidone & Sigma-Aldrich \\
\hline Pepsin & Sigma-Aldrich \\
\hline Phosphate Buffer Saline & Sigma-Aldrich \\
\hline Palmitic acid N-hydroxysuccinimide & Toronto Research Chemical Inc \\
\hline Saline & Severn Biotech \\
\hline Sodium Acetate (anyhydrous) & Sigma-Aldrich \\
\hline Sodium Bicarbonate & Sigma-Aldrich \\
\hline Sodium carbonate & Sigma-Aldrich \\
\hline
\end{tabular}


Sodium chloride

Sodium Hydroxide

Sodium Iodide

Sodium Nitrate

Triethylamine
Sigma-Aldrich

Sigma-Aldrich

Sigma-Aldrich

Sigma-Aldrich

Sigma-Aldrich 


\subsubsection{Methods}

\subsubsection{Polymer Synthesis \& Characterisation}

Figure 4.1 shows an overview of the reactions performed to synthesis GCPQ from GC and figure 4.2 shows the reaction of palmitoylation of GC using palmitic acid N-hydroxysuccinimide.

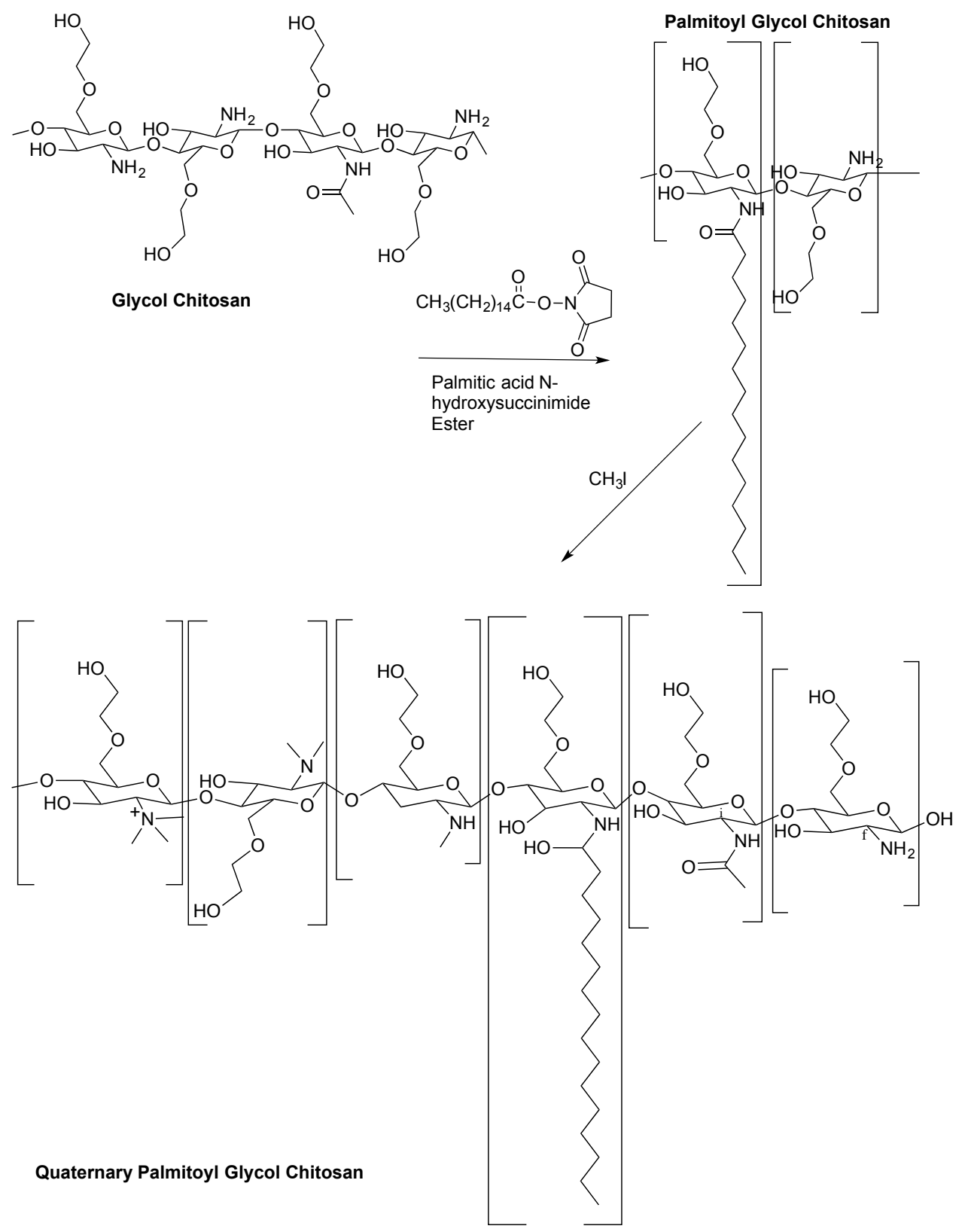

Figure 4.1: Flow diagram of GCPQ synthesis. 


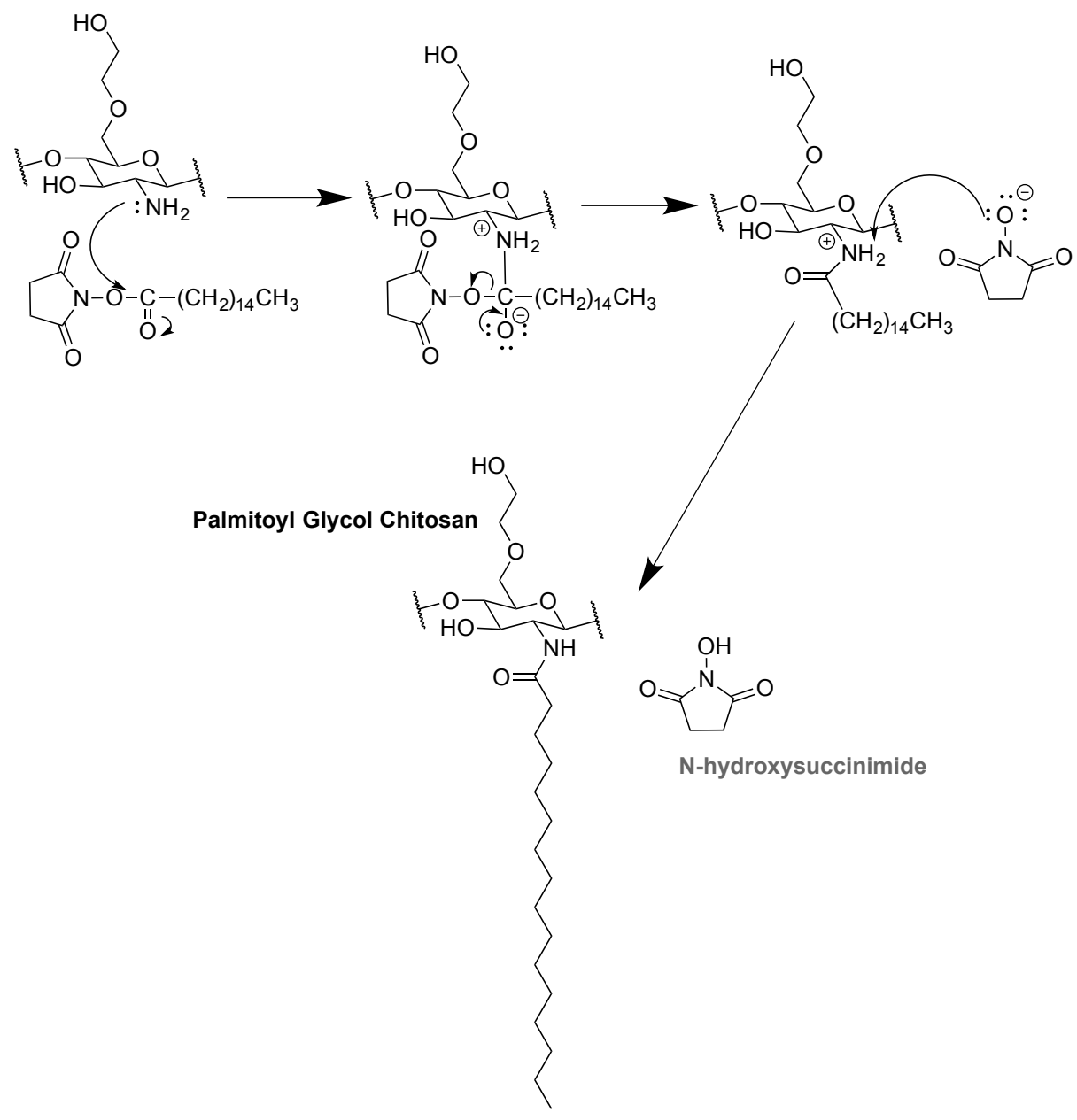

Figure 4.2: Reaction mechanism of palmitoyaltion.

\subsection{Synthesis Method One}

GCPQ was synthesised as previously described in [183, 182, 345]. This method was used to produce the first batch of polymer (051212).

\section{Acid Degradation of GC}

GC (5g) was degraded by dissolving $\mathrm{GC}$ in $\mathrm{HCl}(4 \mathrm{M}, 380 \mathrm{~mL})$ and placed in a preheated water bath at $50^{\circ} \mathrm{C}$ for 48 hours. After 48 hours the reaction was stopped and the product was isolated by dialysis. The dialysis was performed in a membrane with a molecular weight cut off (MWCO) of $3.5 \mathrm{kDa}$ against water $(5 \mathrm{~L})$ for 24 hours with 6 
water changes. Low molecular weight degraded glycol chitosan (dGC) was recovered by freeze drying.

\section{Palmitoylation of dGC}

Palmitoyl glycol chitosan (PGC) was synthesised from dGC in the following manner. A mixture of dGC (500 mg) and sodium bicarbonate $(376 \mathrm{mg})$ was dissolved in a mixture of absolute ethanol (EtOH, $24 \mathrm{~mL}$ ) and double deionised milli-Q water (76mL) to which a solution of palmitic acid N-hydroxysuccinimide (PNS, $792 \mathrm{mg}$ ) dissolved in EtOH $(150 \mathrm{~mL})$ protected from light was added drop wise over 1 hour. The mixture was protected from light and continuously stirred for 72 hours at room temperature.

The product was isolated by evaporating the EtOH using a rotary evaporator. The remaining aqueous phase was extracted with diethyl ether $\left(\mathrm{Et}_{2} \mathrm{O}, 3 \mathrm{x} 100 \mathrm{ml}\right)$. The aqueous mixture of polymer was exhaustively dialysed against water (5L) with 6 changes over 24 hours. The PGC was recovered by freeze drying to give white cotton like solid.

\section{Quaternisation of PGC}

GCPQ was synthesised from PGC in the following manner. PGC (300 mg) was dispersed in N-Methyl-2-pyrrolidone (NMP, $25 \mathrm{~mL}$ ) overnight for 16 hours at room temperature. Sodium hydroxide $(\mathrm{NaOH}, 40 \mathrm{mg})$ dissolved in $\mathrm{EtOH}(4 \mathrm{~mL})$, sodium iodide (NaI, 45mg) and methyl iodide (MeI, $0.44 \mathrm{~mL}$ ) were added and the reaction was stirred under a stream of nitrogen at $36^{\circ} \mathrm{C}$ for 3 hours.

GCPQ was recovered by precipitation with excess $\mathrm{Et}_{2} \mathrm{O}$ and the solid product was washed with copious amounts of $\mathrm{Et}_{2} \mathrm{O}$. The resulting light brown solid was dissolved in water and exhaustively dialysed against water $(5 \mathrm{~L})$ with 6 changes over a period of 24 hours. The quaternary ammonium salt was then passed through a column of Amberlite IRA 96 (5 g) resin that had been washed with $\mathrm{HCl}(150 \mathrm{~mL}, 1 \mathrm{M})$ followed by distilled water $(10 \mathrm{~L})$ until a neutral $\mathrm{pH}$ was obtained. The GCPQ was recovered from the clear elute by freeze drying to give a fibrous solid. 


\subsection{Synthesis Method Two}

The method described below was used to synthesise the remaining batches of polymer. The acid degradation of GC remained the same and was followed as described above in section 4.2.2.1.1. This method was used as it reduced polymer synthesis time from 30 days to 10 days and used lower amount of chemicals, thereby saving money in both resources and time [364]. This was due to a more efficient method of palmitoylation of dGC.

\section{Palmitoylation of dGC}

PGC was synthesised from dGC in the following manner. dGC $(1 \mathrm{~g})$ was dissolved in DMSO $(30 \mathrm{ml})$ and triethylamine $\left(\mathrm{Et}_{3} \mathrm{~N}, 1.15 \mathrm{ml}\right)$ and left to stir for 2 hours. PNS (958 $\mathrm{mg}$ ) was added to the dGC solution and left to stir protected from light for 16 hours at room temperature.

PGC was recovered from the reaction by precipitating the reaction using acetone/ $/ \mathrm{Et}_{2} \mathrm{O}$ $(150 \mathrm{ml}, 1: 2)$. The suspension was stirred gently and left to settle to obtain a precipitate. The majority of the supernatant was syphoned off, the remaining precipitate was washed 3 times with acetone and then 3 times with $\mathrm{Et}_{2} \mathrm{O}$. The remaining product was filtered using a glass sintered filter and left to dry under vacuum. The product was recovered as a brown solid.

\section{Quaternisation of PGC}

GCPQ was synthesised from PGC in the following manner. PGC (1g) was dispersed in NMP (82mL) overnight for 16 hours at room temperature. $\mathrm{NaOH}(135 \mathrm{mg})$ as a fine powder was added to the reaction mixture and left to stir for 45 minutes. NaI (154 $\mathrm{mg}$ ) was added to the reaction mixture and left to stir for 10 minutes under a stream of nitrogen. MeI $(1.5 \mathrm{ml})$ was added to the reaction mixture and the reaction was stirred under a stream of nitrogen at $36^{\circ} \mathrm{C}$ for 3 hours.

GCPQ was recovered by precipitation with excess $\mathrm{Et}_{2} \mathrm{O}$ and the solid product was 
washed with copious amounts of $\mathrm{Et}_{2} \mathrm{O}$. The resulting light brown solid was dissolved in $\mathrm{MeOH}$ and exhaustively dialysed against water $(5 \mathrm{~L})$ with 6 changes over a period of 24 hours. The solution within the dialysis membrane was collected and stirred with Amberlite IRA 140 (5 g) resin for $15 \mathrm{~min}$ at room temperature. The Amberlite was filtered from GCPQ solution under vacuum using a Buchner funnel. The GCPQ was recovered from the clear filtrate by freeze drying to give a fibrous solid.

Removal of iodide by the Amberlite mixture was confirmed using the nitrite test. Sodium nitrite $(200 \mathrm{mg})$ was dissolved in acetic acid (AcOH, 2.5\%, $1 \mathrm{ml})$. GCPQ filtrate solution $(1 \mathrm{ml})$ was added to cholorform $(0.5 \mathrm{ml})$. To the GCPQ biphasic mixture the sodium nitrite solution was added. If the chloroform layer turned pink or violet this indicated the polymer still contained iodide anions. If iodide was detected the GCPQ Amberlite step was repeated and, the filtrate was retested using the nitrite test..

\subsection{NMR Characterisation of Polymers}

NMR analysis of the various products in polymer synthesis were carried out as previously described [182, 345]. In a vial, the respective polymer $(10 \mathrm{mg})$ was dissolved in a suitable solvent $(0.7 \mathrm{~mL})$. dGC dissolved in $\mathrm{D}_{2} \mathrm{O}, \mathrm{PGC}$ in a mixture of $\mathrm{MeOH}-$ $\mathrm{d}_{4}, \mathrm{D}_{2} \mathrm{O}, \mathrm{AcOH}-\mathrm{d}_{6}(40: 20: 5)$ and GCPQ in a mixture of $\mathrm{MeOH}-\mathrm{d}_{4}$ and DCl (70:1). Palmitoylation and quaternisation percentages were calculated from the spectrum by taking the ratio of the respective peaks against sugar proton peaks. The equations for these two calculations are shown below in equation 4.4 and 4.5 .

$$
\text { Palmitoylation }(\%)=\frac{\frac{\delta \text { methyl protons of palmitoyl }}{3 \text { protons }}}{\frac{\delta \text { sugar chain protons }}{9 \text { protons }}} \times 100
$$




\subsection{Molecular Weight Characterisation of Polymer}

The molecular weight of GCPQ was calculated as previously described [182, 183]. GPC experiments were performed using GPC multi angle laser light scattering (GPCMALLS). Measurements were performed using a DAWN HELEOS ${ }^{\circledR}$ II MALLS detector $(\lambda=658 \mathrm{~nm})$, optilab rEX interferometer refractometer (dRI) detector and a Quasi Ecstatic Light scattering (QELS) detector (Wyatt Technology Corporation, USA). Filtered samples of GCPQ $(0.2 \mu \mathrm{m}, 100 \mu \mathrm{L})$ were injected into a POLYSEP ${ }^{\mathrm{TM}}$-GFC-P-400 column (300x7.8 mm, exclusion limit PEG=200 kDa) attached to POLYSEP ${ }^{\text {TM }}-$ GFCP guard column $(35 \times 7.8 \mathrm{~mm}$ ) (Phenomenex, UK) using a Waters 717 Plus auto sampler (Wyatt Technology corporation, USA) with a flow rate of $0.7 \mathrm{ml} \mathrm{min}^{-1}$. The data was analysed using Astra for windows (version 4.90.08) software.

A mixture of $\mathrm{MeOH}$ and acetate buffer (65:35) was used as the mobile phase. Acetate buffer ( $500 \mathrm{mM}$, acetate buffer, $\mathrm{pH} 4)$ was prepared by dissolving anhydrous sodium acetate $(24.6 \mathrm{~g})$ and glacial acetic acid $(11.4 \mathrm{~mL}, 17 \mathrm{M})$ in double deionised water milli-Q water $(1 \mathrm{~L})$. The buffer was filtered using $0.2 \mu \mathrm{M}$ PES filter (MillexHA, Millipore) before passing thought the GPC system. The MeOH was used to help break up the self-aggregated GCPQ micelles in the aqueous media, helping to reduce an overestimation of molecular weight.

The specific refractive index increment $(\mathrm{dn} / \mathrm{dc})$ was measured using an Optilab DSP interferometer (Wyatt Technology Corporation, USA) set at a wavelength of 690 nm. Samples of GCPQ were prepared in their mobile phase as prepared above. Each concentration of polymer was manually injected, the $\mathrm{dn} / \mathrm{dc}$ was calculated using Astra for windows (version 4.90.08) software.

For each polymer two samples were made; one to be used for the MALLS measurement and one for the $\mathrm{dn} / \mathrm{dc}$ measurement. For the MALLS measure a solution of polymer $\left(5 \mathrm{mg} \mathrm{ml}^{-1}\right)$ was made using GCPQ in $\mathrm{MeOH}$ :acetate buffer, then filtered through a PES filter $(0.22 \mu \mathrm{m})$. For the $\mathrm{dn} / \mathrm{dc}$ measurements a number of polymer solutions ranging from $0.1-0.6 \mathrm{mg} \mathrm{ml}^{-1}$ were made; from which the refractive index of the individual polymer concentration was measured. 


\subsubsection{Formulation Development}

Two different methods were used to develop a formulation for METx. The first involved probe sonication of a mixture of METx and GCPQ, this is referred to as the METx:GCPQ formulations. The second method involved using glycerol as a cosolvent which is referred to as GCPQ (glycerol) method.

\subsection{GCPQ Formulations}

A mixture of GCPQ and METx (1 mg) dispersed in water (MilliQ, $1 \mathrm{~mL}$ ) was vortexed followed by probe sonication using a QSonica Sonicator with a micro tip (Cole Parmer Instruments Co. Ltd, UK) for 15 minutes on ice, with a cycle of 5 minutes on and 3 minutes off. The instrument was set at $30 \%$ of its maximum output. The weight of GCPQ was varied according to the drug:polymer ratio required.

\subsection{GCPQ Glycerol Formulations}

A mixture of GCPQ and METx (1 mg) was dispersed in glacial acetic acid (1mL, $17 \mathrm{M})$ and vortexed. To this mixture glycerol solution (10\%) was added to produce final glycerol concentration (1\%) and vortexed. This was followed by probe sonication using a Qsonica Sonicator with a micro tip (Cole Parmer Instruments Co. Ltd, UK) for 15 minutes on ice, with a cycle of 5 minutes on and 3 minutes off. The formulation was frozen and freeze dried (Martin Christ Freeze Dryer, Germany). The cake was the resuspended in dextrose (5\%). The weight of GCPQ was varied according to the drug:polymer ratio required. 


\subsubsection{Formulation Characterisation}

\subsection{Transmission Electron Microscopy}

TEM was performed by placement of a drop of the nanoparticle formulation on a Formvar/carbon coated grid. Excess sample was blotted off with Whatman No. 1 filter paper and the sample was negatively stained with uranyl acetate $(1 \% \mathrm{w} / \mathrm{v})$. Samples were imaged using a CM120 BioTwin Transmission Electron Microscope (Philips, USA). Images were captured using an AMT digital camera (Woburn, USA).

\subsection{Nanoparticle Size}

GCPQ and GCPQ (glycerol) formulations were prepared as described in section 4.2.2.2.1 and 4.2.2.2.2 respectively. Samples were left for 15 minutes to cool before particle sizing was carried out using DLS and a Nanozetasizer ZS (Malvern Instruments, UK) at $25^{\circ} \mathrm{C}$, wavelength $633 \mathrm{~nm}$ and detection angle of $90^{\circ}$. Data was obtained and analysed using Zeta Software (version 7.01 for windows) software.

\subsection{Role of Glycerol}

To understand the role of acetic acid, glycerol and GCPQ within the METx:GCPQ (glycerol) formulation, the impact of each of the components was studied. Formulations were made with either one or two of the components excluded. The first formulations were made with either glycerol, GCPQ or acetic acid missing. Next formulations were made with a combination of glycerol, GCPQ or acetic acid missing. Each control formulation was analysed visually looking for the presence of precipitation. 


\subsubsection{In Vitro Stability}

\subsection{Plasma Stability}

Male Sprague Dawley rats ( $\mathrm{n}=3$ ) weighing 200-250 g (Charles-River, UK) were habituated for 1 week before they were euthanised using an overdose of carbon dioxide. An incision was made through the abdominal wall and the sternum was cut open to expose the heart. Slowly a needle was inserted into heart and the blood from the rat was collected by negative pressure on the needle. Blood was collected in a vacutainer coated with EDTA and kept on ice $\left(4^{\circ} \mathrm{C}\right)$ until centrifugation. Plasma was obtained as a supernatant after centrifugation of plasma for $10 \mathrm{~min}$ at $2000 \mathrm{~g}$ and $4^{\circ} \mathrm{C}$ (Hermle z323k refrigerated centrifuge, HERMLE Labortechnik, Germany). The separated rat plasma was stored at $-20{ }^{\circ} \mathrm{C}$ until required.

Before each plasma stability experiment the plasma was thawed just prior to the experiment. Plasma was diluted to 30 or $10 \%$ (v/v) using saline $\mathrm{NaCl}(0.9 \%)$. METx $\left(7.2 \times 10^{-4} \mathrm{M}, 50 \mu \mathrm{L}\right)$ was suspended in diluted plasma $(450 \mu \mathrm{L})$ that had been allowed to warm for 30 minutes at $37^{\circ} \mathrm{C}$.

GCPQ and GCPQ (glycerol) formulations were prepared as described in section 4.2.2.2.1 and 4.2.2.2.2 respectively. METx dissolved in DMSO was also prepared. Each formulation or solution $(50 \mu \mathrm{L})$ was suspended in diluted plasma (450 $\mu \mathrm{L})$ at $37^{\circ} \mathrm{C}$, and shaken at $130 \mathrm{rpm}$ for a maximum of 4 hours.

At regular time intervals aliquots $(50 \mu \mathrm{L})$ were removed and mixed with DMF (150 $\mu \mathrm{L})$. The DMF helped to precipitate the plasma and the solvent used to dissolve the nanoparticles allowing METx to be released for analysis. Samples were then immediately vortexed and centrifuged for 10 minutes at 13,000 rpm (MSE micro centaur centrifuge, MSE, UK). The supernatant $(100 \mu \mathrm{L})$ was collected and $40 \mu \mathrm{L}$ was analysed by reverse HPLC in section 2.3. Each formulation was tested in triplicate. 


\subsection{Gastric Stability}

Simulated gastric fluid (SGF) was prepared according to the British Pharmacopeia [245]. SGF was prepared by dissolving sodium chloride ( $\mathrm{NaCl}, 2 \mathrm{~g})$ and pepsin $(3.2 \mathrm{~g})$ in double deionised milliQ water, after which $\mathrm{HCl}(1 \mathrm{M}, 80 \mathrm{~mL})$ was added and made up to $1000 \mathrm{~mL}(\mathrm{pH} 1.2)$. METx $\left(1.8 \times 10^{-4} \mathrm{M}, 100 \mu \mathrm{L}\right)$ was suspended in SGF $(900 \mu \mathrm{L})$ that had been allowed to warm for 15 minutes at $37^{\circ} \mathrm{C}$.

METx:GCPQ formulations were prepared as described in section 4.2.2.2.1. METx dissolved in DMF was also prepared, by dissolving METx in DMF followed by vortexing. Each formulation or solution $(100 \mu \mathrm{L})$ was suspended in SGF $(900 \mu \mathrm{L})$ at $37^{\circ} \mathrm{C}$, and shaken at $130 \mathrm{rpm}$ for a maximum of 2 hours.

At regular time intervals aliquots $(100 \mu \mathrm{L})$ were removed and mixed with sodium carbonate $(2 \mathrm{M}, 10 \mu \mathrm{L})$ to stop digestion and DMF $(100 \mu \mathrm{L})$ was added to destroy the nanoparticles and solubilise METx. The same amount of peptide was added to a mixture of sodium carbonate (2M): SGF (1:10) to serve as control. DMF samples were centrifuged for 10 minutes at 13,000rpm. The supernatant $(100 \mu \mathrm{L})$ was collected and analysed by HPLC as described in section 2.3. Each formulation was tested in triplicate.

\subsection{Intestinal Stability}

Intestinal stability was tested using rat intestinal wash (RIW). Male Sprague Dawley rats $(\mathrm{n}=3)$ weighing 200-250 g (Charles-River, UK) were habituated for 1 week and fasted for 16 hours before they were euthanised using an overdose of carbon dioxide. An abdominal incision was made to expose the intestine and around $30 \mathrm{~cm}$ of the intestine was removed starting from the duodenum. The lumen of each intestine segment was immediately flushed with ice cold PBS buffer $(10 \mathrm{~mL}, 50 \mathrm{mM}, \mathrm{pH}$ 6.6). The washings from all the intestinal segments were pooled and centrifuged at 4,000 rpm for 20 minutes at $4^{\circ} \mathrm{C}$ immediately using Hermle z323k refrigerated centrifuge (HERMLE Labortechnik, Germany). The supernatant was stored at $-20^{\circ} \mathrm{C}$ until used. 
The protein content of RIW was determined using the Bradford Assay [365] and diluted to $40 \mu \mathrm{g} \mathrm{mL}^{-1}$ total protein using PBS. BSA was used as the standard. The calibration curve was produced for the range of $0.1-1.4 \mathrm{mg} \mathrm{ml}^{-1}$ and measured at 595nm using the UV Shimadzu (Shimadzu Corporation, Japan). METx $\left(1.8 \times 10^{-4} \mathrm{M}\right.$, $150 \mu \mathrm{L})$ was suspended in diluted RIW (1350uL, diluted to $40 \mu \mathrm{g} \mathrm{ml}^{-1}$ total protein) that has been allowed to warm for 15 minutes at $37^{\circ} \mathrm{C}$.

GCPQ formulations were prepared as described in section 4.2.2.2.1. METx dissolved in DMF was also prepared, by dissolving METx in DMF. Each formulation or solution $(150 \mu \mathrm{L})$ was suspended in RIW $(1350 \mathrm{uL})$ at $37^{\circ} \mathrm{C}$, and shaken at $130 \mathrm{rpm}$ for a maximum of 4 hours.

At regular time intervals aliquots $(150 \mu \mathrm{L})$ were removed and mixed with glacial acetic acid (17M, $10 \mu \mathrm{L})$ to stop digestion and DMF $(150 \mu \mathrm{L})$ was added to solubilise the peptide and destroy the nanoparticles allowing METx to be released for analysis. The DMF samples were centrifuged for 10 minutes at 13,000 rpm. The same amount of peptide was added to a mixture of acetic acid (17M): RIW (1:15) to serve as a control. The supernatant $(100 \mu \mathrm{L})$ was collected and analysed by HPLC as described in section 2.3. Each formulation was tested in triplicate.

\subsection{Shelf Life Stability}

Formulations were prepared using the METx:GCPQ (glycerol) method as described in section 4.2.2.2.2. A bulk volume of the formulation was prepared and aliquoted into individual vials. The individual vials were freeze dried at the same time and resuspended on the the given days. On the day of testing; the aliquot was resuspended in dextrose $(5 \%)$. The freeze dried cakes were stored at room temperature and in the fridge $\left(4^{\circ} \mathrm{C}\right)$ and on days $1,2,4,8$ and 14 were resuspended in dextrose $(5 \%)$. The formulation was analysed by HPLC and DLS for drug content and size respectively. 


\subsection{Statistical Analysis}

For comparing the means of different groups, one-way ANOVA test (IBM SPSS) with a Bonferroni's post hoc test (IBM SPSS) was performed. In each case, for the results to be considered as significantly different, a p-value of less than 0.05 was set. The Student's t test was used to assess any statistical significant differences among the means of two groups. 


\subsection{Results}

\subsubsection{Polymer Synthesis}

\subsubsection{NMR}

\subsection{Degradation of GC}

GC was degraded for 48 hours at $50^{\circ} \mathrm{C}$ in $\mathrm{HCl}$, dGC was characterised by NMR in $\mathrm{D}_{2} \mathrm{O}$. The NMR spectrum is shown in figure 4.3. The NMR peaks of dGC was assigned as previously described in [182, 345], the assignments are shown in table 4.3. The NMR spectrum for $\mathrm{dGC}$ is shown in figure 4.3 .

Table 4.3: Chemical shift assignment of dGC NMR.

\begin{tabular}{cc}
\hline$\delta(\mathrm{ppm})$ & Chemical Environment \\
\hline 1.9 & $\mathrm{CH}_{3}-\mathrm{CO}-\mathrm{NH}-$, acetylated glycol chitosan \\
2.9 & $-\mathrm{CH}-\mathrm{CH}-\mathrm{NH}-\mathrm{CO}-$, glycol chitosan \\
$3.5-4.5$ & $-\mathrm{CH}(\mathrm{OH})-$ and $-\mathrm{CH}_{2}-\mathrm{OH}$, glycol chitosan \\
4.7 & water \\
5.3 & O-CH-O anomeric proton \\
\hline
\end{tabular}




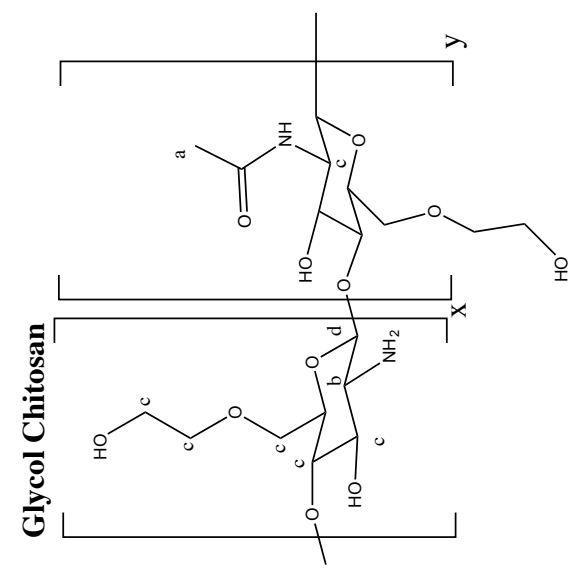

$\mathrm{O}^{2} \mathrm{H}$

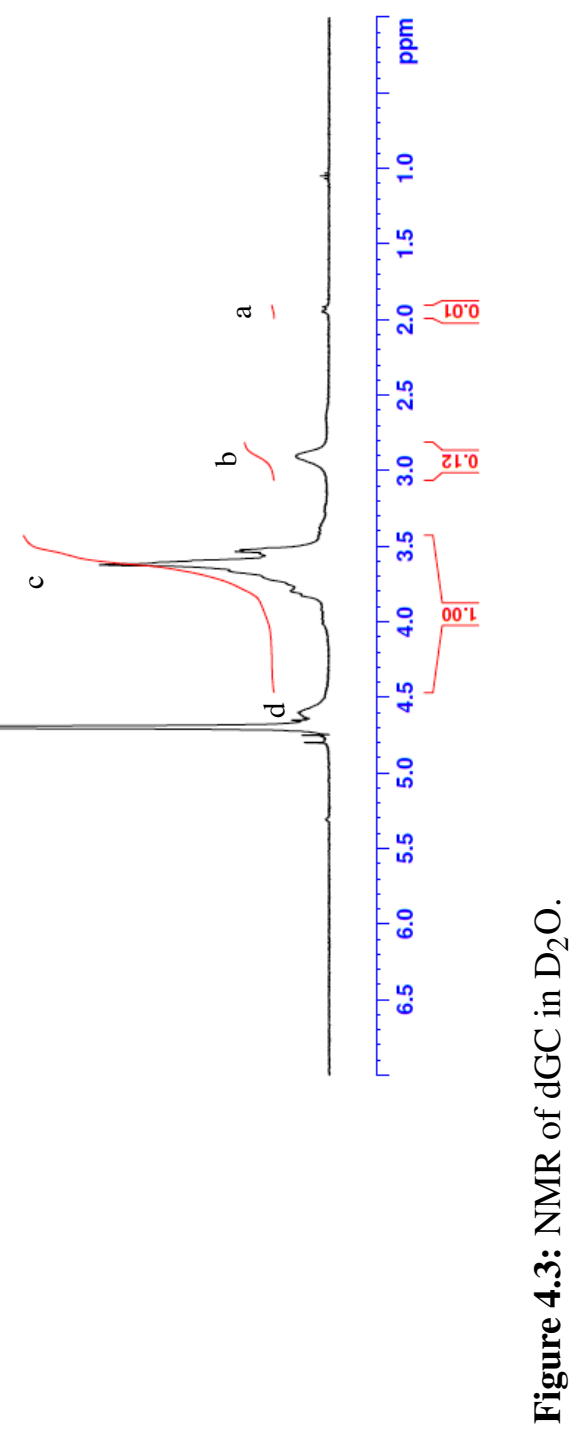




\subsection{Palmitoylation of dGC}

dGC was palmitoylated using PNS. PNS is an activated ester of palmitic acid, that is able to react with the amine group of glycol chitosan more easily than palmitic acid alone. PGC was characterised using NMR with a mixture of $\mathrm{MeOH}-\mathrm{d}_{4}, \mathrm{D}_{2} \mathrm{O}$ and AcOD (40:20:5). The NMR spectrum for PGC is shown in figure 4.4. The assignment of the NMR peaks is shown in table 4.4 .

Table 4.4: Chemical shift assignment of PGC NMR.

\begin{tabular}{cc}
\hline$\delta(\mathrm{ppm})$ & Chemical Environment \\
\hline 0.9 & $\mathrm{CH}_{3}-\left(\mathrm{CH}_{2}\right)_{14}-\mathrm{CO}-$, palmitoyl \\
1.4 & $\mathrm{CH}_{3}-\left(\mathrm{CH}_{2}\right)_{12}-\mathrm{CH}_{2}-\mathrm{CH}_{2}-\mathrm{CO}-$, palmitoyl \\
1.6 & $\mathrm{CH}_{3}-\left(\mathrm{CH}_{2}\right)_{12}-\mathrm{CH}_{2}-\mathrm{CH}_{2}-\mathrm{CO}-$, palmitoyl \\
2.0 & Acetic Acid \\
2.4 & $\mathrm{CH}_{3}-\mathrm{CO}-\mathrm{NH}-$, acetylated glycol chitosan \\
3.1 & $-\mathrm{CH}-\mathrm{CH}-\mathrm{NH}-\mathrm{CO}-$, glycol chitosan \\
3.3 & Methanol \\
$3.5-4.5$ & $-\mathrm{CH}(\mathrm{OH})-$, glycol chitosan \\
& $-\mathrm{CH}_{2}-\mathrm{OH}$, glycol chitosan \\
5.1 & water \\
\hline
\end{tabular}




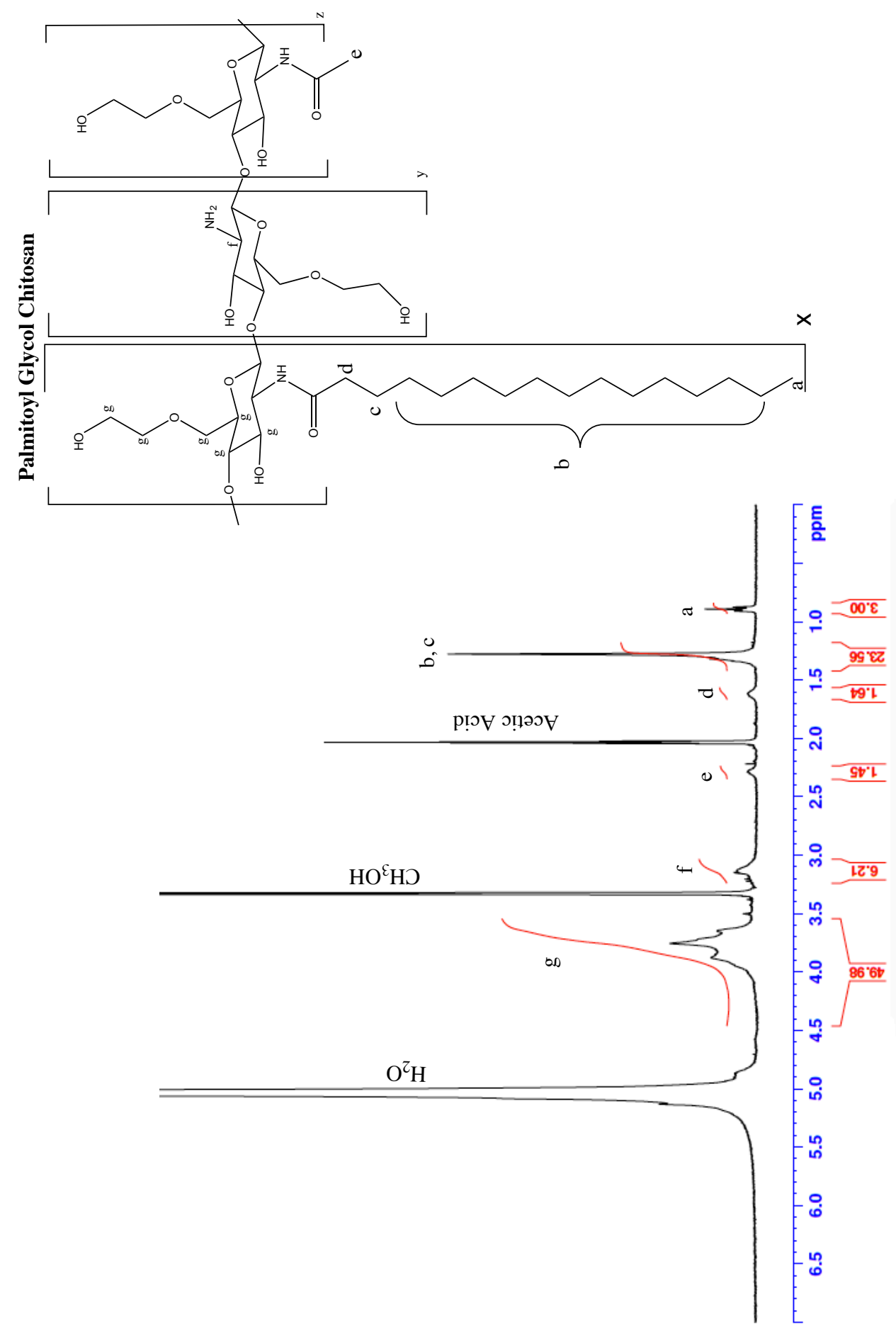

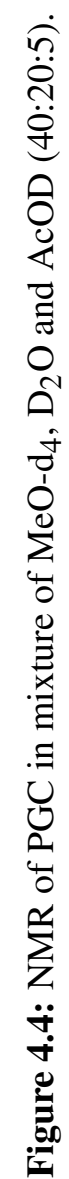




\subsection{Methylation of PGC}

PGC was methylated using MeI. The GCPQ was characterised using NMR and GPC. GCPQ was characterised using NMR in $\mathrm{CD}_{3} \mathrm{OD}$ and $\mathrm{DCl}$ (70:1). Figure 4.5 shows a NMR of GCPQ, table 4.5 shows the peak assignments of the NMR. Using NMR the palmitoylation percentage $(\mathrm{P} \%)$ and quaternization percentage $(\mathrm{Q} \%)$ were calculated using equation 4.4 and 4.5 respectively, these are reported in table 4.6 .

Table 4.5: Chemical shift assignment of GCPQ NMR.

\begin{tabular}{cc}
\hline$\delta(\mathrm{ppm})$ & Chemical Environment \\
\hline 0.9 & $\mathrm{CH}_{\mathbf{3}}-\left(\mathrm{CH}_{2}\right)_{14}-\mathrm{CO}-$, palmitoyl \\
1.4 & $\mathrm{CH}_{3}-\left(\mathrm{CH}_{2}\right)_{12}-\mathrm{CH}_{2}-\mathrm{CH}_{2}-\mathrm{CO}-$, palmitoyl \\
1.6 & $\mathrm{CH}_{3}-\left(\mathrm{CH}_{2}\right)_{12}-\mathrm{CH}_{2}-\mathrm{CH}_{2}-\mathrm{CO}-$, palmitoyl \\
2.4 & $\mathrm{CH}_{3}-\mathrm{CO}-\mathrm{NH}-$, acetylated glycol chitosan \\
2.9 & $-\mathrm{CH}-\mathrm{CH}-\mathrm{NH}-\mathrm{CO}-$, glycol chitosan \\
$3.0-3.2$ & $-\mathrm{CH}-\mathrm{CH}-\mathrm{NH}-\mathrm{CH}_{\mathbf{3}}$ \\
& $-\mathrm{CH}-\mathrm{CH}-\mathrm{N}\left(\mathrm{CH}_{\mathbf{3}}\right)_{\mathbf{2}}$ \\
3.3 & Methanol \\
3.4 & $\mathrm{CH}-\mathrm{CH}-\mathrm{N}(\mathrm{CH})_{\mathbf{3}}$ \\
$3.5-4.5$ & $-\mathrm{CH}(\mathrm{OH})-$, glycol chitosan \\
& $-\mathrm{CH}-\mathrm{OH}$, glycol chitosan \\
5.7 & Water \\
\hline
\end{tabular}




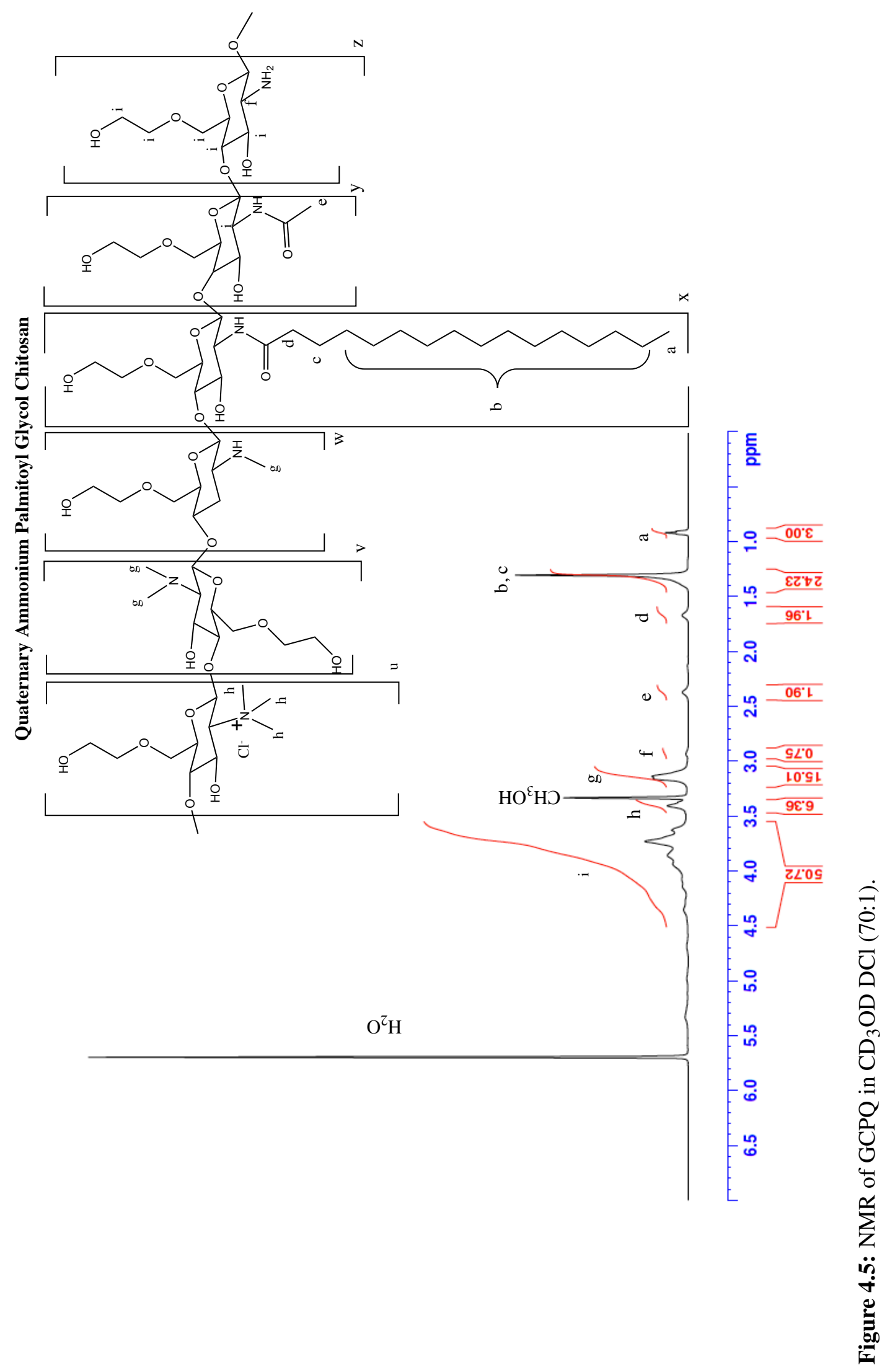




\subsection{GPC}

The molecular weight and polydispersity (PDI) of GCPQ was measured using size exclusion chromatography. A mixture of $\mathrm{MeOH}$ :acetate buffer (65:35) was used to ensure the measured molecular weight was of the polymer itself and not micelles. The molecular weights for each of the GCPQs are shown in table 4.6. The average molecular weight was $8.4 \mathrm{KDa}$ with a PDI of 1.12 .

\subsection{Summary of GCPQ}

Various batches of GCPQ were synthesised and analysed using ${ }^{1} \mathrm{H}$ NMR and GPC. The molecular weight, PDI of molecular weight, palmitoylation and quarternisation of each batch of GCPQ is shown in table 4.6. The average molecular weight of the GCPQ was $10.1 \mathrm{kDa}$ with a PDI of 1.14 . The average palmitoylation was $21 \%$ and the average percentage of quaternization was $11 \%$. Batch 051212 was made using the first method of GCPQ synthesis, the remainder of the GCPQ batches were made using the second method of GCPQ synthesis. Table 4.6 also shows the palmitoylation and quaternization of each batch of GCPQ. There is a wide range in the $\mathrm{M}_{\mathrm{W}}$ ranging from 6.2-18.6 kDa, however the PDI remains close to 1 .

Table 4.6: Molecular weights of GCPQ measured by GPC.

\begin{tabular}{|c|c|c|c|c|c|}
\hline \multirow[t]{2}{*}{ Batch } & \multicolumn{3}{|c|}{ Molecular Weight (KDa) } & \multirow{2}{*}{$\begin{array}{c}\text { Palmitoylation } \\
(\%)\end{array}$} & \multirow{2}{*}{$\begin{array}{c}\text { Quaternization } \\
(\%)\end{array}$} \\
\hline & Mw & $\mathrm{Mn}$ & Mw/Mn & & \\
\hline \multicolumn{6}{|c|}{ Synthesis Method 1} \\
\hline 051212 & 7.12 & 7.01 & 1.004 & 25.4 & 11.6 \\
\hline \multicolumn{6}{|c|}{ Synthesis Method 2} \\
\hline 121114 & 6.20 & 5.59 & 1.109 & 19.9 & 13.1 \\
\hline 210115 & 11.87 & 9.21 & 1.289 & 18.0 & 12.9 \\
\hline 150615 & 7.19 & 6.29 & 1.143 & 21.5 & 9.4 \\
\hline 090315 & 18.6 & 14.8 & 1.257 & 18.7 & 11.7 \\
\hline 050815 & 9.60 & 9.23 & 1.040 & 20.7 & 9.5 \\
\hline Average & 10.10 & 8.70 & 1.14 & 20.7 & 11.3 \\
\hline SD & 4.25 & 3.05 & 0.10 & 2.4 & 1.5 \\
\hline
\end{tabular}




\subsubsection{Formulation Development}

Formulations were made using two methods. The first method was probe sonication of METx and GCPQ suspended in water. The second method used glycerol as a cosolvent. Both formulations were analysed using DLS and TEM for size and tested for stability.

\subsubsection{METx:GCPQ Formulations}

\subsection{Transmission Electron Microscopy Images}

Morphological characterisation of the different METx:GCPQ formulations was performed using TEM. Each of the different formulations were viewed from two different magnifications. Each of the formulations was freshly prepared prior to TEM imaging and stained few min before imaging.

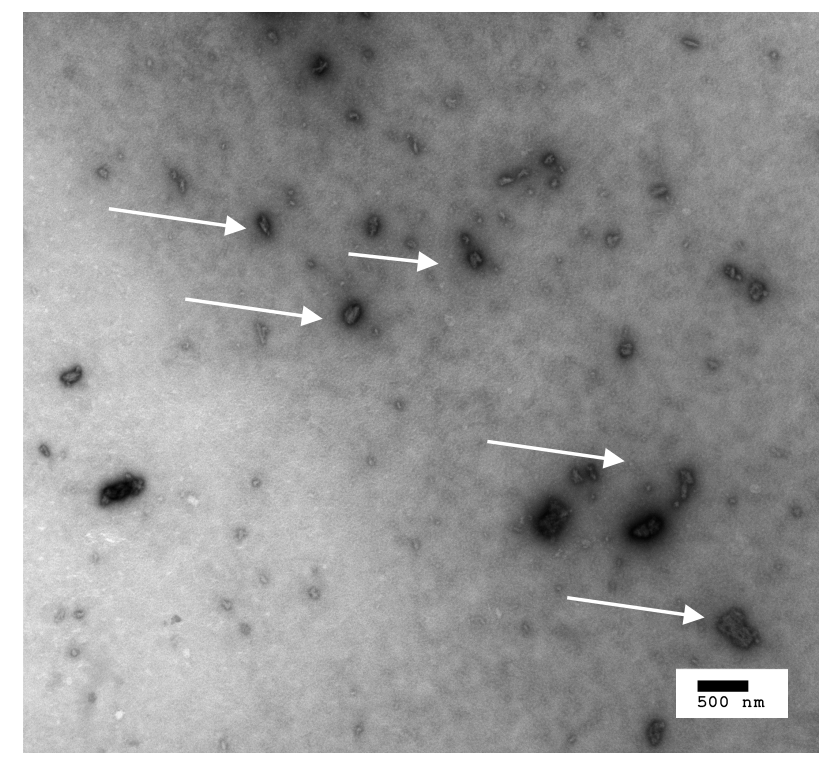

Figure 4.6: Negatively stained TEM images of METx:GCPQ (1:4), white arrows point to fibrous structures, (GCPQ batch: 051212 METx concentration $1 \mathrm{mg} \mathrm{ml}^{-1}$ ).

TEM images of METx suspended and probe sonicated in water are show in figure 2.3. Introduction of GCPQ to METx results in a decreased amount of fibrous structures being seen and generation of micelles, this can be seen in figure 4.6 to 4.8 . All the formulations produced micelles that were within the nanometer size, these micelles 



Figure 4.7: Negatively stained TEM images of METx:GCPQ (1:5), white arrows point to fibrous structures, (GCPQ batch: 051212. METx concentration $1 \mathrm{mg} \mathrm{ml}^{-1}$ ).

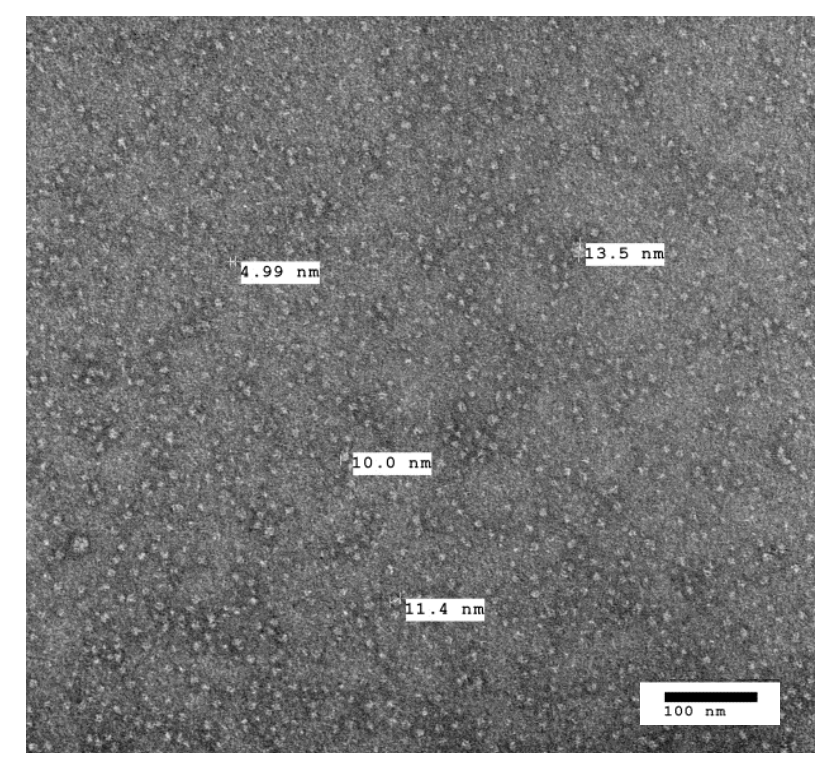

Figure 4.8: Negatively stained TEM images of METx:GCPQ (1:10) (GCPQ batch: 051212, METx concentration $1 \mathrm{mg} \mathrm{ml}^{-1}$ ).

had a spherical shape. It can be seen that as the concentration of GCPQ increases the presence of fibres decreases, the presences of fibrous material is shown by areas in figure 4.6 and 4.7. In figure 4.6 and 4.7. METx:GCPQ (1:4) and (1:5) respectively it can be seen that the size and amount of these fibrous structures is decreasing. Whereas in the lower magnification image of METx:GCPQ (1:10), figure 4.8, there is almost no presence of the fibrous structures. This suggests that GCPQ encapsulates METx, taking it into the hydrophobic core created by the palmitoyl branches of GCPQ in the GCPQ micelles. 


\subsection{Nanoparticle Size}

Freshly prepared METx:GCPQ formulations were measured using DLS the size by intensity distribution is shown in in figure 4.9 and table 4.7 and 4.8 .

\section{Nanoparticle Particle Size by Intensity: METx:GCPQ}

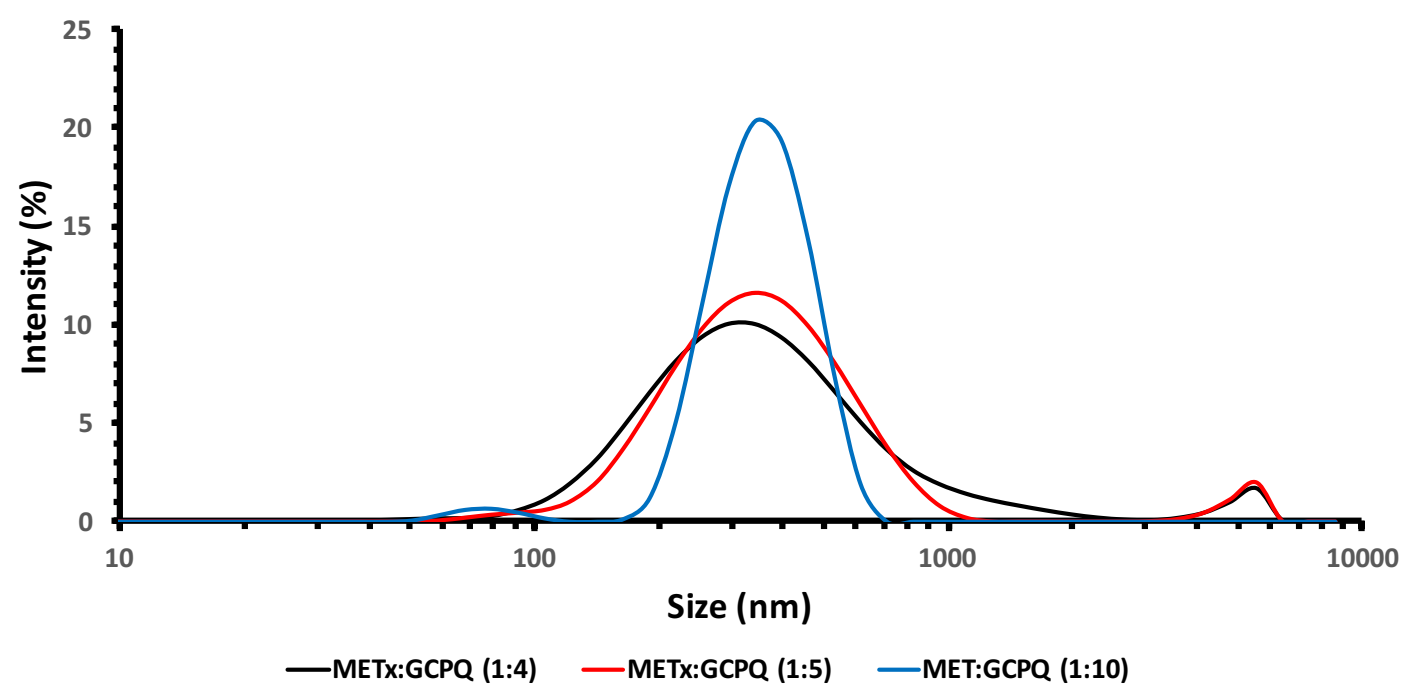

Figure 4.9: DLS intensity distribution graph of METx:GCPQ formulations, (GCPQ batch: 051212, METx concentration $1 \mathrm{mg} \mathrm{ml}^{-1}$ ).

Table 4.7: DLS Peak Size and Population of METx:GCPQ formulations, (GCPQ batch: 051212, METx concentration $1 \mathrm{mg} \mathrm{ml}^{-1}$ ).

\begin{tabular}{ccccc}
\hline Formulation & \multicolumn{3}{c}{ Peak Size Population } \\
\cline { 2 - 5 } & \multicolumn{2}{c}{ Peak 1 } & \multicolumn{2}{c}{ Peak 2 } \\
\cline { 2 - 5 } & Size (nm) & Population (\%) & Size (nm) & Population (\%) \\
\hline $1: 4$ & 410.0 & 96.6 & 5055 & 3.1 \\
$1: 5$ & 373.2 & 96.3 & 5079 & 3.7 \\
$1: 10$ & 363.0 & 97.3 & 76.9 & 2.3 \\
\hline
\end{tabular}

Table 4.8: DLS Z-average, PDI and volume mean sizes of METx:GCPQ formulations, (GCPQ batch: 051212, METx concentration $1 \mathrm{mg} \mathrm{ml}^{-1}$ ).

\begin{tabular}{cccc}
\hline $\begin{array}{c}\text { METx:GCPQ } \\
\text { Formulation }\end{array}$ & $\begin{array}{c}\text { Z-average }(\mathrm{nm}) \\
( \pm \mathrm{SD})\end{array}$ & $\begin{array}{c}\text { PDI } \\
( \pm \mathrm{SD})\end{array}$ & $\begin{array}{c}\text { Volume mean }(\mathrm{nm}) \\
( \pm \mathrm{SD})\end{array}$ \\
\hline $1: 4$ & $323.5(13.25)$ & $0.36(0.04)$ & $941.3(148)$ \\
$1: 5$ & $333.6(5.82)$ & $0.36(0.03)$ & $890.8(141)$ \\
$1: 10$ & $416.7(7.03)$ & $0.43(0.04)$ & $357.6(24)$ \\
\hline
\end{tabular}


The DLS size by intensity of each of the METx:GCPQ formulations is shown in figure 4.9, from which it can be seen that each ratio of MET:GCPQ produced particles within the nanometer range. However, all of the formulations except MET:GCPQ (1:10) also had particles above the nanometer range up to 10 micron in size. When comparing the population percentage sizes of each formulation from table 4.7, it can be seen that majority of particles from each formulation are in the nanometer range. Over $95 \%$ of particles are in the nanometer range. The point of each peak is very similar to each other range from 360 to $410 \mathrm{~nm}$. The ratio METx:GCPQ (1:10) shows no particles within the micron range, however has a small percent of it's population in the sub $100 \mathrm{~nm}$ range. As the ratio of GCPQ increases the z-average size of the particles increases as well, however the PDI stay close to the same. When comparing the volume size of the formulations there is a trend of decreasing size by volume.

\subsection{METx:GCPQ (Glycerol) Formulations}

METx:GCPQ formulations were prepared by dissolving METx and GCPQ in acetic acid and glycerol. The acetic acid was removed by lyophilisation and the cake was reconstituted in dextrose $(5 \%)$.

\section{TEM Images}

Morphological characterisation of the different METx:GCPQ (glycerol) formulations was performed using TEM. Each of the different formulations were viewed from two different magnifications. Each of the formulations was freshly reconstituted prior to TEM imaging and stained few min before imaging.

The TEM images of METx:GCPQ (glycerol) formulations are shown in figure 4.10 to 4.12. METx:GCPQ (glycerol) formulations were prepared with three different ratios of METx:GCPQ 1:1, 1:5 and 1:10. As the ratio of GCPQ increased the presence of fibrous like structures was reduced, however they did not completely disappear as they could still be seen in figure 4.12B, METx:GCPQ (glycerol) (1:10). The micelles that were generated had a size of between $10-50 \mathrm{~nm}$ and were spherical in shape. 

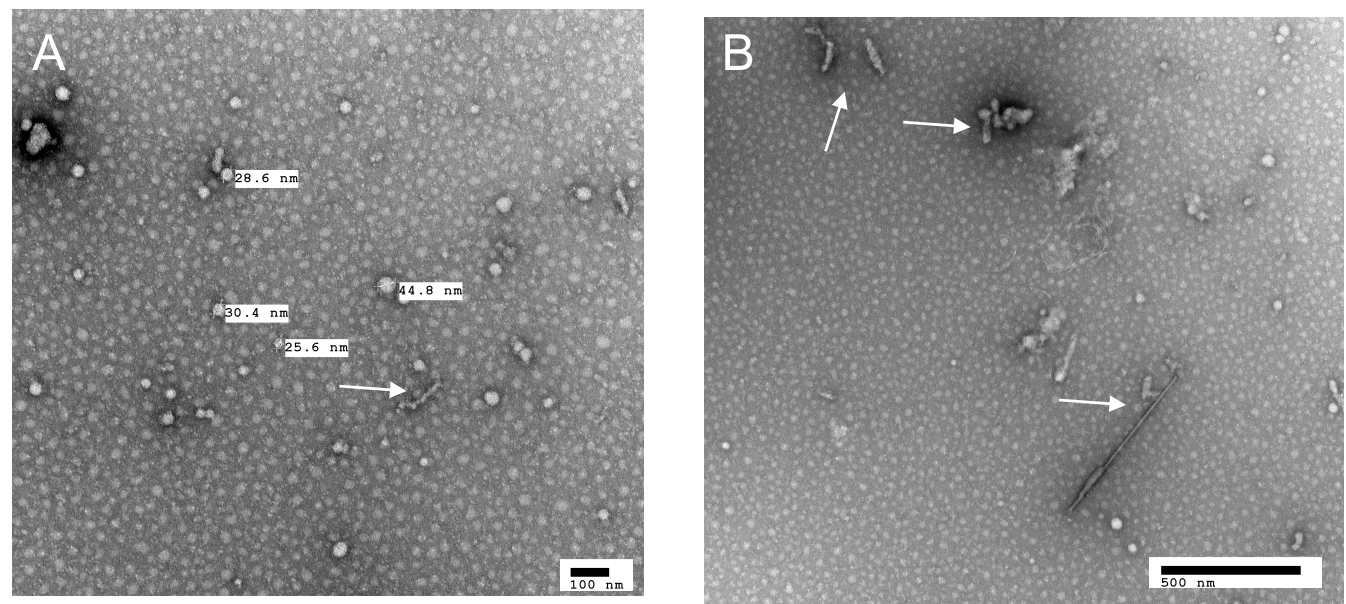

Figure 4.10: Negatively stained TEM images of METx:GCPQ (Glycerol) (1:1), white arrows show presence of fibrous material, (GCPQ batch: 210115, METx concentration $1 \mathrm{mg} \mathrm{ml}^{-1}$ ).
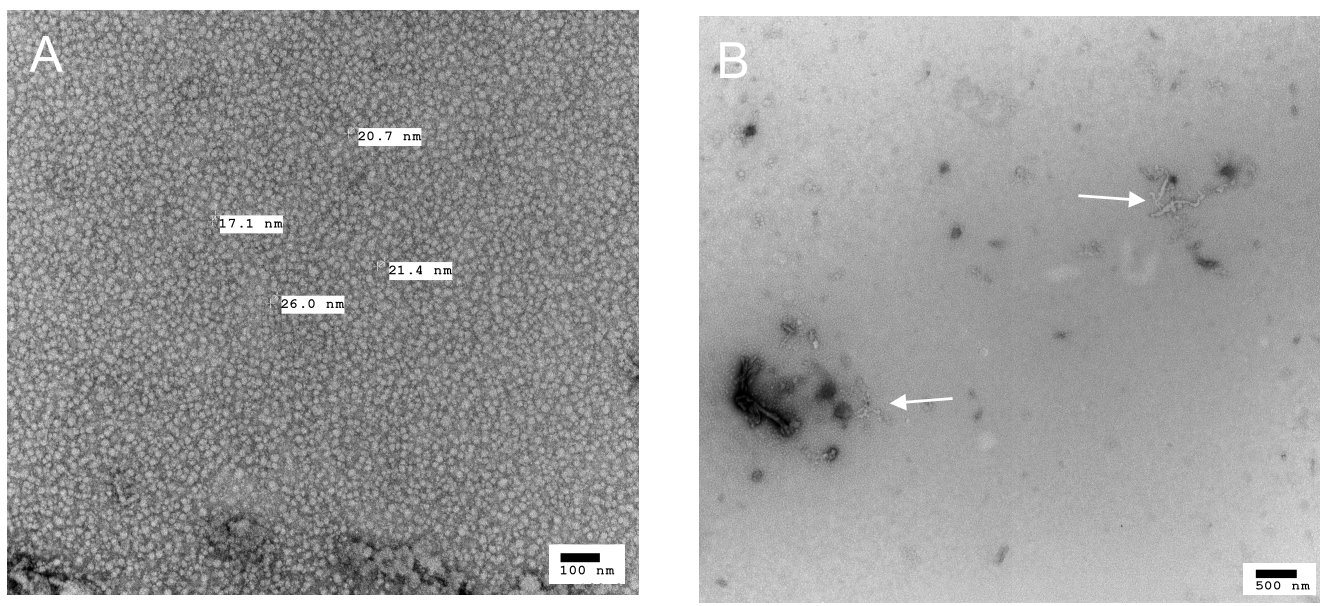

Figure 4.11: Negatively stained TEM images of METx:GCPQ (Glycerol) (1:5), white arrows show presence of fibrous material, (GCPQ batch: 210115, METx concentration $1 \mathrm{mg} \mathrm{ml}^{-1}$ ).
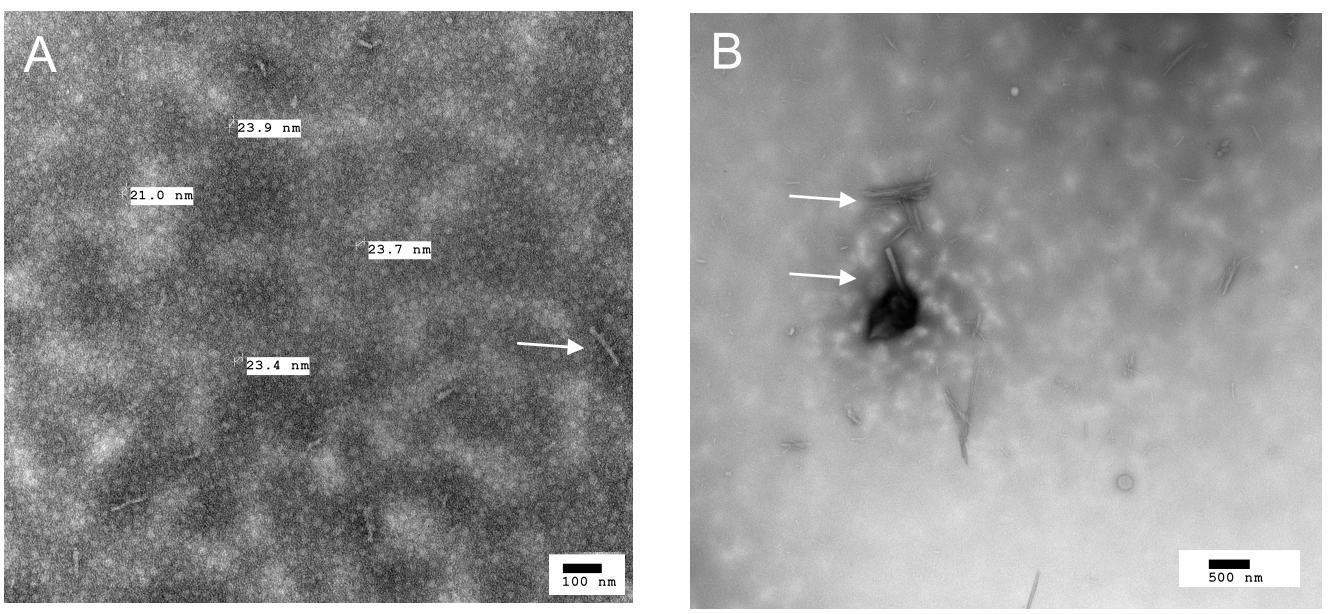

Figure 4.12: Negatively stained TEM images of METx:GCPQ (Glycerol) (1:10), white arrows show presence of fibrous material, (GCPQ batch: 210115, METx concentration 1mg $\left.\mathrm{ml}^{-1}\right)$. 


\section{Nanoparticle Size}

Freshly reconstituted METx:GCPQ (glycerol) formulations were measured using DLS the size by intensity distribution is show in in figure 4.13

\section{Nanoparticle Particle Size by Intensity: METx: GCPQ (Glycerol)}

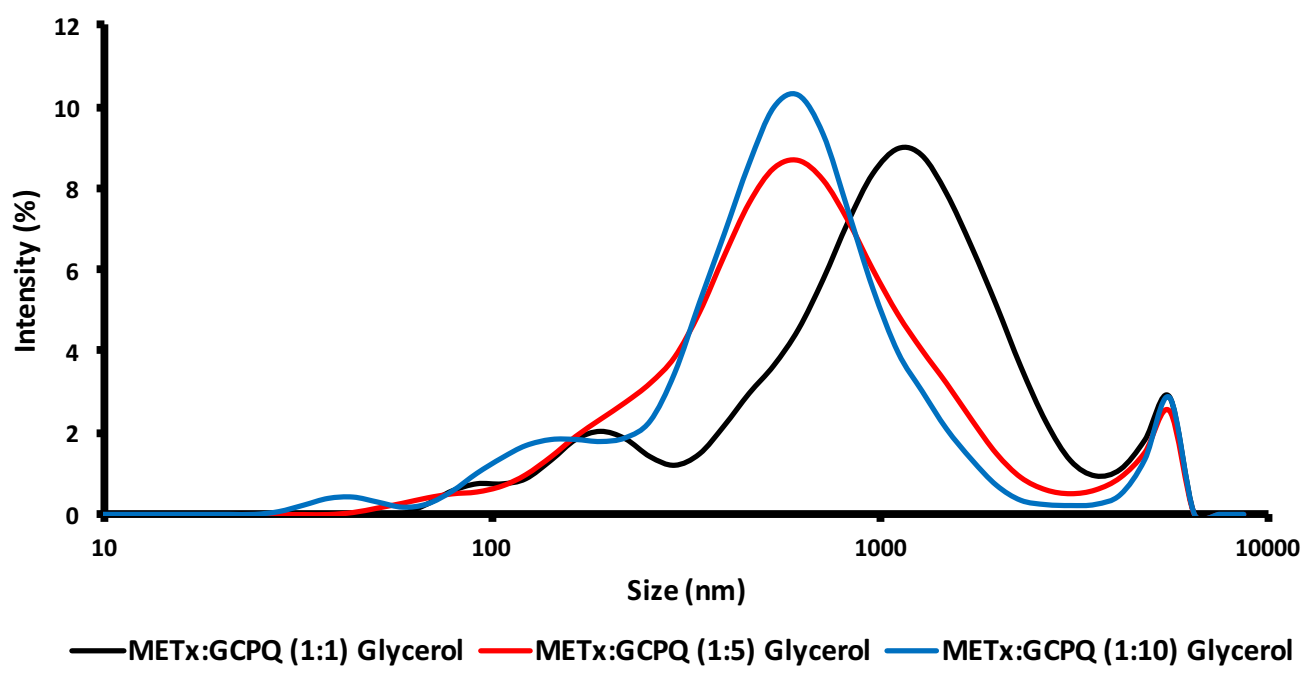

Figure 4.13: $r$ METx:GCPQ (glycerol) formulations, (GCPQ batch: 210115, METx concentration $1 \mathrm{mg} \mathrm{ml}^{-1}$ ).

Table 4.9: DLS Peak Sizes and Population Percent of METx:GCPQ (glycerol) formulations, (GCPQ batch: 210115, METx concentration $1 \mathrm{mg} \mathrm{ml}^{-1}$ ).

\begin{tabular}{ccccccc}
\hline Formulation & \multicolumn{5}{c}{ Peak Size Population } \\
\cline { 2 - 7 } & \multicolumn{2}{c}{ Peak 1 } & \multicolumn{2}{c}{ Peak 2 } & \multicolumn{2}{c}{ Peak 3 } \\
\cline { 2 - 7 } & Size $(\mathrm{nm})$ & Pop. (\%) & Size (nm) & Pop. (\%) & Size (nm) & Pop. (\%) \\
\hline $1: 1$ & 1231 & 80.2 & 193.7 & 10.9 & 4846 & 6.6 \\
$1: 5$ & 703.4 & 94.0 & 4755 & 6 & & \\
$1: 5$ & 682.1 & 83 & 134.6 & 10.3 & 5031 & 5.1 \\
\hline
\end{tabular}

The DLS size by intensity of each of the METx:GCPQ (glycerol) formulations is shown in figure 4.13 and the population percentage of particles under each peak is shown in 4.9, with the z-average, PDI and volume mean shown in table 4.10, Each ratio of METx:GCPQ (glycerol) produced particles within the nanometer range. However, all of the formulations also had a large ratio of particles above the nanometer range seen in figure 4.13. The increase in ratio of GCPQ from (1:1) to (1:5) shifted the distribution to the left, towards the nanometer range, however both (1:5) and (1:10) 
Table 4.10: DLS Z-average and PDI of METx:GCPQ (glycerol) formulations, (GCPQ batch: 210115, METx concentration $1 \mathrm{mg} \mathrm{ml}^{-1}$ ).

\begin{tabular}{cccc}
\hline $\begin{array}{c}\text { METx:GCPQ } \\
\text { Formulation }\end{array}$ & $\begin{array}{c}\text { Z-average }(\mathrm{nm}) \\
( \pm \mathrm{SD})\end{array}$ & $\begin{array}{c}\text { PDI } \\
( \pm \mathrm{SD})\end{array}$ & $\begin{array}{c}\text { Volume mean }(\mathrm{nm}) \\
( \pm \mathrm{SD})\end{array}$ \\
\hline $1: 1$ & $793.6(137)$ & $0.58(0.11)$ & $1236(449)$ \\
$1: 5$ & $394.6(68.9)$ & $0.53(0.13)$ & $887.9(3633)$ \\
$1: 10$ & $507.8(170)$ & $0.63(0.04)$ & $759.5(245)$ \\
\hline
\end{tabular}

formulations still showed some particles above the nanometer range. All three formulations showed a second smaller peak in the micron range, this did not change with an increase of GCPQ, this could be the aggregation of micelles. All the METx:GPCPQ (glycerol) formulations has similar PDI values between 0.58- 0.63.

\section{Role of Glycerol}

To help understand the role of glycerol in the METx:GCPQ (glycerol) formulations, METx:GCPQ (glycerol) formulations were prepared with either one or two of the components missing. The components of the formulation were GCPQ, AcOH and glycerol.

Table 4.11: Results of role of glycerol study.

\begin{tabular}{cc}
\hline Combination & Result \\
\hline METx + AcOH + Glycerol & Clear \\
METx + GCPQ + Glycerol & White suspension \\
METx + GCPQ + AcOH & Hazy suspension \\
METx + GPCQ & White suspension \\
METx + AcOH & Hazy suspension \\
METx + Glycerol & Suspension with precipitates \\
METx + AcOH redissolved after freeze dry & Hazy Suspension \\
\hline
\end{tabular}

Out of all the different combinations only METx:AcOH:glycerol produced a clear dispersion (shown in figure 4.14). METx:AcOH did not result in a clear dispersion which meant that $\mathrm{AcOH}$ was not able to disperse METx on its own. All the other combinations did not result in a clear dispersions. This suggested that glycerol was vital due to its ability to help disperse METx in $\mathrm{AcOH}$. This suggests that glycerol 
in this formulation is acting as co-solvent assisting in the solvation of a hydrophobic solute [366]. To confirm if $\mathrm{AcOH}$ was creating a acetate salt of METx, AcOH was removed by freeze drying and reconstituted in water. This produced a hazy suspension. This suggests that $\mathrm{AcOH}$ and glycerol work in a co-solvent system rather than the formation of an acetate salt of METx. 


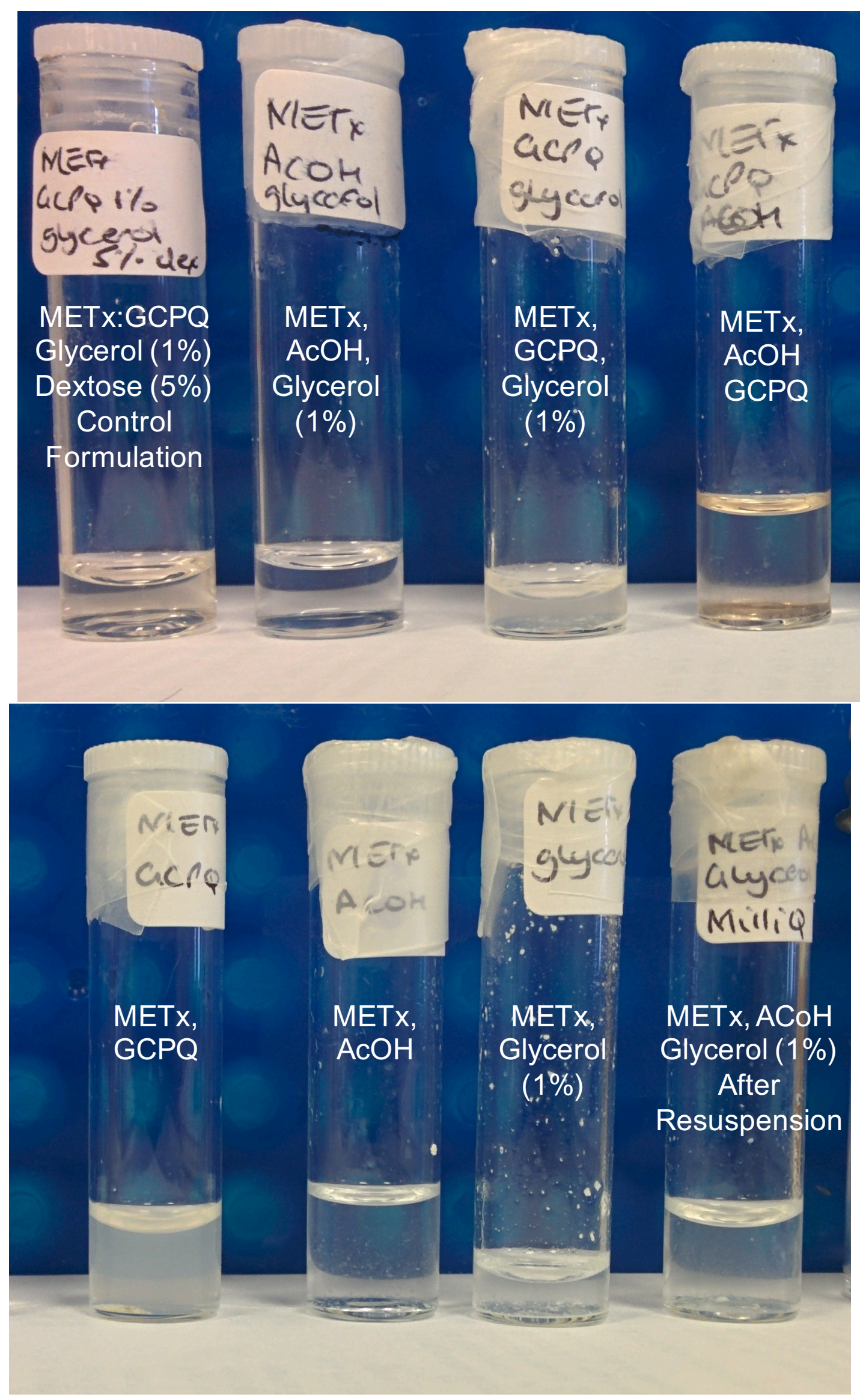

Figure 4.14: METx:GCPQ (glycerol) formulations prepared with either GCPQ, AcOH or glycerol omitted. 


\subsubsection{Stability Studies}

\subsubsection{Plasma Stability}

Plasma stability studies were performed in plasma diluted to $30 \%$ with saline $(0.9 \%)$. Plasma was diluted to help provide a slower break down such that it could be studied [183], this would allow for formulations to more deeply studied for their ability to protect METx. For METx:GCPQ (glycerol) formulations stability studies were also performed in $10 \%$ plasma as to provide a slower breakdown to generate a more clear ranking.

\subsection{METx:GCPQ Formulations}

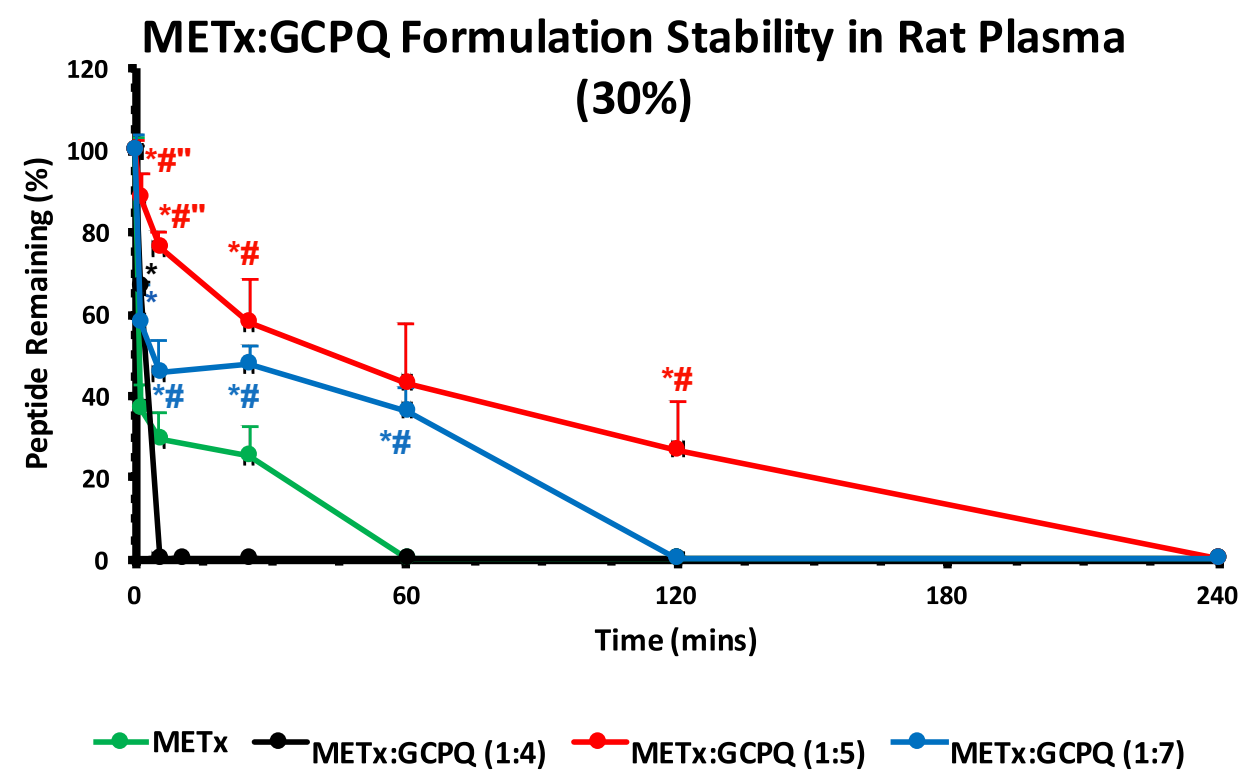

Figure 4.15: Stability of METx:GCPQ formulations (1:4), (1:5) and (1:7) in rat plasma (30\%), (final METx concentration 100 $\mathrm{mcg} \mathrm{ml}^{-1}$, GCPQ:051212). *= significant difference vs METx, \#= significant difference vs METx:GCPQ (1:4), "=significant difference vs METx:GCPQ (1:7); $<<0.05$.

All the METx:GCPQ formulations degraded over the 4 hour period, each reaching complete degradation over the four period. The ratio METx:GCPQ (1:5) was found to be the most stable, with the presence of METx for the longest period of time. METx:GCPQ (1:5) was found to be significantly different to METx at all time 
points except 4hr compared to METx alone. METx:GCPQ (1:5) was also significantly better than METx:GCPQ (1:7) at 1 and 5 min. METx:GCPQ (1:7) was the second most stable formulation. However, METx:GCPQ (1:4) degraded faster than METx alone, this could be attributed to the fact that this lower amount of GCPQ may not have encapsulated enough METx, but enhanced the solubility of METx which meant the degradation of METx:GCPQ (1:4) was quicker than METx alone.

\subsection{METx:GCPQ (Glycerol) Formulations}

\section{Plasma $(30 \%)$}

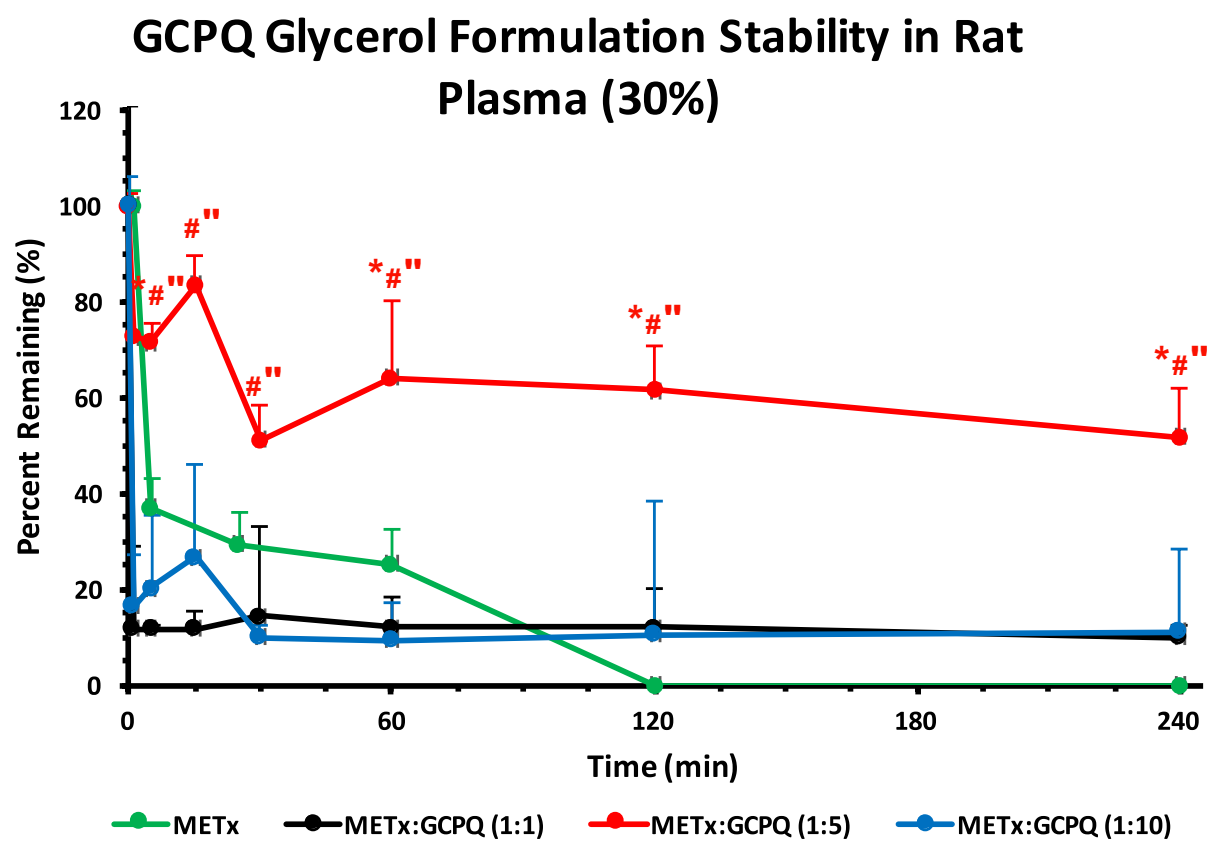

Figure 4.16: Stability of METx:GCPQ (glycerol) formulations (1:1), (1:5) and (1:10) in rat plasma (30\%), (final concentration of METx 100 $\mathrm{mcg} \mathrm{ml}^{-1}$, GCPQ:150615). *= significant difference vs METx,\#= significant difference vs METx:GCPQ (1:1), "=significant difference vs METx:GCPQ (1:10); $<<0.05$.

All the METx:GCPQ (glycerol) formulations degraded over the 4 hour period, none of the formulations reached complete degradation, with METx still being seen 
after 4 hours of degradation. The ratio METx:GCPQ (1:5) was found to be the most stable, with the largest presence of METx at all the time points over 4 hours. METx:GCPQ (1:5) was found to have significantly more METx at all the time points compared to METx alone. METx:GCPQ (glycerol) (1:5) was also significantly better than METx:GCPQ (glycerol) (1:1) and (1:10) at all the times over $4 \mathrm{hr}$. It was not possible to tell which was the second most stable as both METx:GCPQ (glycerol) (1:1) and (1:10) reached a similar percent of breakdown from 30 mins to 4 hours. To further help this ranking the area under curve was calculated of each formulation curve. The area under the curve was calculated to 2817 and 2819 for METx:GCPQ (glycerol) 1:1 and 1:5 respectively. As the difference in area is very small to help determine the second most stable glycerol formulation, all the formulations stability was also determined in plasma (10\%).

\section{Plasma (10\%)}

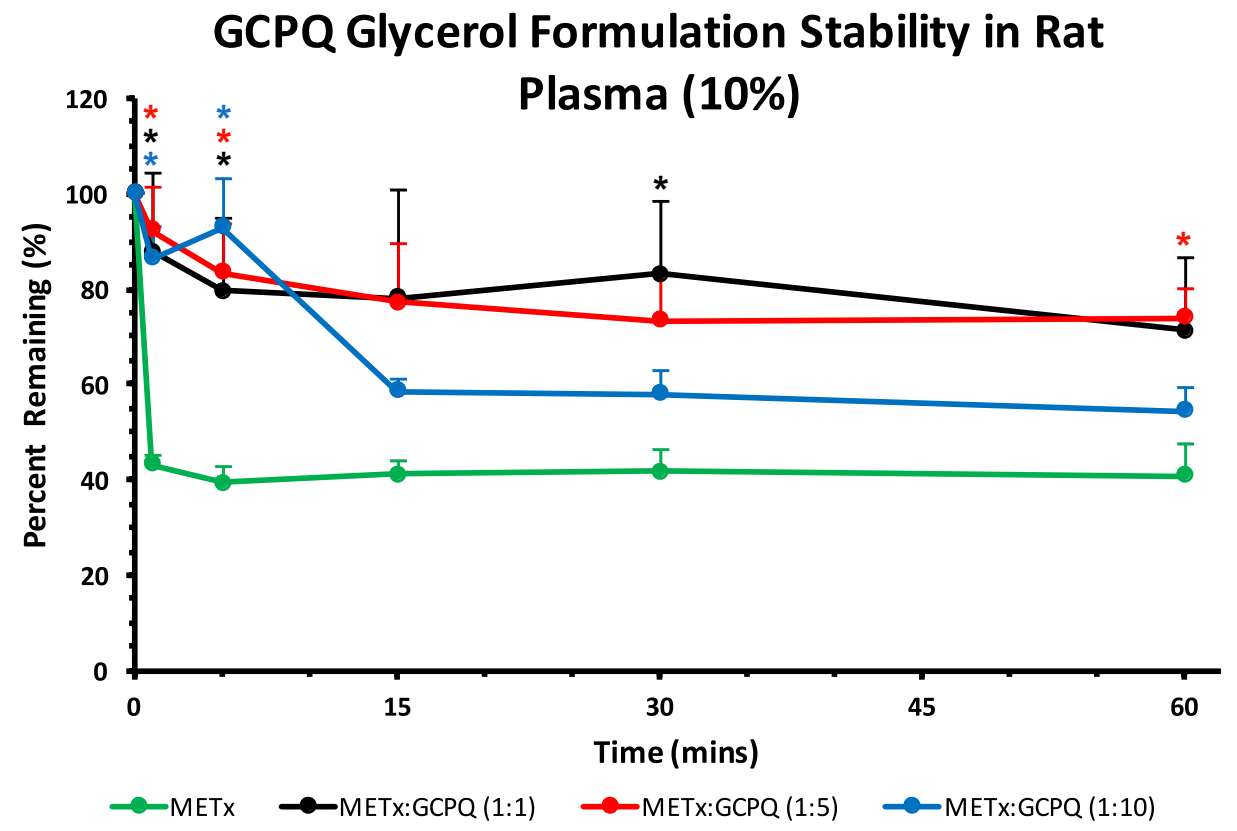

Figure 4.17: Stability of METx:GCPQ (glycerol) formulations (1:1), (1:5) and (1:10) in rat plasma (10\%) (final concentration of METx 100 $\mathrm{mcg} \mathrm{ml}^{-1}$, GCPQ:150615). *= significant difference vs METx,\#= significant difference vs METx:GCPQ (1:1), "=significant difference vs METx:GCPQ (1:10); $\mathrm{p}<0.05$. 
METx:GCPQ (glycerol) formulation stability was tested in plasma (10\%) to help determine a ranking for which formulations were the most stable in plasma. METx and all the formulations degraded over the 4 hour period, however none reached complete degradation. The ratio of METx:GCPQ (glycerol) (1:1) and (1:5) was found to be the most stable, however neither was significantly more stable than the other. METx:GCPQ glycerol (1:10) was more stable than METx alone, with significant difference at time points 1 and 5 minutes. METx (1:1) and METx (1:5) were not significantly different to each other at any time points. The stability study in plasma (10\%) did not help to provide a better rank as no formulation was able to provide a statistically better protection in plasma. As METx:GCPQ (glycerol) 1:1 and 1:5 produced similar stability the area under the curve was calculated for each formulation. The area under the curves were 4741 and 4588 for METx:GCPQ (glycerol) (1:1) and (1:5) respectively. Combining the area under the curve and the number of points that METx:GCPQ (1:1) and (1:5) are significantly different to METx, it can be suggested that METx:GCPQ (1:1) is the more stable formulation in plasma (10\%), it has the higher area under the curve. However both of these have the same number of significantly different time points. 


\subsection{Gastric Stability}

\section{METx:GCPQ Formulation Stability in SGF}

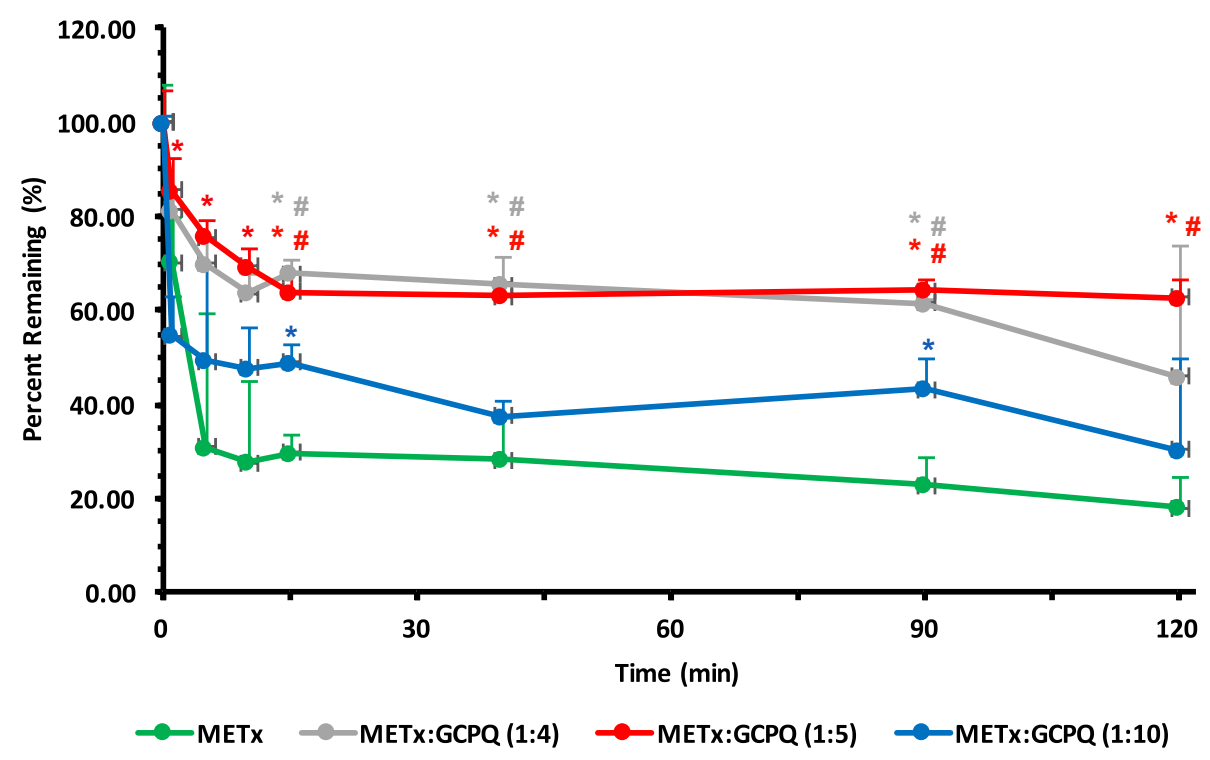

Figure 4.18: Stability of METx:GCPQ formulations (1:4), (1:5) and (1:10) in SGF (final concentration of METx $25 \mathrm{mcg} \mathrm{ml}^{-1}$, GCPQ:051212).*= significant difference vs METx,\#= significant difference vs METx:GCPQ (1:10); $<<0.05$.

All the peptide formulations degraded over the 2 hour time period. A ratio of METx:GCPQ (1:5) was found to be the least prone to degradation. GCPQ improved the stability of METx over the 2 hours. A greater amount of METx was recovered from METx:GCPQ 1:10 (30\%), METx:GCPQ 1:5 (62\%) and METx:GCPQ 1:4 (45\%) compared to METx alone (18.2\%) after 2 hours. The stability difference between MET:GCPQ (1:5) was significant compared to METx and METx:GCPQ (1:10) at times points 15, 40, 90 and 120 minutes. METx:GCPQ (1:4) was significantly different to METx:GCPQ (1:10) at 15, 40 and 90 minutes. The formulation METx:GCPQ 1:10 protected METx better than METx alone over 2 hours and was significantly different at 15 and 90 minutes. METx:GCPQ (1:4) and (1:5) formulations have a similarly best stability in SGF. 


\subsection{Intestinal Stability}

\section{METx:GCPQ Formulation Stability in RIW}

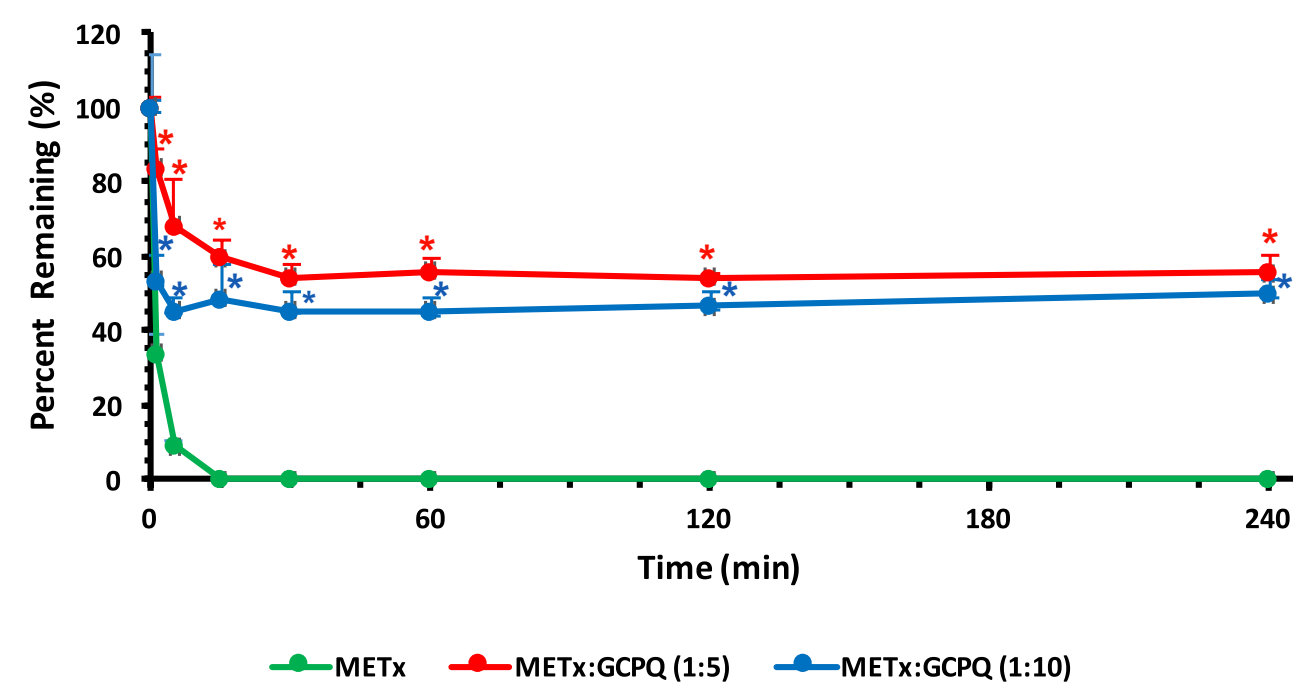

Figure 4.19: Stability of METx:GCPQ formulations (1:5) and (1:10) in RIW (final concentration of METx $25 \mathrm{mcg} \mathrm{ml}^{-1}$ GCPQ:051212), *= significant difference vs METx, \#= significant difference vs METx:GCPQ (1:100); $<<0.05$

All the peptide formulations degraded over the 4 hour time span. The presence of GCPQ improved the stability of METx over the 4 hours. METx in the absence of GCPQ was degraded to below detection within 5 minutes. A greater amount of METx was recovered from METx:GCPQ 1:10 (49.86\%) and METx:GCPQ 1:5 (55.7\%) compared to unencapsulated METx which was undetectable after 5 minutes. The difference between those formulations containing GCPQ was significantly better at all the time points 1 and 5 minutes unencapsulated METx. The two METx:GCPQ formulations were not significantly different from each other over the 4 hours except at the 1 minute time point. 


\subsection{Shelf Life Study}

\section{Stability Study: Peptide Content}

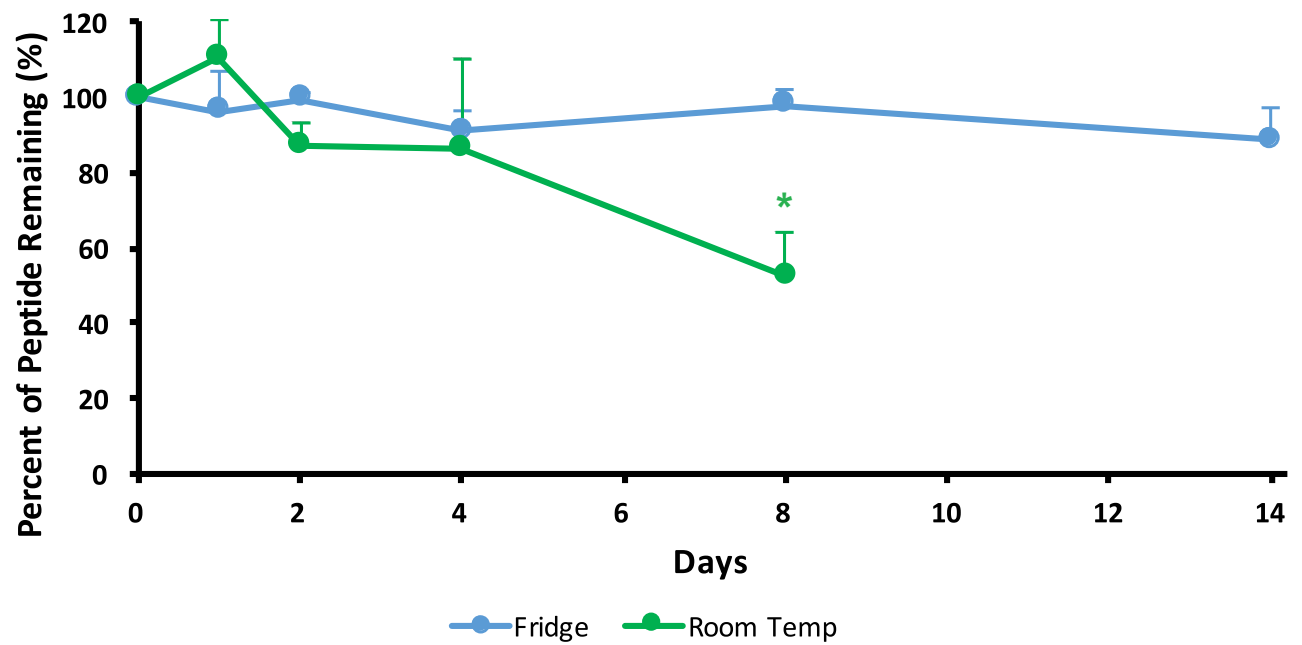

Figure 4.20: Peptide content of METx:GCPQ (glycerol) (1:5) stored at room temperature and at $4^{\circ} \mathrm{C}(\mathrm{GCPQ}: 050815), *=$ significant difference vs day $0 ; \mathrm{p}<0.05$.

\section{Stability Study: Formulation Size}

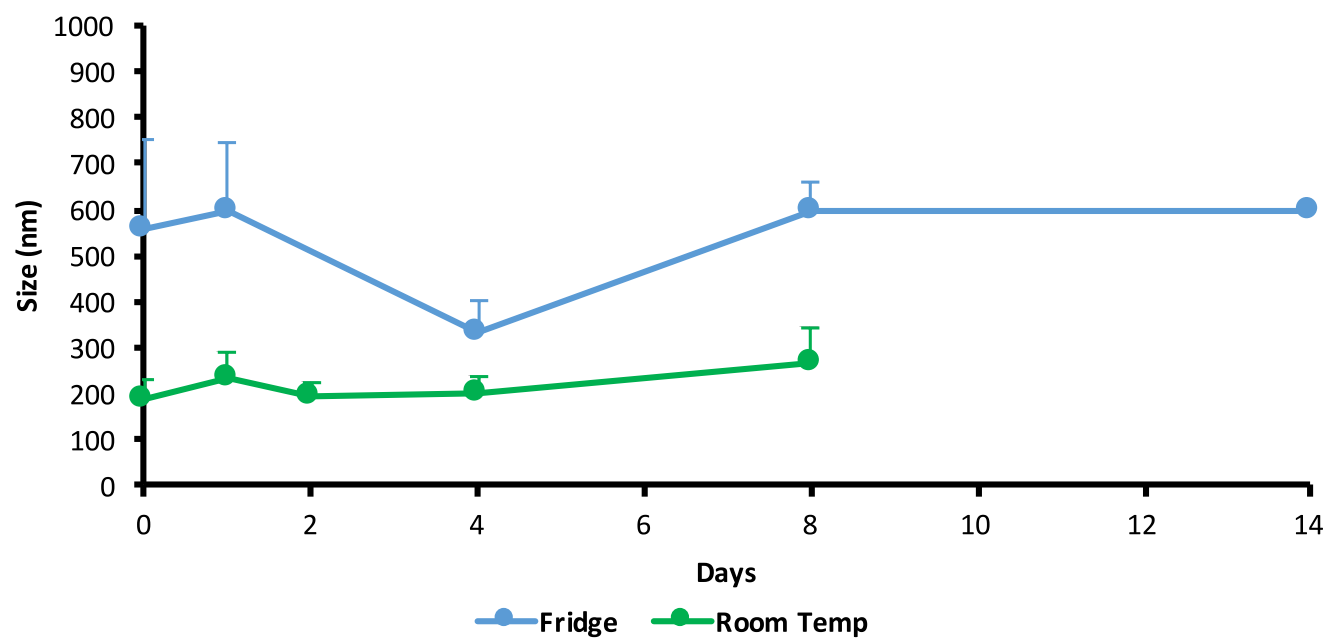

Figure 4.21: Nanoparticle size of METx:GCPQ (glycerol) (1:5) stored at room temperature and at $4^{\circ} \mathrm{C},(\mathrm{GCPQ}: 050815)$.

METx:GCPQ (glycerol) (1:5) was studied for its stability properties, to understand the stability of the freeze dried product. Individual aliquots of formulation were either 
stored at room temperature or at $4{ }^{\circ} \mathrm{C}$ (fridge). Upon reconstitution peptide content by HPLC and size by DLS was determined.

METx started to degrade within 2 days of being stored at room temperature, however the formulation stored in the fridge resulted in a similar peptide content over the two week period. The degradation of METx stored at room temperature was significant at day 8. The nanoparticle size of the formulation stored in both conditions did not change over the one week at room temperature and two weeks in at $4{ }^{\circ} \mathrm{C}$. The aliquots stored in the fridge at day 4 showed a drop in particle size, this drop was not significantly different to day 0 . 


\subsection{Discussion}

Chitosan, its derivative and modifications have been studied for more than 20 years to aid drug delivery resulting in more efficient drug delivery systems [338]. Chitosan has been modified by a number of methods in order to improve its drug delivery properties [367].

One of the greatest limitations of chitosan is that it is only soluble in acidic aqueous solutions, below pH 6.5 [337], meaning it will not be soluble everywhere within the body. GC is a chitosan derivative conjugated with ethylene glycol at the $\mathrm{C}_{6}$ position. The hydrophilic ethylene glycol substitution helps to increase the solubility at neutral and acidic pHs [344].

Modifications of GC help to produce an amphiphile [182]. The conjugation of hydrophobic moieties to GC help to create an amphiphile, which can form micelles at concentrations above the CMC. Common hydrophobic moieties include stearoyl, palmitoyl and octanoyl groups for chitosan based micelles [368, 369]. Within GCPQ palmitoyl $\left(\mathrm{C}_{16}\right)$ have been used as the hydrophobic moieties. The level of palmitoylation achieved was 21 (2.74) \% mol. A second modification was used to increase the polarity of the hydrophilic head group. Trimethylation of the PGC helps to aid its water solubility, as PGC is insoluble in water whereas GCPQ is soluble in water, an average quaternisation of $11(1.74) \% \mathrm{~mol}$ was achieved. The average molecular weight was $10.10(4.25) \mathrm{kDa}$ with a PDI of $1.14(0.10)$. The PDI is very low, this is probably due to the multiple dialysis steps which help to remove the smaller chains, that may have been degraded too much. A value of $0.89-1.15$ is regarded as good polymer homogeneity by the USP [370].

Comparing the two different methods used to synthesise GCPQ, the first method required a period of 30 days, where as the second method meant a larger amount of GCPQ could be synthesised in a shorter period of time, around 10 days [364]. The greatest change took place in the palmitoylation reaction, where the solvent was changed from EtOH to DMSO. The overall quaternisation reaction remains the same as developed by Domard et al. [371]. 
In order for a therapeutic agent to have an effect it is required to reach the site of action intact, for this it needs to be protected while being delivered and whilst travelling around in the systemic circulation. Two methods were used to make a formulation, one was probe sonicating METx and GCPQ and the other used glycerol as a co-solvent to create a METx:GCPQ solution.

To understand the role of glycerol in the METx:GCPQ (glycerol) formulation a study where each of the individual component's effect could be further understood. Formulations were made where one or two of the components were omitted. Figure 4.14 and table 4.11 show a picture and the appearance of each of these formulations. METx:AcOH:glycerol was the only formulation to produce a clear dispersion. As METx:AcOH was not a clear dispersion this suggests that glycerol is the key component to produce a clear dispersion, this suggests that glycerol acts a co-solvent within the medium. Glycerol is used within the pharmaceutical industry for a variety of formulations; oral, ophthalmic, topical, and parenteral preparations. It can be used as a preservative, emollient, gel vehicle, plasticiser, solvent and sweetening agent [372]. Co-solvents work by reducing the interfacial tension between the aqueous solution and hydrophobic solute [373]. Glycerol has been used a co-solvent in injectable formulations of dihydroergotamine and idarubicin, oral formulations of nifedipine, lopinavir and ritonavir (Kalerta) and etopside [374].

TEM and DLS nanoparticle size were used to help characterise each formulation. TEM is a technique that allows for the morphology of the formulation to be examined. Any peptide that may not be encapsulated can also be seen. TEM pictures of the METx:GCPQ formulations are shown in figure 4.6 to 4.8 and the TEM pictures of MET:GCPQ (glycerol) formulations are show in figure 4.10 to 4.12. In the TEM of both formulation techniques, it can be seen that as the ratio of GCPQ increases the amount of fibrous structures starts to decrease. METx due to its hydrophobicity is presumed to be encapsulated inside of the GCPQ core. This can be suggested by to the absence of METx fibrous structures in the TEM images of METx:GCPQ (1:10) and the presence of less fibrous structures in the METx:GCPQ (1:5) compared to METx:GCPQ (1:4). The TEM images of METx:GCPQ (glycerol) do not show a similar trend with the presence of some fibrous material throughout all of the ratios of 


\section{METx:GCPQ.}

The nanoparticle size distribution was measured by DLS. DLS can help to ascertain the overall range of sizes of the nanoparticle population and the PDI of the formulations. The size is a very important factor in any formulation; the size helps to determine which part of the body the formulation will travel to if the particle is absorbed [215, 360]. The size of the formulation was measured three times with a 15 second space between each measurement. This allows for any sedimentation or aggregation phenomenon to be taken into account. The METx:GCPQ and METx:GCPQ (glycerol) formulation's PDI did not change much with an increase in GCPQ ratio, a change of 0.07 and 0.05 . from the lowest to highest ratio respectively.

Figure 4.9, table 4.7 and 4.8 show the DLS population and values of METx:GCPQ formulations. The z-average size and PDI does not change largely through the formulations, however the size by volume measurement decreases. When comparing the peak size population, more than $95 \%$ of the population of particles are found in the nanometer range within the main peak, with a small in the micron size range.

The DLS data of METx:GCPQ (glycerol) formulations is shown in figure 4.13, table 4.9 and 4.10 . The z-average does not show a trend and the PDI value remain similar at $0.53-0.63$. The size volume decreases at the ratio of GCPQ increases in the METx:GCPQ (glycerol) formulations. When comparing the peak size populations, METx:GCPQ (1:1) glycerol and METx:GCP (1:10) glycerol formulations have two populations within the nanometer range where as METx:GCPQ (1:5) glycerol formulation has one main population. All of the METx:GCPQ (glycerol) have the majority of their particle population, more than $90 \%$, within the nanometer range, with a small percentage in the micron range.

The data from TEM and DLS do not provide the same answers, as TEM suggests the nanoparticles have a smaller size than those shown in DLS. The TEM also suggests that the nanoparticles would be less than 100nm and all have a similar size, whereas the DLS suggest that particles formed have a wide distribution from 100 to $1000 \mathrm{~nm}$. The results for DLS and TEM are similar to others that have reported lowering size values from TEM compared to those of DLS [375, 376]. Previously GCPQ has been used to 
deliver TPLENK, a hydrophobic peptide, formed similar sized micelles 200-500nm in size [139].

Both TEM and DLS use different techniques to measure the size; the data from both techniques has to be combined in order to form conclusions. The increased size of the data from the DLS could be due to aggregation and being present with a medium. DLS uses the Rayleigh theory to calculate hydrodynamic diameter, this assumes that the particles are completely spherical. This may not be entirely true. The volume size mean is calculated with 4 assumptions; all particles are spherical, all particles are homogenous, optical properties of the particles are known and there is no error in intensity distribution [363]. However, all of the METx:GCPQ and METx:GCPQ (glycerol) formulations particles are not homogenous as they have more than one population or a wide distribution, which means they infringe one of the assumptions, which means the volume mean values may be incorrect. TEM involves a process of applying a dye and drying which could affect the way the nanoparticles behave. Domingos et al. have studied DLS, TEM, atomic force microscopy (AFM), fluorescence correlation spectroscopy (FCS) and NTA to determine which technique is the most robust and reliable. They reported each technique has both its advantages and disadvantages andthat no single technique could be relied upon. In order to make conclusions all the techniques were required [377].

One of the biggest challenges in peptide drug development has been its stability in biological matrices. Peptides are susceptible to breakdown by proteases and peptidases due to peptide bonds [378]. A number of methods have been created one of which is encapsulating the peptide inside polymeric nanoparticles [379]. GCPQ is an amphiphilic polymer that forms micelles in water with a hydrophobic core. GCPQ has been found to help deliver a number of peptides orally that without encapsulation are rapidly degraded [139] or have unpredictable bioavailability [182].

Plasma stability of METx was tested by incubating METx in plasma diluted to 10 and $30 \%$ with saline $(0.9 \%)$. Plasma was diluted to slow down degradation so that it can be measured [139]. METx was found to reach complete degradation within 60 minutes in plasma (30\%). Although the plasma was diluted in this study, another study investigated the stability of OXT in undiluted plasma of normal, pregnant and 
lactating rats for 20 and 40 minutes. The results found OXT was stable in the plasma of all the types of rats with close $90 \%$ recovery at both 20 and 40 minutes [380]. Similar results have also been reported by Fabian et al where OXT only degraded by $20 \%$ over 24 hours, AVP however degraded much quicker with $50 \%$ remaining intact after 24 hours [381] . However, as most of the stability data were tested by showing OXT activity there has been suggestions that these values maybe incorrect as OXT has a half life of 3-6minutes in the body and degrades to metabolites that are more stable and have contribute to the measured levels of OXT activity [382].

Formulating METx with GCPQ in both the GCPQ and GPCQ (glycerol) formulations improved plasma stability. METx:GCPQ formulations with a weight ratio 1:5 and 1:7 showed higher plasma stability than METx, however when formulated with a weight ratio of 1:4, this formulation degraded faster than METx alone. Over the 4 hour study all the formulations completely degraded with no METx detectable at the 4 hours time point for all the formulations. METx:GCPQ (1:5) was found to be the most stable; METx:GCPQ (1:5) was found to be significantly more stable at all the time points.

METx:GCPQ (glycerol) formulations were tested for their plasma stability in 10 and 30\% (v/v) plasma. They were tested tested in two plasma concentrations to help create a ranking of which formulations were the most stable against plasma degradation. In plasma (30\%) METx:GCPQ (glycerol) (1:5) was found to the most stable with nearly $60 \%$ of the METx detectable at the end of 4 hours, where as the two other ratios, 1:1 and 1:10, degraded very quickly. In the first 30 minutes of the 4 hour study METx:GCPQ glycerol (1:1) and (1:10) formulations were degraded to $10 \%$ of METx detectable, after which there was very little degradation for the rest of the 4 hours as a similar 10\% was detected at the end of the 4 hours. METx:GCPQ (1:5) glycerol was found to be significantly more stable at all the time points compared to METx and the other METx:GCPQ (glycerol) formulations. The area under the curve of METx:GCPQ glycerol (1:1) and (1:10) was similar values 2817 and 2819 respectively, which meant the ratio $1: 1$ and 1:10 could not be ranked against each other.

As no clear ranking could be make between the superiority of METx:GCPQ (glycerol) (1:1) and (1:10) plasma (10\%) stability was performed. This further reduction 
in plasma would provide a slower degradation. METx was found not to fully degrade over the 4 hour period with $40 \%$ detectable at the $4 \mathrm{hr}$. All the degradation took place within the first few min after which there was very little degradation. All the METx:GCPQ (glycerol) formulations were more stable than METx with ratios of 1:5 and 1:1 showing the greatest stability. At the end of 4hr study METx:GCPQ (glycerol) (1:1) and (1:5) had over 70\% of METx still intact where as METx:GCPQ (glycerol) (1:10) had 55\%. None of the formulations degraded instantly like that seen in plasma (30\%) figure 4.16, they degraded steadily over the 4 hours. It is difficult to rank the glycerol formulations in terms of which protects METx the most as the ratio of 1:1 and 1:5 protect METx to a similar high degree in plasma (10\%) where as in the plasma (30\%) METx:GCPQ (glycerol) (1:1) did not protect METx at all. Comparing the area under the curve of the formulations METx:GCPQ glycerol (1:1) had a higher value, 4741, compared to MET:GCPQ glycerol (1:5). The overall ranking across both plasma (10 and 30\%) for METx:GCPQ (glycerol) formulations suggest a ratio of 1:5 is the best followed by $1: 1$ and then 1:10.

Previously studies of plasma stability of TPLENK:GCPQ formulations; showed a ratio of $1: 2.5$ to be completely stable in plasma. The results showed $100 \%$ of TPLENK detectable at the end of 8 hour study, however TPLENK alone showed some stability in plasma with over $60 \%$ detectable after 8 hours [139]. Another previous GCPQ peptide formulation of LENK:GCPQ showed similar improvements in stability. LENK:GCPQ formulations were found to be more stable than LENK alone in plasma, with LENK completely degrading after 2 hours where as LENK:GCPQ took more then $6 \mathrm{hr}$ to completely degrade [139]. Other studies using hydrophobically modified glycol chitosan for the delivery of unstable alkaloids found the use glycol chitosan micelles helped to retain stability for more than 10 hours [383]. However the GCPQ stability data shown from these experiments do not show the same level of stability against plasma.

Oral delivery of peptides has and will continue to receive a large amount of attention [7]. Oral drug administration is the most convenient and preferred medication administration route [68, 384] This improved convenience develops into an increased adherence with the dosing regimens prescribed leading to increased therapeutic efficacy [69]. To help create an oral formulation, METx:GCPQ formulations were studied 
for their stability in SGF and RIW.

Previous studies have found both OXT and AVP to be unstable in gastric juices [385]. Some have shown degradation within the first 5 minutes, with more than 50 percent lost. The results for METx were found to be similar with around $70 \%$ of the peptide being broken down in the first 5 minutes. In the first 15 minutes around $70 \%$ was broken down after which the rate of breakdown was reduced for the remainder of the 2 hours. Formulations with GCPQ helped to protect the peptide from breakdown. Increasing ratios of METx:GCPQ from 1:4 to 1:10 of GCPQ were tested, with a weight ratio of METx:GCPQ (1:5) found to be able to protect the peptide the most, indicated by the higher level of recovery after 2 hours. In the 2 hours of the study more than $60 \%$ of the peptide was protected when a ratio of METx:GCPQ (1:5) was used whereas, a ratio METx:GCPQ (1:4) has around 45\% and METx:GCPQ (1:10) had less than $30 \%$ of METx over 2 hours. The amount of peptide recovered after 2 hours was significantly better for the formulation METx:GCPQ (1:5) than METx at all the time points. The increased degradation in the formulation of METx:GCPQ (1:10) is thought to be due to an increased solubility factor, a greater amount of GCPQ has resulted in any unencapsulated METx becoming dissolved in the SGF thereby more prone to degradation by proteolytic enzymes.

The stability of METx in the presence of GCPQ in RIW have similar findings to other studies. METx has a similar structure to AVP. AVP has an intestinal stability of less than 5 minutes, however OXT was tested in the ileum where it completely degraded in less than 60 minutes [385]. Studies have also found that the main enzyme to cause AVP and OXT degradation is trypsin [386]. METx showed similar results, where within the first 5 minutes nearly all of the METx was broken down, to a point where it became undetectable. For formulations made using GCPQ, the stability was greatly increased. In the presence of GCPQ for both formulations METx:GCPQ (1:5 and 1:10) between 50-60\% of the peptide was still present after 4 hours. The amount of peptide protected in both GCPQ formulations was significantly better than unencapsulated METx at all time points. There was no significant difference between the formulations of METx:GCPQ 1:5 and 1:10 throughout the 4 hours.

To appreciate the stability of the freeze dried cake of METx:GCPQ (glycerol) the 
ratio 1:5 was stored at room temperature and in the fridge $\left(4^{\circ} \mathrm{C}\right)$. On each day the cake was resuspended and tested for METx content by HPLC and size of particles by DLS. The formulations stored at room temperature degraded within 2 days with the decreased peptide content becoming significantly different at day 8. For the formulation stored in the fridge, the METx content remained similar with no significant difference between the METx content on day 14 compared to day 0. The size by DLS for both formulations stored at room temperature and in the fridge did not change over the 8 and 14 days respectively.

Within this chapter it has been shown that METx may be formulated with GCPQ to improve its stability. GCPQ has been previously formulated to deliver LENK in an oral formulation. In RIW stability studies the LENK:GCPQ formulation protected LENK very well with close to $100 \%$ detectable after 6 hours, in comparison LENK degraded with around $20 \%$ was detectable at 6 hours. It must also be noted that within the first $2 \mathrm{hr}$ the LENK content decreased to $20 \%$, this means that the majority of the degradation took place in the first 2 hours [139]. Chitosan has been widely researched used for oral peptide delivery; one such method has been the use of chitosan-alginate nanoparticles. Chitosan based particles formulated by Zhang et al. were found to be completely stable in both gastric and intestinal medium [387]. The METx:GCPQ formulations were not able to provide a similar level of protection compared to those chitosan based formulations reported in the literature.

Thompson et al. used similar modifications of GCPQ on a polyallylamine polymer. The polymer was modified with palmitoyl and quartnerization head group and tested for it ability to deliver insulin. Stability was tested in both gastric and intestinal environments; it was found that insulin was unstable in both. Formulation of insulin with modified polyallylamine improved the stability significantly in both gastric and intestinal medium over a 4 hour period. The formulations provided greater protection in the intestinal mediums compared to the gastric medium, with 50-90\% and 25\% detectable in each medium respectively at the end of the study [388]. This helps to highlight impact of the polymer back bone in gastric stability. The METx:GCPQ stability data shows similar stability profiles whereby GCPQ is able to help improve stability, but not completely protect METx. 


\section{Chapter 5: In Vivo Studies}

\subsection{Introduction}

\subsubsection{Physiology of Urine Production}

When a large volume of water has been consumed by the body the blood plasma becomes diluted and the urine produced by the body is more dilute than plasma. If the water intake is small this results in concentrated blood plasma which means the urine produced is more concentrated [389]. After a nights sleep or after not consuming water for several hours the urine osmolality would be around $1200 \mathrm{mOsm} \mathrm{L}^{-1}$ nearly 4 times that of plasma [389].

The glomerulus filters around $130 \mathrm{ml}$ per $1.73 \mathrm{~m}^{2}$ per minute in young men and $120 \mathrm{ml}$ per $1.73 \mathrm{~m}^{2}$ per minute in young women [390]. However only a small percent of this volume is excreted daily as urine; the average young adult producing between 1.6 to $1.8 \mathrm{~L}$ of urine per day [391]. The concentration of urine and the reabsorption of water and salts to match the body's requirements is due to complex systems within the nephrons of the kidneys [392]. Figure 5.1] shows an overview of this concentration process within the nephrons of the kidney.

The majority of the fluid filtered by the glomerulus is reabsorbed in the proximal tubule. The proximal tubule is freely permeable to water due to the constant expression of aquaporin-1 (AQP1) channels. This results in constant water reabsorption, regardless of the state of the body [393]. The AQP1 is also expressed in the upper parts of the descending thin limbs of the loop of Henle [394]. AQP1 water channels 


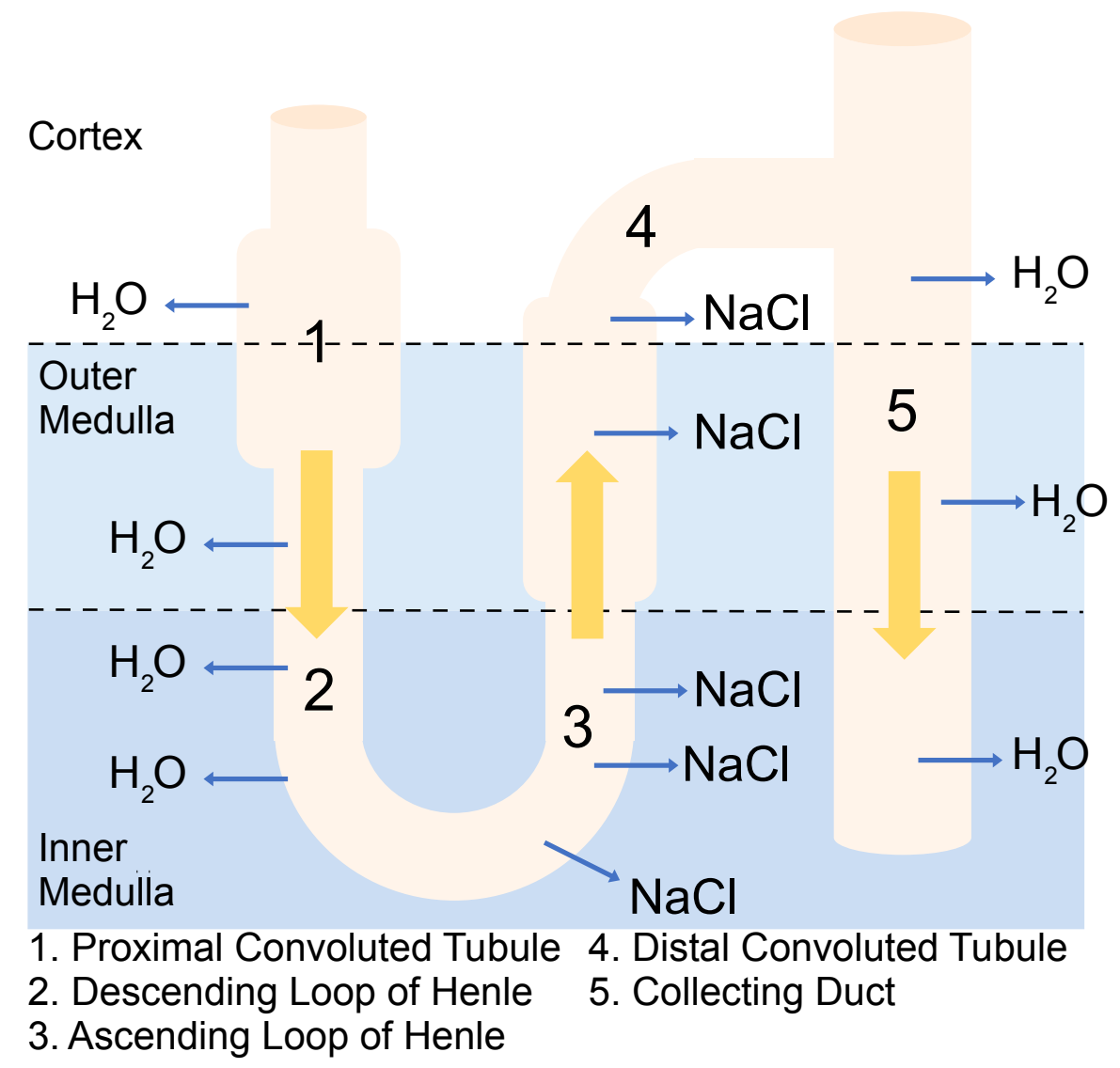

Figure 5.1: Concentrating of Urine in Nephrons of the kidney.

are responsible for most of the water reabsorption in the nephrons of the kidney [395], whereas the AQP2 water channels are able to adjust the volume of urine to the body's osmolaltiy requirements [396].

The AQP1 is found in the upper parts of the thin descending limb of the loop of Henle [397]. The AQP1 accounts for the majority of filtrate reabsoration as it is freely permeable to water owing [398]. Urine then enters the thin ascending limb. The thin ascending limb is impermeable to water but permeable to sodium and urea [399], as seen in section 2 and 3 of figure 5.1. The thin descending limb and thin asending limb uses countercurrent to concentrate urine [389]. Urine then enters the thick ascending limb which actively removes sodium chloride via co-transporters, NKCC2 on the apical membrane and N-K-ATPase transporters in basolateral membrane [400]. The urine is further concentrated in the cortex so that the urine's osmolality is less than that 
the of blood [389]. The urine finally enters the collecting ducts where the water content of urine is fine tuned. In the absence of AVP the collecting ducts are impermeable to water. The collecting ducts become highly permeable to water in the presence of AVP [401].

\subsubsection{Urine Disorders}

Many patients report polyuria and polydipsia together and share the same reasons for presentation [402]. Diabetes mellitus is the most common reason of polyuria with more than 1 in 20 in the UK suffering from diabetes mellitus [403]. Table 5.1] shows the most common cause of polyuria, where treatment involves tackling the underlying cause.

Table 5.1: Common causes of polyuria [402]

\begin{tabular}{cc}
\hline Common $(>1$ in 10) & $\begin{array}{c}\text { Diuretics/caffeine/alcohol } \\
\text { Diabetes mellitus } \\
\text { Lithium } \\
\text { Heart Failure }\end{array}$ \\
Infrequent $(1$ in 100$)$ & $\begin{array}{c}\text { Hypercalcaemia } \\
\text { Hyperthyroidism }\end{array}$ \\
Rare $(1$ in 1,000$)$ & $\begin{array}{c}\text { Chronic renal failure } \\
\text { Primary polydipsia } \\
\text { Hypokalaemia }\end{array}$ \\
Very rare $(<1$ in 10,000$)$ & Diabetes insipidus \\
\hline
\end{tabular}

Another urine disorder is nocturia, which is defined by The International Continence Society as waking at night to void, with each void preceded and followed by sleeps [404]. This is experienced by up to $61 \%$ of elderly women and up to $59 \%$ of elderly men reporting two or more voids per night. The prevalence of nocturia increases with age, with underlying causes being a mix of more than one factor. Factors can include; reduced bladder capacity, renal insufficiency, congestive heart failure, venous insufficiency, hypercalcemia to list a few [405].

Recently there has been an interest in treating nocturia polyuria with desmopressin. A review by Friedman and Weiss of clinical trials for the treatment of nocturia with 
desmopressin found that in 9 clinical trials with desmopressin patients reported significantly less nocturia then those on placebo. In the 9 clinical trials 7 received oral desmopressin and 2 received desmopressin sublingually. They found that each clinical trail supported the use of desmopressin in nocturia [406]. In another review by Ebell et al for the use of desmopressin for nocturia in adults, data from 10 clinical trials were reviewed and a modest improvement in nocturia was reported. Across the trials a decrease of $17 \%$ in voids per night was reported [407].

A similar urine disorder reported by many young children and parents is nocturnal enuresis (NE), i.e. bed-wetting. It is a condition where uncontrollable leakage of urine takes place during sleep in children over the age of 5 [408]. In some cases this can be the only presenting symptoms however other symptoms also reported include; daytime incontinence, urgency, decreased void frequency during the day and pain on going [409]. There are two types of NE; primary where children have never been able to achieve a period in which enuresis is not experienced or secondary enuresis where children are able to have at least a 6 month period without enuresis [410]. Secondary enuresis is most likely triggered by a stressful life event [411]. It is thought that $15 \%$ of children suffer from NE with $80 \%$ of NE primary enuresis [410], with the prevalence decreasing with age with around 3\% of 10-12 year olds reporting the condition [412]. The most common reasons for NE are increased urine production while sleeping, reduced bladder capacity, developmental delay and genetics [413].

Two lines of therapy are used for NE; alarms and desmopressin. The alarm is an electronic device that produces a loud sound when it senses an episode of incontinence. It is can be worn or placed in the bed. The other first line treatment can be the use of desmopressin. A Cochrane review into the use of desmopressin for NE found desmopressin was effective in reducing bed-wetting compared to placebo. However, once desmopressin treatment was stopped, NE resumed similar to that of placebo [414]. Another study compared 6 month use of no treatment, alarm, desmopressin and imipramine, a tricyclic antidepressant. They found that compared to no treatment, any form of treatment helped reduced NE over the 6 month period. Only $6 \%$ of untreated patients reported no NE at the end of 6 months compared to desmopressin $(68 \%)$, alarm (63\%) and impramine (36\%). However, once treatment was stopped 
from months 6 to 12 only the alarm group reported a persistent reduction in NE [415].

Our data ha shown the ability of METx to function as a $V_{2} R$ partial agonist. Formulating METx with GCPQ may provide a means to deliver METx. AVP agonists ability to reduce the over production and passing of urine is used conditions such as diabetes insipidus, polyuria, nocturia and bed-wetting. The experiments in this chapter tested the METx formulations for its ability to reduce the production of urine in rats. The efficacy of the formulation was determined by the volume of urine passed and the osmolality of the passed urine. 


\subsection{Materials Methods}

\subsubsection{Materials}

Table 5.2: Materials used for in vivo studies of METx.

\begin{tabular}{ll}
\hline Materials & Supplier \\
\hline Dextrose & Sigma-Aldrich \\
GCQP & Synthesised in chapter 4 \\
Glycerol & Fisher \\
METx & Severn Biotech \\
Sodium hydroxide & Sigma-Aldrich \\
\hline
\end{tabular}

\subsubsection{Methods}

\subsubsection{Animals}

All the experiments were performed under a Home Office License No. PPL 70/8224 (Animals (Scientific Procedures) Act 1986). Male Sprague Dawley rats (Harlan, Indianapolis, USA) were used when their weights were around 220-250 g, animals were weighed on the morning of the experiment.

The rats were housed five per cage in an air conditioned unit maintained at 20$22^{\circ} \mathrm{C}$ and 50-60\% humidity, and were allowed free access to standard rodent chow and water. Lighting was controlled on a twelve-hour cycle, lights on at 07:00 and off at 19:00. Animals were habituated for 7 days prior to experimentation and acclimatised to the procedure room for 1 hour prior to testing. Animals were also acclimatised to the metabolic cages for 20 minutes per day for 5 days before the day of experiment. 


\subsubsection{Administration}

\subsection{Intravenous Study}

Animals were randomly assigned into one of four groups; untreated, 10, 20 or $40 \mathrm{mg} \mathrm{kg}^{-1}$ of METx:GCPQ ( $\mathrm{n}=3$, GCPQ batch: 051212). The formulations were prepared fresh on the morning of the experiment as described 4.2.2.2.1 in and the $\mathrm{pH}$ was titrated to $\mathrm{pH} 7$ using $\mathrm{NaOH}$. The ratio of METx:GCPQ (1:5) was used to make these formulations.

Animals tails were heated using a warm cloth to stimulate dilation of the tail vein. The tail vein was cleaned using ethanol $(70 \%)$ and were given the required formulation $(0.5 \mathrm{ml})$ by IV injection via the tail vein. After administration each animal was placed in an individual metabolic cage (Techniplast, Italy) with access to water. Spontaneously voided urine was collected for 4 hours. Urine osmolality was determined by freezing point depression using a Type 5r osmometer (Loser, Germany).

\subsection{Oral Study}

The animals were fasted overnight (16 hours) with access to water. Animals were randomly assigned to two groups; vehicle (GCPQ, GCPQ batch:121114) or METx:GCPQ (n=5). Two separate studies were performed; 100 and $200 \mathrm{mg} \mathrm{kg}^{-1}$. A ratio of METx:GCPQ (1:4) was used, this was used as ratio of METx:GCPQ (1:5) was too viscous to be delivered by oral gavage.

The formulations were prepared fresh on the morning of the experiment as described in section 4.2.2.2.1 and were given the required formulation ( $1 \mathrm{ml})$ to rats by oral gavage (Torpac, New Jersey, USA) as per designated group. After administration each animal was placed in an individual metabolic cage (Techniplast, Italy) with access to water. Spontaneously voided urine was collected for 4 hours. Urine osmolality was determined by freezing point depression using a Type 5r osmometer (Loser, Germany). 


\subsection{Subcutaneous Study}

Two separate studies were performed using either the METx:GCPQ or METx:GCPQ (glycerol) formulation. Animals used for METx:GCPQ formulation were assigned to one of three groups at random; vehicle (GCPQ, GCPQ batch: 210115), 10 or 20mg $\mathrm{kg}^{-1}$ of METx:GCPQ $(\mathrm{n}=5)$. Animals used for the METx:GCPQ (glycerol) formulation study were assigned to one of four groups; vehicle (GCPQ, GCPQ batch:030915), 10, 20 or 40mg kg-1 of METx:GCPQ (glycerol) formulation $(\mathrm{n}=5)$.

The formulations were prepared fresh on the morning of the experiment as described in 4.2.2.2.1 and 4.2.2.2.2 for the METx:GCPQ and METx:GCPQ (glycerol) subcutaneous study respectively. The animals were injected with the required formulation $(1 \mathrm{ml})$ subcutaneously at the loose skin around the neck. After administration each animal was placed in an individual metabolic cage (Techniplast, Italy) with access to water. Spontaneously voided urine was collected for 4 hours. Urine osmolality was determined by freezing point depression using a Type 5r osmometer (Loser, Germany). 


\subsection{Results}

\subsubsection{Intravenous Study}

\subsubsection{Urine Volume}

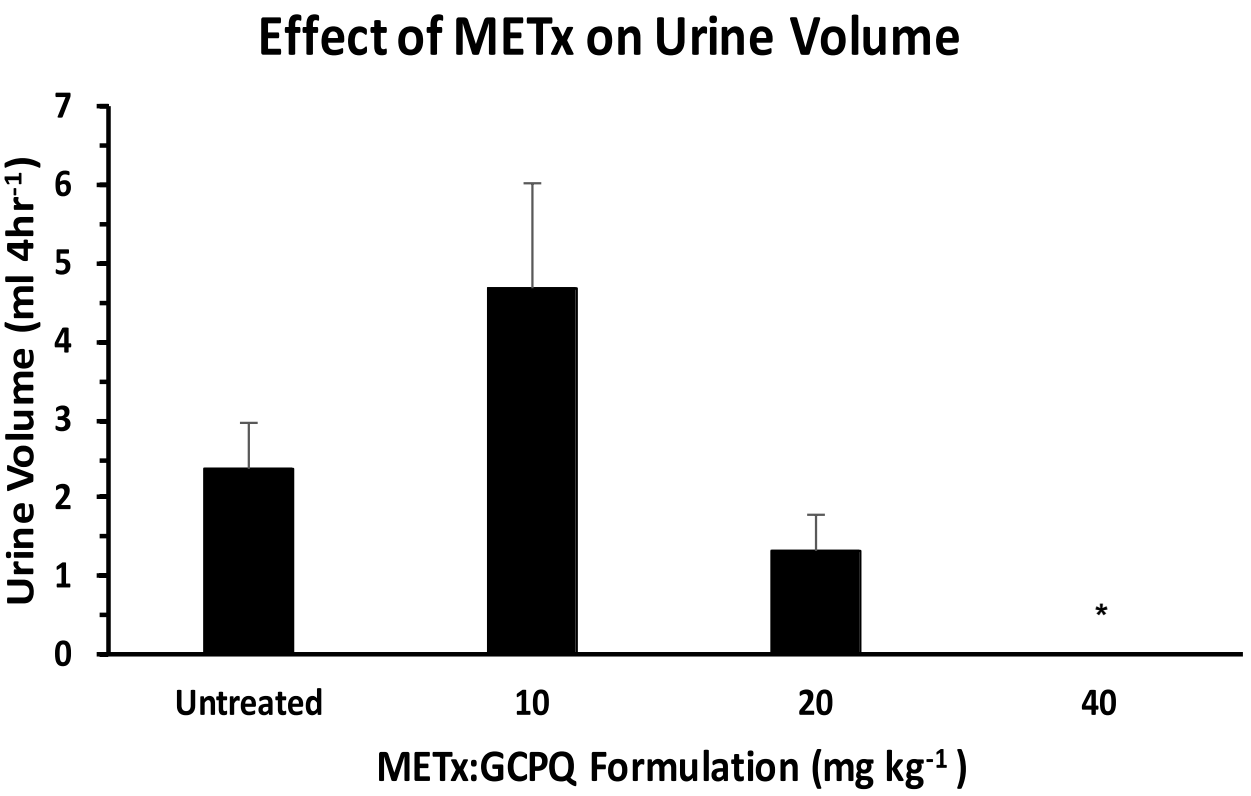

Figure 5.2: Effect of intravenous administration METx:GCPQ on urine volume of male SD rats over 4 hours $(\mathrm{n}=3)$, (GCPQ: 051212); *=significant difference to control $\mathrm{p}<0.05$.

Male SD rats $(n=3)$ were dosed intravenously via the tail vein with METx:GCPQ (1:5) at dose of 10,20 or $40 \mathrm{mg} \mathrm{kg}^{-1}$, after which they were placed individually into metabolic cages. Control animals were not dosed. The results for urine production are shown in figure 5.2. The spontaneously voided urine was collected over 4 hours. METx caused a dose responsive decrease in urine volume. The urine volume for untreated animals was $2.3 \mathrm{~mL}$. The rats dosed with METx $\left(40 \mathrm{mg} \mathrm{kg}^{-1}\right)$ did not produce any urine at all, with one animal having to be put down due to discomfort. The urine produced at METx (40mg kg ${ }^{-1}$ ) was statistically significantly different to the control. The two other doses 10 and $20 \mathrm{mg} \mathrm{kg}^{-1}$ urine volume were not significantly different to that of the control. 


\subsubsection{Urine Osmolality}

\section{Effect of METx on Urine Osmolality}

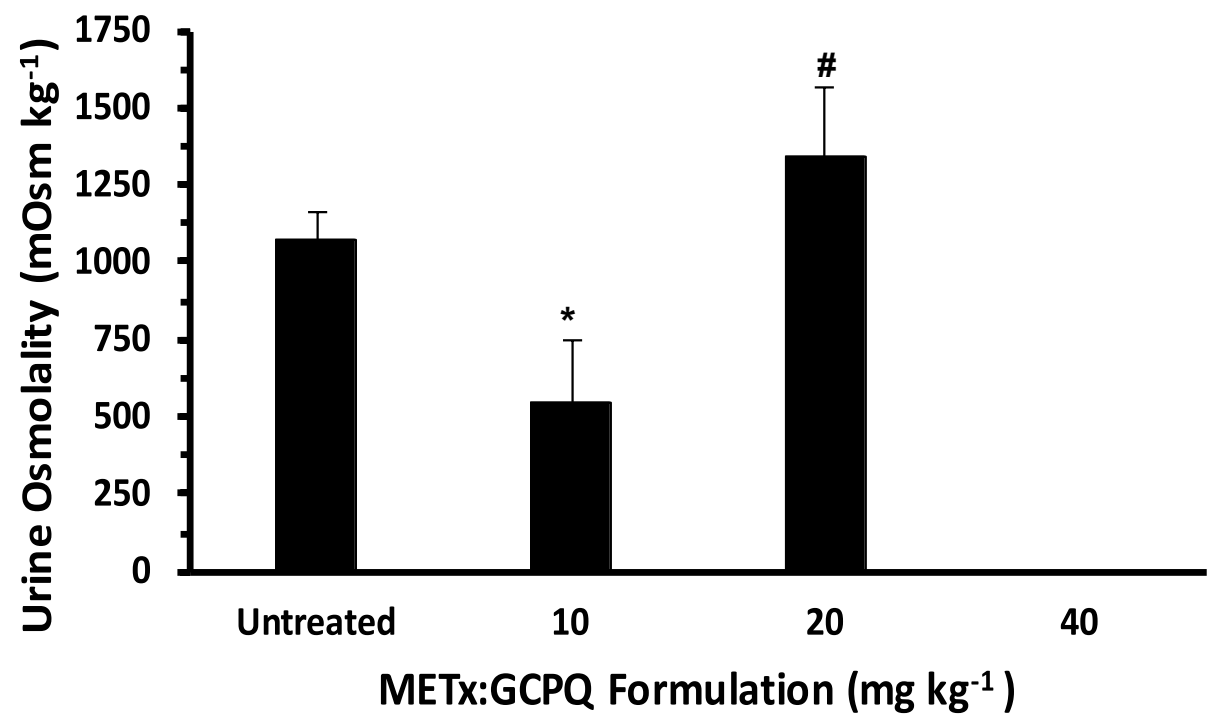

Figure 5.3: Effect of intravenous administration METx:GCPQ on urine osmolality of male SD rats over 4 hours $(n=3) ; *=$ significant difference to untreated, (GCPQ:051212), \#=significant difference to $10 \mathrm{mg} \mathrm{kg}^{-1}, \mathrm{p}<0.05$.

Urine produced by male SD rats $(n=3)$ dosed intravenously via the tail vein with METx:GCPQ (1:5) at doses 10, 20 or $40 \mathrm{mg} \mathrm{kg}^{-1}$ was collected over 4 hours. The urine's osmolality was measured by an osmometer, freezing point depression osmometer. The results are shown in figure 5.3. The urine osmolality of the control rats, produced urine which had an osmolality of approximately $1000( \pm 93) \mathrm{mOsm} \mathrm{kg}^{-1}$. The urine osmolality increased from as the dose was increased from 10 to $20 \mathrm{mg}$ $\mathrm{kg}^{-1}$. However as there was no urine produced at $40 \mathrm{mg} \mathrm{kg}^{-1}$ no osmolality could be measured. A trend would be expected that with a lower urine volume, a higher osmolality would be seen, as there would be a higher concentration of solute within a lower volume of fluid. It is unclear if that trend is being shown within this urine osmolality as there are only two values to compare against. However there is a significant difference between the urine osmolality of 10 and $20 \mathrm{mg} \mathrm{kg}^{-1}$. There was no significant difference between the control and $20 \mathrm{mg} \mathrm{kg}^{-1}$ group. 


\subsubsection{Oral Study}

\subsubsection{Urine Volume}

\section{Effect of METx:GCPQ on Urine Production: Oral Delivery}

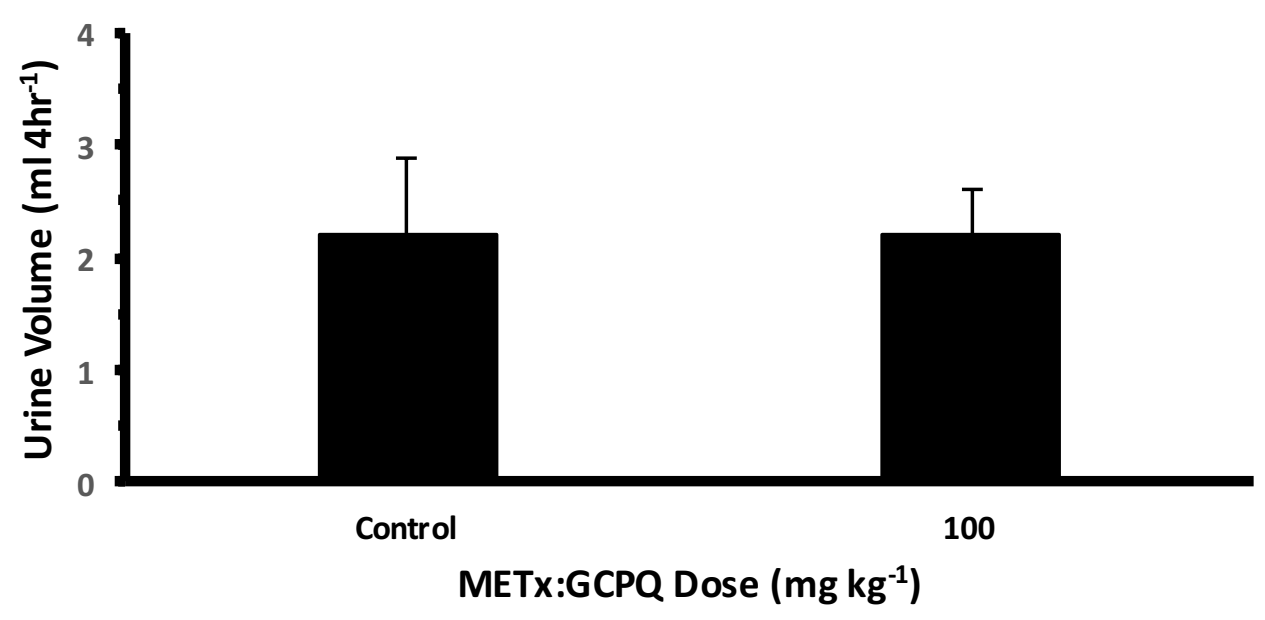

Figure 5.4: Effect of oral administration METx:GCPQ (1:4) $\left(100 \mathrm{mg} \mathrm{kg}^{-1}\right)$ on urine volume of male SD rats over 4 hours ( $n=5)$, (GCPQ:121114).

Male SD rats ( $n=5)$ were dosed either GCPQ (control) or METx:GCPQ (1:4) (100 $\mathrm{mg} \mathrm{kg}^{-1}$ ) via an oral gavage and placed individually inside metabolic cages. Spontaneously voided urine was collected for 4 hours. For the $200 \mathrm{mg} \mathrm{kg}^{-1}$ dose a ratio of METx:GCPQ (1:4) has to be used rather than METx:GCPQ (1:5) to reduce the viscosity of the formulation. This was chose as METx:GCPQ (1:4) was chosen as it a similar breakdown profile to METx:GCPQ (1:5) (see figure 4.18) and it was more doseable. The results are shown in figure 5.4. There was no difference in the urine volume produced by METx compared to that of the control.

As there was no urine production difference in rats dose METx:GCPQ $(1: 4,100 \mathrm{mg}$ $\mathrm{kg}^{-1}$ ) a double dose was tried to examine if any urine effects could be observed. Male SD rats $(\mathrm{n}=5)$ were dosed with either GCPQ (control) or METx:GCPQ (1:4, $200 \mathrm{mg}$ $\mathrm{kg}^{-1}$ ) via oral gavage and placed individually into metabolic cages. Spontaneously voided urine was collect for 4 hours. The results are shown in figure 5.5 There was no difference seen in the urine production between the control and METx animals. 


\section{Effect of METx:GCPQ on Urine Production: \\ Oral Study}

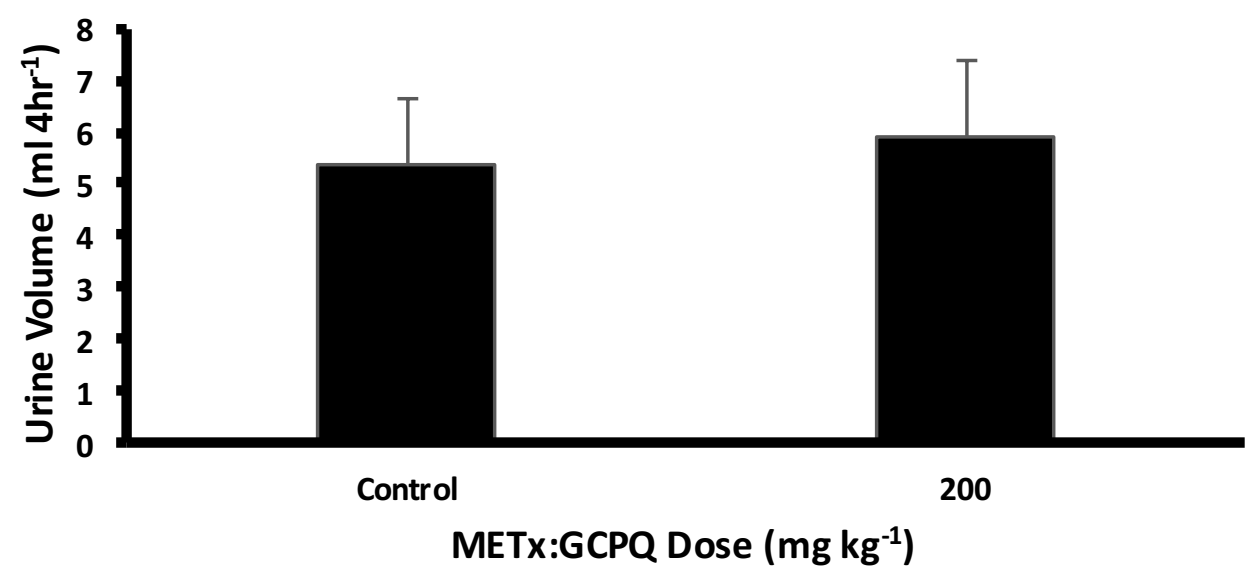

Figure 5.5: Effect of oral administration METx:GCPQ (1:4) $\left(200 \mathrm{mg} \mathrm{kg}^{-1}\right.$ on urine volume of male $\mathrm{SD}(\mathrm{n}=5)$ rats over 4 hours, (GCPQ:121114).

The results from the two oral studies at 100 and $200 \mathrm{mg} \mathrm{kg}^{-1}$, showed that METx:GCPQ formulation is not orally active at reducing the urine output. This can be due to a combination of reasons such as; the METx:GCPQ formulation is not stable enough within the GI tract, not able to pass into the systemic circulation, goes under significant first pass metabolism and/or the formulation is not stable enough within the plasma to reach the site of action. 


\subsubsection{Urine Osmolality}

\section{Effect of METx:GCPQ on Urine Osmolality: Oral Delivery}

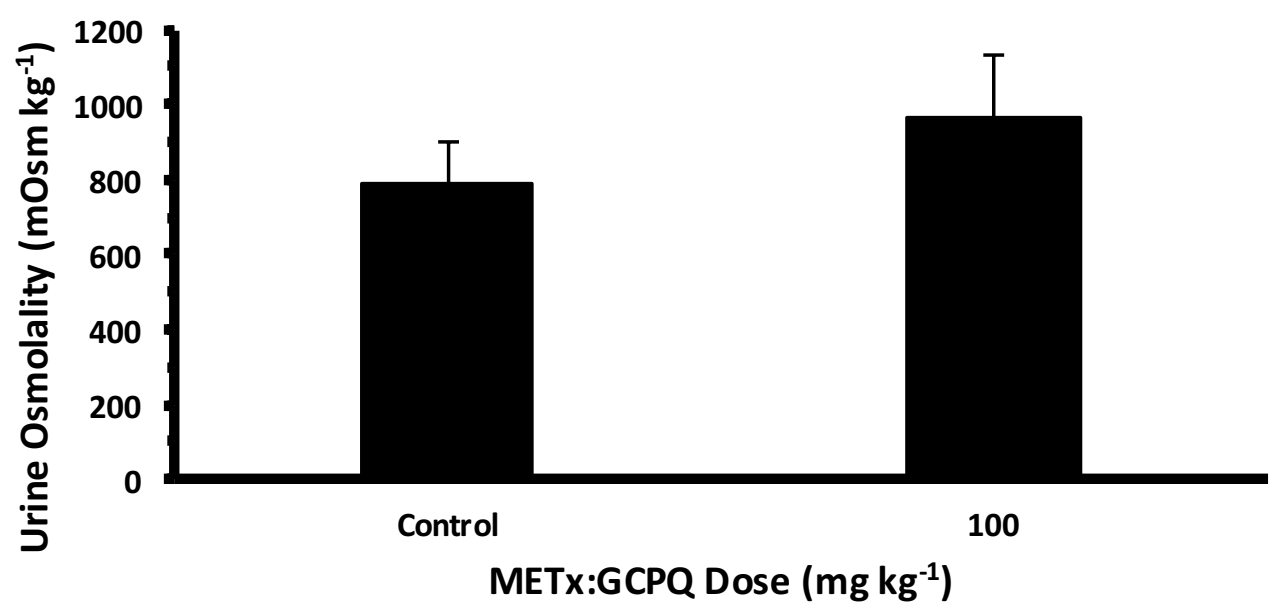

Figure 5.6: Effect of oral administration METx:GCPQ $\left(100 \mathrm{mg} \mathrm{kg}^{-1}\right)$ on urine osmolality of urine produced by male SD rats over 4 hours (n=5), (GCPQ:121114).

\section{Effect of METx:GCPQ on Urine Osmolality: Oral Study}

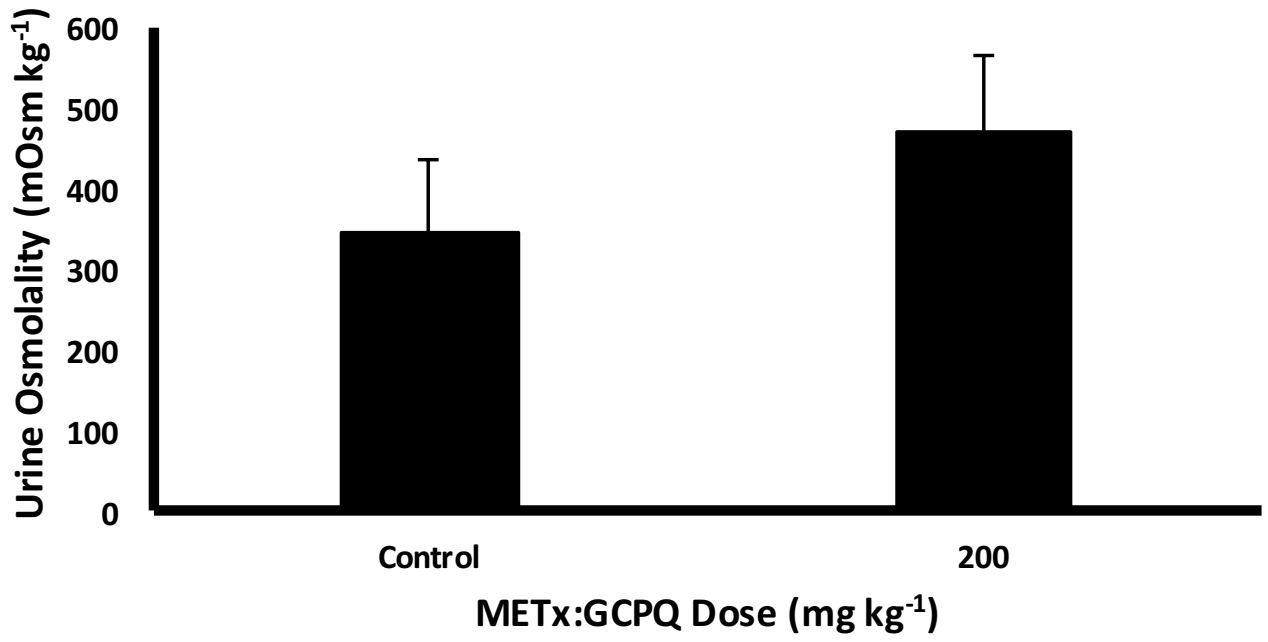

Figure 5.7: Effect of oral administration METx:GCPQ $\left(200 \mathrm{mg} \mathrm{kg}^{-1}\right)$ on urine osmolality of urine produced by male SD rats over 4 hours ( $\mathrm{n}=5)$, (GCPQ: 121114).

The urine osmolality of urine produced by the rats dosed either control or METx:GCPQ orally was measured using the freezing point method. The results for 100 and $200 \mathrm{mg}$ $\mathrm{kg}^{-1}$ are show in figure 5.6 and 5.7 respectively. The urine osmolality after dosing 
with METx at a dose of 100 and $200 \mathrm{mg} \mathrm{kg}^{-1}$ was similar to the control values for urine. This was expected as the oral formulations did not produce any difference in urine volume, which would suggest there was no difference in water reabsorption at the collecting duct. The lack of difference in water reabsorption would result in urine of similar concentration being produced by the control and animals dosed METx orally.

\subsubsection{Subcutaneous Study}

\subsubsection{Urine Volume}

\subsection{METx:GCPQ Formulation}

\section{Effect of METx:GCPQ on Urine Production: Subcutaneous Delivery}

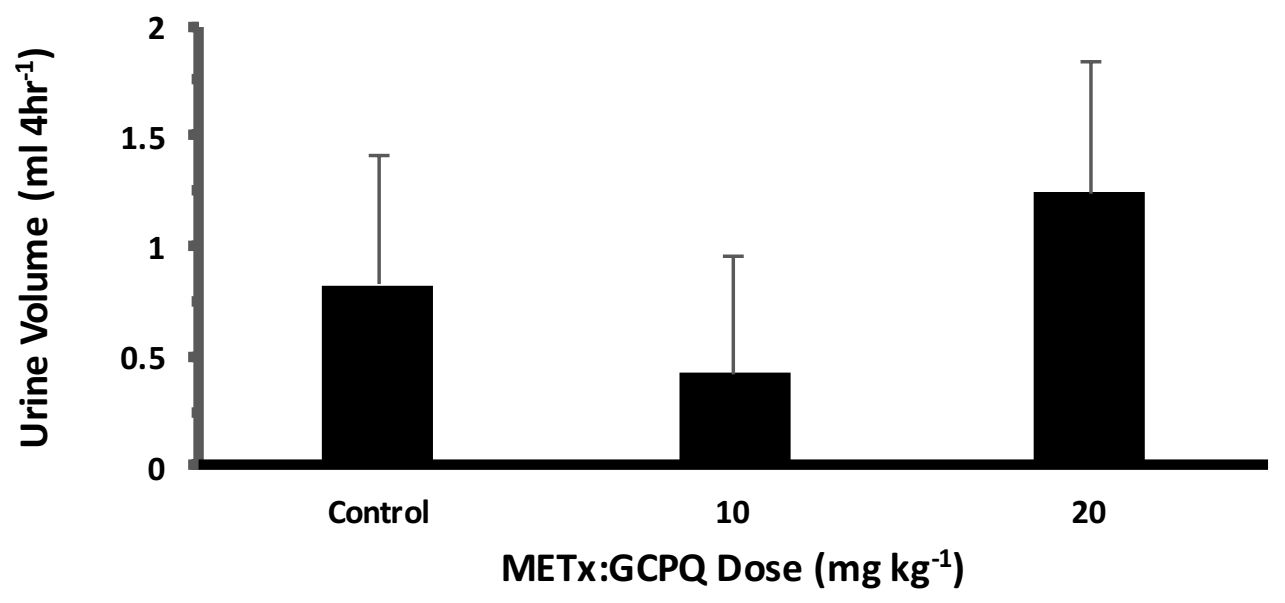

Figure 5.8: Effect of subcutaneous administration METx:GCPQ (1:5) on urine volume production by male SD rats ( $\mathrm{n}=5$ ) over 4 hours, (GCPQ:210115).

To help create a formulation that can be dosed outside of the clinic, the SC route was investigated for any activity. The SC route is currently the main method of delivery of insulin for diabetes mellitus patients. Male SD rats were dosed either GPCQ (control) or METx:GCPQ (1:5) 10 or $20 \mathrm{mg} \mathrm{kg}^{-1}$ subcutaneously in the loose skin around the neck area. After which the animals were placed in individual metabolic cages and spontaneously voided urine was collected for 4 hours. The results are shown in figure 
5.8. There was no difference in the urine produced by either the control or the animals dosed METx.

\subsection{METx:GCPQ (glycerol) Formulation}

\section{Effect of METx:GCPQ (glycerol) on Urine Volume: Subcutaneous Delivery}

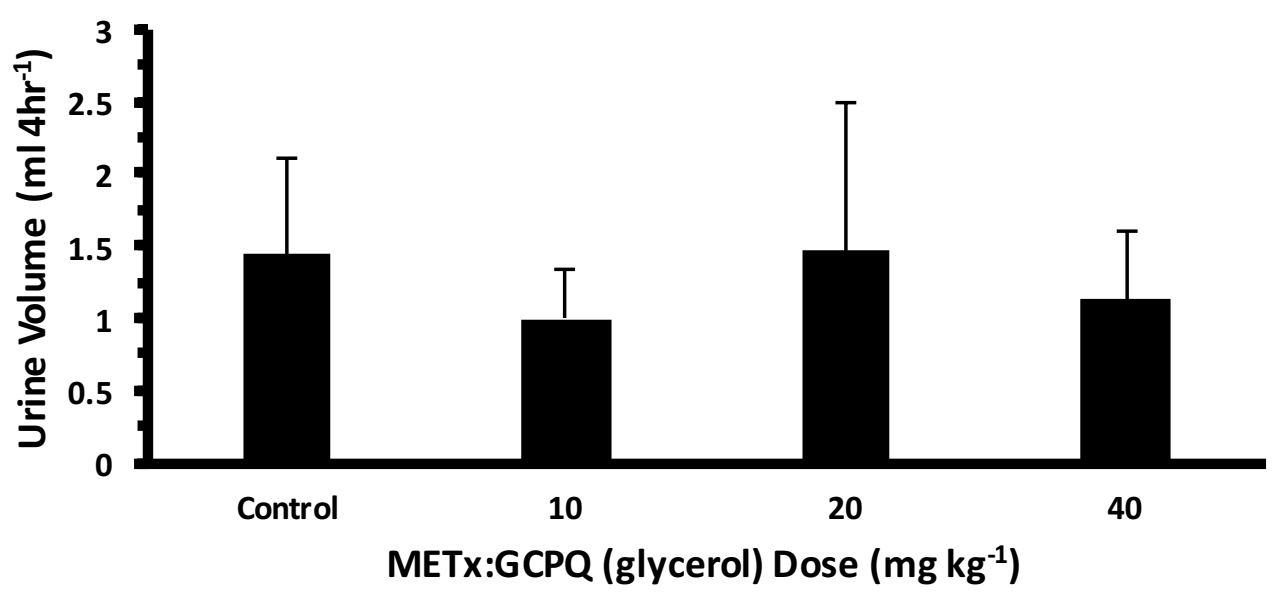

Figure 5.9: Effect of subcutaneous administration METx:GCPQ (glycerol) (1:5) on urine volume production by male SD rats $(\mathrm{n}=5)$ over 4 hours, (GCPQ:090315).

As the METx:GCPQ formulations did not show any activity in the SC or oral route, METx:GCPQ (glycerol) was tried as this would have provided an alternative formulation that may be used outside of the clinic. Male SD rats $(n=5)$ were again dosed subcutaneously in the loose skin behind the neck either control (GCPQ) or METx:GCPQ (glycerol) formulation. After which they were placed individually inside metabolic cages and spontaneously voided urine was collected for 4 hours. The results are shown in figure 5.9. There is no decrease in urine production with those rats dosed 10, 20 or $40 \mathrm{mg} \mathrm{kg}-1$. There is no difference in the urine volume produced by the control or METx dosed rats. This shows that the METx:GCPQ (glycerol) formulation is not active via the SC route.

Both METx:GCPQ and METx:GCPQ (glycerol) formulations were investigated as an alternative to IV delivery of METx. However neither resulted in a decrease in urine production. This shows that these formulations are not able to protect METx 
well enough for it to reach the kidney intact, to have an effect.

\subsubsection{Urine Osmolality}

\section{Effect of METx:GCPQ on Urine Osmolality: Subcutaneous Delivery}

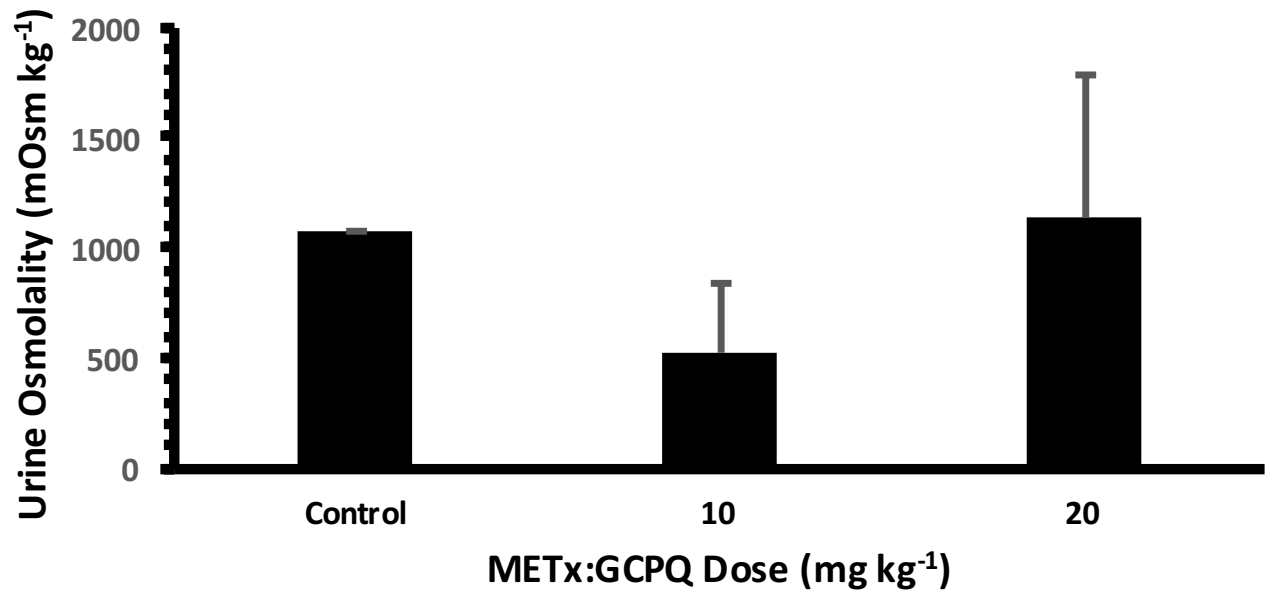

Figure 5.10: Effect of subcutaneous administration METx:GCPQ (1:5) on urine osmolality of urine produced by male SD rats ( $\mathrm{n}=5$ ) over 4 hours, (GCPQ:210115).

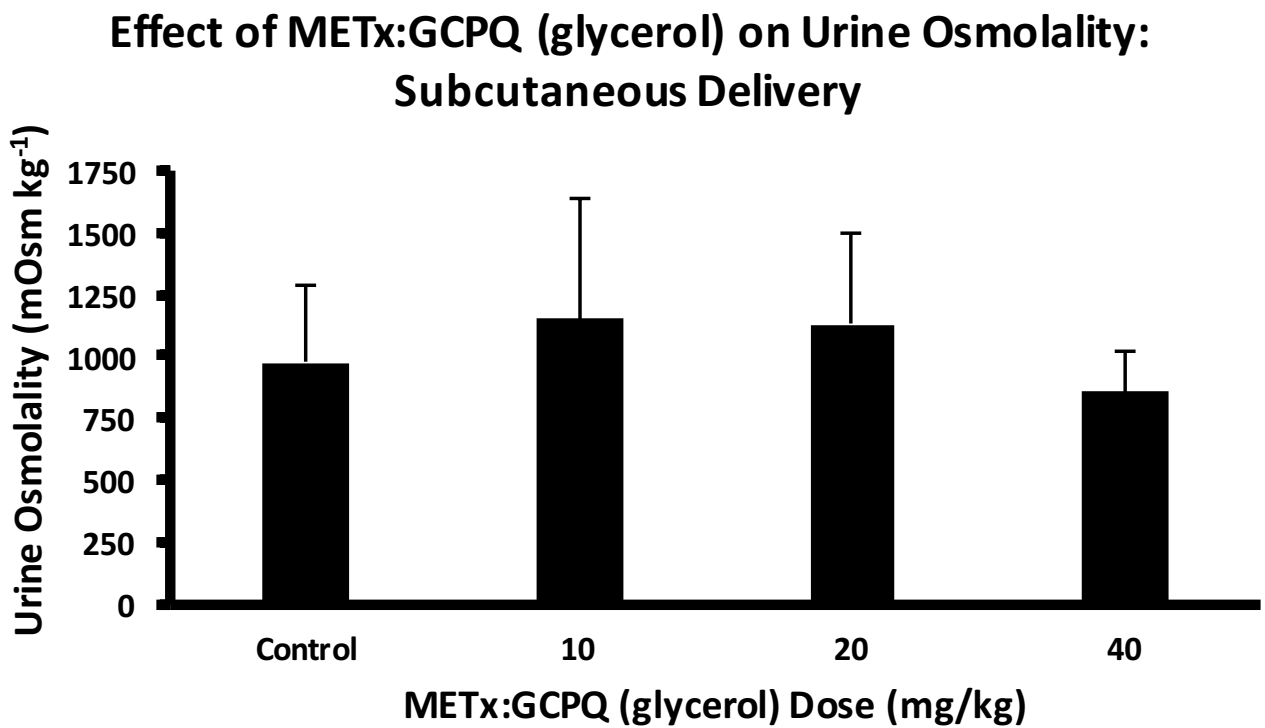

Figure 5.11: Effect of subcutaneous administration METx:GCPQ (glycerol) (1:5) on urine osmolality of urine produced by male SD rats ( $n=5)$ over 4 hours, (GCPQ:090315). 
Even though METx:GCPQ and METx:GCPQ (glycerol) formulations did not have an impact on urine production of the animals, the osmolality of the urine was still measured. If METx was able to concentrate the urine, the osmolality would provide an indication. However, the urine osmolality for both formulations showed no difference compared to the control. This meant that METx was either not reaching the site of action intact or not reaching the site of action at all and was thus not able to influence the volume and concentration of urine. 


\subsection{Discussion}

The function and structure of AVP was first discovered in the 1950s [19] for which Du Vigneaud went on to win the Nobel prize in chemistry. After this many have tried to modify the structure of AVP to create new analogues [23]. We have shown METx to be a partial agonist of the $\mathrm{V}_{2} \mathrm{R}$ receptor and, to test this activity of METx in vivo, urine production studies were performed. The $\mathrm{V}_{2} \mathrm{R}$ is found on the basolateral membrane of the collecting duct of the kidney [267]. Activation of these receptors by AVP results in the insertion of AQP2 water channels into the apical membrane making them permeable to water [26]. A therapeutic agent that has the ability to enhance the antidiuresis of the collecting duct mimicking the actions of AVP may have therapeutic uses in DI, nocturia and NE [35, 407, 415] .

METx was formulated with GCPQ and administered either IV, orally or SC to determine if any of these formulations were able to create a reduction in urine volume. Only the formulation delivered intravenously created a dose-dependent decrease in urine production. The doses administered were 10, 20 and $40 \mathrm{mg} \mathrm{kg}^{-1}$ and compared to animals that had not been dosed at all. There was a decrease in urine production and those rats that had been dosed $40 \mathrm{mg} \mathrm{kg}^{-1}$ did not produce any urine at all. The urine production at $40 \mathrm{mg} \mathrm{kg}^{-1}$ was significantly different to the control animals. The average values for rat urine volume excretion varies from $3.3 \mathrm{~mL}$ per $100 \mathrm{~g}$ bodyweight each day calculated to $8.25 \mathrm{~mL}$ per day for a $250 \mathrm{~g}$ rat [416] whereas others say $23-33 \mathrm{~mL}$ per day per rat [417], therefore it is difficult to compare these results for urine output to an average rat [417].

Comparing these results to other studies; one study found OXT, AVP and desmopressin had be delivered intrathecally to elicit changes in urine output, blood pressure and heart rate. Results found that AVP (3 pM), OXT (300 pM), and desmopressin (30 $\mathrm{pM}$ ) caused a 60-80\% decrease in urine production [418]. Other studies that had taken place to study the effects had been in Battleboro rats, which are rats that lack the ability to produce AVP, found similar results for OXT and AVP urine production [419]. Another study by Kauker et al. to compare the effects of AVP on Long Evans and 
Battleboro rats found that undosed Long Evans rat produced $25 \mu \mathrm{L} \mathrm{min}{ }^{-1} \mathrm{~kg}^{-1}$ which calculates to $1.5 \mathrm{~mL} \mathrm{~min}^{-1}$ for a $250 \mathrm{~g}$ rat, comparing this is similar to the undosed rats of this study. The Long-Evan rats that received AVP received it via continuous infusion for 80-90 minutes so the data cannot be compared to this study directly [420].

The osmolality of the urine produced by the rats was determined using freezing point depression. An upward trend was found in the urine osmolality of 10 and 20 $\mathrm{mg} \mathrm{kg}^{-1}$. It would be expected that the urine osmolality should increase with the dose as the similar amount of solutes are being passed within a smaller volume thereby increasing its concentration and so increasing its osmolality. Similar results in urine osmolality increase was reported by Bardoux et al. where urine flow and osmolality was monitored in rats after intraperitoneal (IP) injection of desmopressin. The results showed that as the urine flow decreased an increased in urine osmolality was seen [421].

The same studies were repeated for oral delivery of METx as oral delivery is the most patient accepted form of administering medications [68, 69]. However no difference was seen in urine volume or osmolality at 100 and $200 \mathrm{mg} \mathrm{kg}^{-1}$. We conclude not enough METx is reaching the kidneys to have an action which can mean that the formulation with GCPQ is not protecting the METx enough in the GI tract, not enough METx is reaching past the liver to the systemic circulation and/or it is not remaining stable in the plasma till METx reaches the kidneys. The results from the oral in vivo data do not correlate well with those from the formulation development chapter. Within the formulation development chapter GCPQ based formulations are able to protect METx in gastric (80\%) and intestinal (50\%) mediums. However, this cannot be suggested within the oral in vivo studies, as very little effect can be seen suggesting METx is not protected as well as thought. Previous GCPQ formulations have shown an ability to deliver peptides across the GI tract. GCPQ has been used to deliver LENK to the brain. GCPQ was formulated with LENK in a similar manner to this study and given orally by an oral gavage resulting in increased anti-nociception when compared to LENK alone [139].

Desmopressin, the commercial oral AVP agonist, showed a 50\% reduction in the volume of urine passed $3 \mathrm{hr}$ after oral administration in a clinical trial [12]. Although 
desmopressin has such a distinct effects its oral bioavailability is variable and low with studies showing between $0.08-0.1 \%$ [422]. Similar results of low bioavailability were found in rats, $0.03 \%$ [423]. Direct comparisons of these cannot be made to METx as desmopressin is known to have a far greater potency than AVP and so a much lower amount would be needed to have a therapeutic effect.

A similar urine volume and urine osmolality experiment was performed, as SC injections are more favoured than IV injections by both the patients and health care professionals [101, 104]. METx was tested subcutaneously with GCPQ and GCPQ (glycerol) formulations however neither produced any difference in production of urine volume or urine osmolality. The lack of activity shows that not enough METx was reaching the site of action to have an effect. Although delivery of peptides subcutaneously provides a means to bypass the barrier of degrading enzymes of the GI tract, transport thorough the SC tissue into the systemic circulation still does not mean complete bioavailability [105]. Studies have shown that variable amounts of peptides and proteins cross the SC tissue; one study found that $35 \%$ insulin crosses the SC tissue into systemic circulation [121]. Another study reported this value to be around 65\% [424]. Similar results were also reported in other studies, however SC delivery offers a higher bioavailability and hence a larger chance of effect compared to oral peptide delivery [425].

METx has shown an ability to reduce urine volume upon administration. Currently in the clinic the main molecule used for a similar effect is desmopressin. Although desmopressin is more potent than AVP and is generally well tolerated there are requirements for with a strict fluid diet which can have an impact on day to day activities [60]. METx as partial agonist may provide an alternative if a suitable oral formulation can be developed. 


\section{Chapter 6: Conclusions \& Future Work}

Desmopressin has become the main stay for treatment of DI [54]. Although it is well tolerated in most patients, there is a need for strict fluid intake, as day to day variability can lead to fluid intoxication [60]. Recently desmopressin as a $\mathrm{V}_{2} \mathrm{R}$ agonist has received greater attention for the treatment of other conditions such as NE, polyuria and enuresis. Within this study METx was developed as an alternative to desmopressin for conditions where an AVP analogue would provide treatment. Previous experiments of treating DI patients with OXT found that OXT has a similar but less potent effect to that of desmopressin [426].

METx is a hydrophobic derivate, where the addition of palmitoyl chain makes it insoluble in water. Some peptide characterisation studies were performed to identify the CMC of METx and its solubility. METx was found to have a CMC of $27.4 \mathrm{mg}$ $\mathrm{L}^{-1}(20 \mu \mathrm{M})$. METx showed higher solubility in organic solvents, with the highest solubility in DMF $\left(833 \mu \mathrm{g} \mathrm{ml}^{-1}\right)$. Further experiments that could be performed such as calculating pka and whether METx is amorphous or crystalline; these experiments will help to understand the properties of METx more. The pKa of METx would be helpful as it can help predict its lipophilicity and permeability at different pHs thereby helping to improve its absorption, thus helping to maximise the effect of METx [427].

The next experiments helped to understand METx's activity on the $V_{2} R$ by monitoring cAMP production. METx was found to be a partial agonist of the $V_{2} R$, this was similar to what other studies had found about OXT on the $\mathrm{V}_{2} \mathrm{R}$ [323]. Further in vitro experiments would need to be performed to further understand METx's selectivity for AVP receptors, as one of the biggest challenges is to develop selective agonists [22]. The AVP receptors are very closed related to the OXT receptors, sharing $40 \%$ 
of the same amino acid sequence of their receptors [428]. Each of the AVP and OXT receptors has a different function within the body.

One of the reasons desmopressin has become such an important treatment options is due to its selectivity. Desmopressin does not show any affinity for the $V_{1 A} R$, thereby reducing its vasoconstriction side effects [429]. However further studies found that desmopressin was a more $\mathrm{V}_{1 \mathrm{~B}} \mathrm{R}$ agonist than $\mathrm{V}_{2} \mathrm{R}$ agonist in humans, but in rats desmopressin is a more potent $\mathrm{V}_{2} \mathrm{R}$ agonist than $\mathrm{V}_{1 \mathrm{~B}} \mathrm{R}$ agonist and a lesser extent to the OXT receptors [47, 428]. Currently desmopressin is the only $\mathrm{V}_{2} \mathrm{R}$ agonist available, attempts for development of other analogues led to development of terlipressin, a $\mathrm{V}_{1 \mathrm{~A}} \mathrm{R}$ agonist [45].

The $\mathrm{V}_{1 \mathrm{~A}} \mathrm{R}$ and $\mathrm{V}_{1 \mathrm{~B}} \mathrm{R}$ activity of METx may be measured either by monitoring intracellular movement of $\mathrm{Ca}^{2+}$ or $\mathrm{IP}_{3}$, its secondary messenger. An improvement on the use of primary cell lines such as MDCK is to transfect Chinese hamster ovary (CHO) or human embryonic kidney (HEK) cells with the human and rat form of the receptors. This again would provide some useful data as the human and rat AVP receptors differ slightly, the importance of this has been exemplified in desmopressin studies discussed above.

Formulation development involved using GCPQ to formulate METx for IV, oral and SC delivery, a ratio of METx:GCPQ (1:5, w/w) was found to be most stable in plasma, SGF and RIW and for the METx:GCPQ (glycerol) the same ratio 1:5 (w/w) was found to be the most stable in plasma. The METx:GCPQ (glycerol) formulation was found to be stable for 14 days when stored in the fridge. It however, degraded quickly when stored at room temperature, with METx content reducing after 2 days.

In vivo studies were performed to see if the agonist activity of METx could be seen in animals, this also provided an opportunity to test the strength of METx:GCPQ formulation in protecting METx. METx showed antidiuretic activity when delivered IV. METx produced a dose responsive effect when give by IV injection, with those animal dosed $40 \mathrm{mg} \mathrm{kg}^{-1}$ unable to produce any urine at all. METx was not able to provide the same effect when administered orally or SC injection. 
Oral administration of medications is the most preferred route by all patients. Another reason desmopressin has been main stay treatment as it has all the benefits of being a peptide [68, 69]. Desmopressin can be taken at home at patients own connivence. Although desmopressin has very low bio availability $(0.1 \%)$ [422], this is enough for it to have the therapeutic effect required. Chemical modification has meant that desmopressin is very stable in GI fluids and plasma [385, 430, 431]. METx does not currently have the ability to be delivered in a non clinical environment, for this to become the case some further work will be required. METx did not show activity when administered orally or SC.

Formulation development would be required to improve the delivery of METx. Although an optimal ratio of METx:GCPQ has been investigated here, this has not resulted in systemic delivery of METx. METx produced an effect when delivered by the IV route, this means it is active in vivo and requires a better delivery system. Many of the oral peptide clinical trials taking place use a permeation enhancer technology for the oral delivery of peptides such as insulin [145], so the introduction of a permeation enhancer could be tested. This would also help to understand if stability or absorption is the limiting factor in oral delivery of METx:GCPQ. Stability studies have shown METx is protected by GCPQ from the harsh GI tract and plasma, permeation enhancers would assist in transport of METx from site of administration to the systemic circulation. Other options are to test various other co-solvents. Recently lomustine has been formulated with GCPQ, soy bean oil and polysorbate for brain cancer delivery [432]. To this effect more formulation studies would be performed with a range of excipients to aid delivery. To improve the storage stability of METx:GCPQ (glycerol) the breakdown could be studied by MS, this may help to find other methods to help improve the stability of the freeze-dried formulations.

Aside from understanding and delivering METx future experiments could also involve some structure activity experiments to increase METx potency and selectivity. Desmopressin has two modifications deamination of Cys at position 1, which improves its half life and enhances the antidiuretic activity, and the substitution at position 8 from L-Arg to D-Arg, results in loss of activity at the $\mathrm{V}_{1 \mathrm{~A}} \mathrm{R}$ [433]. Similar modifications can be tested on METx to see if this would improve its properties too. However no pa- 
pers could be found where OXT structure has been studied to improve its antidiuretic activity. 


\section{Bibliography}

[1] K. Lintner, "Peptides and proteins," in Cosmetic Dermatology, ch. 36, pp. 292301, Oxford, UK: Wiley-Blackwell.

[2] A. B. Hughes, Amino acids, peptides, and proteins in organic chemistry. WileyVCH, 2009.

[3] K. Fosgerau and T. Hoffmann, "Peptide therapeutics: Current status and future directions," Drug Discovery Today, vol. 20, no. 1, pp. 122-128, 2015.

[4] C. M. Edwards, M. A. Cohen, and S. R. Bloom, "Peptides as drugs.," QJM : monthly journal of the Association of Physicians, vol. 92, pp. 1-4, jan 1999.

[5] D. J. Craik, D. P. Fairlie, S. Liras, and D. Price, "The Future of Peptide-based Drugs," Chemical Biology \& Drug Design, vol. 81, no. 1, pp. 136-147, 2013.

[6] L. Sun, "Peptide-Based Drug Development," Modern Chemistry \& Applications, vol. 1, no. 1, pp. 1-2, 2013.

[7] J. Renukuntla, A. D. Vadlapudi, A. Patel, S. H. Boddu, and A. K. Mitra, “Approaches for enhancing oral bioavailability of peptides and proteins," International Journal of Pharmaceutics, vol. 447, no. 1-2, pp. 75-93, 2013.

[8] B. Leader, Q. J. Baca, and D. E. Golan, "Protein therapeutics: a summary and pharmacological classification.," Nature reviews. Drug discovery, vol. 7, no. 1, pp. 21-39, 2008.

[9] B. L. Bray, "Large-scale manufacture of peptide therapeutics by chemical synthesis.," Nature reviews. Drug discovery, vol. 2, no. 7, pp. 587-593, 2003. 
[10] M. Goldberg and I. Gomez-Orellana, "Challenges for the oral delivery of macromolecules.," Nature reviews. Drug discovery, vol. 2, no. 4, pp. 289-295, 2003.

[11] L. R. Brown, “Commercial challenges of protein drug delivery.," Expert opinion on drug delivery, vol. 2, no. 1, pp. 29-42, 2005.

[12] A. A. Kaspar and J. M. Reichert, "Future directions for peptide therapeutics development," Drug Discovery Today, vol. 18, no. 17-18, pp. 807-817, 2013.

[13] PR Newsire, "Global Peptide Therapeutics Market to Value USD 25.4 Billion by 2018: Transparency Market Research,” 2014.

[14] F. Albericio and H. G. Kruger, "Therapeutic peptides," Future Medicinal Chemistry, vol. 4, no. 12, pp. 1527-1531, 2012.

[15] D. M. Ecker, S. D. Jones, and H. L. Levine, "The therapeutic monoclonal antibody market," $m A b s$, vol. 7, no. 1, pp. 9-14, 2015.

[16] P. Vlieghe, V. Lisowski, J. Martinez, and M. Khrestchatisky, "Synthetic therapeutic peptides: science and market," Drug Discovery Today, vol. 15, no. 1-2, pp. 40-56, 2010.

[17] G. Oliver and E. A. Schäfer, "On the Physiological Action of Extracts of Pituitary Body and certain other Glandular Organs: Preliminary Communication.," The Journal of Physiology, vol. 18, pp. 277-9, jul 1895.

[18] H. H. Dale, "The Action of Extracts of the Pituitary Body.," The Biochemical Journal, vol. 4, no. 9, pp. 427-47, 1909.

[19] V. du Vigneaud, D. T. Gish, and P. G. Katsoyannis, "A Synthetic Preparation Possessing Biological Properties Assocaited with Arginine Vasopressin," Journal of the American Chemical Society, vol. 76, pp. 4751-4752, sep 1954.

[20] V. DU VIGNEAUD, C. RESSLER, and S. TRIPPETT, “The sequence of amino acids in oxytocin, with a proposal for the structure of oxytocin.," The Journal of biological chemistry, vol. 205, pp. 949-57, dec 1953.

[21] W. Sneader, "Chapter 14: Peptide Hormones," in Drug discovery : a history, Wiley, 2005. 
[22] M. Manning, S. Stoev, B. Chini, T. Durroux, B. Mouillac, and G. Guillon, “Peptide and non-peptide agonists and antagonists for the vasopressin and oxytocin $\mathrm{V} 1 \mathrm{a}, \mathrm{V} 1 \mathrm{~b}, \mathrm{~V} 2$ and OT receptors : research tools and potential therapeutic agents," Progress in Brain Research, vol. 170, no. 08, pp. 473-512, 2008.

[23] M. Manning, A. Misicka, A. Olma, K. Bankowski, S. Stoev, B. Chini, T. Durroux, B. Mouillac, M. Corbani, and G. Guillon, "Oxytocin and Vasopressin Agonists and Antagonists as Research Tools and Potential Therapeutics Neuroendocrinology," Journal of Neuroendocrinology, no. 23, pp. 609-628, 2012.

[24] G. Gimpl, "Oxytocin receptor ligands: a survey of the patent literature," Expert Opinion on Therapeutic Patents, vol. 18, no. 11, pp. 1239-1251, 2008.

[25] G. Gimpl, F. Fahrenholz, and C. Gene, "The Oxytocin Receptor System : Structure , Function , and Regulation,” Physiological Reviews, vol. 81, no. 2, pp. 629$683,2001$.

[26] M. Thibonnier, P. Coles, A. Thibonnier, and M. Shoham, "The basic and clinical pharmacology of nonpeptide vasopressin receptor antagonists.," Annual review of pharmacology and toxicology, vol. 41, pp. 175-202, 2001.

[27] H. H. Zingg, "Vasopressin and oxytocin receptors," Bailliere's Clinical Endocrinology and Metabolism, vol. 10, no. 1, pp. 75-96, 1996.

[28] B. Chini, B. Mouillac, Y. Ala, M. N. Balestre, S. Trumpp-Kallmeyer, J. Hoflack, J. Elands, M. Hibert, M. Manning, and S. Jard, "Tyr115 is the key residue for determining agonist selectivity in the V1a vasopressin receptor.," The EMBO journal, vol. 14, no. 10, pp. 2176-2182, 1995.

[29] M. Thibonnier, P. Coles, A. Thibonnier, and M. Shoham, "Molecular pharmacology and modeling of vasopressin receptors," Progress in Brain Research, vol. 139, pp. 179-196, 2002.

[30] R. W. Lehrich and A. Greenberg, "When is it appropriate to use vasopressin receptor antagonists?," Journal of the American Society of Nephrology : JASN, vol. 19 , no. 6 , pp. 1054-8, 2008. 
[31] T. Koshimizu, K. Nakamura, N. Egashira, M. Hiroyama, H. Nonoguchi, and A. Tanoue, "Vasopressin V1a and V1b Receptors: From Molecules to Physiological Systems," Physiological Reviews, vol. 92, pp. 1813-1864, oct 2012.

[32] F. Ali, M. Guglin, P. Vaitkevicius, and J. K. Ghali, "Therapeutic potential of vasopressin receptor antagonists.," Drugs, vol. 67, no. 6, pp. 847-58, 2007.

[33] N. Vrachnis, F. M. Malamas, S. Sifakis, E. Deligeoroglou, and Z. Iliodromiti, "The Oxytocin-Oxytocin Receptor System and Its Antagonists as Tocolytic Agents," vol. 2011, 2011.

[34] H. Thackare, H. D. Nicholson, and K. Whittington, "Oxytocin - Its role in male reproduction and new potential therapeutic uses," Human Reproduction Update, vol. 12, no. 4, pp. 437-448, 2006.

[35] M. Babey, P. Kopp, and G. L. Robertson, "Familial forms of diabetes insipidus: clinical and molecular characteristics," Nature Reviews Endocrinology, vol. 7, no. 12, pp. 701-714, 2011.

[36] K. Kondo, "Recent discovery and development of non-peptide vasopressin V2 receptor agonists," Expert Opinion on Therapeutic Patents, vol. 12, no. 8, pp. 1249-1258, 2002.

[37] X. He, F. Su, F. S. Taccone, R. Laporte, A. L. Kjølbye, J. Zhang, K. Xie, M. D. Moussa, T. M. Reinheimer, and J.-L. Vincent, “A Selective V1A Receptor Agonist, Selepressin, Is Superior to Arginine Vasopressin and to Norepinephrine in Ovine Septic Shock," Critical Care Medicine, vol. 44, no. 1, pp. 23-31, 2016.

[38] M. Thibonnier, "Vasopressin agonists and antagonists.," Hormone research, vol. 34, no. 3-4, pp. 124-128, 1990.

[39] M. Zaoral, J. Kolc, and F. Šorm, "Amino acids and peptides. LXXI. Synthesis of 1-deamino-8-D- $\gamma$-aminobutyrine-vasopressin, 1-deamino-8-Dlysine-vasopressin, and 1-deamino-8-D-arginine-vasopressin," Collection of Czechoslovak Chemical Communications, vol. 32, no. 3, pp. 1250-1257, 1967. 
[40] C. W. Smith and M. F. Ferger, "Synthesis and some pharmacological properties of five analogs of oxytocin having L-homocysteine in position 6.," Journal of Medicinal Chemistry, vol. 19, no. 2, pp. 250-4, 1976.

[41] M. Manning, a. Olma, W. Klis, a. Kolodziejczyk, E. Nawrocka, a. Misicka, J. Seto, and W. H. Sawyer, "Carboxy terminus of vasopressin required for activity but not binding.," Nature, vol. 308, no. 5960, pp. 652-653, 1984.

[42] P. G. KATSOYANNIS and V. DU VIGNEAUD, “Arginine-vasotocin, a synthetic analogue of the posterior pituitary hormones containing the ring of oxytocin and the side chain of vasopressin.," The Journal of Biological Chemsitry, vol. 233 , no. 6 , pp. $1352-4,1958$.

[43] W. Y. Chan, N. C. Wo, and M. Manning, "The role of oxytocin receptors and vasopressin V1a receptors in uterine contractions in rats: implications for tocolytic therapy with oxytocin antagonists.," American Journal of Obstetrics Gynecology, vol. 175, no. 5, pp. 1331-5, 1996.

[44] S. Stoev, L. L. Cheng, a. Olma, W. a. Klis, M. Manning, W. H. Sawyer, N. C. Wo, and W. Y. Chan, "An investigation of position 3 in arginine vasopressin with aliphatic, aromatic, conformationally-restricted, polar and charged amino acids.," Journal of Peptide Science, vol. 5, no. 3, pp. 141-53, 1999.

[45] A. Kwiatkowska, M. Lewandowska, L. Borovičková, J. Slaninová, B. Lammek, and A. Prahl, "Design, synthesis and structure-activity relationship of new arginine vasopressin analogues containing proline derivatives in position 2.," Chemical Biology \& Drug Designrug design, vol. 81, no. 3, pp. 420-8, 2013.

[46] A. Kwiatkowska, D. Sobolewski, A. Prahl, L. Borovičková, J. Slaninová, and B. Lammek, "Arginine vasopressin and its analogues - The influence of position 2 modification with 3,3-diphenylalanine enantiomers. Highly potent V2 agonists," European Journal of Medicinal Chemistry, vol. 44, no. 7, pp. 2862-2867, 2009.

[47] L. L. Cheng, S. Stoev, M. Manning, S. Derick, A. Pena, M. B. Mimoun, and G. Guillon, "Design of Potent and Selective Agonists for the Human 
Vasopressin V1b Receptor Based on Modifications of [Deamino-Cys1]arginine Vasopressin at Position 4," Journal of Medicinal Chemistry, vol. 47, no. 9, pp. 2375-2388, 2004.

[48] Z. Grzonka, F. Kasprzykowski, E. Kojro, K. Darlak, P. Melin, F. Fahrenholz, P. Crause, and R. Boer, "Arginine-vasopressin analogues with high antidiuretic/vasopressor selectivity. Synthesis, biological activity, and receptor binding affinity of arginine-vasopressin analogues with substitutions in positions 1, 2, 4, 7, and 8.," Journal of Medicinal Chemistry, vol. 29, no. 1, pp. 96-9, 1986.

[49] F. E. Ali, W. Bryan, H. L. Chang, W. F. Huffman, M. L. Moore, G. Heckman, L. B. Kinter, J. McDonald, D. Schmidt, and D. Shue, "Potent vasopressin antagonists lacking the proline residue at position 7.," Journal of Medicinal Chemistry, vol. 29, no. 6, pp. 984-8, 1986.

[50] F. E. Ali, H. L. Chang, W. F. Huffman, G. Heckman, L. B. Kinter, E. F. Weidley, R. Edwards, D. Schmidt, D. Ashton-Shue, and F. L. Stassen, "Potent vasopressin antagonists modified at the carboxy-terminal tripeptide tail.," Journal of Medicinal Chemistry, vol. 30, no. 12, pp. 2291-4, 1987.

[51] B. T. Donovan, The Pituitary Gland, Volumes 1-2. William Clowes and Son, 1966.

[52] N. Back and F. Sicuteri, Vasopeptides Chemistry, Pharmacology, and Pathophysiology. Springer US, 1st ed. ed., 1972.

[53] G. L. Robertson, “Antidiuretic Hormone,” Endocrinology and Metabolism Clinics of North America, vol. 30, no. 3, pp. 671-694, 2001.

[54] N. Di Iorgi, F. Napoli, A. E. M. Allegri, I. Olivieri, E. Bertelli, A. Gallizia, A. Rossi, and M. Maghnie, "Diabetes Insipidus- Diagnosis and Management," Hormone Research in Paediatrics, vol. 77, no. 2, pp. 69-84, 2012.

[55] W. Fenske and B. Allolio, "Clinical review: Current state and future perspectives in the diagnosis of diabetes insipidus: a clinical review.," The Journal of Clinical Endocrinology Metabolism, vol. 97, no. 10, pp. 3426-37, 2012. 
[56] P. H. Baylis and T. Cheetham, "Diabetes insipidus," Archives of Disease in Childhood, vol. 79, no. 1, pp. 84-89, 1998.

[57] D. R. Repaske, R. Medlej, E. K. Gültekin, M. R. Krishnamani, G. Halaby, J. W. Findling, and J. A. Phillips, "Heterogeneity in clinical manifestation of autosomal dominant neurohypophyseal diabetes insipidus caused by a mutation encoding Ala-1->Val in the signal peptide of the arginine vasopressin/neurophysin II/copeptin precursor.," The Journal Of Clinical Endocrinology and Metabolism, vol. 82, no. 1, pp. 51-6, 1997.

[58] S. Ghirardello, C. Malattia, P. Scagnelli, and M. Maghnie, "Current perspective on the pathogenesis of central diabetes insipidus ," Journal of Pediatric Endocrinology and Metabolism, vol. 18, no. 7, pp. 631-645, 2005.

[59] Ferring Pharmaceuticals Ltd, "DDAVP Tablets $0.1 \mathrm{mg}$ Summary of Product Characteristics (SPC) - (eMC)." https://www.medicines.org.uk/emc/medicine/4217\#PHARMACOKINETIC_PROPS, 2011 [Date Accessed: 10 October 2016].

[60] M. Toumba and R. Stanhope, "Morbidity and mortality associated with vasopressin analogue treatment.," Journal of pediatric endocrinology \& metabolism, vol. 19, no. 3, pp. 197-201, 2006.

[61] D. Bockenhauer and D. G. Bichet, "Pathophysiology, diagnosis and management of nephrogenic diabetes insipidus," Nature Publishing Group, vol. 11, no. 1610 , pp. 576-58889, 2015.

[62] S. S. Dychter, D. A. Gold, and M. F. Haller, "Subcutaneous Drug Delivery," Journal of Infusion Nursing, vol. 35, no. 3, pp. 154-160, 2012.

[63] M. Kovalainen, J. Monkare, J. Riikonen, U. Pesonen, M. Vlasova, J. Salonen, V.-P. Lehto, K. Jarvinen, and K.-H. Herzig, "Novel Delivery Systems for Improving the Clinical Use of Peptides," Pharmacological Reviews, vol. 67, no. 3, pp. 541-561, 2015. 
[64] World Health Organistaion, "Adherence to long-term therapies: Evidence for action," European Journal of Cardiovascular Nursing, vol. 2, no. 4, p. 323, 2003.

[65] P. Lindgren, J. Eriksson, M. Buxton, T. Kahan, N. R. Poulter, B. Dahlöf, P. S. Sever, H. Wedel, B. Jönsson, and on behalf of the ASCOT trial investigators, "The economic consequences of non-adherence to lipid-lowering therapy: results from the Anglo-Scandinavian-Cardiac Outcomes Trial," International Journal of Clinical Practice, vol. 64, no. 9, pp. 1228-1234, 2010.

[66] S. Kim, H. Chung, D. Huneidi, and J. Chang, "Barriers of Medication Adherence and Health Outcomes," British Journal of Pharmaceutical Research, vol. 8, no. 5, pp. 1-5, 2015.

[67] F. Braido, I. Baiardini, F. Blasi, R. Pawankar, and G. W. Canonica, “Adherence to asthma treatments," Current Opinion in Allergy and Clinical Immunology, vol. 15, no. 1, pp. 49-55, 2015.

[68] M. D. Dibonaventura, J.-S. Wagner, C. J. Girman, K. Brodovicz, Q. Zhang, Y. Qiu, S.-R. Pentakota, and L. Radican, "Multinational Internet-based survey of patient preference for newer oral or injectable Type 2 diabetes medication.," Patient Preference and Adherence, vol. 4, pp. 397-406, 2010.

[69] A. Shahiwala, "Formulation approaches in enhancement of patient compliance to oral drug therapy.," Expert opinion on drug delivery, vol. 8, no. 11, pp. 15219, 2011.

[70] Markets and Markets, "North American Injectable Drug Delivery Market worth \$16.6 billion by 2017.” 2013, http://www.marketsandmarkets.com/PressReleases/north-america-injectabledrug-delivery.asp [Date Accessed: 10 October 2016].

[71] Markets and Markets, "North American Drug Delivery Technologies Market (Metered Dose Inhalers, Needle-Free Injectors, Auto-Injectors, Nasal Sprays, Transdermal Patches, Nebulizers, Infusion Pumps, Drug Eluting Stents, Sustained Release, Ocular Implants) - Forecasts To 2017." 
2016, http://www.rnrmarketresearch.com/north-american-drug-deliverytechnologies-market-metered-dose-inhalers-needle-free-injectors-autoinjectors-nasal-sprays-transdermal-patches-nebulizers-infusion-pumps-drugeluting-stents-sust-market-report.html [Date Accessed: 10 October 2016].

[72] L. DeVane, "Chapter 8. Principles of Pharmacokinetics and Pharmacodynamicstle," in The American Psychiatric Publishing Textbook of Psychopharmacology, 4th Edition (A. Schatzberg and C. Nemeroff, eds.), ch. Chapter 4, pp. 181200, Washington: American Psychiatric Pub, third edit ed., 2004.

[73] N. N. Salama, N. D. Eddington, and A. Fasano, "Tight junction modulation and its relationship to drug delivery.," Advanced Drug Delivery Reviews, vol. 58, no. 1 , pp. 15-28, 2006.

[74] S. Gupta, A. Jain, M. Chakraborty, J. K. Sahni, J. Ali, and S. Dang, "Oral delivery of therapeutic proteins and peptides: a review on recent developments.," Drug Delivery, vol. 20, no. 6, pp. 237-46, 2013.

[75] A. Maria Calcagno and T. J. Siahaan, "Physiological, Biochemical and Cehmical Barriers to Oral Drug Delivery," in Drug Delivery Principles and Applications (B. Wang, T. J. Siahaan, and R. A. Soltero, eds.), ch. Chapter 2, pp. 15-29, John Wiley \& Sons, 2005.

[76] S. Barua and S. Mitragotri, "Challenges associated with penetration of nanoparticles across cell and tissue barriers: A review of current status and future prospects," Nano Today, vol. 9, no. 2, pp. 223-243, 2014.

[77] E. Blanco, H. Shen, and M. Ferrari, "Principles of nanoparticle design for overcoming biological barriers to drug delivery.," Nature biotechnology, vol. 33, no. 9, pp. 941-951, 2015.

[78] B. F. Choonara, Y. E. Choonara, P. Kumar, D. Bijukumar, L. C. du Toit, and V. Pillay, "A review of advanced oral drug delivery technologies facilitating the protection and absorption of protein and peptide molecules," Biotechnology Advances, vol. 32, no. 7, pp. 1269-1282, 2014. 
[79] U. B. Kompella and V. H. Lee, "Delivery systems for penetration enhancement of peptide and protein drugs: design considerations.," Advanced drug delivery reviews, vol. 46, no. 1-3, pp. 211-45, 2001.

[80] M. Werle and a. Bernkop-Schnürch, "Strategies to improve plasma half life time of peptide and protein drugs.," Amino acids, vol. 30, no. 4, pp. 351-67, 2006.

[81] M. Schenk and C. Mueller, "The mucosal immune system at the gastrointestinal barrier," Best Practice and Research: Clinical Gastroenterology, vol. 22, no. 3, pp. 391-409, 2008.

[82] C. Atuma, V. Strugala, a. Allen, and L. Holm, "The adherent gastrointestinal mucus gel layer: thickness and physical state in vivo.," American journal of physiology. Gastrointestinal and Liver Physiology, vol. 280, no. 5, pp. G922G929, 2001.

[83] L. Montagne, D. Ph, C. Piel, J. P. Lalle, and J. Lalles, "Effect of diet on mucin kinetics and composition: nutrition and health implications," Nutrition reviews, vol. Mar, pp. 105-114, 2004.

[84] M. Martinez, G. Amidon, L. Clarke, W. W. Jones, A. Mitra, and J. Riviere, “Applying the biopharmaceutics classification system to veterinary pharmaceutical products: Part II. Physiological considerations," Advanced Drug Delivery Reviews, vol. 54, no. 6, pp. 825-850, 2002.

[85] G. Fricker and J. Drewe, "Current concepts in intestinal peptide absorption.," Journal of Peptide Science, vol. 2, no. 4, pp. 195-211, 1996.

[86] J. Hochman, "Mechanisms of absorption enhancement and tight junction regulation," Journal of Controlled Release, vol. 29, no. 3, pp. 253-267, 1994.

[87] E. L. Lecluyse, S. C. Sutton, and S. C. Suttonb, "In vitro models for selection of development candidates. Permeability studies to define mechanisms of absorption enhancement," Advanced Drug Delivery Reviews, vol. 23, no. 1-3, pp. 163-183, 1997. 
[88] S. M. Catnach, P. D. Fairclough, and S. M. Hammond, "Intestinal absorption of peptide drugs: advances in our understanding and clinical implications.," Gut, vol. 35, pp. 441-444, 1994.

[89] W. P. Walters, "Going further than Lipinski's rule in drug design," Expert Opinion on Drug Discovery, vol. 7, no. July, pp. 99-107, 2012.

[90] G. B. Santos, A. Ganesan, and F. S. Emery, "Oral Administration of PeptideBased Drugs: Beyond Lipinski’s Rule," ChemMedChem, vol. 11, no. 20, pp. 2245-2251, 2016.

[91] M. Martinez and G. Amidon, “A mechanistic approach to understanding the factors affecting drug absorption: a review of fundamentals," The Journal of Clinical Pharmacology, vol. 42, pp. 620-643, 2002.

[92] J. Pappenheimer and K. Reiss, "Contribution of solvent drag through intercellular junctions to absorption of nutrients by the small intestine of the rat," The Journal of Membrane Biology, vol. 100, no. 2, pp. 123-36, 1987.

[93] G. H. Liang and C. R. Weber, "Molecular aspects of tight junction barrier function," Current Opinion in Pharmacology, vol. 19, pp. 84-89, 2014.

[94] M. Grant and A. Leone-Bay, "Peptide therapeutics: it's all in the delivery.," Therapeutic delivery, vol. 3, no. 8, pp. 981-96, 2012.

[95] R. I. Mahato, A. S. Narang, L. Thoma, and D. D. Miller, "Emerging trends in oral delivery of peptide and protein drugs.," Critical reviews in therapeutic drug carrier systems, vol. 20, no. 2-3, pp. 153-214, 2003.

[96] NICE Guidelines, "Type 1 diabetes in adults: diagnosis and management I Guidance and guidelines I NICE." 2015, https://www.nice.org.uk/guidance/ng17?unlid=382286372016220232952 [Date Accessed: 10 October 2016]. .

[97] R. Visentin, C. Giegerich, R. Jager, R. Dahmen, A. H. Boss, M. Grant, C. Dalla Man, C. Cobelli, and T. Klabunde, "Improving Efficacy of Inhaled Technosphere Insulin (Afrezza $\left.{ }^{\circledR}\right)$ by Post-meal Dosing: In Silico Clinical Trial with 
the UVA/Padova Type 1 Diabetes Simulator," Diabetes Technol Ther, vol. 18, no. 9, pp. 1-12, 2016.

[98] Medscape, "FDA Approves Inhaled Insulin.” 2014, http://www.webmd.com/diabetes/news/20140627/fda-approves-inhaled-insulin [Date Accessed: 10 March 2017]. .

[99] T. A. McDonald, M. L. Zepeda, M. J. Tomlinson, W. H. Bee, and I. A. Ivens, "Subcutaneous administration of biotherapeutics: current experience in animal models.," Current Opinion in Molecular Therapeutics, vol. 12, no. 4, pp. 46170, 2010.

[100] Web MD, "The 10 Most-Prescribed and Top-Selling Medications." 2015, http://www.webmd.com/drug-medication/news/20150508/most-prescribedtop-selling-drugs [Date Accessed: 10 October 2016].

[101] T. Harbo, H. Andersen, A. Hess, K. Hansen, S. H. Sindrup, and J. Jakobsen, "Subcutaneous versus intravenous immunoglobulin in multifocal motor neuropathy: A randomized, single-blinded cross-over trial," European Journal of Neurology, vol. 16, no. 5, pp. 631-638, 2009.

[102] X. Pivot, J. Gligorov, V. Muller, G. Curigliano, A. Knoop, S. Verma, V. Jenkins, N. Scotto, S. Osborne, and L. Fallowfield, "Patients' preferences for subcutaneous trastuzumab versus conventional intravenous infusion for the adjuvant treatment of HER2-positive early breast cancer: final analysis of 488 patients in the international, randomized, two-cohort PrefHer study," Annals of Oncology, vol. 25, no. 10, pp. 1979-1987, 2014.

[103] S. Rule, G. P. Collins, and K. Samanta, "Subcutaneous vs intravenous rituximab in patients with non-Hodgkin lymphoma: A time and motion study in the United Kingdom,” Journal of Medical Economics, vol. 17, no. 7, pp. 459-468, 2014.

[104] J. F. Jin, L. L. Zhu, M. Chen, H. M. Xu, H. F. Wang, X. Q. Feng, X. P. Zhu, and Q. Zhou, "The optimal choice of medication administration route regarding intravenous, intramuscular, and subcutaneous injection," Patient Preference and Adherence, vol. 9, pp. 923-942, 2015. 
[105] W. F. Richter, S. G. Bhansali, and M. E. Morris, "Mechanistic determinants of biotherapeutics absorption following SC administration.," The AAPS journal, vol. 14, no. 3, pp. 559-70, 2012.

[106] W. F. Richter and B. Jacobsen, "Subcutaneous Absorption of Biotherapeutics: Knowns and Unknowns," Drug Metabolism and Disposition, vol. 42, no. 11, pp. 1881-1889, 2014.

[107] K. Aukland and R. K. Reed, "Interstitial-lymphatic mechanisms in the control of extracellular fluid volume.," Physiological reviews, vol. 73, no. 1, pp. 1-78, 1993.

[108] J. E. Scott, "Extracellular matrix, supramolecular organisation and shape.," Journal of Anatomy, vol. 187, no. Pt 2, pp. 259-69, 1995.

[109] H. M. Kinnunen and R. J. Mrsny, "Improving the outcomes of biopharmaceutical delivery via the subcutaneous route by understanding the chemical, physical and physiological properties of the subcutaneous injection site," Journal of Controlled Release, vol. 182, no. 1, pp. 22-32, 2014.

[110] K. Gelse, E. Pöschl, and T. Aigner, "Collagens - Structure, function, and biosynthesis," Advanced Drug Delivery Reviews, vol. 55, no. 12, pp. 1531-1546, 2003.

[111] R. A. Bryant and D. P. Nix, Acute and Chronic Wounds - Elsevieron VitalSource: Current Management Concepts. Mosby-Year Book, 2011.

[112] J. R. Fraser, T. C. Laurent, and U. B. Laurent, "Hyaluronan: its nature, distribution, functions and turnover.," Journal of internal medicine, vol. 242, pp. 27-33, 1997.

[113] F. Horkay, P. Basser, A. Hecht, and E. Geissler, "Chondroitin Sulfate in Solution: Effects of Mono-and Divalent Salts," Macromolecules, vol. 45, no. 6, pp. 2882-2890, 2012.

[114] B. R. Waterhouse and A. D. Farmery, "The organization and composition of body fluids," Anaesthesia and Intensive Care Medicine, vol. 13, no. 12, pp. 603$608,2012$. 
[115] Y. Marunaka, "Roles of interstitial fluid $\mathrm{pH}$ in diabetes mellitus: Glycolysis and mitochondrial function.," World journal of diabetes, vol. 6, no. 1, pp. 125-35, 2015.

[116] N. Fogh-Andersen, B. M. Altura, B. T. Altura, and O. Siggaard-Andersen, "Composition of interstitial fluid," Clinical Chemistry, vol. 41, no. 10, pp. 1522-1525, 1995.

[117] E. W. Ter Braak, J. R. Woodworth, R. Bianchi, B. Cerimele, D. W. Erkelens, J. H. H. Thijssen, and D. Kurtz, "Injection site effects on the pharmacokinetics and glucodynamics of insulin lispro and regular insulin," Diabetes Care, vol. 19, no. 12 , pp. 1437-1440, 1996.

[118] S. A. Beshyah, V. Anyaoku, R. Niththyananthan, P. Sharp, and D. G. Johnston, "The effect of subcutaneous injection site on absorption of human growth hormone: abdomen versus thigh.," Clinical endocrinology, vol. 35, no. 5, pp. 409412, 1991.

[119] M. a. Gibney, C. H. Arce, K. J. Byron, and L. J. Hirsch, "Skin and subcutaneous adipose layer thickness in adults with diabetes at sites used for insulin injections: implications for needle length recommendations.," Current medical research and opinion, vol. 26, no. 6, pp. 1519-30, 2010.

[120] M. a. Swartz, "The physiology of the lymphatic system. [Review] [190 refs]," Advanced Drug Delivery Reviews, vol. 50, no. 1-2, pp. 3-20, 2001.

[121] S. A. Charman, D. N. McLennan, G. A. Edwards, and C. J. H. Porter, "Lymphatic absorption is a significant contributor to the subcutaneous bioavailability of insulin in a sheep model," Pharmaceutical Research, vol. 18, no. 11, pp. 16201626, 2001.

[122] S. Rahimian, M. F. Fransen, J. W. Kleinovink, M. Amidi, F. Ossendorp, and W. E. Hennink, "Polymeric microparticles for sustained and local delivery of antiCD40 and antiCTLA-4 in immunotherapy of cancer," Biomaterials, vol. 61, pp. 33-40, 2015. 
[123] C. Oussoren and G. Storm, "Liposomes to target the lymphatics by subcutaneous administration," Advanced Drug Delivery Reviews, vol. 50, no. 1-2, pp. 143-156, 2001.

[124] A. Supersaxo, W. R. Hein, and H. Steffen, "Effect of molecular weight on the lymphatic absorption of water-soluble compounds following subcutaneous administration.," Pharmaceutical research, vol. 7, no. 2, pp. 167-9, 1990.

[125] L. Kagan, P. Gershkovich, A. Mendelman, S. Amsili, N. Ezov, and A. Hoffman, "The role of the lymphatic system in subcutaneous absorption of macromolecules in the rat model," European Journal of Pharmaceutics and Biopharmaceutics, vol. 67, no. 3, pp. 759-765, 2007.

[126] S. T. Reddy, D. a. Berk, R. K. Jain, and M. a. Swartz, "A sensitive in vivo model for quantifying interstitial convective transport of injected macromolecules and nanoparticles.," Journal of applied physiology, vol. 101, no. 4, pp. 1162-1169, 2006.

[127] M. Patel, M. Boodle, C. Cross, and F. Medical, "Marker to represent intact liposomes in,” Biochimica et Biophysica Acta, vol. 801, pp. 76-86, 1984.

[128] C. D. Kaur, M. Nahar, and N. K. Jain, "Lymphatic targeting of zidovudine using surface-engineered liposomes.," Journal of drug targeting, vol. 16, no. 10, pp. 798-805, 2008.

[129] M. Morishita and N. a. Peppas, "Is the oral route possible for peptide and protein drug delivery?," Drug Discovery Today, vol. 11, no. 19-20, pp. 905-910, 2006.

[130] T. A. Al-Hilal, F. Alam, and Y. Byun, "Oral drug delivery systems using chemical conjugates or physical complexes," Advanced Drug Delivery Reviews, vol. 65, no. 6, pp. 845-864, 2013.

[131] V. P. Torchilin and A. N. Lukyanov, "Peptide and protein drug delivery to and into tumors: Challenges and solutions," Drug Discovery Today, vol. 8, no. 6, pp. 259-266, 2003. 
[132] K. T. Savjani, A. K. Gajjar, and J. K. Savjani, "Drug Solubility: Importance and Enhancement Techniques," ISRN Pharmaceutics, vol. 2012, no. 100 mL, pp. 1-10, 2012.

[133] P. Calceti, S. Salmaso, G. Walker, and a. Bernkop-Schnürch, "Development and in vivo evaluation of an oral insulin-PEG delivery system.," European journal of pharmaceutical sciences, vol. 22, no. 4, pp. 315-23, 2004.

[134] B. J. Bruno, G. D. Miller, and C. S. Lim, "Basics and recent advances in peptide and protein drug delivery," Therapeutic Delivery, vol. 4, no. 11, pp. 1443-1467, 2013.

[135] Y. S. Youn, J. Y. Jung, S. H. Oh, S. D. Yoo, and K. C. Lee, "Improved intestinal delivery of salmon calcitonin by Lys18-amine specific PEGylation: Stability, permeability, pharmacokinetic behavior and in vivo hypocalcemic efficacy," Journal of Controlled Release, vol. 114, no. 3, pp. 334-342, 2006.

[136] H. Vilhardt, "Basic Pharmacology of Desmopressin," Drug Investigation, vol. 2, no. S5, pp. 2-8, 2012.

[137] W. Sawyer, M. Acosta, and M. Manning, "Structural Changes in the Arginine Vasopressin Molecule that Prolong Its Antidiuretic Action," Endocrinology, vol. 95, no. 1, pp. 140-149, 1974.

[138] J. Rautio, H. Kumpulainen, T. Heimbach, R. Oliyai, D. Oh, T. Järvinen, and J. Savolainen, "Prodrugs: design and clinical applications.," Nature reviews. Drug discovery, vol. 7, no. 3, pp. 255-70, 2008.

[139] A. Lalatsa, V. Lee, J. P. Malkinson, M. Zloh, A. G. Schätzlein, and I. F. Uchegbu, "A prodrug nanoparticle approach for the oral delivery of a hydrophilic peptide, leucine5-enkephalin, to the brain," Molecular Pharmaceutics, vol. 9, pp. 1665-1680, 2012.

[140] J. Temsamani and J.-M. Scherrmann, Peptide Transport and Delivery into the Central Nervous System, ch. Peptide vectors as drug carriers, pp. 221-238. Springer US, 2003. 
[141] X. Li, X. Du, J. Li, Y. Gao, Y. Pan, J. Shi, N. Zhou, and B. Xu, "Introducing d amino acid or simple glycoside into small peptides to enable supramolecular hydrogelators to resist proteolysis," Langmuir, vol. 28, no. 37, pp. 13512-13517, 2012.

[142] Z. Feng and B. Xu, "Inspiration from the mirror: D-amino acid containing peptides in biomedical approaches," Biomolecular Concepts, vol. 7, no. 3, pp. 179$187,2016$.

[143] S. Clardy-James, D. G. Allis, T. J. Fairchild, and R. P. Doyle, "Examining the effects of vitamin B12 conjugation on the biological activity of insulin: a molecular dynamic and in vivo oral uptake investigation," MedChemComm, vol. 3, no. 9 , p. $1054,2012$.

[144] S. M. Clardy, D. G. Allis, T. J. Fairchild, and R. P. Doyle, "Vitamin B12 in drug delivery: breaking through the barriers to a B12 bioconjugate pharmaceutical.," Expert opinion on drug delivery, vol. 8, no. 1, pp. 127-40, 2011.

[145] T. A. S. Aguirre, D. Teijeiro-Osorio, M. Rosa, I. S. Coulter, M. J. Alonso, and D. J. Brayden, "Current status of selected oral peptide technologies in advanced preclinical development and in clinical trials," Advanced Drug Delivery Reviews, 2016.

[146] F.-Y. Su, K.-J. Lin, K. Sonaje, S.-P. Wey, T.-C. Yen, Y.-C. Ho, N. Panda, E.Y. Chuang, B. Maiti, and H.-W. Sung, "Protease inhibition and absorption enhancement by functional nanoparticles for effective oral insulin delivery," Biomaterials, vol. 33, no. 9, pp. 2801-2811, 2012.

[147] E. Moroz, S. Matoori, and J. C. Leroux, "Oral delivery of macromolecular drugs: Where we are after almost 100 years of attempts," Advanced Drug Delivery Reviews, vol. 101, pp. 108-121, 2016.

[148] S. Bank, A. Ghosh, S. Bhattacharya, S. Maiti, G. Khan, and A. Sinha, "The control of hyperglycemia by a novel trypsin resistant oral insulin preparation in alloxan induced type i diabetic mice," Scientific Reports, vol. 6, pp. 1-7, 2016. 
[149] V. Agarwal, S. Nazzal, and M. a. Khan, "Optimization and in vivo evaluation of an oral dual controlled-release tablet dosage form of insulin and duck ovomucoid.," Pharmaceutical development and technology, vol. 13, no. 4, pp. 291-8, 2008.

[150] I. Pereira De Sousa and A. Bernkop-Schnürch, "Pre-systemic metabolism of orally administered drugs and strategies to overcome it," Journal of Controlled Release, vol. 192, pp. 301-309, 2014.

[151] L. N. Hassani, A. Lewis, and J. Richard, "Oral Peptide Delivery - Technology Landscape \& Current Status," ONDrugDelivery, vol. 59, pp. 12-17, 2015.

[152] R. Eldor, E. Arbit, A. Corcos, and M. Kidron, "Glucose-Reducing Effect of the ORMD-0801 Oral Insulin Preparation in Patients with Uncontrolled Type 1 Diabetes: A Pilot Study," PLoS ONE, vol. 8, no. 4, pp. 4-7, 2013.

[153] M. A. Karsdal, B. J. Riis, N. Mehta, W. Stern, E. Arbit, C. Christiansen, and K. Henriksen, "Lessons learned from the clinical development of oral peptides," British Journal of Clinical Pharmacology, vol. 79, no. 5, pp. 720-732, 2015.

[154] D. Z. Liu, E. L. LeCluyse, and D. R. Thakker, "Dodecylphosphocholinemediated enhancement of paracellular permeability and cytotoxicity in Caco-2 cell monolayers," Journal of Pharmaceutical Sciences, vol. 88, pp. 1161-1168, nov 1999.

[155] S. Maher and D. J. Brayden, "Overcoming poor permeability: translating permeation enhancers for oral peptide delivery.," Drug discovery today. Technologies, vol. 9, no. 2, pp. e71-e174, 2012.

[156] S. Maher, R. J. Mrsny, and D. J. Brayden, "Intestinal permeation enhancers for oral peptide delivery," Advanced Drug Delivery Reviews, vol. 106, pp. 277-319, 2016.

[157] T. W. Leonard, J. Lynch, M. J. McKenna, and D. J. Brayden, "Promoting absorption of drugs in humans using medium-chain fatty acid-based solid dosage forms: GIPET.," Expert opinion on drug delivery, vol. 3, no. 5, pp. 685-692, 2006. 
[158] J. K. Amory, T. W. Leonard, S. T. Page, E. O’Toole, M. J. McKenna, and W. J. Bremner, "Oral administration of the GnRH antagonist acyline, in a GIPETenhanced tablet form, acutely suppresses serum testosterone in normal men: single-dose pharmacokinetics and pharmacodynamics.," Cancer chemotherapy and pharmacology, vol. 64, pp. 641-5, aug 2009.

[159] L. Boccon-Gibod, E. van der Meulen, and B.-E. Persson, "An update on the use of gonadotropin-releasing hormone antagonists in prostate cancer.," Therapeutic advances in urology, vol. 3, no. 3, pp. 127-140, 2011.

[160] S. Mitragotri, P. A. Burke, and R. Langer, "Overcoming the challenges in administering biopharmaceuticals: formulation and delivery strategies.," Nature reviews. Drug discovery, vol. 13, no. 9, pp. 655-72, 2014.

[161] S. K. Garg, J. B. Buse, J. S. Skyler, D. E. Vaughn, and D. B. Muchmore, "Subcutaneous injection of hyaluronidase with recombinant human insulin compared with insulin lispro in type 1 diabetes.," Diabetes, obesity \& metabolism, vol. 16, no. 11, pp. 1065-1069, 2014.

[162] Novo Nordisk, "Novo Nordisk announces positive results for phase 2 trial with oral semaglutide in people with type 2 diabetes." 2015, https://www.novonordisk.com/bin/getPDF.1896081.pdf [Date Accessed: 10 October 2016] .

[163] M. Kristensen and H. M. Nielsen, "Cell-Penetrating Peptides as Carriers for Oral Delivery of Biopharmaceuticals," Basic and Clinical Pharmacology and Toxicology, vol. 118, no. 2, pp. 99-106, 2016.

[164] L. Vasconcelos, K. Pärn, and U. Langel, "Therapeutic potential of cellpenetrating peptides," Future Medicinal Chemistry, vol. 4, no. 5, pp. 573-591, 2013.

[165] S. El-Andaloussi, T. Holm, and U. Langel, "Cell-penetrating peptides: mechanisms and applications.," Current pharmaceutical design, vol. 11, no. 28, pp. 3597-611, 2005. 
[166] X. Zhu, W. Shan, P. Zhang, Y. Jin, S. Guan, T. Fan, Y. Yang, Z. Zhou, and Y. Huang, "Penetratin derivative-based nanocomplexes for enhanced intestinal insulin delivery," Molecular Pharmaceutics, vol. 11, no. 1, pp. 317-328, 2014.

[167] F. Duchardt, M. Fotin-Mleczek, H. Schwarz, R. Fischer, and R. Brock, “A comprehensive model for the cellular uptake of cationic cell-penetrating peptides," Traffic, vol. 8, no. 7, pp. 848-866, 2007.

[168] M. Kristensen, H. Franzyk, M. T. Klausen, A. Iversen, J. S. Bahnsen, R. B. Skyggebjerg, V. Foderà, and H. M. Nielsen, "Penetratin-Mediated Transepithelial Insulin Permeation: Importance of Cationic Residues and $\mathrm{pH}$ for Complexation and Permeation," The AAPS Journal, vol. 17, no. 5, pp. 12001209, 2015.

[169] E.-S. Khafagy and M. Morishita, "Oral biodrug delivery using cell-penetrating peptide," Advanced Drug Delivery Reviews, vol. 64, no. 6, pp. 531-539, 2012.

[170] M. Morishita, N. Kamei, J. Ehara, K. Isowa, and K. Takayama, “A novel approach using functional peptides for efficient intestinal absorption of insulin," Journal of Controlled Release, vol. 118, pp. 177-184, apr 2007.

[171] E.-S. Khafagy, M. Morishita, N. Kamei, Y. Eda, Y. Ikeno, and K. Takayama, "Efficiency of cell-penetrating peptides on the nasal and intestinal absorption of therapeutic peptides and proteins.," International journal of pharmaceutics, vol. 381, no. 1, pp. 49-55, 2009.

[172] A. K. Bajpai, S. K. Shukla, S. Bhanu, and S. Kankane, "Responsive polymers in controlled drug delivery," Progress in Polymer Science, vol. 33, no. 11, pp. 1088-1118, 2008.

[173] K. Chaturvedi, K. Ganguly, M. N. Nadagouda, and T. M. Aminabhavi, "Polymeric hydrogels for oral insulin delivery.," Journal of Controlled Release, vol. 165, no. 2, pp. 129-38, 2013.

[174] L. Yin, J. Ding, L. Fei, M. He, F. Cui, C. Tang, and C. Yin, "Beneficial properties for insulin absorption using superporous hydrogel containing interpen- 
etrating polymer network as oral delivery vehicles.," International Journal of Pharmaceutics, vol. 350, no. 1-2, pp. 220-9, 2008.

[175] M. González-Alvarez, I. González-Alvarez, and M. Bermejo, "Hydrogels: an interesting strategy for smart drug delivery.," Therapeutic delivery, vol. 4, no. 2, pp. 157-60, 2013.

[176] P. Mukhopadhyay, K. Sarkar, S. Bhattacharya, A. Bhattacharyya, R. Mishra, and P. P. Kundu, "PH sensitive N-succinyl chitosan grafted polyacrylamide hydrogel for oral insulin delivery," Carbohydrate Polymers, vol. 112, pp. 627-637, 2014.

[177] P. Couvreur and F. Puisieux, "Nanoparticles for the Delivery of Peptides and Proteins," in Targeting of Drugs 4, pp. 153-159, Boston, MA: Springer US, 1994.

[178] E. E. P. Herrero, M. M. J. Alonso, and N. Csaba, "Polymer-based oral peptide nanomedicines," Therapeutic Delivery, vol. 3, pp. 657-668, 2012.

[179] J. W. Joseph, J. Kalitsky, S. St-Pierre, and P. L. Brubaker, "Oral delivery of glucagon-like peptide-1 in a modified polymer preparation normalizes basal glycaemia in diabetic db/db mice," Diabetologia, vol. 43, no. 10, pp. 1319$1328,2000$.

[180] K. S. Soppimath, T. M. Aminabhavi, a. R. Kulkarni, and W. E. Rudzinski, "Biodegradable polymeric nanoparticles as drug delivery devices.," Journal of Controlled Release, vol. 70, no. 1-2, pp. 1-20, 2001.

[181] A. Patel, M. Patel, X. Yang, A. K. Mitra, and K. City, "Special Emphasis on Polymeric Nanoparticles," Protein Peptide Letters, vol. 21, no. 11, pp. 11021120, 2015.

[182] A. Siew, H. Le, M. Thiovolet, P. Gellert, A. Schatzlein, and I. Uchegbu, "Enhanced oral absorption of hydrophobic and hydrophilic drugs using quaternary ammonium palmitoyl glycol chitosan nanoparticles," Molecular Pharmaceutics, vol. 9, no. 1, pp. 14-28, 2012. 
[183] A. Lalatsa, N. L. Garrett, T. Ferrarelli, J. Moger, A. G. Schätzlein, and I. F. Uchegbu, "Delivery of peptides to the blood and brain after oral uptake of quaternary ammonium palmitoyl glycol chitosan nanoparticles," Molecular Pharmaceutics, vol. 9, pp. 1764-1774, 2012.

[184] S. Malathi, P. Nandhakumar, V. Pandiyan, T. J. Webster, and S. Balasubramanian, "Novel PLGA-based nanoparticles for the oral delivery of insulin Novel PLGA-based nanoparticles for the oral delivery of insulin," International Journal of Nanomedicine, vol. 10, pp. 2207-2218, 2015.

[185] E. Arbit, M. Goldberg, I. Gomez-Orellana, and S. Majuru, “Oral heparin: status review," Thrombosis Journal, vol. 4, no. 1, p. 6, 2006.

[186] S. Jogala, S. S. Rachamalla, and J. Aukunuru, "Development of subcutaneous sustained release nanoparticles encapsulating low molecular weight heparin.," Journal of advanced pharmaceutical technology \& research, vol. 6, no. 2, pp. 58-64, 2015.

[187] P. Li, H. M. Nielsen, and A. Müllertz, "Oral delivery of peptides and proteins using lipid-based drug delivery systems," Expert Opinion on Drug Delivery, vol. 9, no. 10, pp. 1289-1304, 2012.

[188] S. Jain, V. V. Rathi, A. K. Jain, M. Das, and C. Godugu, "Folate-decorated PLGA nanoparticles as a rationally designed vehicle for the oral delivery of insulin," Nanomedicine, vol. 7, no. 9, pp. 1311-1337, 2012.

[189] R. H. Mu and K. Peters, "Nanosuspensions for the formulation of poorly soluble drugs I . Preparation by a size-reduction technique," vol. 160, pp. 229-237, 1998.

[190] P. Varamini and I. Toth, "Recent advances in oral delivery of peptide hormones," Expert Opinion on Drug Delivery, vol. 5247, no. July, pp. 1-16, 2016.

[191] A. K. Agrawal, D. Urimi, H. Harde, V. Kushwah, and S. Jain, "Folate appended chitosan nanoparticles augment the stability, bioavailability and efficacy of insulin in diabetic rats following oral administration," RSC Advances, vol. 5, no. 127, pp. 105179-105193, 2015. 
[192] J. Li, Y. Wang, L. Han, X. Sun, H. Yu, and Y. Yu, "Time-Action Profile of an Oral Enteric Insulin Formulation in Healthy Chinese Volunteers," Clinical Therapeutics, vol. 34, no. 12, pp. 2333-2338, 2012.

[193] M. L. Immordino, F. Dosio, and L. Cattel, "Stealth liposomes: Review of the basic science, rationale, and clinical applications, existing and potential," International Journal of Nanomedicine, vol. 1, no. 3, pp. 297-315, 2006.

[194] I. P. Kaur, A. Garg, A. K. Singla, and D. Aggarwal, "Vesicular systems in ocular drug delivery: an overview," International Journal of Pharmaceutics, vol. 269, no. 1, pp. 1-14, 2004.

[195] M. Cui, W. Wu, L. Hovgaard, Y. Lu, D. Chen, and J. Qi, "Liposomes containing cholesterol analogues of botanical origin as drug delivery systems to enhance the oral absorption of insulin," International Journal of Pharmaceutics, vol. 489, no. 1-2, pp. 277-284, 2015.

[196] V. P. Torchilin, "Recent advances with liposomes as pharmaceutical carriers.," Nature reviews. Drug discovery, vol. 4, no. 2, pp. 145-160, 2005.

[197] W. B. Geho, H. C. Geho, J. R. Lau, and T. J. Gana, "Hepatic-Directed Vesicle Insulin: A Review of Formulation Development and Preclinical Evaluation," Journal of Diabetes Science and Technology J Diabetes Sci Technol, vol. 33, no. 66, pp. 1451-1459, 2009.

[198] R. J. Y. Ho and M. Gibaldi, “Advanced Drug Delivery,” in Biotechnology and Biopharmaceuticals, pp. 337-380, USA: John Wiley \& Sons, Inc., oct 2004.

[199] Y. Zhang, C. Bachmeier, and D. W. Miller, "In vitro and in vivo models for assessing drug efflux transporter activity," Advanced Drug Delivery Reviews, vol. 55, no. 1, pp. 31-51, 2003.

[200] M. Hashimoto, K. Takada, Y. Kiso, and S. Muranishi, "Synthesis of palmitoyl derivates of insulin and their biological activities," Pharmaceutical Research, vol. 6, no. 2, pp. 171-176, 1989.

[201] S. R. Myers, F. E. Yakubu-Madus, W. T. Johnson, J. E. Baker, T. S. Cusick, V. K. Williams, F. C. Tinsley, A. Kriauciunas, J. Manetta, and V. J. Chen, “Acylation 
of human insulin with palmitic acid extends the time action of human insulin in diabetic dogs," Diabetes, vol. 46, no. 4, pp. 637-642, 1997.

[202] J. Radziuk, S. Pye, B. Bradley, J. Braaten, L. Vignati, P. Roach, R. Bowsher, R. DiMarchi, and R. Chance, "Basal activity profiles of NPH and [Nepsilonpalmitoyl Lys (B29)] human insulins in subjects with IDDM.," Diabetologia, vol. 41, no. 1, pp. 116-20, 1998.

[203] H. Mei, X. C. Yu, and K. K. Chan, "NB1-C16-Insulin: Site-Specific Synthesis, Purification, and Biological Activity," Pharmaceutical Research, vol. 16, no. 11 , pp. 1680-1686, 1999.

[204] R. Gopinath and R. a. S. Naidu, "Pharmaceutical Preformulation Studies - Current Review," International Journal of Pharmaceutical \& Biological Archives, vol. 2, no. 5, pp. 1391-1400, 2011.

[205] M. J. Hageman, "Preformulation designed to enable discovery and assess developability.," Combinatorial chemistry \& high throughput screening, vol. 13, no. 2, pp. 90-100, 2010.

[206] S. S. Bharate and R. a. Vishwakarma, "Impact of preformulation on drug development.," Expert opinion on drug delivery, vol. 10, no. 9, pp. 1239-57, 2013.

[207] ThermoFisher, "Overview of Mass Spectrometry." https://www.thermofisher.com/uk/en/home/life-science/proteinbiology/protein-biology-learning-center/protein-biology-resourcelibrary/pierce-protein-methods/overview-mass-spectrometry.html [Date Accessed: 10 October 2016].

[208] J. H. Gross, "Mass Spectrometry A Textbook," in Mass Spectrometry A Textbook, pp. 1-12, 2004.

[209] G. L. Glish and R. W. Vachet, "The basics of mass spectrometry in the twentyfirst century.," Nature reviews. Drug discovery, vol. 2, no. 2, pp. 140-150, 2003.

[210] M. Yamashita and J. B. Fenn, "Electrospray ion source. Another variation on the free-jet theme," The Journal of Physical Chemistry, vol. 88, no. 20, pp. 44514459, 1984. 
[211] M. Karas, D. Bachmann, U. Bahr, and F. Hillenkamp, "Matrix-assisted ultraviolet laser desorption of non-volatile compounds," International Journal of Mass Spectrometry and Ion Processes, vol. 78, pp. 53-68, 1987.

[212] C. S. Ho, C. W. K. Lam, M. H. M. Chan, R. C. K. Cheung, L. K. Law, L. C. W. Lit, K. F. Ng, M. W. M. Suen, and H. L. Tai, "Electrospray Ionisation Mass Spectrometry : Principles and Clinical Applications," vol. 24, no. February, pp. 3-12, 2003.

[213] A. P. Bruins, "Mechanistic aspects of electrospray ionization," Journal of Chromatography A, vol. 794, no. 1-2, pp. 345-357, 1998.

[214] L. F. Marvin, M. A. Roberts, and L. B. Fay, "Matrix-assisted laser desorption/ionization time-of-flight mass spectrometry in clinical chemistry," Clinica Chimica Acta, vol. 337, no. 1-2, pp. 11-21, 2003.

[215] T. Banerjee, S. Mitra, A. Kumar Singh, R. Kumar Sharma, and A. Maitra, "Preparation, characterization and biodistribution of ultrafine chitosan nanoparticles," International Journal of Pharmaceutics, vol. 243, no. 1-2, pp. 93$105,2002$.

[216] J. K. Lewis, J. Wei, and G. Siuzdak, "Matrix-assisted Laser Desorption / Ionization Mass Spectrometry in Peptide and Protein Analysis," Encyclopedia of Analytical Chemistry, pp. 5880-5894, 2000.

[217] E. De Hoffmann and V. Stroobant, "Introduction," in Mass spectrometry, pp. 110, John Wiley \& Sons, 2007.

[218] R. F. Egerton, Physical Principles of Electron Microscopt An introduction to TEM, SEM and AEM. Springer US, 2005.

[219] D. B. Williams and C. Barry Carter, Transmission Electron Microscopy A textbook for Materila Science. Springer US, 2009.

[220] E. E. Hunter, P. Maloney, and M. Bendayan, Practical electron microscopy : a beginner's illustrated guide. Cambridge University Press, 2nd ed., 2009. 
[221] J. J. Bozzola, "Electron Microscopy," in ENCYCLOPEDIA OF LIFE SCIENCES, pp. 1-10, John Wiley \& Sons, 2002.

[222] E. Calvo, R. Bravo, A. Amigo, and J. Gracia-Fadrique, "Dynamic surface tension, critical micelle concentration, and activity coefficients of aqueous solutions of nonyl phenol ethoxylates," Fluid Phase Equilibria, vol. 282, no. 1, pp. 14-19, 2009.

[223] E. Fuguet, C. Ràfols, M. Rosés, and E. Bosch, "Critical micelle concentration of surfactants in aqueous buffered and unbuffered systems," Analytica Chimica Acta, vol. 548, no. 1-2, pp. 95-100, 2005.

[224] S. Perspicace, A. C. Rufer, R. Thoma, F. Mueller, M. Hennig, S. Ceccarelli, T. Schulz-Gasch, and J. Seelig, "Isothermal titration calorimetry with micelles: Thermodynamics of inhibitor binding to carnitine palmitoyltransferase 2 membrane protein," FEBS Open Bio, vol. 3, pp. 204-211, 2013.

[225] G. Basu Ray, I. Chakraborty, and S. P. Moulik, "Pyrene absorption can be a convenient method for probing critical micellar concentration $(\mathrm{cmc})$ and indexing micellar polarity," Journal of Colloid and Interface Science, vol. 294, no. 1, pp. 248-254, 2006.

[226] C. Wu, N. J. Li, K. C. Chen, and H.-F. Hsu, "Determination of critical micelle concentrations of ionic and nonionic surfactants based on relative viscosity measurements by capillary electrophoresis," Research on Chemical Intermediates, vol. 40, no. 6, pp. 2371-2379, 2014.

[227] J. H. Clint, "Micellization of mixed nonionic surface active agents," Journal of the Chemical Society, Faraday Transactions 1: Physical Chemistry in Condensed Phases, vol. 71, pp. 1327-1334, 1975.

[228] P. R. S. Silva, A. C. Mauro, and C. R. E. Mansur, "Linear and branched polyoxide-based copolymers: Methods to determine the CMC," Journal of Applied Polymer Science, vol. 113, no. 1, pp. 392-399, 2009.

[229] S. F. Burlatsky, V. V. Atrazhev, D. V. Dmitriev, V. I. Sultanov, E. N. Timokhina, E. A. Ugolkova, S. Tulyani, and A. Vincitore, "Surface tension model for sur- 
factant solutions at the critical micelle concentration," Journal of Colloid and Interface Science, vol. 393, no. 1, pp. 151-160, 2013.

[230] A. I. Mitsionis and T. C. Vaimakis, "Estimation of AOT and SDS CMC in a methanol using conductometry, viscometry and pyrene fluorescence spectroscopy methods," Chemical Physics Letters, vol. 547, pp. 110-113, 2012.

[231] T. Inoue, H. Ebina, B. Dong, and L. Zheng, "Electrical conductivity study on micelle formation of long-chain imidazolium ionic liquids in aqueous solution," Journal of Colloid and Interface Science, vol. 314, no. 1, pp. 236-241, 2007.

[232] A. Dominguez, A. Fernandez, N. Gonzalez, E. Iglesias, and L. Montenegro, "Determination of Critical Micelle Concentration of Some Surfactants by Three Techniques," Journal of Chemical Education, vol. 10, no. 10, pp. 1227-1231, 1997.

[233] N. van Os, G. Daane, and G. Haandrikman, "The Effect of Chemical Structure upon the Thermodynamics of Micellization of Model Alkylarenesulfonates III. Determination of the Critical Micelle Concentration and the Enthalpy of Demicellization by Means of Microcalorimetry and a Comparison with the Phase Separation Mode," Journal of Colloid and Interface Science, vol. 141, no. 1, pp. 199-217, 1991.

[234] T. Chakraborty, I. Chakraborty, and S. Ghosh, "The methods of determination of critical micellar concentrations of the amphiphilic systems in aqueous medium," Arabian Journal of Chemistry, vol. 4, no. 3, pp. 265-270, 2011.

[235] K. P. Ananthapadmanabhan, E. D. Goddard, N. J. Turro, and P. L. Kuo, "Fluorescence Probes for Critical Micelle Concentration," Langmuir, vol. 1, no. 3, pp. 352-355, 1985.

[236] U. Anand, C. Jash, and S. Mukherjee, "Spectroscopic determination of Critical Micelle Concentration in aqueous and non-aqueous media using a non-invasive method," Journal of Colloid and Interface Science, vol. 364, no. 2, pp. 400-406, 2011. 
[237] V. R. Meyer, "Introduction," in Pratical High Performance Liquid Chromatography, pp. 1-15, John Wiley \& Sons, fifth ed., 2010.

[238] Y. Kazakevich and R. LoBrutto, "Introduction," in HPLC for Pharmacetuical Scientists (Y. Kazakevich and R. LoBrutto, eds.), ch. Chapter 1, pp. 3-25, John Wiley \& Sons, first ed., 2007.

[239] Agilent Technologies Ltd, "HPLC Basics Fundamentals of Liquid Fundamentals of High Performance Liquid Chromatography (HPLC)." http://polymer.ustc.edu.cn/xwxx_20/xw/201109/P020110906263097048536.pdf [Date Accessed:10 October 2016].

[240] S. D. Kumar and H. Kumar, "Importance of RP-HPLC in Analytical Method Development: A Review," International Journal Of Pharmaceutical Sciences And Research, vol. 3, no. 12, pp. 4626-4633, 2012.

[241] Waters, "How Does High Performance Liquid Chromatography Work? : Waters." http://www.waters.com/waters/en_GB/HowDoes-High-Performance-Liquid-ChromatographyWork\%253F/nav.htm?cid=10049055\&locale=en_GB, [Date Accessed: 10 October 2016].

[242] "Pharmaceutical Applications with HPLC.” http://www.agilent.com/cs/library/applications/59682635.pdf, 2000 [Date Accessed: 10 October 2016]., 2000.

[243] Y. Shi, W. Porter, T. Merdan, and L. C. Li, "Recent advances in intravenous delivery of poorly water-soluble compounds.," Expert opinion on drug delivery, vol. 6, no. 12, pp. 1261-82, 2009.

[244] K. Kovács, T. Orosz, G. Stampf, I. Antal, I. Klebovich, and K. Ludányi, “Difficulties encountered during formulation of a parenteral dosage form containing a poorly soluble drug.," Acta pharmaceutica Hungarica, vol. 79, no. 1, pp. 35-44, 2009.

[245] British Pharmacopoeia Misson, British Pharmacopoeia. London: TSO, 2016. 
[246] M. Aulton and K. Taylor, "Dissolution and solubility," in Aulton's Pharmaceutics, pp. 20-37, 2015.

[247] M. Nic, J. Jirat, and B. Kosata, IUPAC Compendium of Chemical Terminology. USA: IUPAC, 2009.

[248] R. Battino and T. M. Letcher, "An introduction to the understanding of solubility," Journal of Chemical Education, vol. 78, no. 1, p. 103, 2001.

[249] "Chemistry: The Central Science, Chapter 13, Section 3." http://wps.prenhall.com/wps/media/objects/3312/3391718/blb1303.html, [Date Accessed: 10 October 2016].

[250] I. Montes, C. Lai, and D. Sanabria, "Like Dissolves Like: A Classroom Demonstration and a Guided-Inquiry Experiment for Organic Chemistry," Journal of Chemical Education, vol. 80, no. 4, pp. 447-449, 2003.

[251] J. Aguiar, P. Carpena, J. A. Molina-Bolivar, and C. Carnero Ruiz, "On the determination of the critical micelle concentration by the pyrene 1:3 ratio method," Journal of Colloid and Interface Science, vol. 258, no. 1, pp. 116-122, 2003.

[252] J. C. Erve, M. L. Deinzer, and D. J. Reed, "Alkylation of oxytocin by S-(2chloroethyl)glutathione and characterization of adducts by tandem mass spectrometry and Edman degradation.," Chemical research in toxicology, vol. 8, no. 3, pp. 414-21, 1996.

[253] K. J. Weroński, I. Diez-Pérez, M. A. Busquets, C. López-Iglesias, V. Girona, and J. Prat, "Interaction of lipidated GBV-C/HGV NS3 (513-522) and (505514) peptides with phospholipids monolayer. An AFM study," Colloids and Surfaces B: Biointerfaces, vol. 75, no. 1, pp. 25-33, 2010.

[254] C. Hüttl, C. Hettrich, R. Miller, B.-R. Paulke, P. Henklein, H. Rawel, and F. F. Bier, "Self-assembled peptide amphiphiles function as multivalent binder with increased hemagglutinin affinity.," BMC biotechnology, vol. 13, p. 51, 2013.

[255] R. Wakabayashi, Y. Abe, N. Kamiya, M. Goto, N. Kamiya, M. Goto, and M. Goto, "The self-assembly and secondary structure of peptide amphiphiles 
determine the membrane permeation activity," RSC Advances, vol. 4, no. 58, pp. 30654-30657, 2014.

[256] N. M. Javali, A. Raj, P. Saraf, X. Li, and B. Jasti, "Fatty Acid-RGD Peptide Amphiphile Micelles as Potential Paclitaxel Delivery Carriers to $\alpha \mathrm{v} \beta 3$ Integrin Overexpressing Tumors," Pharmaceutical Research, vol. 29, no. 12, pp. 33473361, 2012.

[257] T. Loftsson and M. E. Brewster, "Pharmaceutical applications of cyclodextrins: Basic science and product development," Journal of Pharmacy and Pharmacology, vol. 62, no. 11, pp. 1607-1621, 2010.

[258] V. Koloka, E. D. Christofidou, S. Vaxevanelis, A. A. Dimitriou, V. Tsikaris, A. D. Tselepis, E. Panou-Pomonis, M. Sakarellos-Daitsiotis, and D. C. Tsoukatos, "A palmitoylated peptide, derived from the acidic carboxyl-terminal segment of the integrin $\alpha \mathrm{II} \beta$ cytoplasmic domain, inhibits platelet activation," Platelets, vol. 19, pp. 502-511, jan 2008.

[259] A. Gkourogianni, M. Egot, V. Koloka, V. Moussis, V. Tsikaris, E. PanouPomonis, M. Sakarellos-Daitsiotis, C. Bachelot-Loza, and D. C. Tsoukatos, "Palmitoylated peptide, being derived from the carboxyl-terminal sequence of the integrin $\alpha \mathrm{II} \beta$ cytoplasmic domain, inhibits talin binding to $\alpha \mathrm{II} \beta 3$," Platelets, vol. 25, no. 8, pp. 619-627, 2014.

[260] Y. Wang, A. Lomakin, S. Kanai, R. Alex, and G. B. Benedek, “Transformation of oligomers of lipidated peptide induced by change in $\mathrm{pH}, "$ Molecular Pharmaceutics, vol. 12, no. 2, pp. 411-419, 2015.

[261] FDA, "NDA 206321 SaxendaTM (Liraglutide [rDNA origin] Injection)." http://www.accessdata.fda.gov/drugsatfda_docs/nda/2014/206321Orig1s000ChemR.pdf, 2014 [Date Accessed: 10 October 2016].

[262] S. Hostrup, K. Huus, and H. Parshad, "Modification of Peptides and Proteins," in Delivery Technologies for Biopharmaceuticals, pp. 169-191, Chichester, UK: John Wiley \& Sons, Ltd. 
[263] A. Astashkina, B. Mann, and D. W. Grainger, "A critical evaluation of in vitro cell culture models for high-throughput drug screening and toxicity," Pharmacology \& Therapeutics, vol. 134, pp. 82-106, apr 2012.

[264] N. E. A.-V. Society, "In Testing I Alternatives to Animal Testing and Research." http://www.neavs.org/alternatives/in-testing, 2016 [Date Accessed: 10 October 2016].

[265] T. Hartung, "Food for thought... on cell culture.," ALTEX, vol. 24, no. 3, pp. 143-52, 2007.

[266] T. Hartung and G. Daston, "Are in vitro tests suitable for regulatory use?," Toxicological sciences, vol. 111, no. 2, pp. 233-7, 2009.

[267] J. Robben, N. Knoers, and P. Deen, "Regulation of the Vasopressin V2 Receptor by Vasopressin in Polarized Renal Collecting Duct Cells," Molecular Biology of the Cell, vol. 15, pp. 5693-5699, sep 2004.

[268] D. Latek, A. Modzelewska, B. Trzaskowski, K. Palczewski, and S. Filipek, "G protein-coupled receptors — recent advances," Acta biochimica Polonica, vol. 59, no. 4, pp. 515-529, 2012.

[269] C. D. Sladek and Z. Song, "Diverse Roles of G-Protein Coupled Receptors in the Regulation of Neurohypophyseal Hormone Secretion," Journal of Neuroendocrinology, vol. 24, pp. 554-565, apr 2012.

[270] K. Fushimi, "Phosphorylation of Serine 256 Is Required for cAMP-dependent Regulatory Exocytosis of the Aquaporin-2 Water Channel," Journal of Biological Chemistry, vol. 272, no. 23, pp. 14800-14804, 1997.

[271] J. J. Finley IV, M. A. Konstam, and J. E. Udelson, "Arginine vasopressin antagonists for the treatment of heart failure and hyponatremia," Circulation, vol. 118, no. 4, pp. 410-421, 2008.

[272] M. Boone and P. M. T. Deen, "Physiology and pathophysiology of the vasopressin-regulated renal water reabsorption," Pflugers Archiv European Journal of Physiology, vol. 456, no. 6, pp. 1005-1024, 2008. 
[273] J. Stockland, "Vasopressin Regulation of Renal Sodium Excretion," Kidney International, vol. 78, no. 9, pp. 849-856, 2010.

[274] M. A. Knepper and T. Inoue, "Regulation of aquaporin-2 water channel trafficking by vasopressin," Current Opinion in Cell Biology, vol. 9, no. 4, pp. 560-564, 1997.

[275] S. Mulroney and A. Myers, "Section 5: Renal Physiology," in Netter's Essential Physiology, pp. 202-245, Elsevier Health Sciences, 2015.

[276] R. Zhang and X. Xie, “Tools for GPCR drug discovery.," Acta pharmacologica Sinica, vol. 33, no. 3, pp. 372-84, 2012.

[277] J. Hughes, S. Rees, S. Kalindjian, and K. Philpott, "Principles of early drug discovery," British Journal of Pharmacology, vol. 162, no. 6, pp. 1239-1249, 2011.

[278] W. Thomsen, J. Frazer, and D. Unett, "Functional assays for screening GPCR targets," Current Opinion in Biotechnology, vol. 16, no. 6, pp. 655-665, 2005.

[279] T. D. Pollard, “A guide to simple and informative binding assays.," Molecular biology of the cell, vol. 21, no. 23, pp. 4061-4067, 2010.

[280] L. A. A. De Jong, D. R. A. Uges, J. P. Franke, and R. Bischoff, "Receptor-ligand binding assays: Technologies and applications," Journal of Chromatography B: Analytical Technologies in the Biomedical and Life Sciences, vol. 829, no. 1-2, pp. 1-25, 2005.

[281] E. C. Hulme and M. A. Trevethick, "Ligand binding assays at equilibrium: Validation and interpretation," British Journal of Pharmacology, vol. 161, no. 6, pp. 1219-1237, 2010.

[282] P. Hein, M. C. Michel, K. Leineweber, T. Wieland, N. Wettschureck, and S. Offermanns, "Receptor and Binding Studies," in Practical Methods in Cardiovascular Research, pp. 723-783, 1982. 
[283] A. P. Davenport and F. D. Russell, "Radioligand Binding Assays: Theory and Practice," in Current Directions in Radiopharmaceutical Research and Development, pp. 169-179, Dordrecht: Springer Netherlands, 1996.

[284] T. Kenakin, "Principles: receptor theory in pharmacology.," Trends in pharmacological sciences, vol. 25, no. 4, pp. 186-92, 2004.

[285] S. D. Gan and K. R. Patel, "Enzyme immunoassay and enzyme-linked immunosorbent assay.," The Journal of investigative dermatology, vol. 133, no. 9, p. e12, 2013.

[286] G. B. Wisdom, "Enzyme-immunoassay," Clinical Chemistry, vol. 22, no. 8, pp. 1243-1255, 1976.

[287] R. M. Lequin, "Enzyme immunoassay (EIA)/enzyme-linked immunosorbent assay (ELISA)," Clinical Chemistry, vol. 51, no. 12, pp. 2415-2418, 2005.

[288] S. Deshpande, "Immunoassay Classification and Commercial Technologies," in Enzyme Immunoassays: From Concept to Product Development, pp. 231-274, Springer Science \& Business Media, 2012.

[289] S. Aydin, "A short history, principles, and types of ELISA, and our laboratory experience with peptide/protein analyses using ELISA," Peptides, vol. 72, pp. 4-15, 2015.

[290] Thermo Fisher Scientific Inc, "Overview of ELISA." https://www.thermofisher.com/uk/en/home/life-science/proteinbiology/protein-biology-learning-center/protein-biology-resourcelibrary/pierce-protein-methods/overview-elisa.html\#2, 2015 [Date Accessed: 10 October 2016].

[291] Bio Rad, "Chapter 2: ELISA formats." https://www.bio-radantibodies.com/elisa-types-direct-indirect-sandwich-competition-elisaformats.html, [Date Accessed: 10 October 2016].

[292] K. L. Cox, V. Devanarayan, A. Kriauciunas, J. Manetta, C. Montrose, and S. Sittampalam, Immunoassay Methods. Eli Lilly \& Company and the National Center for Advancing Translational Sciences, 2004. 
[293] L. Kauffman, S. De, L. Llera, S. Angersbach, and M. Orban, "HitHunter TM IP3 Assay for GPCR Screening using the PHERAstar,” 2006.

[294] Amsbio, "General Diacyl Glycerol,DAG/DG ELISA Kit.” http://www.amsbio.com/datasheets/AMS.E2038GE.pdf, [Date Accessed: 10 October 2016].

[295] GE Healthcare Life "Amersham cAMP Biotrak Enzymeimmunoassay (EIA) System.” https://www.gelifesciences.com/gehcls_images/GELS/Related\%20Content /Files/1352308543199/litdoc28953249_20161014013527.pdf, [Date Accessed: 10 October 2016].

[296] A. Tahara, M. Saito, T. Sugimoto, Y. Tomura, K. Wada, T. Kusayama, J. Tsukada, N. Ishii, T. Yatsu, W. Uchida, and a. Tanaka, "Pharmacological characterization of YM087, a potent, nonpeptide human vasopressin V1A and V2 receptor antagonist.," Naunyn-Schmiedeberg's archives of pharmacology, vol. 357, no. 1, pp. 63-9, 1998.

[297] A. L. Del Tredici, K. E. Vanover, A. E. Knapp, S. M. Bertozzi, N. R. Nash, E. S. Burstein, J. Lameh, E. A. Currier, R. E. Davis, M. R. Brann, N. Mohell, R. Olsson, and F. Piu, "Identification of novel selective V2 receptor non-peptide agonists," Biochemical Pharmacology, vol. 76, no. 9, pp. 1134-1141, 2008.

[298] J. W. Gunnet, P. Wines, M. Xiang, P. Rybczynski, P. Andrade-Gordon, L. de Garavilla, T. J. Parry, W.-m. Cheung, L. Minor, K. T. Demarest, B. E. Maryanoff, and B. P. Damiano, "Pharmacological characterization of RWJ676070, a dual vasopressin V1A/V2 receptor antagonist," European Journal of Pharmacology, vol. 590, no. 1-3, pp. 333-342, 2008.

[299] Thermo Fisher Scientific Inc, "Useful Numbers for Cell Culture." https://www.thermofisher.com/uk/en/home/references/gibco-cell-culturebasics/cell-culture-protocols/cell-culture-useful-numbers.html, 2015 [Date Accessed: 10 October 2016].

[300] GE Healthcare Life "Amersham cAMP Biotrak Enzymeimmunoassay (EIA) System.” ht- 
tps://www.gelifesciences.com/gehcls_images/GELS/Related\%20Content /Files/1352308543199/litdoc28953249_20161014013527.pdf, [Date Accessed: 10 October 2016].

[301] R. S. Rittmaster, G. B. J. Cutler, P. W. Gold, D. D. Brandon, T. Tomai, D. L. Loriaux, and G. P. Chrousos, "The relationship of saline-induced changes in vasopressin secretion to basal and corticotropin-releasing hormone-stimulated adrenocorticotropin and cortisol secretion in man.," The Journal of clinical endocrinology and metabolism, vol. 64, no. 2, pp. 371-376, 1987.

[302] A. Sharman and J. Low, "Vasopressin and its role in critical care," Continuing Education in Anaesthesia, Critical Care \& Pain, vol. 8, no. 4, pp. 134-137, 2008.

[303] J.-Y. Goh, R. J. Weaver, L. Dixon, N. J. Platt, and R. A. Roberts, "Development and use of in vitro alternatives to animal testing by the pharmaceutical industry 1980-2013," Toxicol. Res., vol. 4, no. 5, pp. 1297-1307, 2015.

[304] National Centre for Replacement Refinement and Reduction, "The 3Rs." https://www.nc3rs.org.uk/the-3rs, [Date Accessed: 10 October 2016].

[305] European Medicines Agency, "Revised concept paper on the need for revision of the position on the replacement of animal studies by in vitro models." http://www.ema.europa.eu/docs/en_GB/document_library/Scientific_guideline /2012/07/WC500130365.pdf, 2012 [Date Accessed: 10 October 2016].

[306] W. Montague and J. R. Cook, “The role of adenosine 3':5'-cyclic monophosphate in the regulation of insulin release by isolated rat islets of Langerhans.," The Biochemical journal, vol. 122, no. 1, pp. 115-20, 1971.

[307] B. Andersen-Beckh, M. Dehe, R. Schülein, B. Wiesner, C. Rutz, U. Liebenhoff, W. Rosenthal, and A. Oksche, "Polarized expression of the vasopressin V2 receptor in Madin-Darby canine kidney cells," Kidney International, vol. 56, no. 2 , pp. 517-527, 1999.

[308] H. H. Nickols, V. N. Shah, W. J. Chazin, and L. E. Limbird, "Calmodulin interacts with the V2 vasopressin receptor: Elimination of binding to the $\mathrm{C}$ ter- 
minus also eliminates arginine vasopressin-stimulated elevation of intracellular calcium," Journal of Biological Chemistry, vol. 279, no. 45, pp. 46969-46980, 2004 .

[309] A. Tahara, Y. Tomura, K.-i. I. Wada, T. Kusayama, J. Tsukada, M. Takanashi, T. Yatsu, W. Uchida, and a. Tanaka, "Pharmacological Profile of YM087, A Novel Potent Nonpeptide Vasopressin V 1A and V 2 Receptor Antagonist, in Vitro and in Vivo," The Journal of pharmacology and experimental therapeutics, vol. 282, pp. 301-308, jul 1997.

[310] A. Oksche, G. Leder, S. Valet, M. Platzer, K. Hasse, S. Geist, G. Krause, A. Rosenthal, and W. Rosenthal, "Variant amino acids in the extracellular loops of murine and human vasopressin V2 receptors account for differences in cell surface expression and ligand affinity.," Molecular endocrinology (Baltimore, Md.), vol. 16, no. 4, pp. 799-813, 2002.

[311] R. Laporte, A. Kohan, J. Heitzmann, H. Wisniewska, J. Toy, E. La, H. Tariga, S. Alagarsamy, B. Ly, J. Dykert, S. Qi, K. Wisniewski, R. Galyean, G. Croston, C. D. Schteingart, and P. J.-M. Rivière, "Pharmacological characterization of FE 202158, a novel, potent, selective, and short-acting peptidic vasopressin V1a receptor full agonist for the treatment of vasodilatory hypotension.," The Journal of pharmacology and experimental therapeutics, vol. 337, no. 3, pp. 786-96, 2011.

[312] G. Yasuda and W. B. Jeffries, "Regulation of cAMP production in initial and terminal inner medullary collecting ducts," Kidney International, vol. 54, no. 1, pp. 80-86, 1998.

[313] J. A. Lieberman, "Dopamine partial agonists: a new class of antipsychotic.," CNS drugs, vol. 18, no. 4, pp. 251-67, 2004.

[314] K. D. Burris, T. F. Molski, C. Xu, E. Ryan, K. Tottori, T. Kikuchi, F. D. Yocca, and P. B. Molinoff, "Aripiprazole, a novel antipsychotic, is a high-affinity partial agonist at human dopamine D2 receptors.," The Journal of pharmacology and experimental therapeutics, vol. 302, no. 1, pp. 381-389, 2002. 
[315] B. Green, "Focus on aripiprazole.," Current medical research and opinion, vol. 20, no. 2, pp. 207-13, 2004.

[316] W. B. Edwards, C. G. Fields, C. J. Anderson, T. S. Pajeau, M. J. Welch, and G. B. Fields, "Generally applicable, convenient solid-phase synthesis and receptor affinities of octreotide analogs.," Journal of medicinal chemistry, vol. 37, pp. 3749-3757, 1994.

[317] H. W. Lee, M. S. Choo, J. G. Lee, C. H. Park, J. S. Paick, J. Z. Lee, D. H. Han, W. H. Park, and K. S. Lee, "Desmopressin is an effective treatment for mixed nocturia with nocturnal polyuria and decreased nocturnal bladder capacity,” Journal of Korean Medical Science, vol. 25, no. 12, pp. 1792-1797, 2010.

[318] A. Buku, D. Gazis, and I. L. Schwartz, "Antidiuretic and pressor activities of vasopressin analogs with L-alaninamide and D-alaninamide substitutions at position 9.," International journal of peptide and protein research, vol. 23, no. 5, pp. 551-7, 1984.

[319] A. J. Ahmad and E. H. Clark, "Water Intoxication associated with oxytocin infusion," Postgraduate medical journal, vol. 51, pp. 249-252, 1975.

[320] J. G. Pittman, "Water Intoxication Due to Oxytocin,” New England Journal of Medicine, vol. 268, no. 9, pp. 481-482, 1963.

[321] R. R. Potter, "Water Retention due to Oxytocin," Obstetrics \& Gynecology, vol. 23, no. 5, pp. 699-702, 1964.

[322] Y. Terashima, K. Kondo, and Y. Oiso, "Administration of oxytocin affects vasopressin V2 receptor and aquaporin-2 gene expression in the rat.," Life sciences, vol. 64, no. 16, pp. 1447-53, 1999.

[323] J. S. Han, Y. Maeda, and M. a. Knepper, "Dual actions of vasopressin and oxytocin in regulation of water permeability in terminal collecting duct.," The American journal of physiology, vol. 265, no. 1 Pt 2, pp. F26-34, 1993.

[324] C. Li, W. Wang, S. N. Summer, T. D. Westfall, D. P. Brooks, S. Falk, and R. W. Schrier, "Molecular Mechanisms of Antidiuretic Effect of Oxytocin," Journal of the American Society of Nephrology, vol. 19, no. 2, pp. 225-232, 2008. 
[325] B. Berde, W. Doepfner, and H. Konzett, "Some pharmacological actions of four synthetic analogues of oxytocin," British Journal of Pharmacology and Chemotherapy, vol. 12, pp. 209-214, jun 1957.

[326] I. Tsukamoto, "Recent patenting activities in the discovery and development of vasopressin V2 receptor agonists.," Expert opinion on therapeutic patents, vol. 22, no. 6, pp. 579-86, 2012.

[327] J. K. Ghali, H. D. Zmily, J. O. Farah, and S. Daifallah, "Lixivaptan, a nonpeptide vasopressin $\mathrm{V} 2$ receptor antagonist for the potential oral treatment of hyponatremia.," IDrugs : the investigational drugs journal, vol. 13, pp. 78292, nov 2010.

[328] A. a. Failli, J. S. Shumsky, R. J. Steffan, T. J. Caggiano, D. K. Williams, E. J. Trybulski, X. Ning, Y. Lock, T. Tanikella, D. Hartmann, P. S. Chan, and C. H. Park, "Pyridobenzodiazepines: a novel class of orally active, vasopressin V2 receptor selective agonists.," Bioorganic \& medicinal chemistry letters, vol. 16, no. 4, pp. 954-9, 2006.

[329] A. M. Venkatesan, G. T. Grosu, A. A. Failli, P. S. Chan, J. Coupet, T. Saunders, H. Mazandarani, and X. Ru, “(4-Substituted-phenyl)-(5H-10,11-dihydropyrrolo $[2,1-c][1,4]$ benzodiazepin-10-yl)-methanone derivatives as vasopressin receptor modulators," Bioorganic \& Medicinal Chemistry Letters, vol. 15, no. 22 , pp. 5003-5006, 2005.

[330] C. M. Yea, C. E. Allan, D. M. Ashworth, J. Barnett, A. J. Baxter, J. D. Broadbridge, R. J. Franklin, S. L. Hampton, P. Hudson, J. A. Horton, P. D. Jenkins, A. M. Penson, G. R. W. Pitt, P. Rivière, P. A. Robson, D. P. Rooker, G. Semple, A. Sheppard, R. M. Haigh, and M. B. Roe, "New benzylureas as a novel series of potent, nonpeptidic vasopressin V2 receptor agonists," Journal of Medicinal Chemistry, vol. 51, no. 24, pp. 8124-8134, 2008.

[331] J. K. Aronson, "Beta adrenoreceptor antagonists," in Meyler's Side Effects of Cardiovascular Drugs, pp. 37-67, Elsevier, 2009.

[332] S. Frokjaer and D. E. Otzen, "Protein drug stability: a formulation challenge.," Nature reviews. Drug discovery, vol. 4, no. 4, pp. 298-306, 2005. 
[333] Y. Yun, Y. W. Cho, and K. Park, "Nanoparticles for oral delivery: Targeted nanoparticles with peptidic ligands for oral protein delivery," Advanced Drug Delivery Reviews, vol. 65, no. 6, pp. 822-832, 2013.

[334] J. M. Sarciaux, L. Acar, and P. a. Sado, "Using microemulsion formulations for oral drug delivery of therapeutic peptides," International Journal of Pharmaceutics, vol. 120, no. 2, pp. 127-136, 1995.

[335] A. Grenha, "Chitosan nanoparticles: a survey of preparation methods.," Journal of drug targeting, vol. 20, no. 4, pp. 291-300, 2012.

[336] I. Younes and M. Rinaudo, "Chitin and chitosan preparation from marine sources. Structure, properties and applications," Marine Drugs, vol. 13, no. 3, pp. 1133-1174, 2015.

[337] R. Riva, H. Ragelle, and A. des Rieux, Chitosan and chitosan derivatives in drug delivery and tissue engineering. Springer US, 2011.

[338] A. Bernkop-Schnürch and S. Dünnhaupt, "Chitosan-based drug delivery systems," European Journal of Pharmaceutics and Biopharmaceutics, vol. 81, no. 3, pp. 463-469, 2012.

[339] R. Hejazi and M. Amiji, "Chitosan-based gastrointestinal delivery systems," Journal of Controlled Release, vol. 89, no. 2, pp. 151-165, 2003.

[340] M. Thanou, J. C. Verhoef, and H. E. Junginger, "Oral drug absorption enhancement by chitosan and its derivatives," Advanced Drug Delivery Reviews, vol. 52, no. 2 , pp. 117-126, 2001.

[341] M. Werle, H. Takeuchi, and A. Bernkop-Schnürch, "Modified chitosans for oral drug delivery," Journal of Pharmaceutical Sciences, vol. 98, no. 5, pp. 16431656, 2009.

[342] M. Thanou, J. C. Verhoef, J. H. Verheijden, and H. E. Junginger, "Intestinal absorption of octreotide using trimethyl chitosan chloride: studies in pigs.," Pharmaceutical research, vol. 18, no. 6, pp. 823-8, 2001. 
[343] N. Bhattarai, J. Gunn, and M. Zhang, "Chitosan-based hydrogels for controlled, localized drug delivery," Advanced Drug Delivery Reviews, vol. 62, no. 1, pp. 83-99, 2010.

[344] A. Trapani, J. Sitterberg, U. Bakowsky, and T. Kissel, "The potential of glycol chitosan nanoparticles as carrier for low water soluble drugs.," International journal of pharmaceutics, vol. 375, no. 1-2, pp. 97-106, 2009.

[345] I. F. Uchegbu, L. Sadiq, M. Arastoo, A. I. Gray, W. Wang, R. D. Waigh, and A. G. Schätzleinä, "Quaternary ammonium palmitoyl glycol chitosan - A new polysoap for drug delivery," International Journal of Pharmaceutics, vol. 224, no. 1-2, pp. 185-199, 2001.

[346] F. C. B. Solomons Graham T.W, Organic Chemistry. Hoboken: John Wiley \& Sons, 10th editi ed., 2011.

[347] J. Keeler, "Chapter 2: Nuclear spin and spin states," in Understanding NMR Spectroscopy, pp. 2-2-2-21, John Wiley \& Sons, Ltd, 2002.

[348] F. A. Carey, "Chapter 13: Spectroscopy," in Organic Chemsitry, pp. 287-546, 2000.

[349] D. G. Watson, Pharmaceutical Analysis: A Textbook for Pharmacy Students and Pharmaceutical Chemists. London: Churchill Livingstone, third edit ed., 2012.

[350] William "Reusch, "Supplemental NMR Topics." https://www2.chemistry.msu.edu/faculty/reusch/virttxtjml/spectrpy/nmr/nmr2.htm, 2013 [Date Accessed: 10 October 2016]., 2013.

[351] F. C. B. Solomons Graham T.W, Organic Chemistry, 10th Edition. John Wiley \& Sons, 10th editi ed., 2009.

[352] J. Clayden, N. Greeves, and S. Warren, Organic Chemistry. Oxford: OUP, 2nd editio ed., 2012.

[353] A. Lambert, "Review of gel permeation chromatography," British Polymer Journal, vol. 3, no. 1, pp. 13-23, 1971. 
[354] T. Williams, "Gel permeation chromatography: A review," Journal of Materials Science, vol. 5, pp. 811-820, 1970.

[355] Agilent, "An Introduction to Gel Permeation Chromatography and Size Exclusion Chromatography." http://www.scantecnordic.se/Homepage/DownloadFile/f/886526/h/d6bf211199abb0d926102d1741789271/Introduction+to+GPC [Date Accessed: 10 October 2016].

[356] Malvern Instruments, "Static Light Scattering Technologies for GPC/SEC Explained." https://theanalyticalscientist.com/fileadmin/tas/issues/App2013 [Date Accessed:10 October 2016].

[357] W. Technology, "Dn / dc with an Optilab," Light Scattering for the Masses, no. 805 , p. $93117,1999$.

[358] I. F. Uchegbu and A. G. Schätzlein, Polymers in drug delivery. CRC/Taylor \& Francis, 2006.

[359] a. P. Vaidya, R. J. Wigent, J. C. Moore, and J. B. Schwartz, "Protective effect of Carbopol on enzymatic degradation of a peptide-like substrate. I: Effect of various concentrations and grades of Carbopol and other reaction variables on trypsin activity.," Pharmaceutical development and technology, vol. 12, no. 1, pp. 89-96, 2007.

[360] S. M. Moghimi, "Mechanisms of splenic clearance of blood cells and particles: Towards development of new splenotropic agents," Advanced Drug Delivery Reviews, vol. 17, no. 1, pp. 103-115, 1995.

[361] Malvern Instruments, "Dynamic Light Scattering : An Introduction in 30 Minutes." http://www.malvern.com/en/support/resource-center/technicalnotes/TN101104DynamicLightScatteringIntroduction.aspx, [Date Accessed: 10 October 2016].

[362] B. Y. Shekunov, P. Chattopadhyay, H. H. Y. Tong, and A. H. L. Chow, "Particle size analysis in pharmaceutics: principles, methods and applications.," Pharmaceutical research, vol. 24, pp. 203-27, feb 2007. 
[363] Malvern Instruments, "Dynamic Light Scattering Common Terms Defined." $\quad$ http://www.biophysics.bioc.cam.ac.uk/wpcontent/uploads/2011/02/DLS Terms $_{\mathrm{d}}$ efined $_{\mathrm{M}}$ alvern.pdf, 2011[DateAccessed : 10October2016].

[364] A. Iannitelli, A. Godfrey, J. Freeman, A. Schatzlein, and F. Uchegbu, I, "Scale Up of N-palmitoyl-N-monomethyl-N,N-dimethyl-N,N,N-trimethyl-6O-glycolchitosan Production, a New Polymer for Drug Delivery," 2014.

[365] I. Bio-Rad Laboratories, "Quick Start Bradfrod Protien Assay Instruction Manual." http://www.biorad.com/webroot/web/pdf/lsr/literature/4110065A.pdf, [Date Accessed:10 October 2016].

[366] A. T. Florence and D. Attwood, Physicochemical principles of pharmacy : in manufacture, formulation, and clinical use. Pharmaceutical Press, 2005.

[367] V. K. Mourya and N. N. Inamdar, "Chitosan-modifications and applications: Opportunities galore," Reactive and Functional Polymers, vol. 68, no. 6, pp. 1013-1051, 2008.

[368] I. F. Uchegbu, M. Carlos, C. McKay, X. Hou, and A. G. Schätzlein, "Chitosan amphiphiles provide new drug delivery opportunities," Polymer International, vol. 63, no. 7, pp. 1145-1153, 2014.

[369] O. E. Philippova and E. V. Korchagina, "Chitosan and its hydrophobic derivatives: Preparation and aggregation in dilute aqueous solutions," Polymer Science Series A, vol. 54, no. 7, pp. 552-572, 2012.

[370] E. Szymańska and K. Winnicka, "Stability of chitosan-a challenge for pharmaceutical and biomedical applications.," Marine drugs, vol. 13, no. 4, pp. 181946, 2015.

[371] A. Domard, M. Rinaudo, and C. Terrassin, "New method for the quaternization of chitosan," International Journal of Biological Macromolecules, vol. 8, pp. 105-107, apr 1986. 
[372] R. C. Rowe, P. J. Sheskey, S. C. Owen, and American Pharmacists Association., Handbook of pharmaceutical excipients. Pharmaceutical Press, 2009.

[373] A. K. Nayak and P. P. Panigrahi, "Solubility Enhancement of Etoricoxib by Cosolvency Approach," ISRN Physical Chemistry, vol. 2012, pp. 1-5, 2012.

[374] R. G. Strickley, "Solubilizing Excipients in Oral and Injectable Formulations," Pharmaceutical Research, vol. 21, no. 2, pp. 201-230, 2004.

[375] J. Key, D. Dhawan, C. L. Cooper, D. W. Knapp, K. Kim, I. C. Kwon, K. Choi, K. Park, P. Decuzzi, and J. F. Leary, "Multicomponent, peptide-targeted glycol chitosan nanoparticles containing ferrimagnetic iron oxide nanocubes for bladder cancer multimodal imaging.," International journal of nanomedicine, vol. 11, pp. 4141-55, 2016.

[376] J. H. Park, S. Kwon, J.-O. Nam, R.-W. Park, H. Chung, S. B. Seo, I.-S. Kim, I. C. Kwon, and S. Y. Jeong, "Self-assembled nanoparticles based on glycol chitosan bearing 5beta-cholanic acid for RGD peptide delivery.," Journal of controlled release : official journal of the Controlled Release Society, vol. 95, no. 3, pp. 579-88, 2004.

[377] R. F. Domingos, M. a. Baalousha, Y. Ju-nam, M. M. Reid, N. Tufenkji, J. R. Lead, G. G. Leppard, and K. J. Wilkinson, "Characterizing Manufactured Nanoparticles in the Environment : Multimethod Determination of Particle Sizes Characterizing Manufactured Nanoparticles in the Environment : Multimethod Determination of Particle Sizes," Environmental Science \& Technology, pp. 7277-7284, 2009.

[378] L. Di, "Strategic approaches to optimizing peptide ADME properties.," The AAPS journal, vol. 17, no. 1, pp. 134-43, 2015.

[379] A. des Rieux, V. Fievez, M. Garinot, Y.-J. Schneider, and V. Préat, "Nanoparticles as potential oral delivery systems of proteins and vaccines: A mechanistic approach," Journal of Controlled Release, vol. 116, no. 1, pp. 1-27, 2006.

[380] M. Ginsburg and M. W. Smith, "The fate of oxytocin in male and female rats.," British journal of pharmacology and chemotherapy, vol. 14, pp. 327-333, 1959. 
[381] M. Fabian, M. L. Forsling, J. J. Jones, and J. S. Pryor, "The clearance and antidiuretic potency of neurohypophysial hormones in man, and their plasma binding and stability," The Journal of Physiology, vol. 204, pp. 653-668, oct 1969.

[382] A. Szeto, P. M. McCabe, D. A. Nation, B. A. Tabak, M. A. Rossetti, M. E. McCullough, N. Schneiderman, and A. J. Mendez, "Evaluation of enzyme immunoassay and radioimmunoassay methods for the measurement of plasma oxytocin.," Psychosomatic medicine, vol. 73, no. 5, pp. 393-400, 2011.

[383] K. H. Min, K. Park, Y. S. Kim, S. M. Bae, S. Lee, H. G. Jo, R. W. Park, I. S. Kim, S. Y. Jeong, K. Kim, and I. C. Kwon, "Hydrophobically modified glycol chitosan nanoparticles-encapsulated camptothecin enhance the drug stability and tumor targeting in cancer therapy," Journal of Controlled Release, vol. 127, no. 3, pp. 208-218, 2008.

[384] L. M. Ensign, R. Cone, and J. Hanes, “Oral drug delivery with polymeric nanoparticles: The gastrointestinal mucus barriers," Advanced Drug Delivery Reviews, vol. 64, no. 6, pp. 557-570, 2012.

[385] A. Fjellestad-Paulsen, C. Söderberg-Ahlm, and S. Lundin, "Metabolism of vasopressin, oxytocin, and their analogues in the human gastrointestinal tract.," Peptides, vol. 16, no. 6, pp. 1141-7, 1995.

[386] B. Matuszewska, G. Liversidge, F. Ryan, J. Dent, and P. Smith, "In vitro study of intestinal absorption and metabolism of 8-1-arginine vasopressin and its analogues," International Journal of Pharmaceutics, vol. 46, no. 1-2, pp. 111-120, 1988.

[387] Y. Zhang, W. Wei, P. Lv, L. Wang, and G. Ma, "Preparation and evaluation of alginate-chitosan microspheres for oral delivery of insulin," European Journal of Pharmaceutics and Biopharmaceutics, vol. 77, no. 1, pp. 11-19, 2011.

[388] C. J. Thompson, L. Tetley, I. F. Uchegbu, and W. P. Cheng, “The complexation between novel comb shaped amphiphilic polyallylamine and insulin: towards oral insulin delivery.," International journal of pharmaceutics, vol. 376, no. 12, pp. 46-55, 2009. 
[389] J. M. Sands and H. E. Layton, "The Physiology of Urinary Concentration: An Update," Seminars in Nephrology, vol. 29, no. 3, pp. 178-195, 2009.

[390] P. Lesley A. Stevens, M.D., Josef Coresh, M.D., Ph.D., Tom Greene and M. and Andrew S. Levey, “Assessing Kidney Function - Measured and Estimated Glomerular Filtration Rate," New England journal of medicine, pp. 2473-2483, 2006.

[391] I. Altun, N. D. Cinar, and M. K. Kasikci, "Self-reported quantity of daily water intake and urine output in healthy young," International Journal of Urological Nursing, vol. 6, no. 2, pp. 91-93, 2012.

[392] T. L. Pannabecker, W. H. Dantzler, H. E. Layton, and A. T. Layton, "Role of three-dimensional architecture in the urine concentrating mechanism of the rat renal inner medulla," AJP: Renal Physiology, vol. 295, no. 5, pp. F1271-F1285, 2008.

[393] S. Nielsen, B. L. Smith, E. I. Christensen, M. A. Knepper, and P. Agre, “CHIP28 water channels are localized in constitutively water-permeable segments of the nephron," Journal of Cell Biology, vol. 120, no. 2, pp. 371-383, 1993.

[394] X.-Y. Zhai, R. a. Fenton, A. Andreasen, J. S. Thomsen, and E. I. Christensen, "Aquaporin-1 is not expressed in descending thin limbs of short-loop nephrons.," Journal of the American Society of Nephrology : JASN, vol. 18, no. 11, pp. 2937-2944, 2007.

[395] F. Magni, C. Sarto, D. Ticozzi, M. Soldi, N. Bosso, P. Mocarelli, and M. G. Kienle, "Proteomic knowledge of human aquaporins," Proteomics, vol. 6, no. 20 , pp. 5637-5649, 2006.

[396] R. E. Day, P. Kitchen, D. S. Owen, C. Bland, L. Marshall, A. C. Conner, R. M. Bill, and M. T. Conner, "Human aquaporins: Regulators of transcellular water flow," Biochimica et Biophysica Acta - General Subjects, vol. 1840, no. 5, pp. 1492-1506, 2014. 
[397] A. B. Maunsbach, D. Marples, E. Chin, G. Ning, C. Bondy, P. Agre, and S. Nielsen, "Aquaporin-1 water channel expression in human kidney.," Journal of the American Society of Nephrology : JASN, vol. 8, no. 1, pp. 1-14, 1997.

[398] S. Nielsen, "Renal aquaporins : an overview," BJU International, vol. 90, pp. 27, 2002.

[399] M. Imai and J. P. Kokko, "Sodium Chloride , Urea, and Water Transport in the Thin Ascending Limb of Henle," The Journal of Clinical Investigation, vol. 53, no. February, pp. 393-402, 1974.

[400] N. Obermiiller, S. Kunchaparty, D. H. Ellison, and S. Bachmann, "Rapid Publication ansporter by Macula Densa and Thick Ascending Expression of the Na-K-2CI Limb Cells of Rat and Rabbit Nephron,” vol. 9, no. August, pp. 635640, 1996.

[401] J. M. Sands and M. A. Knepper, "Urea permeability of inner medullary collecting duct and papillary surface epithelium," Kidney International, vol. 29, p. 418, 1986.

[402] A. Jakes and S. Bhandari, "Investigating polyuria," BMJ: British Medical Journal, vol. 6772, no. December, pp. 1-5, 2013.

[403] Diabetes UK, “DIABETES PREVALENCE 2015." https://www.diabetes.org.uk/Professionals/Position-statementsreports/Statistics/diabetes-prevalence-2015/, 2015 [Date Accessed:10 October 2016].

[404] P. Van Kerrebroeck, P. Abrams, D. Chaikin, J. Donovan, D. Fonda, S. Jackson, P. Jennum, T. Johnson, G. R. Lose, A. Mattiasson, G. L. Robertson, J. Weiss, and International Continence Society, "The standardization of terminology in nocturia: report from the standardization subcommittee of the International Continence Society.," BJU international, vol. 90 Suppl 3, pp. 11-5, 2002.

[405] J. L. H. R. Bosch and J. P. Weiss, "The prevalence and causes of nocturia.," The Journal of urology, vol. 189, no. 1 Suppl, pp. S86-92, 2013. 
[406] F. M. Friedman and J. P. Weiss, "Desmopressin in the treatment of nocturia: clinical evidence and experience.," Therapeutic advances in urology, vol. 5, no. 6 , pp. 310-7, 2013.

[407] M. H. Ebell, T. Radke, and J. Gardner, "A systematic review of the efficacy and safety of desmopressin for nocturia in adults," Journal of Urology, vol. 192, no. 3, pp. 829-835, 2014.

[408] T. Nevéus, A. von Gontard, P. Hoebeke, K. Hjälmås, S. Bauer, W. Bower, T. M. Jørgensen, S. Rittig, J. V. Walle, C.-K. Yeung, and J. C. Djurhuus, "The standardization of terminology of lower urinary tract function in children and adolescents: report from the Standardisation Committee of the International Children's Continence Society.," The Journal of urology, vol. 176, no. 1, pp. 314-24, 2006.

[409] P. F. Austin, S. B. Bauer, W. Bower, J. Chase, I. Franco, P. Hoebeke, S. Rittig, J. Vande Walle, A. von Gontard, A. Wright, S. S. Yang, and T. Nevéus, "The standardization of terminology of lower urinary tract function in children and adolescents: update report from the Standardization Committee of the International Children's Continence Society.," The Journal of urology, vol. 191, no. 6, pp. 1863-1865.e13, 2014.

[410] A. von Gontard, K. Mauer-Mucke, J. Plück, W. Berner, and G. Lehmkuhl, "Clinical behavioral problems in day- and night-wetting children.," Pediatric nephrology (Berlin, Germany), vol. 13, pp. 662-7, oct 1999.

[411] W. L. Robson and A. K. Leung, "Secondary nocturnal enuresis.," Clinical pediatrics, vol. 39, no. 7, pp. 379-85, 2000.

[412] E. Bakker, M. van Sprundel, J. C. van der Auwera, J. D. van Gool, and J. J. Wyndaele, "Voiding habits and wetting in a population of 4,332 Belgian schoolchildren aged between 10 and 14 years.," Scandinavian journal of urology and nephrology, vol. 36, no. 5, pp. 354-62, 2002.

[413] O. Al-Omar, J. DiBianco, and C. Morley, "Nocturnal enuresis: A topic review and institution experience," Avicenna Journal of Medicine, vol. 4, no. 4, p. 77, 2014. 
[414] C. M. Glazener and J. H. Evans, "Desmopressin for nocturnal enuresis in children.," Cochrane database of systematic reviews (Online), no. 3, p. CD002112, 2002.

[415] J. M. Monda and D. A. Husmann, "Primary Nocturnal Enuresis: A Comparison Among Observation, Imipramine, Desmopressin Acetate and Bed-Wetting Alarm Systems," The Journal of Urology, vol. 154, no. 2, pp. 745-748, 1995.

[416] Johns Hopkins Univeristy, "Species Specific Information: Rat." http://web.jhu.edu/animalcare/procedures/rat.html, [Date Accessed: 10 October 2016].

[417] Rat and Mouse Club of America, "Rat Medical Cheat Sheet." http://www.rmca.org/Rescue/ratmedhelp.pdf, 2014 [Date Accessed:10 October 2016].

[418] C. L. Riphagen and Q. J. Pitrman, “on Blood Pressure, Heart Rate and Urine Output,” vol. 374, pp. 371-374, 1986.

[419] M. L. Forshing, M. Brimble, and R. J. Balment, "The influence of vasopressin on oxytocin-induced changes in urine flow in the male rat," European Journal of Endocrinology, vol. 100, no. 2, pp. 216-220, 1982.

[420] M. L. Kauker, J. T. Crofton, L. Share, and A. Nasjletti, "Role of vasopressin in regulation of renal kinin excretion in Long-Evans and diabetes insipidus rats," Journal of Clinical Investigation, vol. 73, no. 3, pp. 824-831, 1984.

[421] P. Bardoux, D. G. Bichet, H. Martin, Y. Gallois, M. Marre, M.-F. Arthus, M. Lonergan, N. Ruel, N. Bouby, and L. Bankir, "Vasopressin increases urinary albumin excretion in rats and humans : involvement of V 2 receptors and the renin - angiotensin system," Nephrology Dialysis Transplantation, vol. 18, pp. 497-506, 2003.

[422] S. C. Gad, Handbook of pharmaceutical biotechnology. Wiley-Interscience, 2007. 
[423] S. Lundin, H. G. Folkesson, S. G. Pierzynovski, and H. I. Bengtsson, "Gastrointestinal absorption and plasma clearance rates of [D-Arg8] vasopressin analogues in the rat," Peptides, vol. 15, no. 5, pp. 809-14, 1994.

[424] T. Søeborg, C. H. Rasmussen, E. Mosekilde, and M. Colding-Jørgensen, "Bioavailability and variability of biphasic insulin mixtures," European Journal of Pharmaceutical Sciences, vol. 46, no. 4, pp. 198-208, 2012.

[425] N. A. Peppas, J. Z. Hilt, and J. B. Thomas, Nanotechnology in therapeutics : current technology and applications. Horizon Bioscience, 2007.

[426] K. W. Joo, U. S. Jeon, G. H. Kim, J. Park, Y. K. Oh, Y. S. Kim, C. Ahn, S. Kim, S. Y. Kim, J. S. Lee, and J. S. Han, "Antidiuretic action of oxytocin is associated with increased urinary excretion of aquaporin-2," Nephrology Dialysis Transplantation, vol. 19, no. 10, pp. 2480-2486, 2004.

[427] R. Liu, Water-insoluble drug formulation. CRC Press, 2nd ed. ed., 2008.

[428] B. Chini and M. Manning, "Agonist selectivity in the oxytocin/vasopressin receptor family: new insights and challenges.," Biochemical Society transactions, vol. 35, no. 4, pp. 737-741, 2007.

[429] M. Manning, S. Stoev, B. Chini, T. Durroux, B. Mouillac, and G. Guillon, "Peptide and non-peptide agonists and antagonists for the vasopressin and oxytocin V1a, V1b, V2 and OT receptors: research tools and potential therapeutic agents," Progress in Brain Research, vol. 170, no. 08, pp. 473-512, 2008.

[430] J. Wang, V. Yadav, A. L. Smart, S. Tajiri, and A. W. Basit, "Toward oral delivery of biopharmaceuticals: An assessment of the gastrointestinal stability of 17 peptide drugs," Molecular Pharmaceutics, vol. 12, no. 3, pp. 966-973, 2015.

[431] J. Nestor Jr., "The Medicinal Chemistry of Peptides," Current Medicinal Chemistry, vol. 16, no. 33, pp. 4399-4418, 2009.

[432] F. A. Fisusi, A. Siew, K. W. Chooi, O. Okubanjo, N. Garrett, K. Lalatsa, D. Serrano, I. Summers, J. Moger, P. Stapleton, R. Satchi-Fainaro, A. G. Schätzlein, 
and I. F. Uchegbu, "Lomustine Nanoparticles Enable Both Bone Marrow Sparing and High Brain Drug Levels - A Strategy for Brain Cancer Treatments," Pharmaceutical Research, vol. 33, no. 5, pp. 1289-1303, 2016.

[433] M. B. Pastrian, F. Guzmán, J. Garona, M. Pifano, G. V. Ripoll, O. Cascone, G. N. Ciccia, F. Albericio, D. E. Gómez, D. F. Alonso, and N. B. Iannucci, "Structure-activity relationship of 1-desamino-8-D-arginine vasopressin as an antiproliferative agent on human vasopressin V2 receptor-expressing cancer cells.," Molecular medicine reports, vol. 9, pp. 2568-72, jun 2014. 